Portland State University

PDXScholar

Fall 12-3-2019

\title{
Optimizing On-Street Parking Supply with Analytical Models
}

Chawalit Tipagornwong

Portland State University

Follow this and additional works at: https://pdxscholar.library.pdx.edu/open_access_etds

Part of the Transportation Engineering Commons

Let us know how access to this document benefits you.

Recommended Citation

Tipagornwong, Chawalit, "Optimizing On-Street Parking Supply with Analytical Models" (2019).

Dissertations and Theses. Paper 5353.

https://doi.org/10.15760/etd.7226

This Dissertation is brought to you for free and open access. It has been accepted for inclusion in Dissertations and Theses by an authorized administrator of PDXScholar. Please contact us if we can make this document more accessible: pdxscholar@pdx.edu. 
Optimizing On-Street Parking Supply with Analytical Models

by

Chawalit Tipagornwong

A dissertation submitted in partial fulfillment of the requirements for the degree of

\author{
Doctor of Philosophy \\ in
}

Civil and Environmental Engineering

Dissertation Committee:

Miguel A. Figliozzi, Chair

Christopher M. Monsere

Kelly J. Clifton

Feng Liu

Portland State University

2019 


\begin{abstract}
Commercial vehicles are crucial for the urban economy, but they generate negative impacts as well. This leads to restrictions such as a reduction in the number of loading zones. The result is that commercial drivers spend a significant amount of time searching for available loading zones. To avoid searching for parking, some commercial drivers double park, which causes traffic congestion. Therefore, the number of loading zones should be set properly. Since a curb is allocated between both loading zones and parking spaces, both parking types have to be considered. Many on-street parking management strategies have been implemented, including pricing meter rates and allocating curb length to both parking types.
\end{abstract}

Analytical models are proposed to manage an on-street parking system, including loading zones for commercial vehicles and parking spaces for passenger cars. The models give optimal solutions to the system, including curb length allocation and meter rates for both parking types. The models' objectives are to maximize revenue and to maximize social optimum which equals revenue minus driver costs and externality costs. The costs are parking search cost, traffic delay cost due to parking maneuver in and out of parking spots, and traffic delay cost due to double parking.

The optimal results from case studies demonstrate how the arrival rate of parking vehicles affects the meter rate. If the arrival rate is low, compared with parking supply, all vehicles will park on the street. The meter rate depends on the arrival rate. As the arrival rate increases, the parking search cost will increase. To keep the on-street option cheaper than the other parking option, the meter rate has to decrease. However, if the arrival rate is 
high, compared with parking supply, some drivers will park on-street while others will choose the other parking option. The meter rate usually does not depend on the arrival rate.

The optimal results from case studies demonstrate how the arrival rates of parking vehicles affect curb length allocation. If the arrival rates are low, much curb length will be allocated to a parking type of which meter rate is the most related to the allocation. However, if the arrival rates are high, much curb length will be allocated to a parking type of which revenue demand is the most related to the allocation. In practice, though, the meter rate usually does not depend on the allocation. Compared with the revenue model, the social optimum model allocates higher curb length to a parking type of which counterpart causes more externalities. For example, a commercial vehicle double parking causes a lot of traffic delay costs. The SOC model allocates higher curb length to loading zones than the revenue model does.

A commercial vehicle often double parks in a downtown area where there are a lot of customers and intersection intensity is high. A double-parking vehicle causes traffic congestion and the traffic queue might spill back to an intersection upstream of the vehicle. A traffic signal at the upstream intersection affects traffic delays caused by the doubleparking event because the traffic signal changes distribution of traffic flow rates. If the vehicle starts to double park when the flow rate is saturation flow rate, the traffic delays will be the highest. A double-parking vehicle also delays parking passenger cars since passenger cars travels on roads while searching for available on-street parking spaces. 


\section{Acknowledgements}

The research in this dissertation was supported by the Royal Thai Scholarship Program and the Maseeh College of Engineering and Computer Science at Portland State University. This research was conducted under the guidance of my advisor Professor Dr. Miguel Figliozzi. He was very supportive and patient with me.

I appreciate all students in the Transportation Technology and People (TTP) lab and the Students in Transportation Engineering and Planning (STEP) group for their friendship and support, especially Steven Gehrke and Kristina Currans for providing me with a lot of useful information. I feel thankful to Aphisit Phoowarawutthipanich, Ekapon Akarapanitkorn, Tom Downing, James L. Bunch, Sandra M. Bunch, Duane T. Decker, Christopher D. Starr, Laura Bieber, and Kitti Manokhoon for great motivation. Krongkaew Tipagornwong always took care of and encouraged me while I was studying in the U.S.

I would like to thank my father, my mother, my brother, and my sister for consistent support. My father explored the U.S. for our family. I am deeply thankful for my mother who always encouraged me, gave me valuable advice, and trusted in me for the whole of my life. 


\section{Table of Contents}

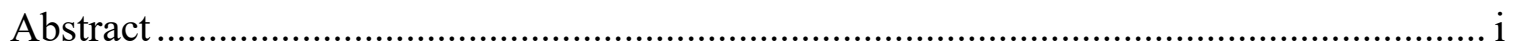

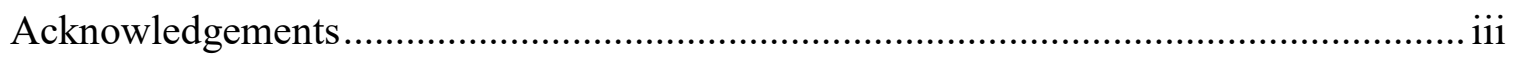

List of Tables ................................................................................................... viii

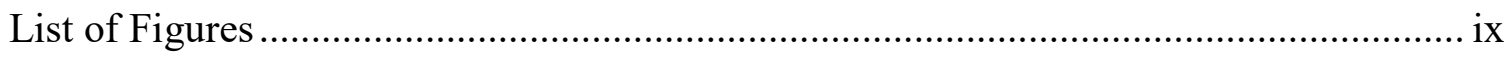

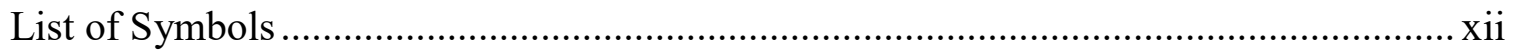

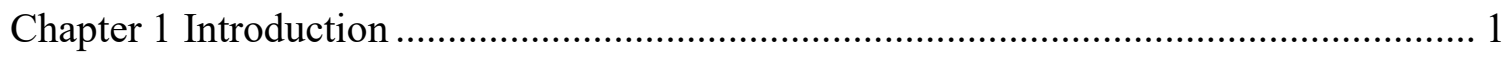

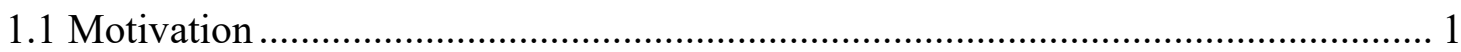

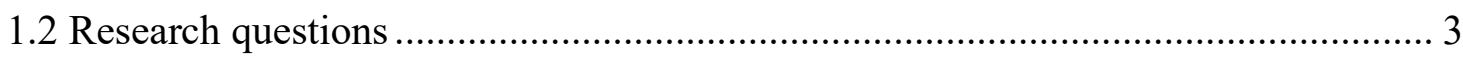

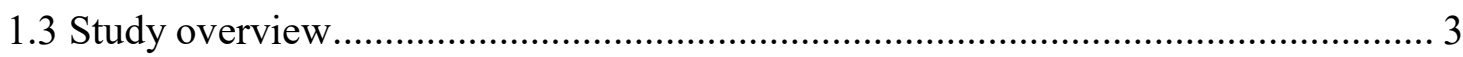

Chapter 2 Literature Review .............................................................................. 5

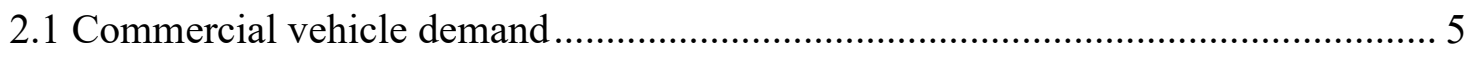

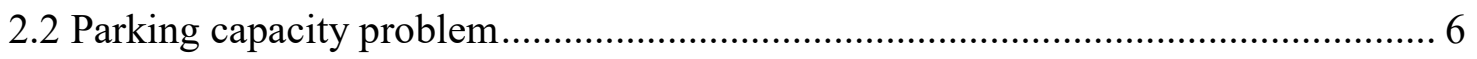

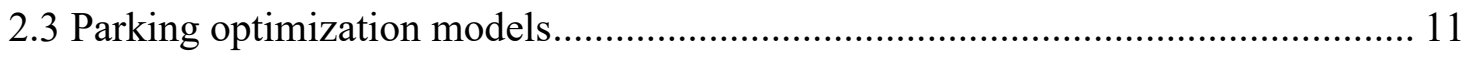

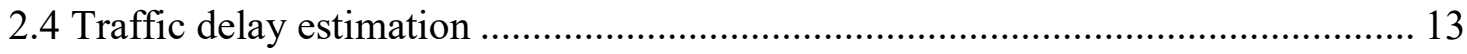

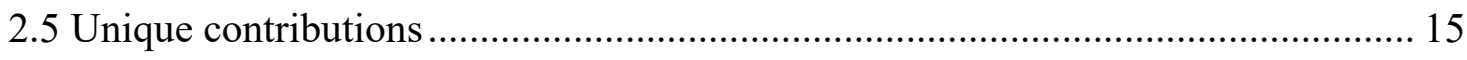

Chapter 3 Traffic Delays of On-Street Parking System............................................... 19

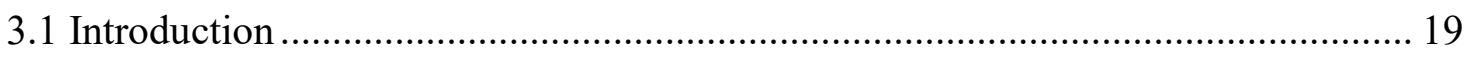

3.2 Fundamental flow density diagram ........................................................... 19

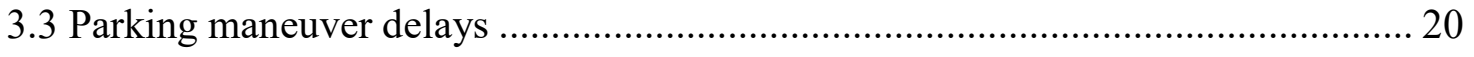




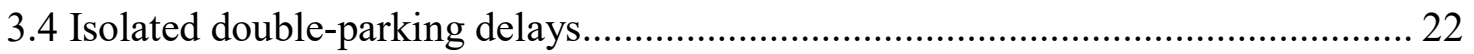

3.5 Corridor double parking delays ........................................................................ 25

3.6 Simulation for estimating double-parking delays ............................................... 34

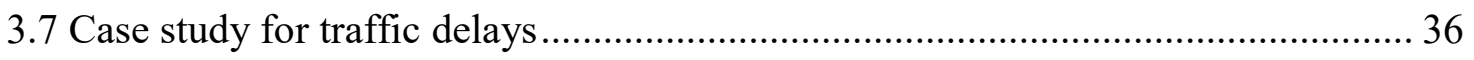

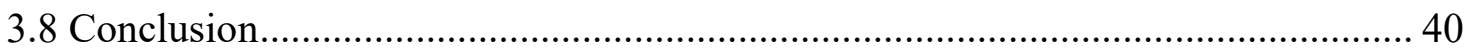

Chapter 4 Overview of On-Street Parking Models......................................................... 41

4.1 Description of on-street parking system.............................................................. 41

4.2 Objective function and decision variable ............................................................. 43

4.3 Estimation of costs associated with parking …………...................................... 43

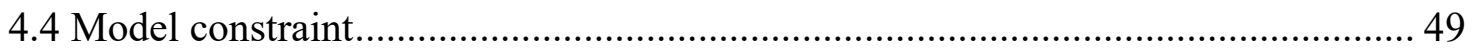

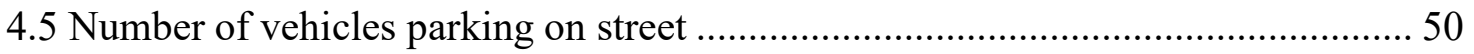

4.6 Scenarios for developing parking models ....................................................... 55

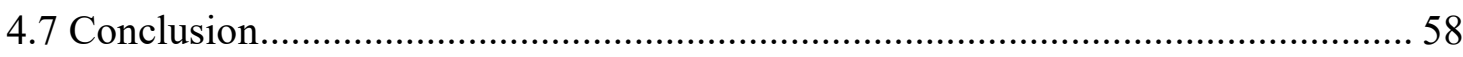

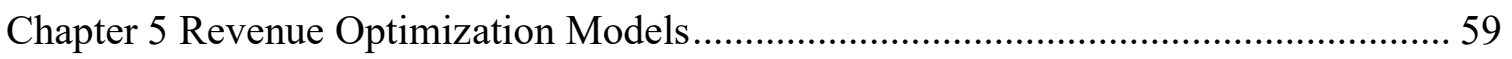

5.1 Definition of revenue optimization models.......................................................... 59

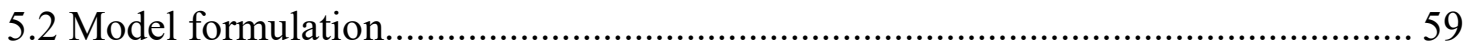

5.3 Revenue model for Scenario 1: All vehicles parking on street................................ 60

5.4 Revenue model for Scenario 2: Some passenger cars park off street ...................... 63

5.5 Revenue model for Scenario 3: Some commercial vehicles double park ............... 66

5.6 Revenue model for Scenario 4: All parking options are selected ........................... 69 
5.7 Solution summary and interpretation

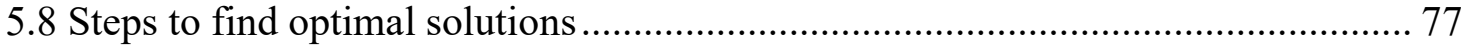

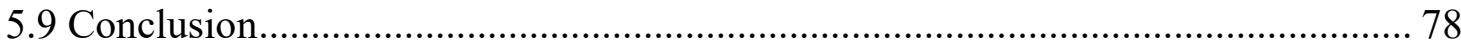

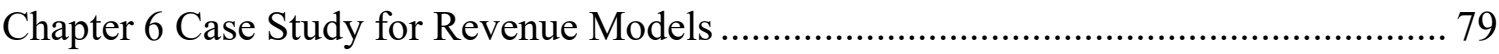

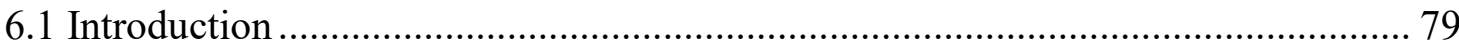

6.2 Optimal solutions for passenger car parking equivalent ......................................... 81

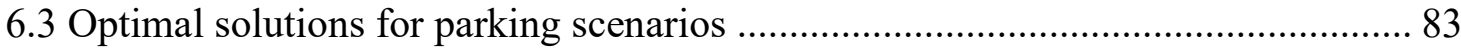

6.4 Impact of exponents of the parking search time function ..................................... 94

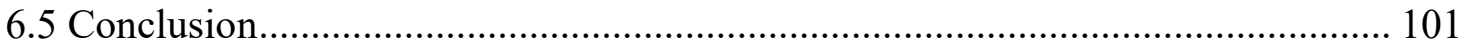

Chapter 7 Social Optimum Optimization Models ......................................................... 103

7.1 Definition of social optimum optimization models.................................................. 103

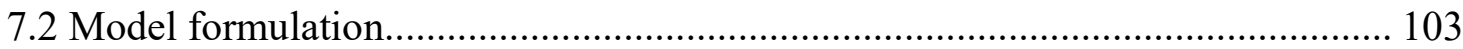

7.3 SOC model for Scenario 1: All vehicles parking on street ................................. 104

7.4 SOC model for Scenario 2: Some passenger cars park off street.......................... 106

7.5 SOC model for Scenario 3: Some commercial vehicles double park .................... 109

7.6 SOC model for Scenario 4: All parking options are selected ................................ 112

7.7 Solution summary and interpretation ............................................................... 116

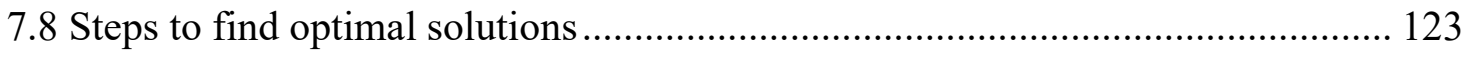

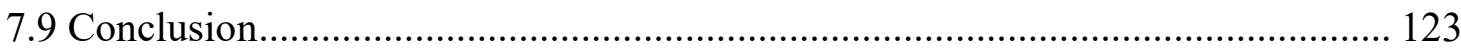


Chapter 8 Case Study for Social Optimum Models

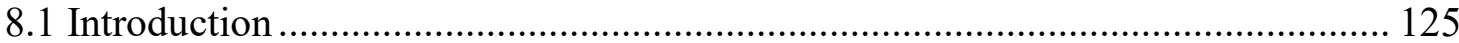

8.2 Optimal solutions for passenger car parking equivalent .................................. 126

8.3 Optimal solutions for parking scenarios ................................................... 127

8.4 Impact of exponents of the parking search time function ............................... 139

8.5 Compare the revenue models with the social optimum models .......................... 145

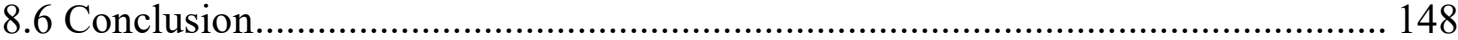

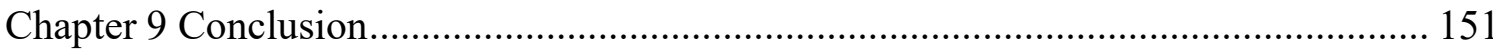

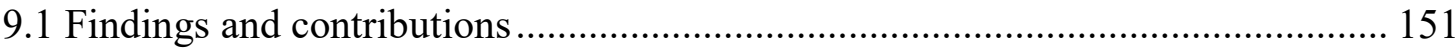

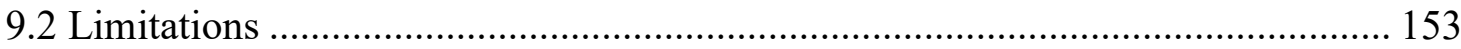

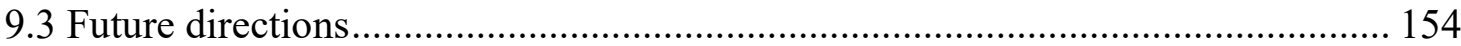

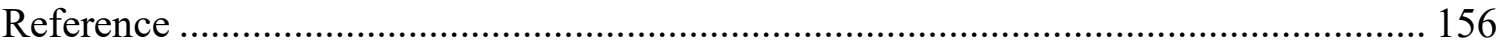

Appendix A: Full derivation of parking supply allocation and pricing models ............ 169

Appendix B: R code for corridor traffic delay simulation ......................................... 221 


\section{List of Tables}

Table 2.1 Commercial parking optimization models.................................16

Table 2.2 Passenger parking management models...............................17

Table 3.1 Base data for traffic delay estimation due to a double-parking event...........36

Table 4.1 Parking management strategy and scenarios for parking models ..............56

Table 5.1 Solutions to revenue models for 4 scenarios............................ 74

Table 5.2 Interpretation of optimal loading zone meter rate for revenue models..........76

Table 5.3 Interpretation of optimal parking space meter rate for revenue models........76

Table 5.4 Interpretation of optimal curb length allocation for revenue models...........77

Table 6.1 Base data for case studies........................................ 80

Table 6.2 Arrival rates of parking vehicle for 4 scenarios............................ 81

Table 6.3 Solutions of revenue models: vehicle equivalent parking on street............82

Table 6.4 Solutions of revenue models: vehicle equivalent parking on- and off-street....82

Table 6.5 Optimal solutions for the revenue models $\left(\gamma_{c}=\gamma_{p}=2\right) \ldots \ldots \ldots \ldots \ldots \ldots \ldots . . . \ldots 3$

Table 6.6 Estimates of parking search cost.....................................95

Table 7.1 Solutions to social-optimum models for 4 scenarios.....................117

Table 7.2 Interpretation of optimal loading zone meter rate for SOC models...........121

Table 7.3 Interpretation of optimal parking space meter rate for SOC models............122

Table 7.4 Interpretation of optimal curb length allocation for SOC models............122

Table 8.1 Solutions of SOC models: vehicle equivalent parking on street..............126

Table 8.2 Solutions of SOC models: vehicle equivalent parking on- and off-street......127

Table 8.3 Optimal solutions for the social optimum models $\left(\gamma_{c}=\gamma_{p}=2\right) \ldots \ldots \ldots \ldots \ldots 138$

Table 8.4 Comparison of optimal solutions of revenue and SOC models................148 viii 


\section{List of Figures}

Figure 1.1 Conceptual framework for this study..................................4

Figure 2.1 Hourly on-street commercial parking demand from literature................7

Figure 2.2 Dissipation rates nearby an intersection downstream of blockage...............15

Figure 3.1 Fundamental diagram for a 2-lane roadway............................20

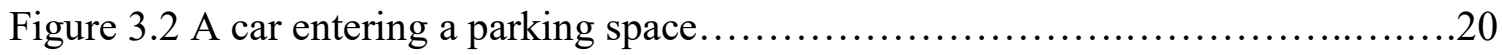

Figure 3.3 Deterministic queuing diagram for parking maneuver.....................21

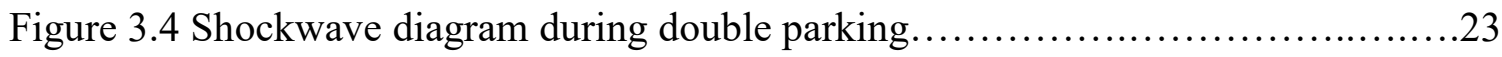

Figure 3.5 Shockwave diagram after double parking ends..........................24

Figure 3.6 Deterministic queuing diagram of an isolated double parking................25

Figure 3.7 Shockwave diagram of a double-parking event in a corridor................27

Figure 3.8 Queuing diagram of the entry intersection of a corridor...................28

Figure 3.9 Queue diagram for corridor double parking..............................30

Figure 3.10 Deterministic queuing diagram from the simulation..................... 35

Figure 3.11 Traffic parking-maneuver delay cost of a passenger car....................38

Figure 3.12 Traffic parking-maneuver delay cost of a commercial vehicle...............38

Figure 3.13 Corridor double-parking traffic delay cost........................... 39

Figure 3.14 Double-parking traffic delays per car per one double-parking event..........40

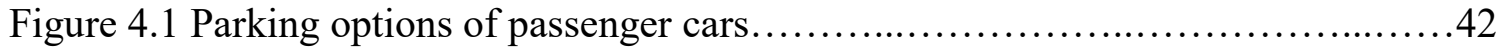

Figure 4.2 Parking options of commercial vehicles...............................43

Figure 4.3 Joint effect on car breakeven demand..................................54

Figure 4.4 Scenario 1: All vehicles parking on street............................56

Figure 4.5 Scenario 2: Some passenger cars parking off street......................57 
Figure 4.6 Scenario 3: Some commercial vehicles double parking...................57

Figure 4.7 Scenario 4: All parking options are chosen..............................58

Figure 6.1 Optimal meter rates of scenario-1 revenue model.......................84

Figure 6.2 On-street scenario-1 parking revenue................................84

Figure 6.3 Optimal meter rates of scenario-2 revenue model.......................86

Figure 6.4 Revenue demand of scenario-2 revenue model.........................86

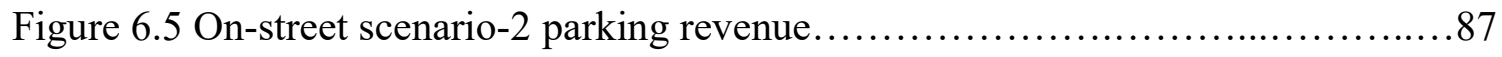

Figure 6.6 Optimal meter rates of scenario-3 revenue model.......................88

Figure 6.7 Revenue demand of scenario-3 revenue model.........................89

Figure 6.8 On-street scenario-3 parking revenue............................. 90

Figure 6.9 Revenue demand of scenario-4 revenue model.........................91

Figure 6.10 On-street scenario-4 parking revenue.............................92

Figure 6.11 Impact of exponents on cost components of on-street parking.............95

Figure 6.12 Impact of exponents on the revenue for scenario $1 \ldots \ldots \ldots \ldots \ldots \ldots \ldots \ldots . . .67$

Figure 6.13 Impact of exponents on the revenue for scenario $2 \ldots \ldots \ldots \ldots \ldots \ldots \ldots \ldots 9$

Figure 6.14 Impact of exponents on the revenue for scenario $3 \ldots \ldots \ldots \ldots \ldots \ldots \ldots \ldots . . . \ldots 9$

Figure 6.15 Optimal rates for scenario-4 revenue models with different exponents......100

Figure 6.16 Impact of exponents on the revenue for scenario $4 \ldots \ldots \ldots \ldots \ldots \ldots \ldots \ldots \ldots$

Figure 8.1 Optimal meter rates of scenario-1 social optimum model.................128

Figure 8.2 On-street scenario-1 social optimum................................ 129

Figure 8.3 Optimal meter rates of scenario-2 social optimum model.................130

Figure 8.4 Revenue demand of scenario-2 social optimum model....................131

Figure 8.5 On-street scenario-2 social optimum................................ 132 
Figure 8.6 Optimal meter rates of scenario-3 social optimum model.

Figure 8.7 Revenue demand of scenario-3 social optimum model......................134

Figure 8.8 On-street scenario-3 social optimum.................................134

Figure 8.9 Optimal meter rates of scenario-4 social optimum model..................136

Figure 8.10 Revenue demand of scenario-4 social optimum model..................136

Figure 8.11 On-street scenario-4 social optimum..............................137

Figure 8.12 Impact of exponents on the scenario-1 social optimum.....................141

Figure 8.13 Impact of exponents on the scenario-2 social optimum...................142

Figure 8.14 Impact of exponents on the scenario-3 social optimum..................143

Figure 8.15 Optimal rates for scenario-4 SOC models from different exponents.........144

Figure 8.16 Impact of exponents on the social optimum for scenario $4 \ldots \ldots \ldots \ldots \ldots \ldots \ldots . \ldots 145$

Figure 8.17 Difference between $c_{p o}^{*}$ of revenue and social optimum models.............146

Figure 8.18 Difference between $\boldsymbol{c}_{\boldsymbol{c o}}^{*}$ of revenue and social optimum models..............147 


\section{List of Symbols}

\begin{tabular}{|c|c|c|}
\hline Notation & Definition & Unit \\
\hline$C_{l}$ & Traffic signal cycle length & Seconds \\
\hline$c_{c o}$ & Meter rate of on-street loading zones & Dollars per hour \\
\hline$c_{c t}$ & Value of time of a commercial vehicle & Dollars per hour \\
\hline$c_{d b}$ & Traffic delay cost per double-parking event & Dollars per event \\
\hline$c_{d c}$ & $\begin{array}{l}\text { Traffic delay cost due to a commercial vehicle } \\
\text { entering and exiting a loading zone }\end{array}$ & Dollars per vehicle \\
\hline$c_{d p}$ & $\begin{array}{l}\text { Traffic delay cost due to a passenger car } \\
\text { entering and exiting a parking space }\end{array}$ & Dollars per vehicle \\
\hline$c_{p o}$ & Meter rate of on-street parking spaces & Dollars per hour \\
\hline$c_{p f}$ & Meter rate of off-street parking lots or garages & Dollars per hour \\
\hline$c_{p f f}$ & Off-street passenger car parking fixed cost & Dollars per vehicle \\
\hline$c_{p t}$ & Value of time of a passenger car & Dollars per hour \\
\hline$c_{v}$ & Double-parking fines & $\$$ /ticket \\
\hline$d$ & Traffic delays per one double parking event & Vehicle-hours \\
\hline$g$ & Effective green time at intersections & Seconds \\
\hline$f_{d b}$ & $\begin{array}{l}\text { Traffic delays to a passenger car due to } \\
\text { double-parking event }\end{array}$ & Hour/event/vehicle \\
\hline $\mathrm{J}$ & $\begin{array}{l}\text { Joint impact of loading zone and parking space } \\
\text { utilization on delays of parking cars }\end{array}$ & \\
\hline$l_{b}$ & Available curb length per block & Feet \\
\hline$l_{c}$ & Unit length of a commercial vehicle loading zone & Feet \\
\hline$l_{p}$ & Unit length of a passenger car parking space & Feet \\
\hline NJ & None of the joint impact & \\
\hline$n_{a}$ & Number of equivalent parking spaces & \\
\hline$n_{c}$ & Number of commercial parking spaces & \\
\hline$n_{e}$ & Number of parking enforcement officers & \\
\hline$n_{p}$ & Number of on-street passenger car parking spaces & \\
\hline$Q_{\max }$ & Maximum queue length & Vehicles \\
\hline$q_{A}$ & Traffic volume of a corridor & Vehicles per hour \\
\hline$q_{B}$ & Effective flow rate at a double-parking location & Vehicles per hour \\
\hline$q_{C}$ & Roadway capacity without blocking & Vehicles per hour \\
\hline$q_{o}$ & Zero flow rate & Vehicles per hour \\
\hline$q_{s}$ & Saturation flow rate & Vehicles per hour \\
\hline
\end{tabular}




\begin{tabular}{|c|c|c|}
\hline Notation & Definition & Unit \\
\hline R1 & On-street parking revenue for scenario 1 & \\
\hline R2 & On-street parking revenue for scenario 2 & \\
\hline R3 & On-street parking revenue for scenario 3 & \\
\hline R4 & On-street parking revenue for scenario 4 & \\
\hline $\mathrm{SOC}$ & On-street parking social optimum & \\
\hline S1 & On-street parking social optimum for scenario 1 & \\
\hline $\mathrm{S} 2$ & On-street parking social optimum for scenario 2 & \\
\hline S3 & On-street parking social optimum for scenario 3 & \\
\hline $\mathrm{S} 4$ & On-street parking social optimum for scenario 4 & \\
\hline$t_{s}$ & Average on-street parking search time & Hours per car \\
\hline$y_{\text {rev }}$ & $\begin{array}{l}\text { Formula for calculating revenue of on-street parking } \\
\text { system }\end{array}$ & \\
\hline$y_{\text {soc }}$ & $\begin{array}{l}\text { Formula for calculating social optimum of on-street } \\
\text { parking system }\end{array}$ & \\
\hline$\alpha_{w c}$ & Waiting time commercial vehicle coefficient & Hours per vehicle \\
\hline$\alpha_{w p}$ & Waiting time passenger car coefficient & Hours per vehicle \\
\hline$\gamma_{c}$ & Waiting time commercial vehicle coefficient & - \\
\hline$\gamma_{p}$ & Waiting time passenger car coefficient & - \\
\hline$\vartheta$ & $\begin{array}{l}\text { Ratio of walking time to parking search time of } \\
\text { a passenger car }\end{array}$ & \\
\hline$\lambda_{a}$ & Aggregated arrival rate of equivalent passenger cars & Vehicles per hour \\
\hline$\lambda_{a}^{*}$ & Aggregated equivalent car breakeven demand & Vehicles per hour \\
\hline$\lambda_{c}$ & Arrival rate of commercial vehicles & Vehicles per hour \\
\hline$\lambda_{c r}$ & Number of revenue commercial vehicles parking on street & Vehicles per hour \\
\hline$\lambda_{c}^{*}$ & $\begin{array}{l}\text { Commercial vehicle breakeven demand (where } \\
\text { on-street parking cost equals double parking cost) }\end{array}$ & Vehicles per hour \\
\hline$\lambda_{p}$ & Arrival rate of passenger cars & Vehicles per hour \\
\hline$\lambda_{p r}$ & Number of revenue passenger cars parking on street & Vehicles per hour \\
\hline$\lambda_{p}^{*}$ & $\begin{array}{l}\text { Passenger car breakeven demand (where } \\
\text { on-street parking cost equals off-street parking cost) }\end{array}$ & Vehicles per hour \\
\hline$\mu_{c}$ & Service rate of a loading zone & Vehicles per hour \\
\hline$\mu_{p}$ & Service rate of a parking space & Vehicles per hour \\
\hline$\tau_{b}$ & Double parking duration & Hours \\
\hline$\tau_{b 1}$ & Duration of saturation flow in one traffic signal cycle & Hours \\
\hline$\tau_{d}$ & Queue dissipation duration & Hours \\
\hline$\tau_{e}$ & Parking enforcement cycle duration & Hours \\
\hline
\end{tabular}




\section{Chapter 1 Introduction}

\subsection{Motivation}

Goods deliveries and service visits are crucial for the urban economy. In an urban area, most establishments (e.g. retail stores, shops, grocery stores, and restaurants) rely on deliveries of merchandise and food ingredients. Service visits are also important because they support the regular activities of these establishments.

Although deliveries of goods and services are necessary to an urban economy, commercial vehicles generate negative impacts including traffic congestion, pollution, and traffic incidents, especially in a downtown area. These negative impacts lead to commercial vehicle restrictions in many urban areas. Typical restrictions include bans at certain times of day, noise and pollution restriction, vehicle size limits, and a reduction of loading zones.

The reduction in loading zones decreases parking availability for commercial vehicles. When loading zones are highly occupied, drivers have two options to respond: searching for available loading zones or double parking. Commercial vehicles double parking has been documented in many U.S. cities. For example, in midtown Manhattan (New York City), all delivery and service companies paid \$ 550 million for parking tickets in 2013 (Hawkins, 2013). In San Francisco, UPS, FedEx, and service providers received approximately 27,000 parking tickets in 2006 (Gordon, 2007). To offset the impact of parking fines, the delivery and service companies increase service fees, resulting in an increase in consumer expenses

Double parking not only affects consumers but also creates traffic delays. If a commercial vehicle driver decides to double park, one traffic lane will be blocked and road 
capacity will be reduced. The reduced road capacity may cause additional delays to other vehicles, especially if traffic volume is high. Traffic delays have to be estimated and responded to by the freight companies.

Providing the adequate number of loading zones can reduce the parking search time and the number of double-parking events. However, loading zones and passenger parking spaces share the same available curb length. Providing more loading zones leads to fewer parking spaces for passenger cars. This makes all the parking spaces occupied more frequently and car drivers spend more time to find available ones which increases parking cost of car drivers.

To manage both loading zones and parking spaces, a city planner has to implement multiple strategies, including allocating curb length to both parking types, pricing both parking types, operating a parking reservation system, or setting time windows for commercial vehicles. For an on-street parking system, a city planner might want to achieve multiple goals such as maximizing revenue from vehicles parking on street and minimizing externality costs of the system and maximizing social optimum, which equals the revenue minus costs associated with the on-street parking system.

This study focuses on two parking management strategies: setting meter rates and allocating curb length. Main objectives are to maximize the revenue and the social optimum. 


\subsection{Research questions}

The primary research question of this dissertation is:

- How to allocate curb length and set meter rates for on-street parking spaces where commercial vehicles are present in order to maximize revenue and/or to maximize social optimum?

The secondary research question is:

- How much may traffic delay cost be related to on-street parking of commercial vehicles?

The primary research question leads to the main objectives of this dissertation. The secondary research question leads to the externality cost of the on-street parking system, which is one of the objective value components.

\subsection{Study overview}

The focus of this study is to develop analytical models to maximize revenue (Chapter 5) and maximize the social optimum (Chapter 7) by optimizing decision variables. The decision variables are the meter rates and the curb length allocation. In the model, onstreet parking types are loading zones for commercial vehicles and parking spaces for passenger cars.

The model components; the parking search costs, the parking revenue, and the number of vehicles parking on street, are estimated using a parking availability model (Chapter 4). Traffic delays created by a double-parking event are estimated (Chapter 3). A conceptual framework of this study is shown in Figure 1.1. 


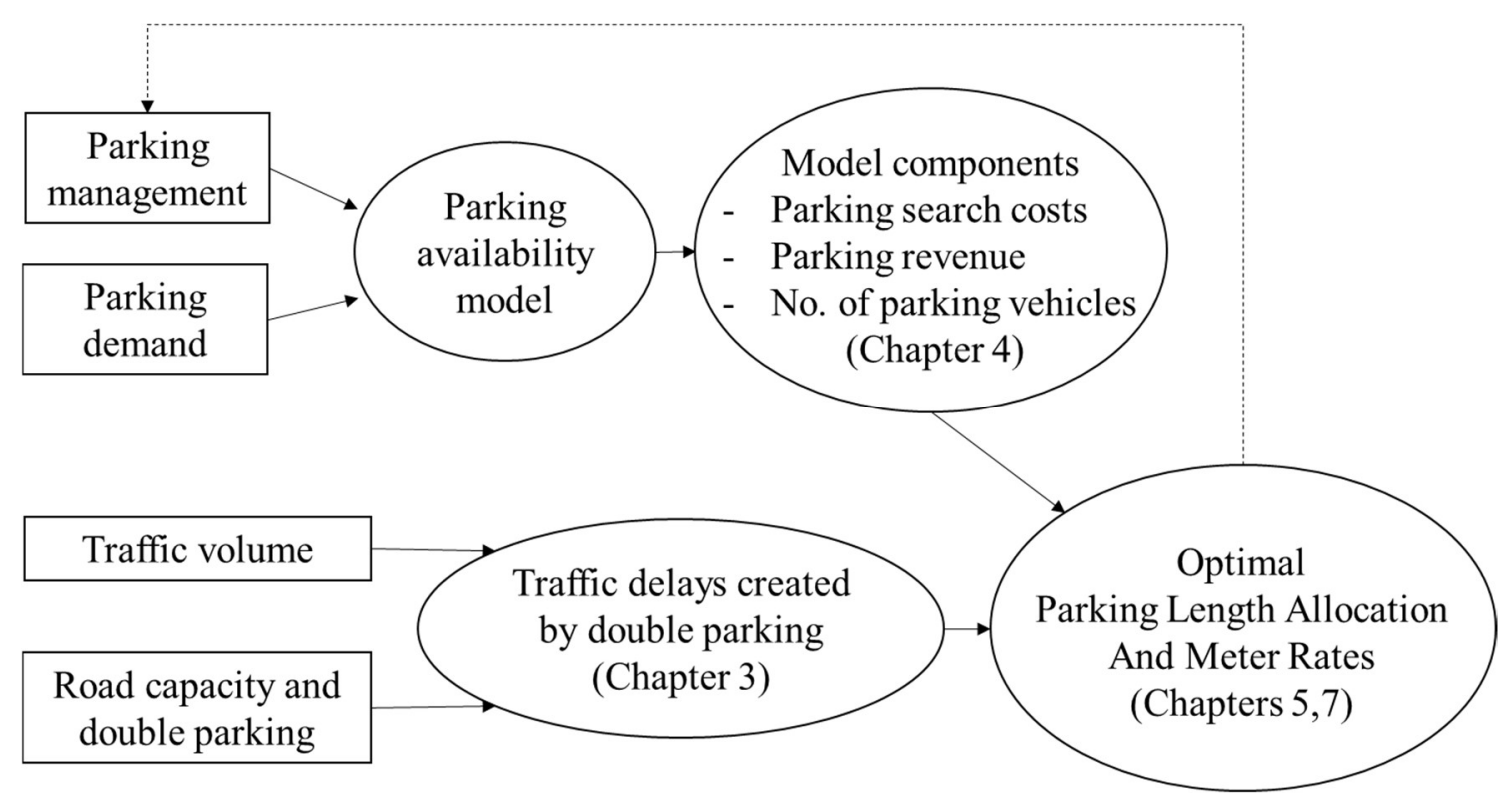

Figure 1.1 Conceptual framework for this study 


\section{Chapter 2 Literature Review}

This chapter includes characteristics of commercial vehicle demand and description of the inadequate parking problem in downtown areas. The chapter also reviews previous studies regarding parking optimization models and incident traffic delay estimation.

\subsection{Commercial vehicle demand}

This section includes service time of different commercial trip types and required parking lengths for different vehicle types.

\subsubsection{Commercial trip type and service time}

Urban commercial trips consist of goods delivery and service visit. Goods delivery can be classified into three groups: full truckload (FTL), less-than-truckload (LTL), and courier. Most of FTL and LTL deliveries are pallets or large parcels (Tozzi et al., 2012; Morris et al., 1999). Most of courier deliveries are small parcels and packages. Commercial vehicles for FTL are semitrailers and medium trucks while commercial vehicles for LTL and couriers can be box trucks and vans couriers (Murphy and Wood, 2010). In addition, the majority of service visits are equipment maintenance, cleaning, and food catering. Most vehicles for service visits are vans and cars (Cherrett, et al., 2012).

Service time of FTL is the longest while service time of courier is the shortest (Muñuzuri et al., 2012). Service time of some service visits is low while dwelling time of others is very high (Cherrett, et al., 2012). 


\subsubsection{Vehicle type and parking length}

The majority of commercial vehicles delivering freight in a downtown area are light (4-tire) commercial vehicles and medium (6+ tire) trucks (Fischer and Han, 2001). Compared with medium trucks, parking length for light vehicles are shorter. Light commercial vehicles also have more parking options than medium trucks do because some vans can park in passenger car parking spaces to unload goods via side doors (Dezi, et al., 2010).

Another commercial vehicle type is a freight tricycle. It is promising for delivering light goods including food and beverages, grocery products, office supplies, electronics, and pharmaceutical products (Maes and Vanelslander, 2012). In some downtown areas where traffic is congested, a freight tricycle can bypass traffic congestion if a city allows the utilization of bicycle lanes. Compared with other commercial vehicles, a tricycle needs the shortest parking length and, in some urban areas, tricycles can legally park on a sidewalk (Tipagornwong and Figliozzi, 2014).

\subsection{Parking capacity problem}

This section explains the cause of the parking capacity problem, how commercial vehicle drivers respond when loading zones are fully occupied, and how to assess the parking availability.

\subsubsection{Cause of parking capacity problem}

A parking inadequacy problem for commercial vehicles takes place when parking demand exceeds parking supply. Commercial vehicle parking demand is high especially in morning, as shown in Figure 2.1. Many businesses prefer to receive deliveries during peak hours because they have enough staff to accept deliveries (Holguin-Veras et al, 2005). In 
addition, on-street loading zones are sometimes occupied by non-commercial vehicles, for example, customers or commuters vehicles (Bomar, et al., 2009), passenger cars of shop owners (Muñuzuri, et al., 2012), sales representatives (Pivo, et al., 1997), and other nonfreight vehicles (Pivo, et al., 1997)

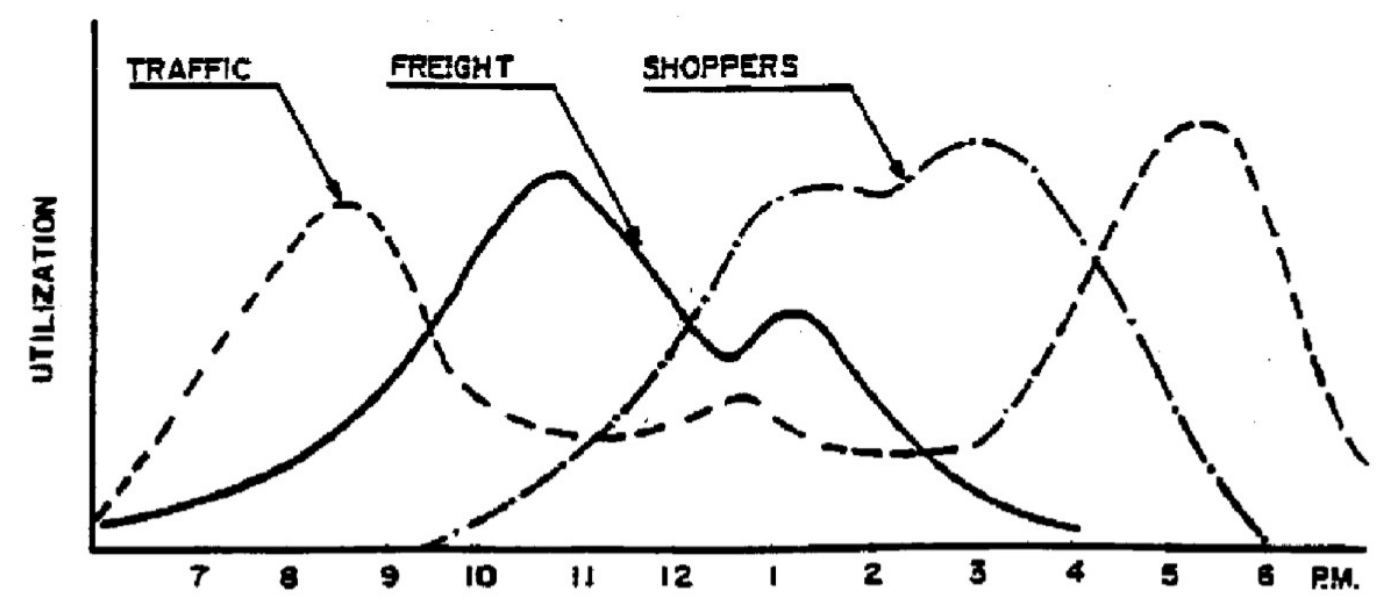

a) San Francisco (Habib, 1976)

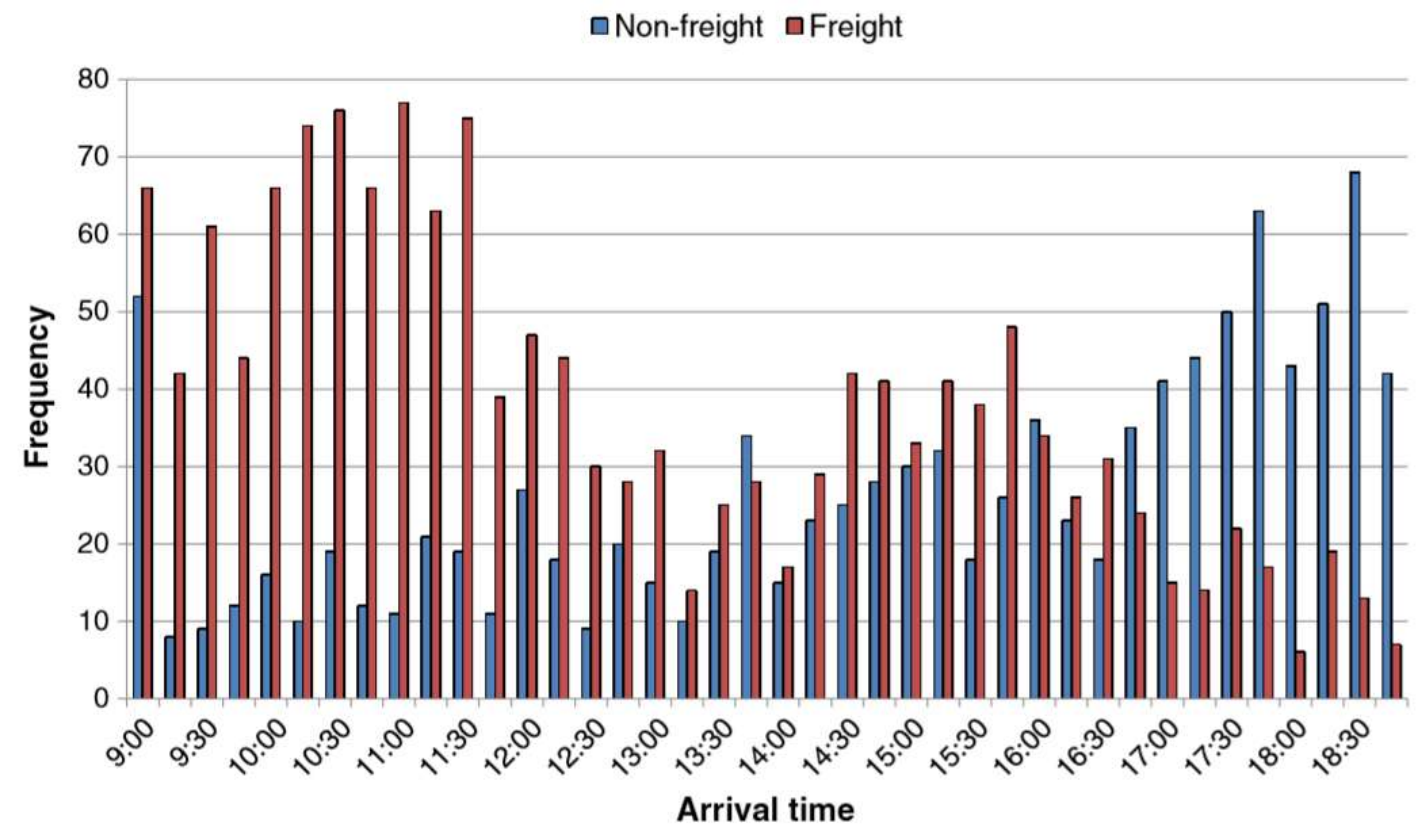

b) City of Lisbon, Portugal (Alho and e Silva, 2014)

Figure 2.1 Hourly on-street commercial parking demand from literature 
The parking adequacy problem may be more serious for service vehicles because of the restriction to park in loading/unloading zones. In some urban areas such as the City of Seattle (Washington), and the City of Baltimore (Maryland), service vehicles are not allowed to park in loading/unloading zones (City of Seattle, 2014; City of Baltimore, 2014). In addition, some service vehicles that are passenger cars are not allowed to park in loading/unloading zones. The City of Portland (Oregon) partially allows service vehicles to park in loading/unloading zones (Oregon Gov, 2012). In many cases service vehicles can park in loading zones for shorter time than delivery vehicles. However, in some cities, such as New York City (New York), and the City of Chicago (Illinois), service vehicles are allowed to park in loading/unloading zones (City of New York, 2014; City of Chicago, 2014).

\subsubsection{Parking behaviors of commercial vehicles}

When parking is not a problem for commercial vehicles, commercial vehicle drivers usually park as close to goods receivers as possible. In the central commercial area in Pusan City (South Korea), approximately half of the commercial vehicles parked in front of retail stores (Jung, 1999). Similarly, in Spain, drivers prefer to park in loading zones that are the closest to freight receivers (Muñuzuri, et al., 2012). Some drivers double park to avoid a remote area with high risk of theft and vandalism (Morris, et al., 1999). Some service vehicles need to park close to customers because it is time consuming to carry tools and, in some cases, the tools are too heavy to carry (Allen, et al., 2000).

When a nearby parking space is unavailable, drivers can respond in three ways: (a) wait in the vehicle, (b) circulate or cruise in search of available parking; and (c) double park. Several studies have assumed that commercial vehicles have multiple choices when 
responding to unavailable parking (Habib and Crowley, 1976; Aiura and Taniguchi, 2006; McLeod and Cherret, 2011); however, no studies have modeled the choice process (Figliozzi \& Tipagornwong, 2017).

\section{$\underline{\text { 2.2.3 Parking availability assessment }}$}

To identify where parking is inadequate, parking availability has to been assessed. Parking availability has been assessed with at least three approaches including demand-tosupply ratios, simulation models, and analytical models. A demand-to-supply ratio is the ratio of parking demand to parking supply; this ratio is also called the occupancy rate and can be measure on the field but the data collection is expensive. A simulation model replicates the operation of system to evaluate an existing or proposed system; simulations require demand and supply probability distributions and system parameters. An analytical model is a model that uses mathematical equation(s) to explain phenomenon or behavior (e.g. cause and effect relationships).

The demand-to-supply ratio was proposed by Jaller et al (2013) to evaluate an offpeak-hour delivery campaign in New York City by assessing commercial parking availability. The parking availability was measured with an occupancy rate that is the ratio of parking demand to parking supply. The parking demand $\left(\lambda_{c}\right)$ could be estimated with a freight trip production $\left(\lambda_{c p}\right)$ and delivery rate $\left(\lambda_{c d}\right)$, as follows, $\lambda_{c}=\lambda_{c p}+\lambda_{c d}$

The commercial vehicle parking supply or the number of on-street loading zones $\left(n_{c}\right)$ could be curb length $\left(l_{b}\right)$ divided by unit length of a loading zone $\left(l_{c}\right)$, as shown below, 
$n_{c}=\frac{l_{b}}{l_{c}}$

However, this occupancy rate model does not have any sound theoretical foundation and it is not even consistent in terms of units; these formulas have not been proven analytically and their accuracy to predicting the probability of unavailable parking is untested.

Simulation models have been used by many studies to study parking adequacy for example McLeod and Cherret (2011), Habib and Crowley (1976), and Aiura and Taniguchi (2006). The simulation model consists of several steps. The first step is to represent a transportation network and represent loading zones. The second step is to generate commercial vehicle trips based on the theoretical demand distribution (Habib and Crowley, 1976; Aiura and Taniguchi, 2006) or empirical frequency (McLeod and Cherret, 2011). The third step is to generate dwell times of commercial vehicles. The last step is to observe what type and percentage of commercial vehicles experience inadequate parking. Simulations are useful to evaluate changes and a specific setting; a severe limitation of this approach is that a simulation model has to be calibrated and does not provide general analytical solutions (e.g. optimization) or insights.

The third approach is to use an analytical model, for example models based on queuing theory ${ }^{1}$. Queuing theory explicitly models supply and demand variability and can be used to estimate parking availability. For example, Millard-Ball et al (2014) evaluated the passenger car parking availability in San Francisco utilizing empirical data and a queuing model. The empirical probability of unavailable parking spaces on a block was

\footnotetext{
${ }^{1}$ Queuing theory is the study of waiting lines of servers. The outputs of the queuing model are the expected waiting time, the expected waiting line, and the probability of a certain customers in the queuing system.
} 
estimated with the log-linear regression and the independent variables were the theoretical probability of unavailable parking spaces from the queuing theory and the number of spaces on a block. However, the method proposed by Millard-Ball et al. considered only passenger cars and is not readily transferable to assess commercial vehicle parking availability.

\subsection{Parking optimization models}

This section includes on-street parking models have been developed for commercial vehicle parking and passenger vehicle parking.

\subsubsection{Commercial parking optimization models}

The commercial parking adequacy problem has been alleviated with many techniques, such as increasing number of loading zones, relocating existing loading zones, enforcing illegal parking, shared parking, varying parking fees, and implementing a parking booking system. In some cases, multiple parking management techniques are implemented at the same time (Bomar et al., 2009; Muñuzuri et al., 2012).

Solutions to parking adequacy problems have been evaluated with optimization models (Dezi et al., 2010; Alho et al., 2016; Nourinejad and Roorda, 2016; Amer and Chow, 2016; and Muñuzuri et al., 2017). In the optimization models, the main objectives are to minimize total costs, to minimize the distance between loading zones and establishments, to maximize establishments covered by loading zones, and to maximize parking enforcement revenue.

In the parking optimization models, the decision variables are the number of loading zones, location of loading zones, parking enforcement visiting rates, and illegal parking fines. The decision variables have been proven to affect the parking behaviors of 
commercial vehicles. For example, the higher the illegal parking fine, the fewer doubleparked commercial vehicles (Nourinejad and Roorda, 2016). When there are more loading zones, fewer commercial vehicles search for parking (Muñuzuri et al., 2017) and fewer commercial vehicles double park (Amer and Chow, 2016). None of these models optimize meter rates for loading zones.

The main inputs of the optimization models are dwelling time, parking demand and supply of commercial and non-commercial vehicles, delivery and service type, commercial service cost, traffic volume, and decision making on parking options. The decision making of drivers on parking options was proposed variously, for example, choosing the cheapest options between on-street parking and double parking (Nourinejad and Roorda, 2016) and double parking when commercial parking spaces are not available (Amer and Chow, 2016).

\subsubsection{Passenger parking models}

The previous passenger parking models were developed to find optimal on-street meter rates and parking length for on-street parking. To find optimal solutions, some models use optimization models of which objective is to minimize total cost. Other models use properties of steady-state equilibrium, including flow conservation (Arnott and Inci, 2006), breakeven point between costs of parking options (Zhou, 2014), and breakeven point between departure times (Fosgerau and de Palma, 2013). The main inputs of the models are parking demand, walking distance, parking fee, parking duration, and in-transit travel time. None of these models find optimal meter rates and parking supply simultaneously.

The solutions of these models have been proven to affect parking behaviors. An increase in meter rates reduces drivers search for parking (Anderson and De Palma, 2004; 
Zhou, 2014) and changes departure times (Fosgerau and de Palma, 2013). In the other hand, if meter rates reduce slightly, the number of drivers searching for parking will increase substantially (Kobus, et al., 2013). In addition, an increase in parking supply reduces the number of drivers cruising for parking.

\subsection{Traffic delay estimation}

Traffic performance, including traffic flow rates and delays, are generally affected by traffic demand, road capacity, and road incident. Road incidents reduce road capacity, leading to a reduction in flow rate and an increase in delays.

The incident delays have been estimated with many tools. The first tool is a traffic simulation. Microscopic traffic simulations have been widely used to estimate the incident delays (Habib and Crowley, 1976; Lu and Viegas, 2007; Kladeftiras and Antoniou, 2013; Lopez et al., 2016; Alho et al., 2016). Another estimation tool is a regression model. Some of the regression models are based on field data collection (Garib et al., 1997; Han et al., 2005) while others are based on simulated data (Massahi and Cutillo, 2016).

Another delay estimation tool is a mechanistic mathematical model. Examples of these models include the deterministic queuing model (Sullivan, 1997; Li et al., 2006; Wu and Liu, 2011; Hou et al., 2012; Keegan and Gonzales, 2016), traffic shockwave theory (Wirasinghe, 1978; Cao and Menendez, 2015), the cell transmission model (Daganzo, 1994; Long et al., 2012), and queuing theory (Portilla et al., 2009; Gao and Ozbay, 2016). The deterministic queuing model analyzes delays at specific locations, for example, intersections and incident locations. Traffic shockwave theory, kinematic wave theory, and the cell transmission model analyze delays on links and corridors. 
The incident delays have been estimated with various factors. The widely used factors are traffic volume, road capacity, capacity reduction due to an incident, incident duration, and incident location on a link. The delays are a nonlinear function of traffic volume, road capacity, and capacity reduction due to an incident; the delays are a quadratic function of incident duration (Wirasinghe, 1978; Li et al., 2006; Garib et al., 1997).

Incidents have been found to reduce capacity at the incident location. At the incident, the road capacity reduction is amplified when the number of blocked lanes increases (Garib et al., 1997, Kladeftiras and Antoniou, 2013; Massahi and Cutillo, 2016). Goolsby (1971) analyzed the reduced capacity and found that an incident blocking one and two of three lanes reduced road capacity by $50 \%$ and $79 \%$, respectively. Gordon et al. (1996) found that an incident blocking 1 of 2 lanes reduced road capacity by $65 \%$. Smith et al. (2003) found that blocking one and two of three lanes resulted in capacity reduction of $63 \%$ and $77 \%$, respectively.

Incidents have also been discovered to reduce capacities at the adjacent intersections. Incidents reduce the queue dissipation rates of intersections upstream of incidents because, when queue spills over the intersections, the intersections' maximum discharge rate becomes the maximum flow rate at the incidents (Wu and Liu, 2011; Cao and Menendez, 2015). Of intersections downstream of incidents, the capacity reduction is negatively related to the distance between the intersections and the incidents (Keegan and Gonzales, 2016; Massahi and Cutillo, 2016). This is because, the longer the distance, the greater the number of vehicles in queue that are discharged with the saturation flow rate, as shown in Figure 2.2. 


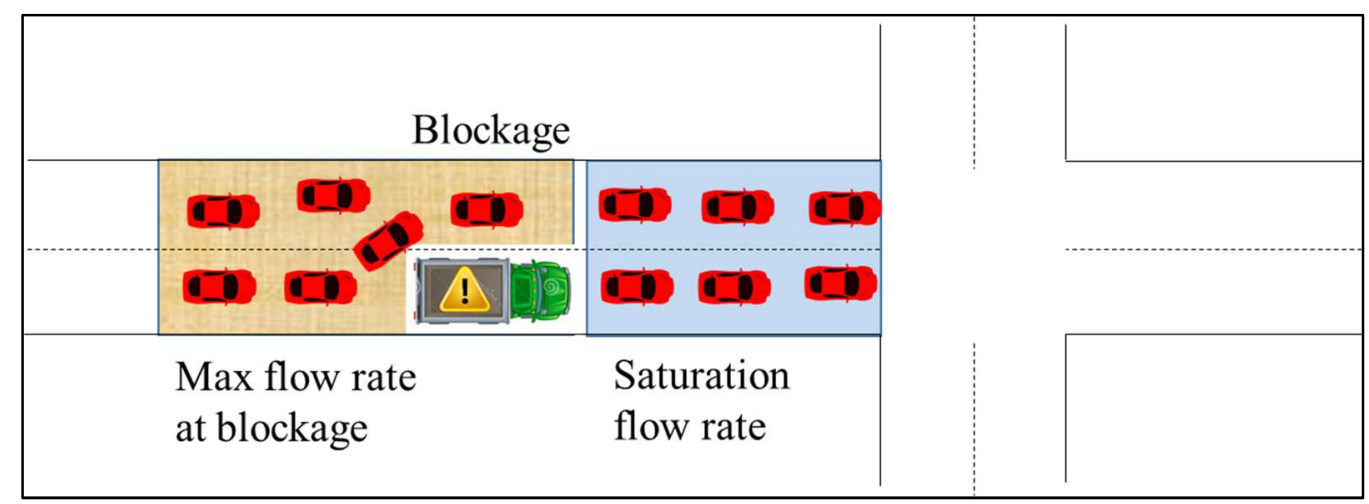

Figure 2.2 Dissipation rates nearby an intersection downstream of blockage

Delays due to incidents are affected by intersections adjacent to the incidents. The incident delays are negatively related to the distance between an incident and its adjacent intersections (Habib and Crowley, 1976; Lu and Viegas, 2007; Keegan and Gonzales, 2016). The less the distance, the more the delays are caused by incidents.

\subsection{Unique contributions}

The parking capacity problem can be alleviated with a parking optimization model. Since on-street parking systems include commercial vehicle loading zones and passenger vehicle parking spaces, the systems should be optimized for both vehicle types. From the literature, commercial parking models optimize only parking supply but do not optimize parking meter rates. None of the studies in literature include parking search cost of commercial vehicles and passenger cars, double-parking traffic delays, and double-parking fine simultaneously. These costs are driver costs and externality costs. The proposed parking optimization model fill these gaps in the literature, as shown in Table 2.1. 
Table 2.1 Commercial parking optimization models

\begin{tabular}{|l|c|c|c|c|c|c|}
\hline Study & 1 & 2 & 3 & 4 & 5 & Proposed \\
\hline $\begin{array}{l}\text { Parking options } \\
\text { On street parking }\end{array}$ & $\mathrm{x}$ & $\mathrm{x}$ & $\mathrm{x}$ & $\mathrm{x}$ & $\mathrm{x}$ & $\mathrm{x}$ \\
\hline Double parking & & $\mathrm{x}$ & $\mathrm{x}$ & & $\mathrm{x}$ & $\mathrm{x}$ \\
\hline $\begin{array}{l}\text { Decision variables } \\
\text { Number of loading zones }\end{array}$ & $\mathrm{x}$ & $\mathrm{x}$ & & $\mathrm{x}$ & $\mathrm{x}$ & $\mathrm{x}$ \\
\hline Loading zone location & $\mathrm{x}$ & $\mathrm{x}$ & & $\mathrm{x}$ & & \\
\hline Parking enforcement & & $\mathrm{x}$ & $\mathrm{x}$ & & & \\
\hline Parking fine & & & $\mathrm{x}$ & & & \\
\hline Meter rate & & & & & & $\boldsymbol{x}$ \\
\hline $\begin{array}{l}\text { Components in objective function } \\
\text { Parking enforcement cost }\end{array}$ & & & & & & \\
\hline Parking search cost & & & & $\boldsymbol{x}$ & & $\boldsymbol{x}$ \\
\hline Meter cost & & & $\mathrm{x}$ & & $\mathrm{x}$ & $\mathrm{x}$ \\
\hline Parking citation fines & & & $\mathrm{x}$ & & $\mathrm{x}$ & $\mathrm{x}$ \\
\hline Walking trolleying cost/distance & & & $\mathrm{x}$ & $\mathrm{x}$ & & \\
\hline Demand-to-supply ratio & $\mathrm{x}$ & & & $\mathrm{x}$ & & \\
\hline Establishment coverage & $\mathrm{x}$ & $\mathrm{x}$ & & & & \\
\hline Traffic delay cost due to double parking & & $\mathrm{x}$ & $\mathrm{x}$ & & & $\mathrm{x}$ \\
\hline Emission cost due to double parking & & $\mathrm{x}$ & & & & \\
\hline Passenger parking cost & & & & & $\boldsymbol{x}$ & $\boldsymbol{x}$ \\
\hline Cost of passenger travel time & & & & & $\mathrm{x}$ & \\
\hline $\begin{array}{l}\text { Factors considered } \\
\text { Delivery type }\end{array}$ & & & & & & \\
\hline Vehicle type & $\boldsymbol{x}$ & & & & & $\boldsymbol{x}$ \\
\hline Parking demand & $\mathrm{x}$ & $\mathrm{x}$ & $\mathrm{x}$ & $\mathrm{x}$ & $\mathrm{x}$ & $\mathrm{x}$ \\
\hline Walking trolleying distance & & & & & $\boldsymbol{x}$ & $\boldsymbol{x}$ \\
\hline Traffic volume & & & & & $\mathrm{x}$ & \\
\hline $\begin{array}{l}\text { Elastic passenger parking demand due to } \\
\text { delays created by a double-parking event }\end{array}$ & & & & & & \\
\hline Sort) Det & & & & \\
\hline
\end{tabular}

Source: 1) Dezi et al. (2010)

3) Nourinejad et al.(2016)

5) Amer and Chow (2016)

2) Alho et al. (2016)

4) Muñuzuri et al.(2017)

In addition, most of passenger parking models optimize parking meter rates. Only one study optimizes the number of parking spaces. However, none of the models optimizes both parking supply and parking fee simultaneously. On-street meter costs are rarely included in the passenger parking models. The proposed parking optimization model fill 
these gaps in the literature, as shown in Table 2.2. One model assumes that passenger vehicles park on street for a short time period. This assumption is reasonable because many long-term parking vehicles usually park off-street because off-street meter rates for longterm parking are usually lower than on-street meter rate.

Table 2.2 Passenger parking management models

\begin{tabular}{|c|c|c|c|c|c|c|}
\hline Study & 1 & 2 & 3 & 4 & 5 & Proposed \\
\hline $\begin{array}{l}\text { Parking option } \\
\text { On-street parking }\end{array}$ & $\mathrm{X}$ & $\mathrm{X}$ & $\mathrm{X}$ & $\mathrm{X}$ & & $\mathrm{X}$ \\
\hline Off-street parking & & & $\mathrm{x}$ & $\mathrm{x}$ & $\mathrm{x}$ & $\mathrm{x}$ \\
\hline $\begin{array}{l}\text { Method } \\
\text { Cost minimization }\end{array}$ & $\mathrm{x}$ & & & $\mathrm{x}$ & & $\mathrm{X}$ \\
\hline Flow conservation & & $\mathrm{x}$ & & & & \\
\hline Breakeven point between parking options & & & $\mathrm{x}$ & & & \\
\hline Breakeven point between departure times & & & & & $\mathrm{x}$ & \\
\hline $\begin{array}{l}\text { Optimal solutions } \\
\text { On-street parking fee }\end{array}$ & $\mathrm{X}$ & $\mathrm{x}$ & $\mathrm{x}$ & & & $\mathrm{X}$ \\
\hline Number of parking spaces & & & & $x$ & & $x$ \\
\hline Time-varying off-street parking fee & & & & & $\mathrm{x}$ & \\
\hline $\begin{array}{l}\text { Components to find optimal solutions } \\
\text { Parking search cost }\end{array}$ & $X$ & $\mathrm{X}$ & $\mathrm{X}$ & $X$ & & $X$ \\
\hline Walking cost & $\mathrm{x}$ & & & $\mathrm{x}$ & & $\mathrm{x}$ \\
\hline On-street meter cost & & & $\boldsymbol{x}$ & & & $x$ \\
\hline Off-street meter cost & & & $\mathrm{X}$ & $\mathrm{x}$ & $\mathrm{x}$ & $\mathrm{x}$ \\
\hline Location of parking spaces & $\mathrm{x}$ & & & & & \\
\hline Stock cars in transit and in parking spaces & & $\mathrm{x}$ & & & & \\
\hline Willingness to pay & & & $\mathrm{x}$ & & & \\
\hline Money metric travel utility & & & & & $\mathrm{x}$ & \\
\hline $\begin{array}{l}\text { Factors considered } \\
\text { Parking demand }\end{array}$ & $X$ & $\mathrm{X}$ & $\mathrm{X}$ & $X$ & $\mathrm{x}$ & $X$ \\
\hline Parking supply & $\mathrm{x}$ & $\mathrm{x}$ & $\mathrm{x}$ & $\mathrm{x}$ & $\mathrm{x}$ & $\mathrm{x}$ \\
\hline Different parking durations & & & & $\mathrm{x}$ & & \\
\hline Travel time & & & & & $\mathrm{x}$ & \\
\hline $\begin{array}{l}\text { Assumption } \\
\text { Short term parking (e.g. shoppers) }\end{array}$ & $x$ & & & & & $x$ \\
\hline Zero off-street parking search cost & & & $\mathrm{X}$ & $X$ & $\mathrm{x}$ & $\mathrm{x}$ \\
\hline Walking time is negligible & & $\mathrm{x}$ & & & & \\
\hline Elastic demand to trip price & & $\mathrm{x}$ & & & & \\
\hline
\end{tabular}

Source: 1) Anderson and De Palma (2004)

3) Zhou (2014)

2) Arnott and Inci (2006)

5) Fosgerau and De Palma (2013)

4) Inci and Lindsey (2015) 
Traffic incident delays have been estimated with many methods. Previous traffic delay estimations consider many factors, including traffic volume, incident duration, maximum flow rate, incident location, incident frequency, turning movement at intersections, and incident type. Some studies take into account the impact of traffic incidents on either an intersection upstream of the incidents or intersections downstream of the incidents. However, none of these studies consider the impact of incidents on both intersections upstream and downstream of the incidents simultaneously. 


\section{Chapter 3 Traffic Delays of On-Street Parking System}

\subsection{Introduction}

Traffic delays are associated with on-street parking systems. If a driver parks onstreet, the vehicle will temporarily block a traffic lane adjacent to a parking spot while the driver maneuvers the vehicle in and out of the parking spot. If a commercial driver double parks in a traffic lane, the commercial vehicle will block the traffic lane while the driver services customers. If a car driver parks off street, traffic delays will be zero since parking the car takes place in a garage or a parking lot. The traffic delays are different among parking options.

This chapter proposes a method for estimating traffic delays created by parking maneuver and double parking. This chapter contains a flow density diagram, analytical estimation of parking maneuver delays, analytical estimation of isolated double-parking delays, analytical estimation of corridor double-parking delays, a simulation for corridor double-parking traffic delay estimation, and case study for the traffic delays.

\subsection{Fundamental flow density diagram}

The traffic delays due to a parking maneuver event and a double-parking event are analyzed based on the fundamental flow-density diagram. The diagram can be described with a two-regime traffic stream model, as shown in Figure 3.1. The first regime, regime I, represents uncongested flows and the second regime, regime II, represents congested flows. Since the ratio of flow to density is constant in the first regime, speeds are constant in the uncongested flows. Traffic states that are involved in the double-parking location include arrival flow (State A), congested bottleneck flow (State B), uncongested bottleneck flow (State B'), and saturation flow (State S). 


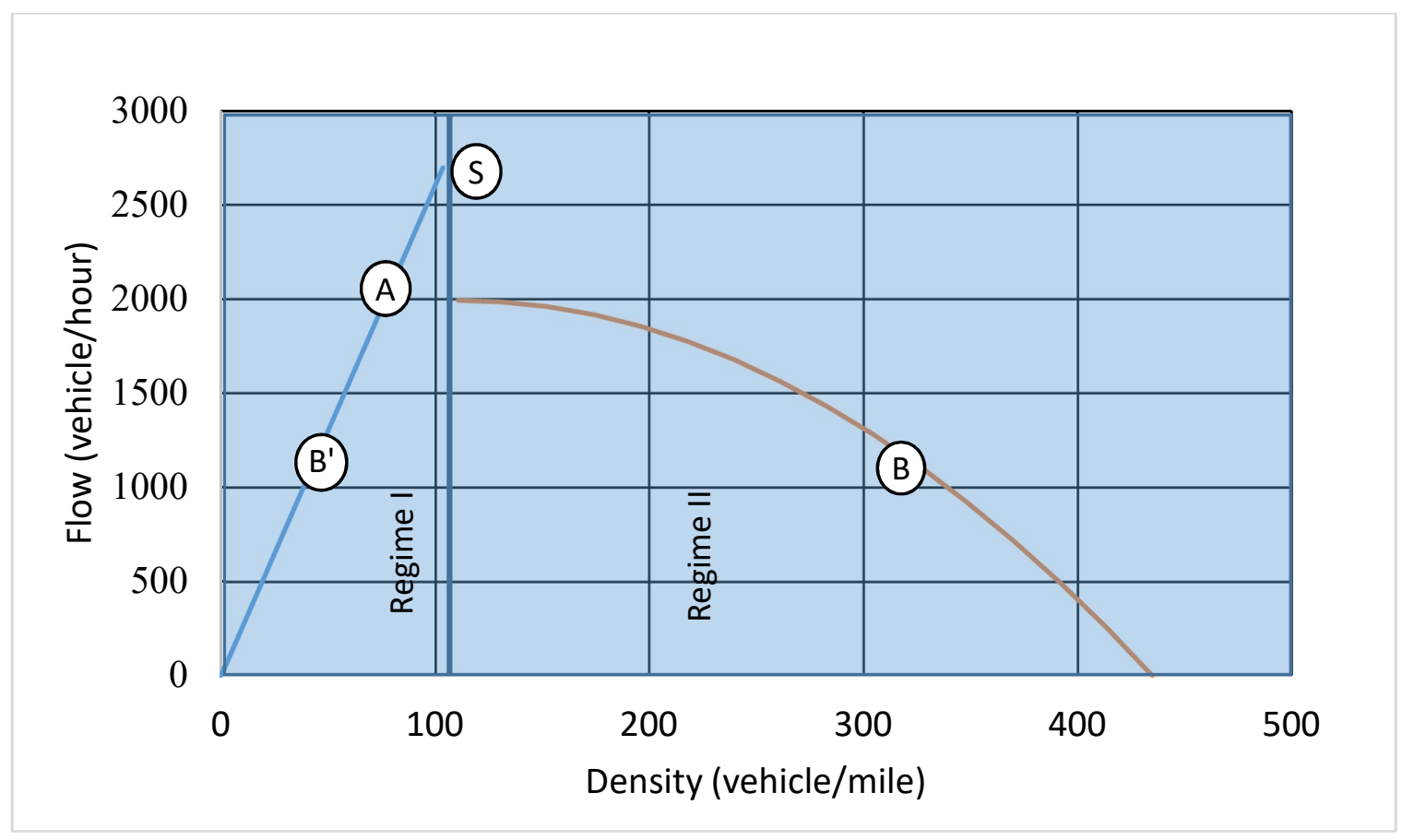

Figure 3.1 Fundamental diagram for a 2-lane roadway

\subsection{Parking maneuver delays}

Maneuver delays occur when a vehicle enters or exits a parking spot on street.

When a vehicle enters or exits a parking spot, the vehicle temporarily blocks a lane that is adjacent to the parking spot as shown in Figure 3.2.

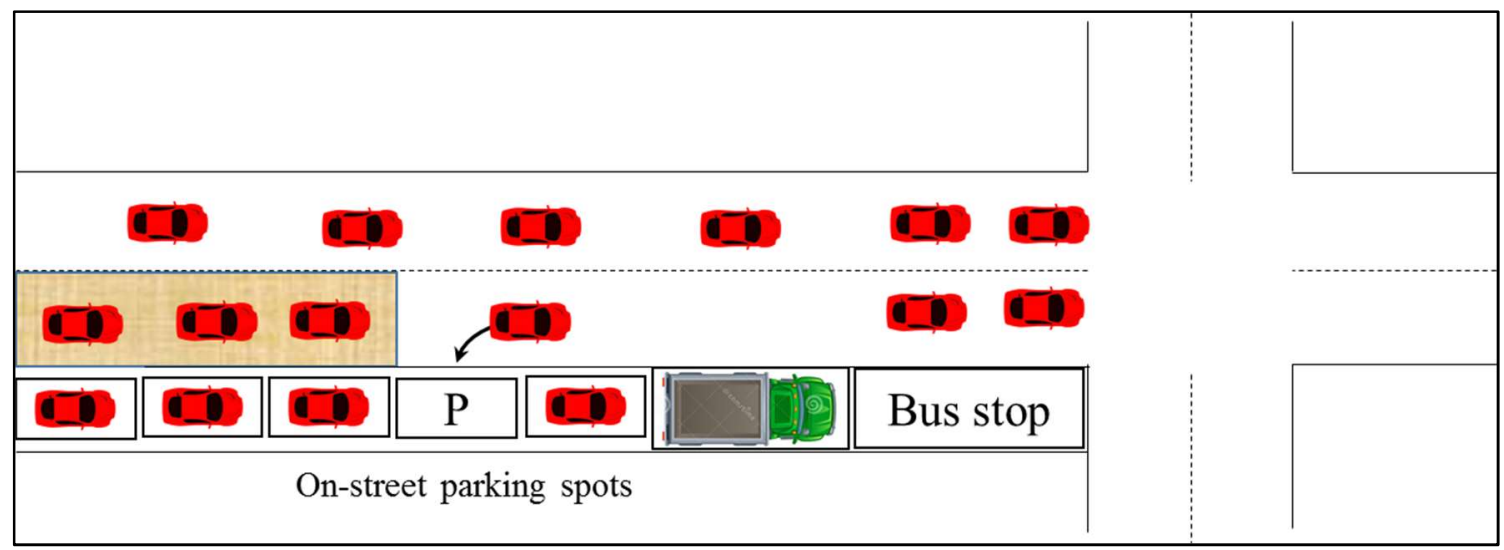

Figure 3.2 A car entering a parking space 
A deterministic queuing diagram of the lane adjacent to a car entering the parking space is shown in Figure 3.3. Since the parking maneuver takes a short time, vehicles behind the parking cars are assumed to wait until the car finishing parking - without overtaking the car (Cao and Menendez, 2015). Therefore, the flow rate equals zero $\left(q_{o}\right)$. After the car finishes parking, the flow rate becomes the saturation flow rate $\left(q_{s}\right)$ until all the queue is dissipated.

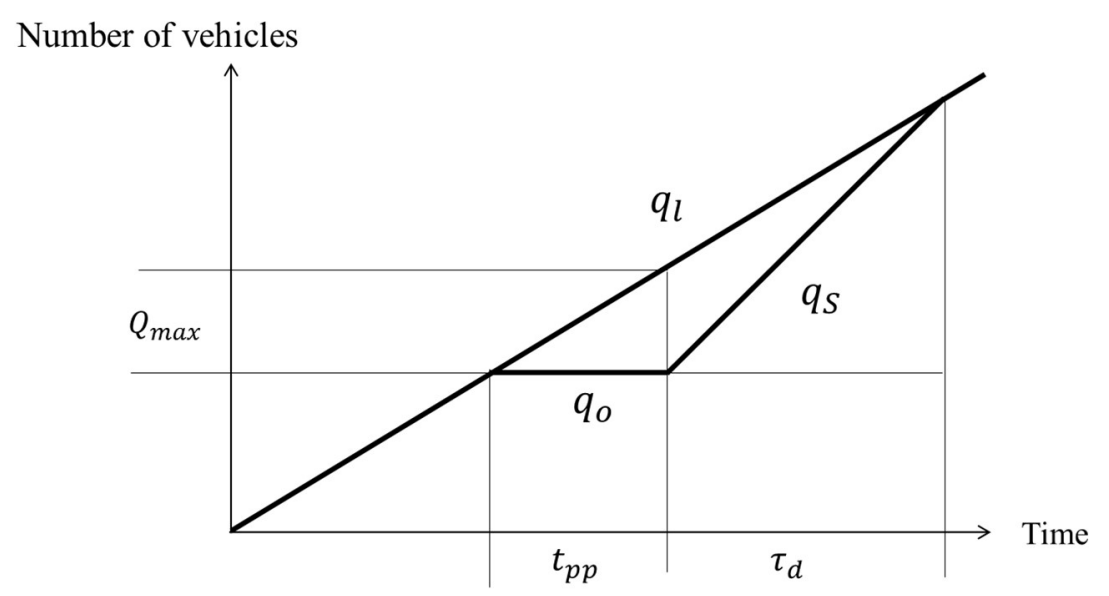

Figure 3.3 Deterministic queuing diagram for parking maneuver

The traffic delays due to the passenger car entering the parking space are estimated as follows,

- Total input during the event

$q_{l}\left(t_{p p}+\tau_{d}\right)$

○ Total output during the event

$q_{s} \tau_{d}$

- During the presence of the queue, total input equals total output

$$
q_{l}\left(t_{p p}+\tau_{d}\right)=q_{s} \tau_{d}
$$




$$
\tau_{d}=\frac{q_{l} t_{p p}}{\left(q_{S}-q_{l}\right)}
$$

○ Maximum queue length

$q_{l} t_{p p}$

- Traffic delay estimation

$$
\frac{1}{2}\left(q_{l} t_{p p}\right)\left(t_{p p}+\tau_{d}\right)=\frac{q_{l} t_{p p}}{2}\left[t_{p p}+\frac{q_{l} t_{p p}}{\left(q_{S}-q_{l}\right)}\right]=\frac{q_{l} q_{S} t_{p p}^{2}}{2\left(q_{S}-q_{l}\right)}
$$

If duration of the car vacating the parking space equals the duration of the car entering it, the traffic delays of the car vacating the parking space equal the traffic delays of the same car entering the parking space. Traffic delay cost due to a passenger car entering and vacating a parking space is shown as below,

$c_{d p}=c_{p t}\left[\frac{q_{l} q_{s} t_{p p}^{2}}{2\left(q_{S}-q_{l}\right)}+\frac{q_{l} q_{s} t_{p p}^{2}}{2\left(q_{S}-q_{l}\right)}\right]=c_{p t} \frac{q_{l} q_{s} t_{p p}^{2}}{\left(q_{S}-q_{l}\right)}$

Similar to the passenger car entering and vacating a parking space, traffic delay cost due to a commercial vehicle entering and vacating a loading zone is shown as below,

$c_{d c}=c_{p t} \frac{q_{l} q_{s} t_{p c}^{2}}{\left(q_{s}-q_{l}\right)}$

\subsection{Isolated double-parking delays}

A commercial vehicles double parks at which is very far from any intersections. The double-parking does not affect any intersections. This double-parking is isolated. The traffic queue created by a double-parking event is located using a space-time diagram. Traffic delays is estimated using a deterministic queuing diagram. Double-parking at an isolated location is a basis for double-parking in a corridor, including locating traffic queue and estimating traffic delays. 


\subsubsection{Shockwave diagram}

Let vehicle arrival be uniformly distributed and let a double-parking location be far away from any intersections. Before the double-parking event takes place, the arrival rate is lower than the road capacity; therefore, there is no queue.

When the double-parking event occurs, the road capacity reduces. If the arrival rate $\left(\mathrm{q}_{\mathrm{A}}\right)$ is higher than the maximum flow rate at the double-parking location $\left(\mathrm{q}_{\mathrm{B}}\right)$, queue will propagate backward with a shockwave speed of $\omega_{\mathrm{AB}}$. If the double-parking duration is $\tau_{b}$, the maximum queue length will be $\left(\tau_{b} \cdot \omega_{A B}\right)$. The queue does not propagate forward because the uncongested bottleneck flow rate $\left(\mathrm{q}_{\mathrm{B}}\right)$ equals the congested bottleneck flow rate $\left(\mathrm{q}_{\mathrm{B}}\right)$, as shown in Figure 3.4.

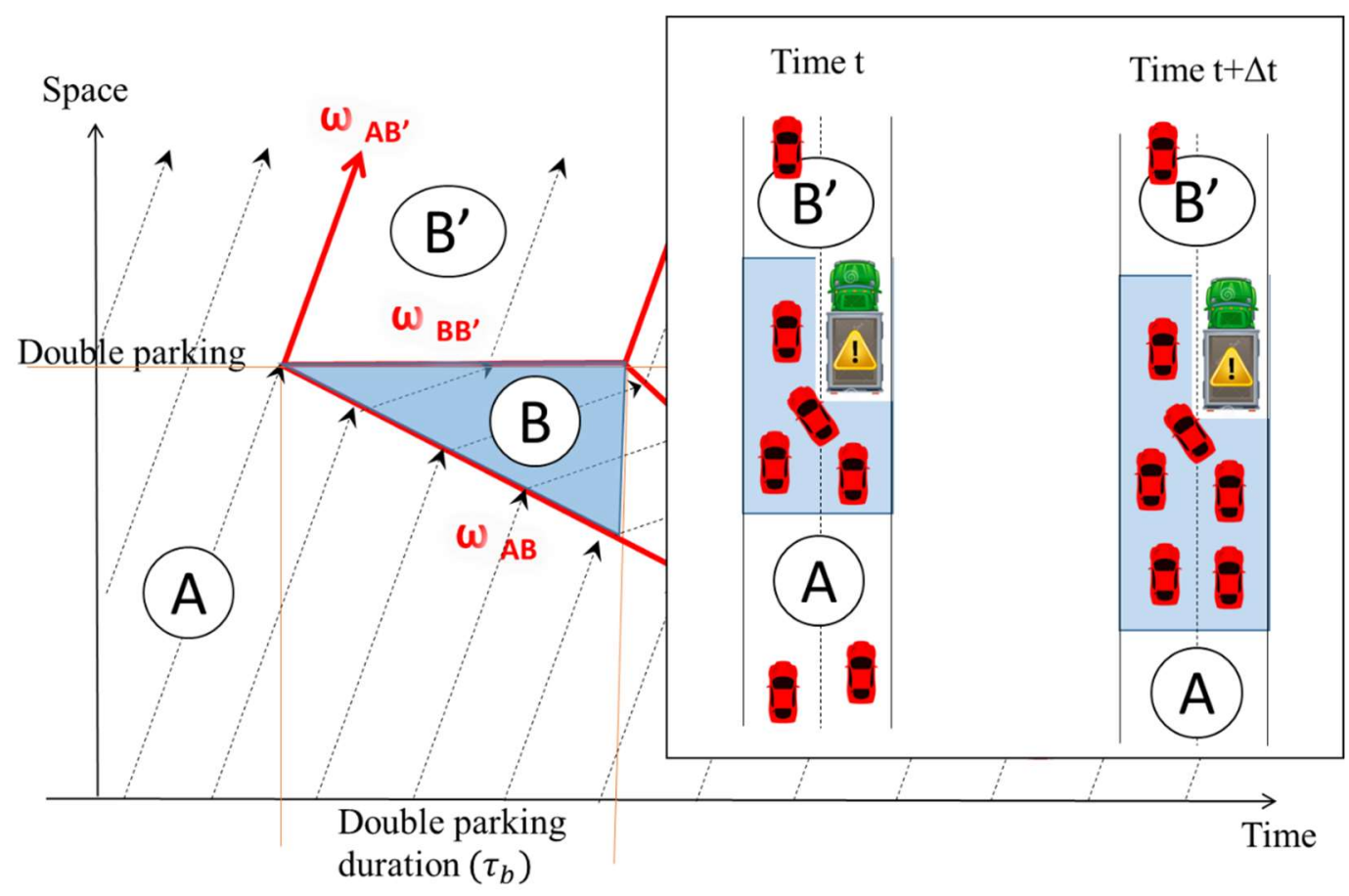

Figure 3.4 Shockwave diagram during double parking

After the vehicle stops double parking, the maximum flow rate at the doubleparking location $\left(\mathrm{q}_{\mathrm{B}}\right)$ resumes the saturation flow rate $\left(\mathrm{q}_{\mathrm{S}}\right)$. Since the discharge rate of 
$\mathrm{q}_{\mathrm{S}}$ is greater than the congested flow rate $\mathrm{q}_{\mathrm{B}}$ in the queue, the front of the queue moves backward with a shockwave speed of $\omega_{\mathrm{BS}}$. The end of the queue still moves backward with the shockwave speed of $\omega_{\mathrm{AB}}$. The shockwave speed of $\omega_{\mathrm{BS}}$ is greater than the shockwave speed of $\omega_{\mathrm{AB}}$; therefore, the queue front becomes closer to the queue end. Eventually, the queue completely dissipates, as shown in Figure 3.5.

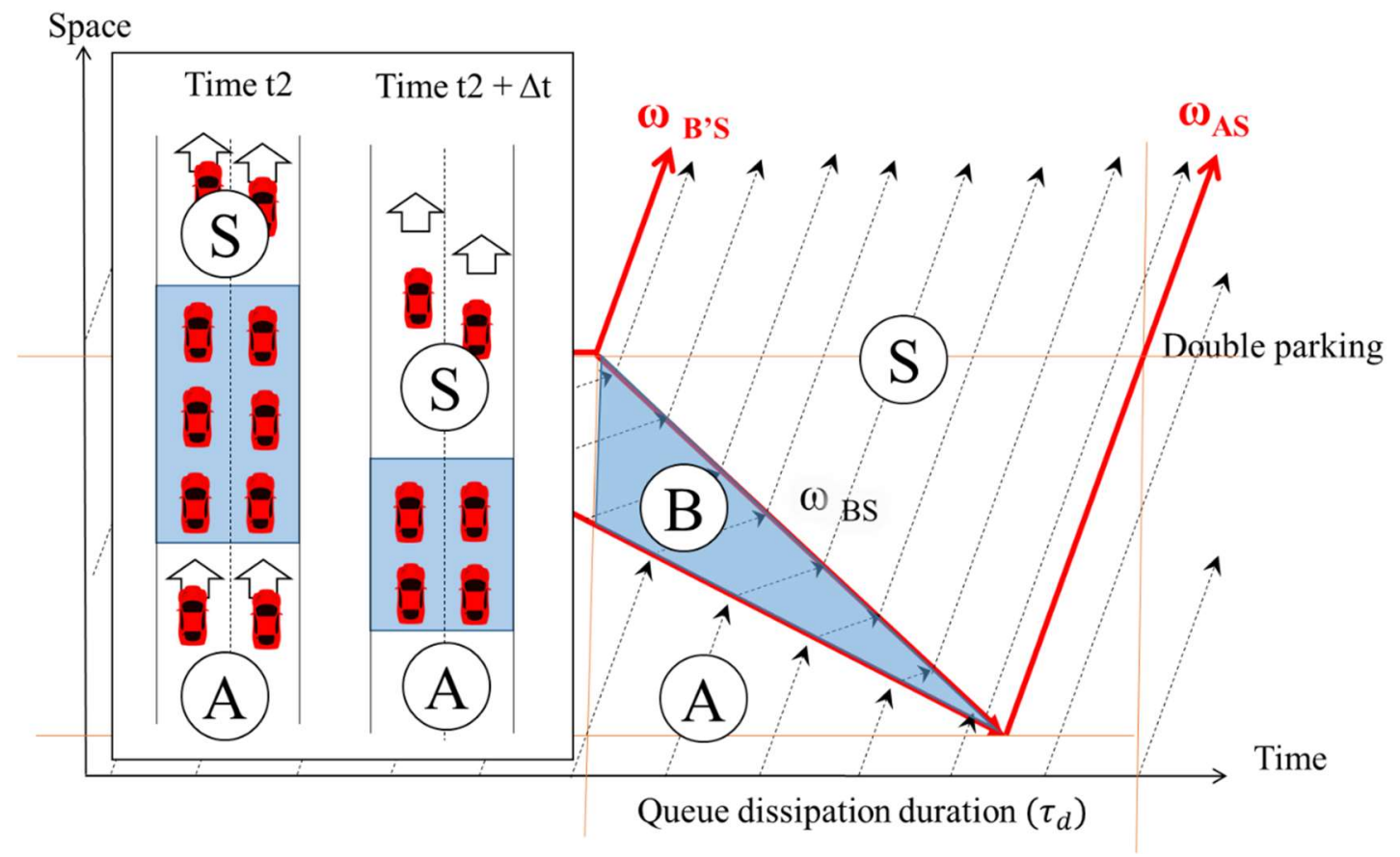

Figure 3.5 Shockwave diagram after double parking ends

\subsubsection{Traffic delay estimation}

The total delays of the isolated double parking are estimated using a deterministic queuing diagram. Let vehicle arrival be uniformly distributed and one lane be blocked by a commercial vehicle. If the commercial vehicle blocks the lane for a period of $\tau_{b}$, $a$ deterministic queuing diagram will be plotted as shown in Figure 3.6. 
Number of vehicles

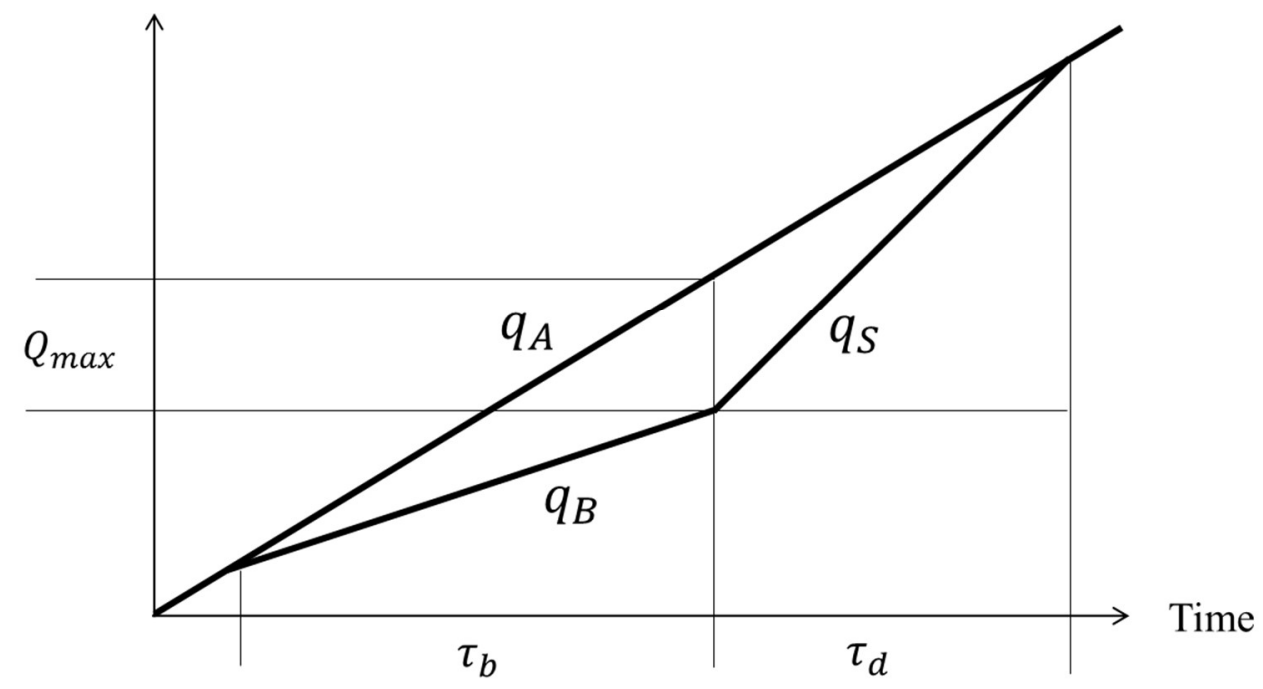

Figure 3.6 Deterministic queuing diagram of an isolated double parking

During the whole double-parking event, total input equals total output

$q_{A}\left(\tau_{b}+\tau_{d}\right)=q_{B} \tau_{b}+q_{S} \tau_{d}$

$\left(q_{A}-q_{B}\right) \tau_{b}=\left(q_{S}-q_{A}\right) \tau_{d}$

$\tau_{d}=\left(\frac{q_{A}-q_{B}}{q_{S}-q_{A}}\right) \tau_{b}$

The total delays $(d)$ is expressed as follow,

$$
\begin{aligned}
& d=\frac{1}{2}\left(q_{A}-q_{B}\right) \tau_{b}\left(\tau_{b}+\tau_{d}\right) \\
& d=\frac{1}{2}\left(q_{A}-q_{B}\right) \tau_{b}\left[\tau_{b}+\left(\frac{q_{A}-q_{B}}{q_{S}-q_{A}}\right) \tau_{b}\right] \\
& d=\frac{\left(q_{S}-q_{B}\right)\left(q_{A}-q_{B}\right) \tau_{b}^{2}}{2\left(q_{S}-q_{A}\right)}
\end{aligned}
$$

\subsection{Corridor double parking delays}

A commercial vehicle often double parks in a city center. In many city centers, spacing between intersections is very short. Intersections are so close that traffic flow and 
queue at one intersection might affect traffic flows and queues at other intersections. Double parking reduces a roadway capacity, resulting in a traffic queue. Since the doubleparking takes place close to intersections, the queue might spill back to an intersection upstream of the double-parking event. The queue reduces the capacity of the intersection. The queue might also change distribution of traffic approaching an intersection downstream of the double-parking event.

\section{$\underline{\text { 3.5.1 Intersections for estimating double-parking traffic delays }}$}

For estimating traffic delays due to double-parking in a corridor, multiple locations are taken account, including an entry intersection, a double-parking commercial vehicle, an intersection upstream of the vehicle, and an intersection downstream of the vehicle. A map of the intersections and the double-parking vehicle is shown in Figure 3.7.

Traffic enters a corridor at the entry intersection. Traffic passes the upstream intersection before approaching the double-parking location. After passing the doubleparking location, traffic is going to the downstream intersection. Signals in the corridor are assumed to be exceptionally coordinated. 


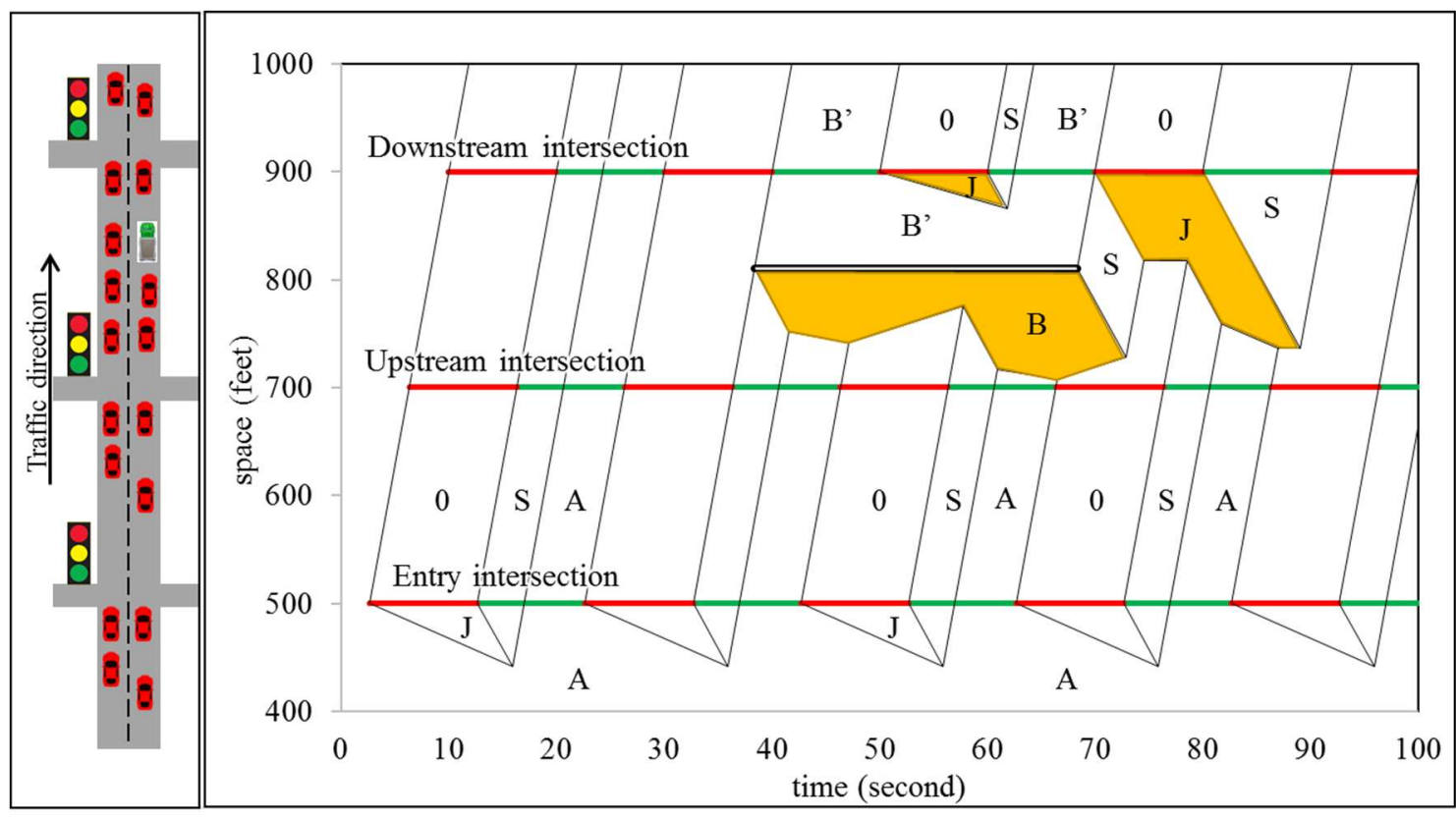

$\mathrm{A}=$ Arrival flow $\quad \mathrm{B}=\mathrm{B}^{\prime}=$ Discharge flow at double parking

$\mathrm{S}=$ Saturation flow $\quad 0=$ Zero flow $\quad \mathrm{J}=\mathrm{Jam}$

Traffic delays created by a double parking event

Figure 3.7 Shockwave diagram of a double-parking event in a corridor

\subsubsection{Entry intersection}

Traffic enters a corridor at the entry intersection. At the entry intersection, the arrival flow is uniformly distributed. Traffic signal at this intersection changes the distribution. During the red lights, vehicles stop; therefore, traffic flow rates are zero $\left(q_{0}\right)$. Queue propagates. In the beginnings of green times, the flow rate is saturation flow rate $\left(q_{s}\right)$ when the queue dissipates. After the queue is cleared, the flow rate becomes the arrival flow rate $\left(q_{A}\right)$, as shown in Figure 3.7. A queuing diagram of the entry intersection is shown in Figure 3.8. 


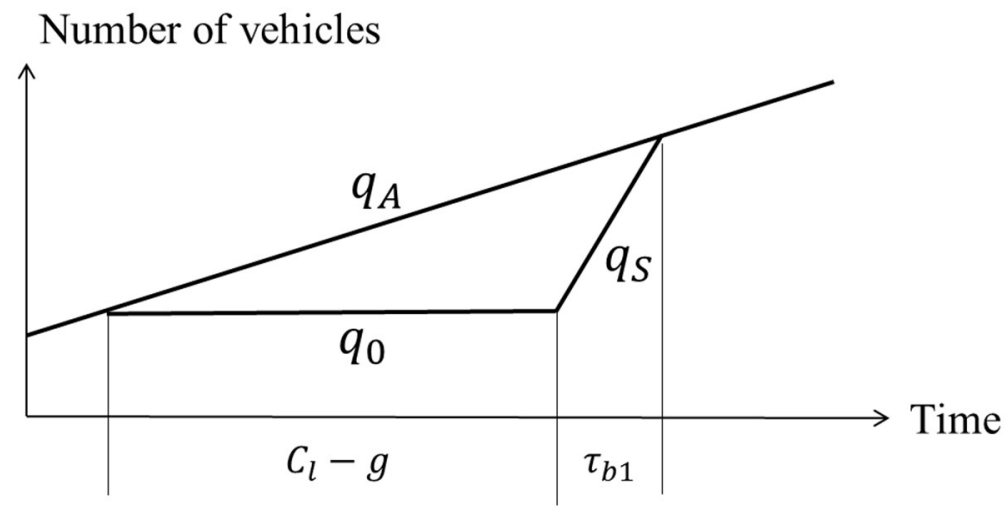

Figure 3.8 Queuing diagram of the entry intersection of a corridor

From Figure 3.8, the duration of $q_{0}$ equals the cycle length $\left(C_{l}\right)$ subtracted by the green time $(g)$. The duration of $q_{S}$ equals $\tau_{b 1}$. The duration of $q_{A}$ equals duration of $q_{0}$ plus the duration of $q_{s}$. The duration $\tau_{b 1}$ is estimated below,

- Total input during the queue

$$
q_{A}\left(C_{l}-g+\tau_{b 1}\right)
$$

○ Total output during the queue

$$
q_{s} \tau_{b 1}
$$

$\circ$ During the presence of the queue, total input equals total output

$$
\begin{aligned}
& q_{A}\left(C_{l}-g+\tau_{b 1}\right)=q_{S} \tau_{b 1} \\
& \tau_{b 1}=\frac{q_{A}\left(C_{l}-g\right)}{q_{S}-q_{A}}
\end{aligned}
$$

The departure flows of the entry intersection are the arrival flows of the double-parking location, as shown in Figure 3.7.

\subsubsection{Upstream intersection}

The upstream intersection is an intersection upstream of a double-parking commercial vehicle. Arrival flows of the upstream intersection are departure flows of the 
entry intersection. If a commercial vehicle does not double park, delays at the upstream intersection will be zero since all the intersections are exceptionally coordinated, as shown in Figure 3.7.

When a commercial vehicle double parks, it causes traffic congestion and queue. If the arrival rate of the upstream intersection is more than the flow rate at the double-parking location $\left(q_{B}\right)$, the queue at the double-parking location will propagate and spill back to the upstream intersection.

\subsubsection{Double-parking location}

At the double-parking location, the maximum flow rate equals $\left(q_{B}\right)$. From Figure 3.7, if the arrival rate is saturation flow rate $\left(q_{S}\right)$, the propagation rate will be the highest. If the arrival rate is $q_{A}$, the queue will still propagate since the arrival flow rate $\left(q_{A}\right)$ is assumed to be higher than $q_{B}$. However, during red times, queue dissipates since the arrival rate is zero. Nevertheless, overtime, the queue tends to propagate.

The queue propagation during the first green of double-parking duration is longer than the queue propagation during the other greens. This is because total departure of the first green $\left(q_{B} g\right)$ is lower than total departures of the other greens $\left(q_{B} C_{l}\right)$, as shown in Figure 3.7.

After the double-parking vehicle leaves, the departure rate becomes an intersection capacity $\left(q_{C}\right)$. Since $q_{C}$ is higher than the arrival rate $\left(q_{A}\right)$, the queue dissipates. The queuing diagram of the corridor double parking event is shown in Figure 3.9. 


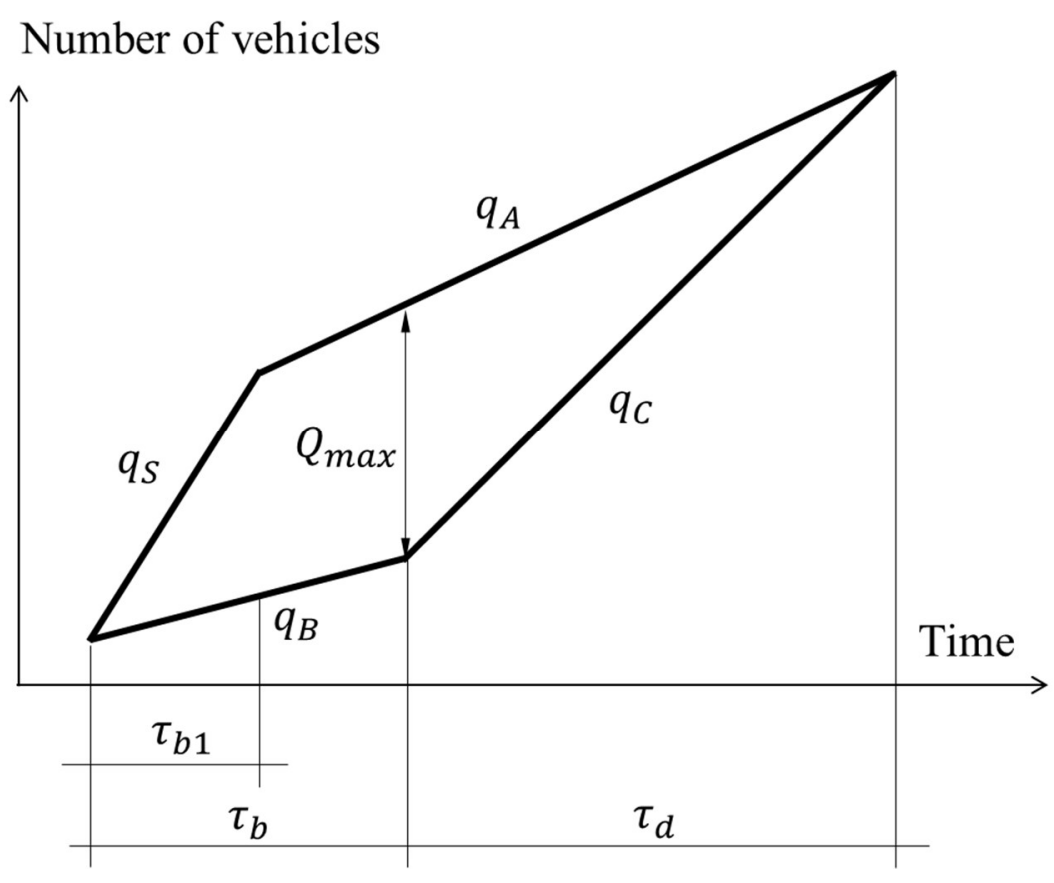

Figure 3.9 Queue diagram for corridor double parking

\section{$\underline{\text { 3.5.5 Traffic delays of one double-parking event }}$}

Traffic delays due to a commercial vehicle double parking in a corridor is estimated using Figure 3.9 as shown below,

○ Queue length at time $\tau_{b 1}\left(Q\left(\tau_{b 1}\right)\right)$

$$
\begin{aligned}
& Q\left(\tau_{b 1}\right)=\left(q_{S}-q_{B}\right) \tau_{b 1} \\
& \text { Since } \tau_{b 1}=\frac{q_{A}\left(C_{l}-g\right)}{q_{S}-q_{A}} \text { from Eq (3.6) } \\
& Q\left(\tau_{b 1}\right)=\frac{q_{A}\left(q_{S}-q_{B}\right)\left(C_{l}-g\right)}{q_{S}-q_{A}}
\end{aligned}
$$


- Maximum queue length $\left(Q_{\max }\right)$

$$
\begin{aligned}
& Q_{\text {max }}=\left(q_{S}-q_{B}\right) \tau_{b 1}+\left(q_{A}-q_{B}\right)\left(\tau_{b}-\tau_{b 1}\right) \\
& Q_{\text {max }}=\left(q_{A}-q_{B}\right) \tau_{b}+\left(q_{S}-q_{A}\right) \tau_{b 1} \\
& \text { Since } \tau_{b 1}=\frac{q_{A}\left(C_{l}-g\right)}{q_{S}-q_{A}} \text { from } E q(3.6) \\
& Q_{\max }=\left(q_{A}-q_{B}\right) \tau_{b}+q_{A}\left(C_{l}-g\right)
\end{aligned}
$$

$\circ$ Queue dissipation time $\left(\tau_{d}\right)$

During the whole double parking event, total input equals total output

$Q_{\max }+q_{A} \tau_{d}=q_{C} \tau_{d}$

$\frac{Q_{\max }}{q_{C}-q_{A}}=\tau_{d}$

Since $Q_{\max }=\left(q_{A}-q_{B}\right) \tau_{b}+q_{A}\left(C_{l}-g\right)$ from $E q(3.8)$

$$
\tau_{d}=\frac{\left(q_{A}-q_{B}\right) \tau_{b}+q_{A}\left(C_{l}-g\right)}{q_{C}-q_{A}}
$$

- Traffic delays during queue propagation with arrival flow of $q_{S}$

$$
\frac{1}{2}\left[0+Q\left(\tau_{b 1}\right)\right] \tau_{b 1}
$$

From equations $E q(3.6)$ and $E q(3.7)$,

$$
\begin{aligned}
& \frac{1}{2}\left[0+Q\left(\tau_{b 1}\right)\right] \tau_{b 1}=\frac{1}{2}\left[0+\frac{q_{A}\left(q_{S}-q_{B}\right)\left(C_{l}-g\right)}{q_{S}-q_{A}}\right] \frac{q_{A}\left(C_{l}-g\right)}{q_{S}-q_{A}} \\
& \frac{1}{2}\left[0+Q\left(\tau_{b 1}\right)\right] \tau_{b 1}=\frac{q_{A}^{2}\left(q_{S}-q_{B}\right)\left(C_{l}-g\right)^{2}}{2\left(q_{S}-q_{A}\right)^{2}} \quad E q(3.10
\end{aligned}
$$


- Traffic delays during queue propagation with arrival flow of $q_{A}$

$$
\frac{1}{2}\left[Q_{\max }+Q\left(\tau_{b 1}\right)\right]\left(\tau_{b}-\tau_{b 1}\right)
$$

From equations $E q(3.6) E q(3.7)$ and $E q(3.8)$,

$$
\begin{aligned}
& =\frac{1}{2}\left[\left(q_{A}-q_{B}\right) \tau_{b}+q_{A}\left(C_{l}-g\right)+\frac{q_{A}\left(q_{S}-q_{B}\right)\left(C_{l}-g\right)}{q_{S}-q_{A}}\right]\left[\tau_{b}-\frac{q_{A}\left(C_{l}-g\right)}{q_{S}-q_{A}}\right] \\
& =\frac{1}{2}\left[\left(q_{A}-q_{B}\right) \tau_{b}+\frac{q_{A}\left(2 q_{S}-q_{B}-q_{A}\right)\left(C_{l}-g\right)}{q_{S}-q_{A}}\right]\left[\tau_{b}-\frac{q_{A}\left(C_{l}-g\right)}{q_{S}-q_{A}}\right] \\
& =\frac{\left(q_{A}-q_{B}\right) \tau_{b}^{2}}{2}+q_{A} \tau_{b}\left(C_{l}-g\right)-\frac{q_{A}^{2}\left(2 q_{S}-q_{B}-q_{A}\right)\left(C_{l}-g\right)^{2}}{2\left(q_{S}-q_{A}\right)^{2}}
\end{aligned}
$$

- Traffic delays during queue dissipation

$\frac{Q_{\max } \tau_{d}}{2}$

From equations $E q(3.8)$ and $E q(3.9)$,

$$
\begin{aligned}
& =\frac{\left[\left(q_{A}-q_{B}\right) \tau_{b}+q_{A}\left(C_{l}-g\right)\right]^{2}}{2\left(q_{C}-q_{A}\right)} \\
& =\frac{\left(q_{A}-q_{B}\right)^{2} \tau_{b}^{2}+2 q_{A}\left(q_{A}-q_{B}\right) \tau_{b}\left(C_{l}-g\right)+q_{A}^{2}\left(C_{l}-g\right)^{2}}{2\left(q_{C}-q_{A}\right)} \\
& =\frac{\left(q_{A}-q_{B}\right)^{2} \tau_{b}^{2}}{2\left(q_{C}-q_{A}\right)}+\frac{q_{A}\left(q_{A}-q_{B}\right) \tau_{b}\left(C_{l}-g\right)}{\left(q_{C}-q_{A}\right)}+\frac{q_{A}^{2}\left(C_{l}-g\right)^{2}}{2\left(q_{C}-q_{A}\right)}
\end{aligned}
$$


- Total delays

From equations $E q(3.10) E q(3.11)$ and $E q(3.12)$,

$$
\begin{gathered}
d=\frac{q_{A}^{2}\left(q_{S}-q_{B}\right)\left(C_{l}-g\right)^{2}}{2\left(q_{S}-q_{A}\right)^{2}}+\frac{\left(q_{A}-q_{B}\right) \tau_{b}^{2}}{2}+q_{A} \tau_{b}\left(C_{l}-g\right) \\
-\frac{q_{A}^{2}\left(2 q_{S}-q_{B}-q_{A}\right)\left(C_{l}-g\right)^{2}}{2\left(q_{S}-q_{A}\right)^{2}}+\frac{\left(q_{A}-q_{B}\right)^{2} \tau_{b}^{2}}{2\left(q_{C}-q_{A}\right)} \\
+\frac{q_{A}\left(q_{A}-q_{B}\right) \tau_{b}\left(C_{l}-g\right)}{\left(q_{C}-q_{A}\right)}+\frac{q_{A}^{2}\left(C_{l}-g\right)^{2}}{2\left(q_{C}-q_{A}\right)} \\
d=\left[\frac{\left(q_{A}-q_{B}\right) \tau_{b}^{2}}{2}+\frac{\left(q_{A}-q_{B}\right)^{2} \tau_{b}^{2}}{2\left(q_{C}-q_{A}\right)}\right]+\left[q_{A} \tau_{b}\left(C_{l}-g\right)+\frac{q_{A}\left(q_{A}-q_{B}\right) \tau_{b}\left(C_{l}-g\right)}{\left(q_{C}-q_{A}\right)}\right] \\
+\left[\frac{q_{A}^{2}\left(q_{S}-q_{B}\right)\left(C_{l}-g\right)^{2}}{2\left(q_{S}-q_{A}\right)^{2}}-\frac{q_{A}^{2}\left(2 q_{S}-q_{B}-q_{A}\right)\left(C_{l}-g\right)^{2}}{2\left(q_{S}-q_{A}\right)^{2}}+\frac{q_{A}^{2}\left(C_{l}-g\right)^{2}}{2\left(q_{C}-q_{A}\right)}\right] \\
d=\frac{\left(q_{C}-q_{B}\right)\left(q_{A}-q_{B}\right) \tau_{b}^{2}}{2\left(q_{C}-q_{A}\right)}+\frac{q_{A}\left(q_{C}-q_{B}\right) \tau_{b}\left(C_{l}-g\right)}{\left(q_{C}-q_{A}\right)}+\frac{q_{A}^{2}\left(q_{S}-q_{C}\right)\left(C_{l}-g\right)^{2}}{2\left(q_{C}-q_{A}\right)\left(q_{S}-q_{A}\right)}
\end{gathered}
$$

The traffic delay formula, $E q(3.13)$, shows that traffic delays are positively related to traffic volume $\left(q_{A}\right)$, double-parking duration $\left(\tau_{b}\right)$, and red time of traffic signal $\left(C_{l}-g\right)$. However, the traffic delays are negatively related to the maximum flow rate at a double-parking location $\left(q_{B}\right)$.

In addition, the first term of the corridor delay formula, $E q(3.13)$, is similar to the isolated delay formula, $E q(3.5)$. This infers that the second and third terms of the corridor formula are the impact of a double-parking event occurring in a corridor. 
From Figure 3.7, if a double-parking event starts after the queue from signal at the entry intersection dissipates, the queue propagation rate will be constant, which is the arrival rate $\left(q_{A}\right)$ minus the maximum flow rate at the double-parking location $\left(q_{B}\right)$. The second and third terms of $E q(3.13)$ will disappear.

\subsection{Simulation for estimating double-parking delays}

Another approach to estimate traffic delays due to a double-parking event in a corridor is a simulation. For this study, the simulation is based on a deterministic queuing model and is coded with R. The script is shown in Appendix B. Advantage of a simulation over an analytical model is to that all variables, including arrival rates and signal timing, can change overtime.

There are multiple steps to acquire the traffic delays. The first step is to set locations of intersections and a double-parking commercial vehicle. The second step is to assume the traffic arrival rate to a corridor $\left(q_{A}\right)$, signal timing, and double-parking duration. The third step is to generate $q_{A}$ and to let traffic get through the intersections and the doubleparking vehicle. The last step is to observe how long vehicles are in queue at all locations. Traffic density is also considered in order to check if queue spills back and blocks upstream intersections, as shown in Figure 3.10. 


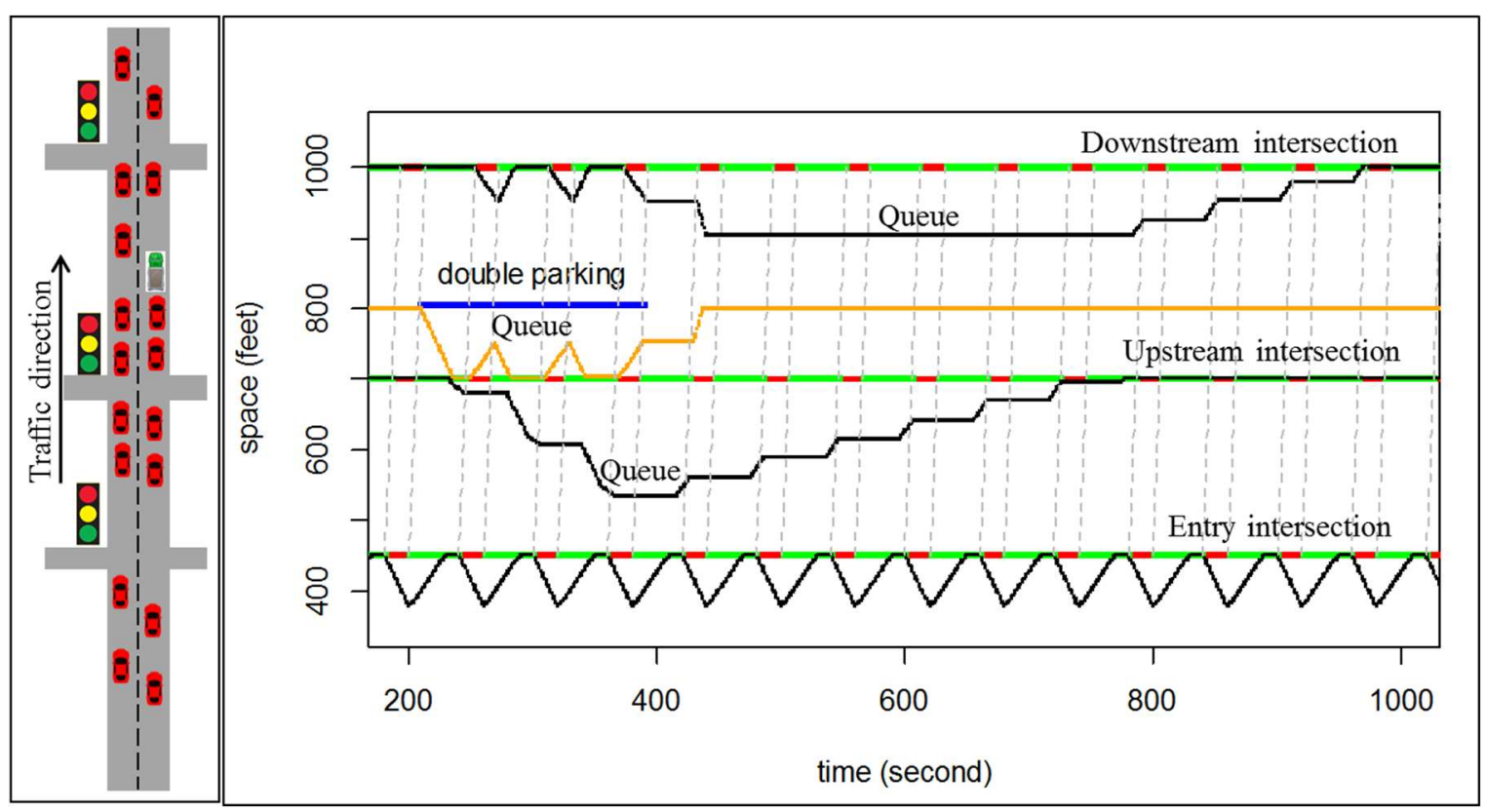

Figure 3.10 Deterministic queuing diagram from the simulation

From Figure 3.10, after the event starts, queue at the double-parking location propagates and then spills over to an intersection upstream of the double-parking location. Then queue at the upstream intersection propagates while queue at the double-parking location stops propagating.

Then, when traffic signals at all the intersections turn red, no traffic arrives at the upstream intersection; therefore, the queue at the upstream intersection is constant. At the double-parking location, there are no traffic arrivals but traffic keep passing the double parking location. As a result, the queue at the double-parking location dissipates. The traffic departing the double-parking location is the traffic arriving an intersection downstream of the double-parking location. Therefore, the queue at the downstream intersection propagates. 
After the double-parking vehicle leaves, the queue at the double-parking location dissipates. Then, the queue at the upstream intersection dissipates. After the queue at the upstream intersection fully dissipates, the queue at the downstream intersection dissipates.

\subsection{Case study for traffic delays}

\subsubsection{Base data}

The base data for estimating the traffic delays include road capacity, traffic signal, traffic volume, and double-parking characteristics. For the road capacity, saturation flow rate is estimated using the Highway Capacity Manual formula. Assumption for the estimation is that the street class is IV for urban arterials and the area type is a central business district. The road is two-lane and one-way, which is common for urban corridors. Traffic signals are exceptionally coordinated, which allows traffic flow without stopping at signalized intersections, as shown in Table 3.1.

Table 3.1 Base data for traffic delay estimation due to a double-parking event

\begin{tabular}{|l|c|c|}
\hline Parameters & Unit & Value \\
\hline Saturation flow rate & $\begin{array}{c}\text { Vehicles per hour per } \\
\text { lane }\end{array}$ & 1500 \\
\hline Number of lanes in one direction & Lanes & 2 \\
\hline Signal cycle length & Seconds & 60 \\
\hline Green time & Seconds & 40 \\
\hline Lane group capacity & Vehicles per hour & 2000 \\
\hline Maximum flow rate at a double parking & Vehicles per hour & 1270 \\
\hline Traffic volume & Vehicles per hour & $1300-1600$ \\
\hline Traffic signal coordination & - & Exceptional \\
\hline Double parking location & - & Mid block \\
\hline
\end{tabular}

The characteristics of double-parking events include duration, road capacity reduction, and location. When a vehicle is double parked, it blocks one traffic lane that 
reduces a capacity of 2 -lane road by $60 \%$. In the other words, the remaining road capacity is $40 \%$. Double parking is assumed to occur at a midblock.

\subsubsection{Outputs of case study}

The inputs to the on-street parking optimization models from this chapter are traffic delay costs. The traffic delay costs are costs due to the parking maneuver traffic delays (Section 3.3), the corridor double-parking delays (Section 3.5) and the corridor double parking delays per one vehicle (Section 3.5).

The parking maneuver traffic delay cost is estimated based on the parking maneuver time. According to equations $E q(3.2)$ and $E q(3.3)$ and the data from Table 3.1, the parking maneuver traffic delay cost of a passenger car $\left(c_{d p}\right)$ and a commercial vehicle $\left(c_{d c}\right)$ are estmated, as shown in Figure 3.11 and Figure 3.12, respectively. The maneuver time of a passenger car, entering or leaving a parking space, is assumed to be 9 seconds per maneuver which is the average value from data collection (Cao, et al., 2014). The parking maneuver time of a commercial vehicle is assumed to be a little higher than the time of a passenger car, say 20 seconds. If the traffic volume per lane is 700 vehicle per hour, $c_{d p}$ and $c_{d c}$ will equal $\$ 0.13$ and $\$ 0.65$ per maneuver, respectively. 


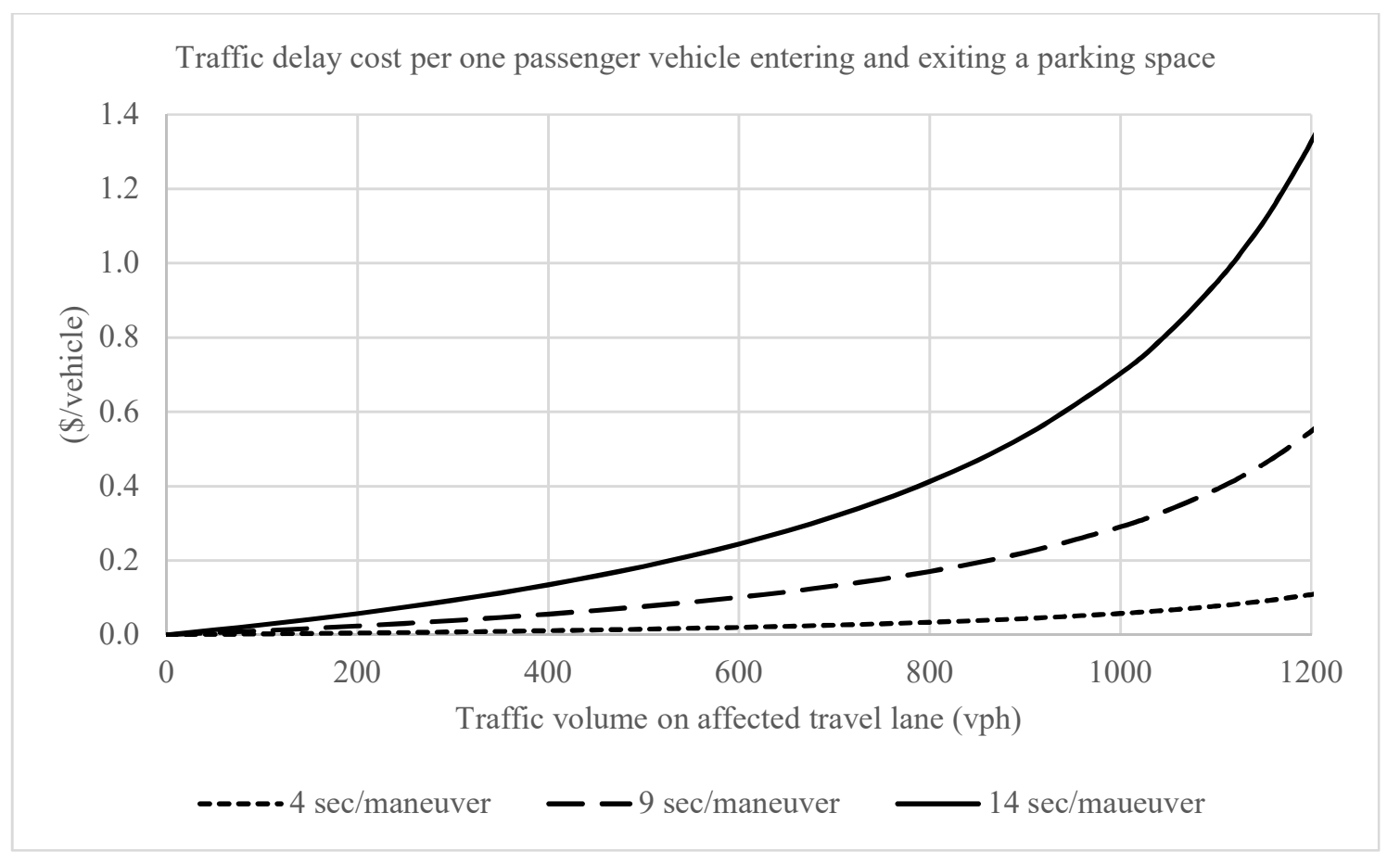

Figure 3.11 Traffic parking-maneuver delay cost of a passenger car

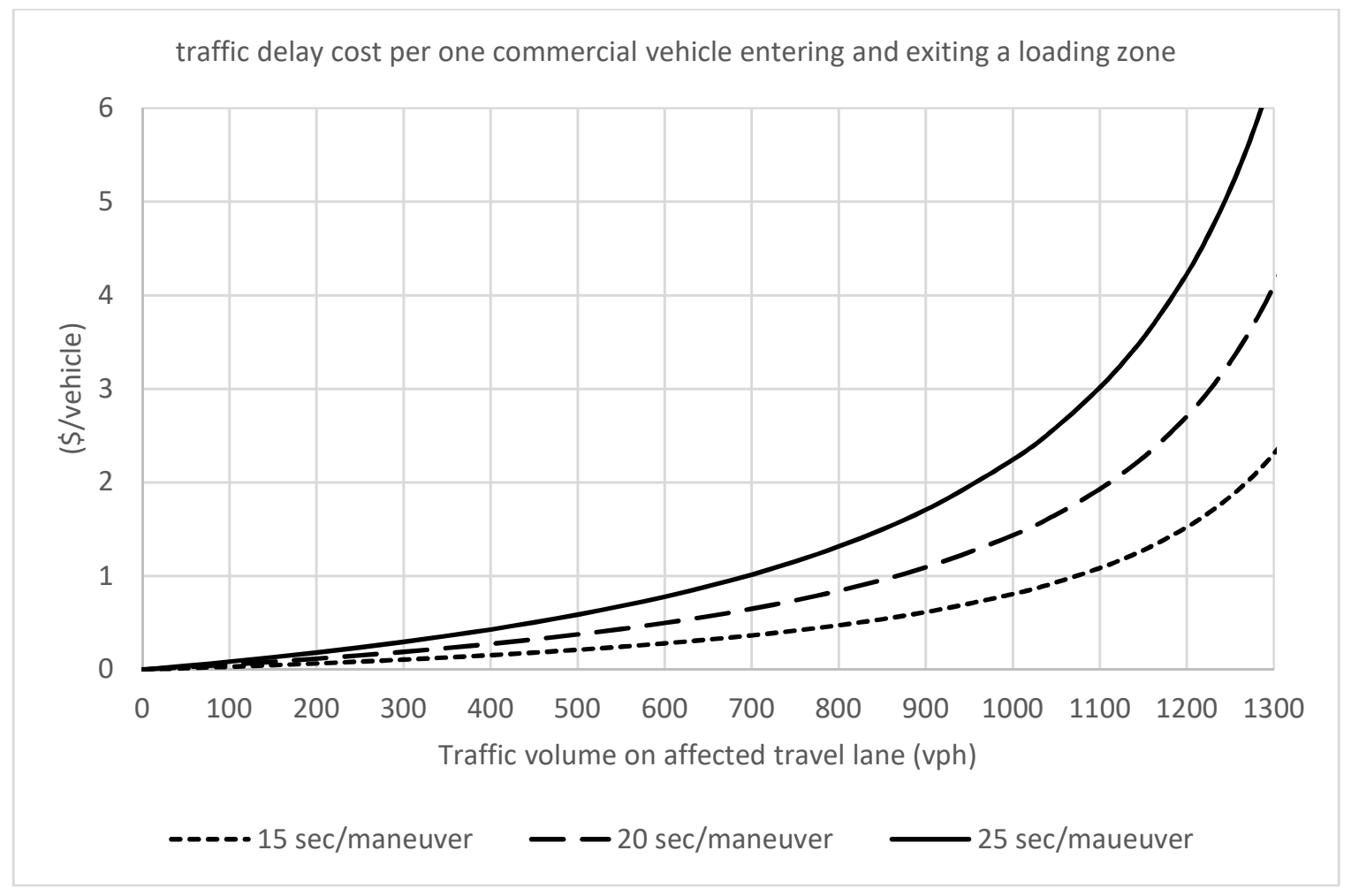

Figure 3.12 Traffic parking-maneuver delay cost of a commercial vehicle 
The corridor double-parking traffic delay cost is estimated using $E q(3.13)$. Based on Table 3.1, the corridor double-parking traffic delay cost $\left(c_{d b}\right)$ is computed, as shown in Figure 3.13. In addition, the number of vehicles delays by a double-parking commercial vehicle are calculated. The traffic delay cost per one vehicle $\left(f_{d b}\right)$ is computed, as shown in Figure 3.14. Since many double-parking commercial vehicles are couriers, the doubleparking duration of a commercial vehicle is assumed to be very short ( 3 minutes). If the traffic volume is 1,300 vehicles per hour, $c_{d b}$ and $f_{d b}$ will equal $7.16 \$$ /event and 0.006 hour/event/vehicle, respectively.

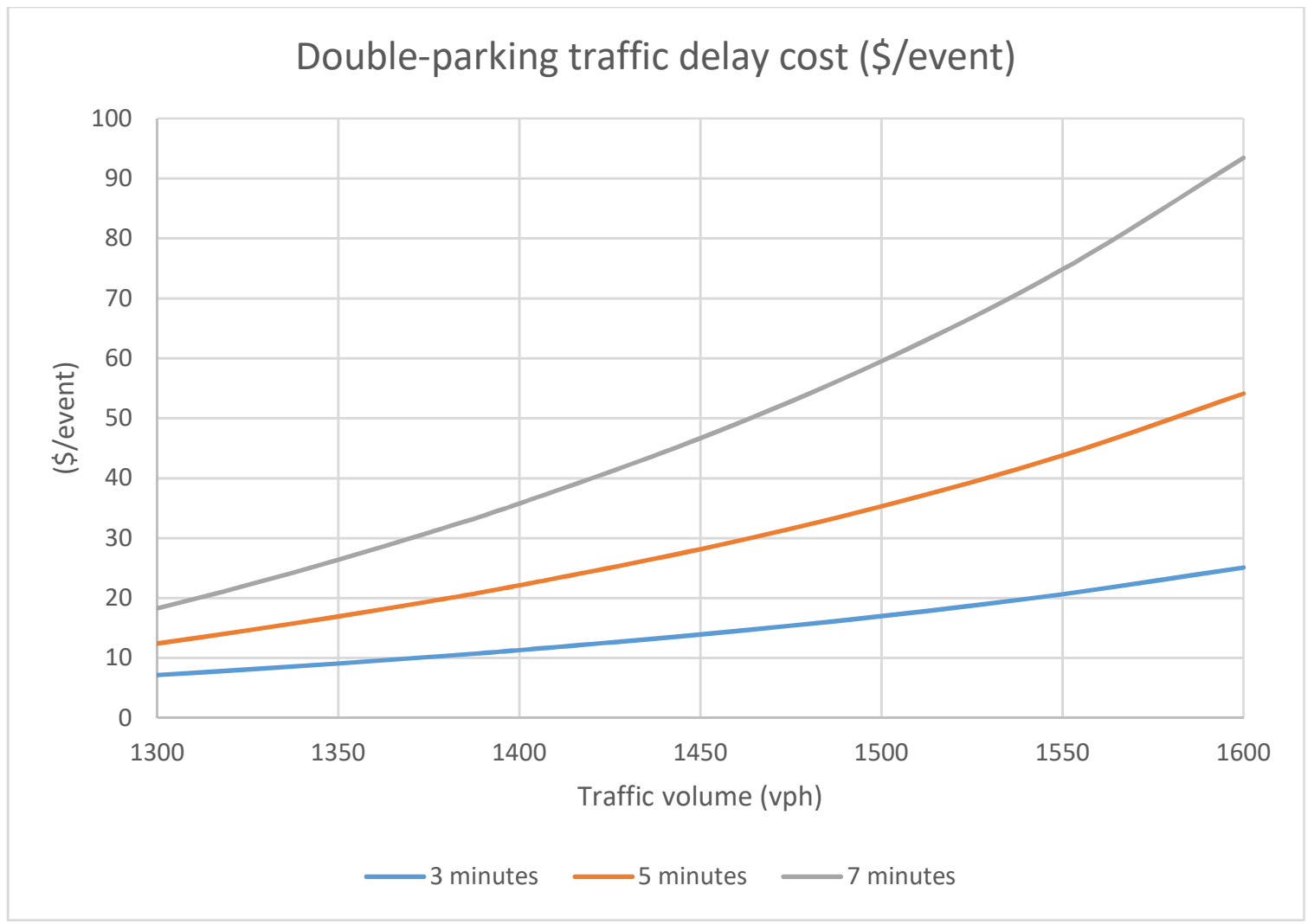

Figure 3.13 Corridor double-parking traffic delay cost 


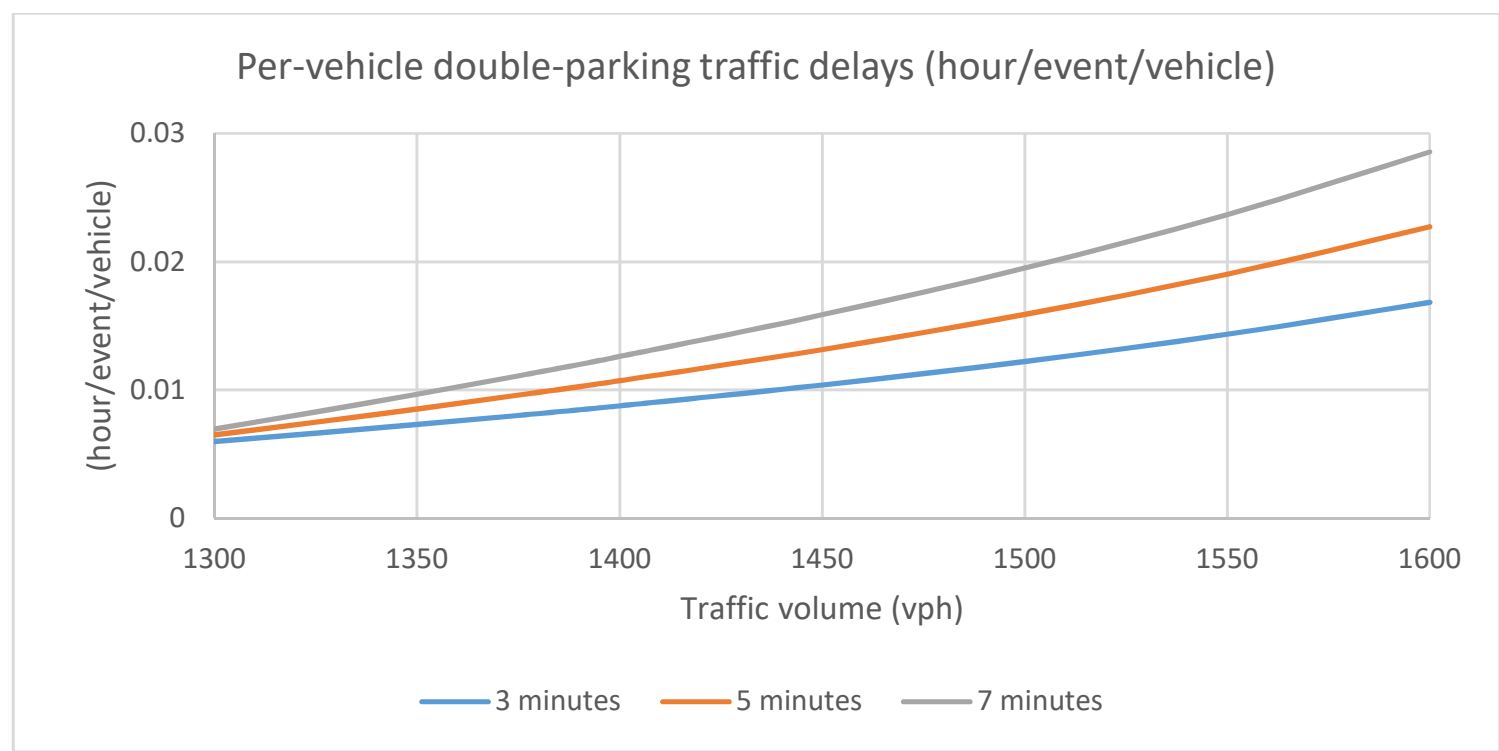

Figure 3.14 Double-parking traffic delays per car per one double-parking event

\subsection{Conclusion}

An on-street parking system is associated with traffic delays in several ways. Firstly, while vehicles enter or vacate parking spots, they temporarily block traffic lanes adjacent to the parking spots. Secondly, if commercial drivers double park in a traffic lane, the commercial vehicles will block the traffic lane while they serve customers. This chapter proposes how to estimate these traffic delays.

The double-parking event takes place in a downtown area and often occurs in late morning (Section 2.2), as that is prime time for commercial vehicles serving many establishments including restaurants and stores. Based on the case study (Section 3.7), the corridor double-parking traffic delay cost $\left(c_{d b}\right)$ equals $\$ 7.16$ per event and the per-vehicle traffic delays $\left(f_{d b}\right)$ equal 0.006 hour/event/vehicle. In addition, traffic delay cost due to parking maneuver of a passenger car $\left(c_{d p}\right)$ and a commercial vehicle $\left(c_{d c}\right)$ equals $\$ 0.13$ and $\$ 0.65$ per maneuver, respectively. 


\section{Chapter 4 Overview of On-Street Parking Models}

This chapter explains the overview of the on-street parking optimization models. The main sections are a description of an on-street parking system, objective function and decision variables, costs associated with the system, model constraints, the number of vehicles parking on-street and the number of vehicles parking in other alternatives, and scenarios for developing parking optimization models.

\subsection{Description of on-street parking system}

An on-street parking system in an urban area have two main components: parking spaces and loading zones. Parking spaces are designated for passenger cars. Loading zones are designated for commercial vehicles. When passenger cars park on-street, most of the vehicles park for short time periods (The Transportation Research Board, 1971, Shu-chun et al., 2008). Commercial vehicles usually park close to customers in order to ease delivery and service with short walking distances and low chance of theft.

In urban area, drivers have multiple parking options. Passenger car drivers have onstreet and off-street options, as shown in Figure 4.1. The on-street option means that a driver decides to park on street. Sometimes, drivers search for parking since all the parking spaces are full. The chosen on-street parking spaces might be far from final destinations; therefore, drivers have to walk. The off-street option includes parking garages and parking lots. Garages and lots are assumed to be close to final destinations so walking times are negligible. However, drivers will spend some time inside these garages and lots. Double parking is not an option for passenger cars because it is the least chosen option for passenger cars and many studies (Calthrop and Proost, 2006; Shoup, 2006; Arnott and 
Rowse, 2009; Zhou, 2014; Inci and Lindsey, 2015) assume that passenger vehicles parking on street and off street.

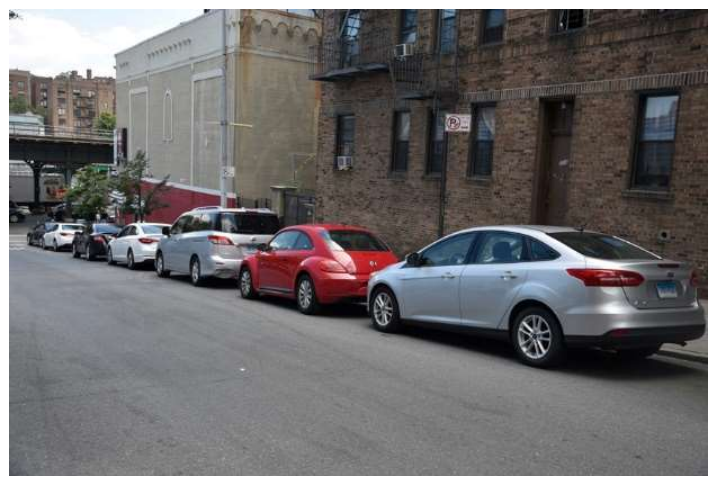

On street parking spaces

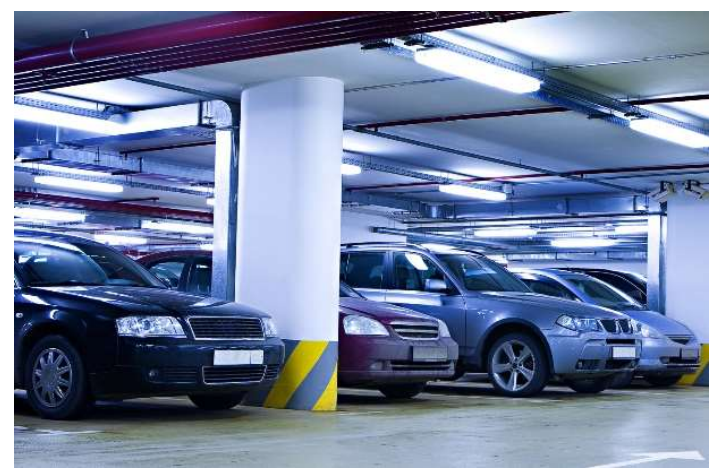

Off street garages

Figure 4.1 Parking options of passenger cars

Commercial vehicle drivers have on-street parking and double-parking options, as shown in Figure 4.2. The on-street option means that a commercial driver decides to park in a loading zone. Sometimes, all the loading zones are full. Therefore, a driver will search for parking until a loading zone becomes available. The double-parking option means that a commercial driver decides to park in a traffic lane and performs a pickup, delivery, or service. The commercial driver has risk receiving a double-parking ticket. Off-street parking is not an option for commercial vehicles because off-street parking locations are usually far from customers, resulting in long distances to handle goods and equipment and high chance of theft. 


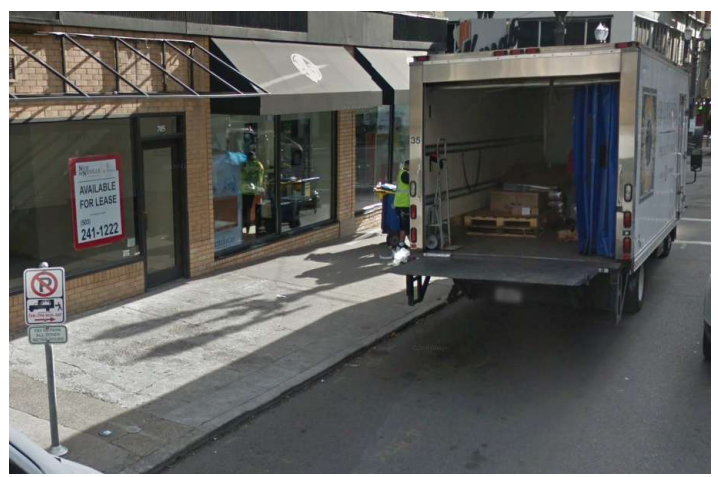

On street loading zones

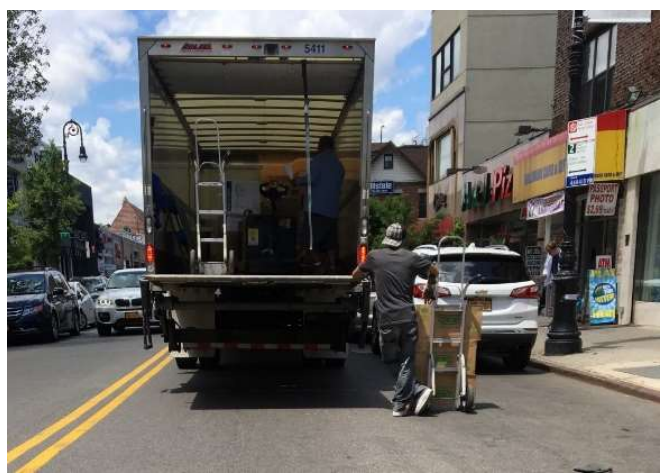

Double parking

Figure 4.2 Parking options of commercial vehicles

\subsection{Objective function and decision variable}

The on-street parking system is optimized to maximize parking revenues or to maximize parking revenues minus costs that is called "social optimum (SOC)". The parking revenues equal how much car drivers and commercial drivers pay to park on street. The costs include parking search costs and traffic delay costs. The costs are explained later in Section 4.3.

The decision variables are meter rate of loading zones $\left(c_{c o}\right)$, meter rate of parking spaces $\left(c_{p o}\right)$, the number of loading zones $\left(n_{c}\right)$, and the number of parking spaces $\left(n_{p}\right)$. The meter rates can be positive and negative. The positive values mean that drivers pay parking fees. The negative values mean that drivers receive incentive for parking on street instead of double parking. The number of parking types represent curb length allocation.

\subsection{Estimation of costs associated with parking}

The costs associated with parking are costs to parkers and traffic delay costs. For the on-street option, the total costs include monetary cost and parking search cost. The onstreet monetary cost is how much drivers actually pay to park on street e.g. parking fee. The parking search cost is associated with how long drivers spend time cruising for 
parking. For the double-parking option, the total cost is expected double-parking fine. For the off-street option, the total costs include monetary cost and expected parking search cost, which is assumed to be constant for garages and parking lots.

The traffic delay costs are caused by maneuver delays and double-parking delays. The maneuver delays occur when vehicles enter and exit parking spots on street. These maneuvers block traffic. The double-parking delays occur when commercial vehicles double park. These double-parking events block traffic, including through traffic and car drivers who search for on-street parking spaces.

\subsubsection{On-street monetary cost}

The on-street monetary cost is how much drivers actually pay to park in street. For each commercial driver, the monetary cost equals the commercial vehicle parking meter rate $\left(c_{c o}\right)$ multiplied by customer service time $\left(t_{s}\right)$. Since customer service time $\left(t_{s}\right)$ is an inverse of service rate of loading zones $\left(\mu_{c}\right)$, the on-street monetary cost to each commercial vehicle is expressed as follows,

$\frac{c_{c o}}{\mu_{c}}$

Similarly, for each passenger driver, the monetary cost equals the passenger car onstreet parking meter rate $\left(c_{p o}\right)$ multiplied by parking duration. Since parking duration is an inverse of service rate of parking spaces $\left(\mu_{p}\right)$, the on-street monetary cost to each passenger car is expressed as follows, $\frac{c_{p o}}{\mu_{p}}$ 
The on-street monetary cost equals parking revenue. Parking revenue is money that a parking authority collects from drivers to park on street. However, the on-street monetary cost is expense to drivers.

\subsubsection{Parking search cost}

Parking search cost is the cost of searching for on-street parking. The parking search cost equals the product of the value of time and the parking search time.

The parking search time has been estimated with multiple equations. Lam et al (2006) proposed an equation for calculating the searching time for a parking space. The equation is the Bureau of Public Roads (BPR) function. The ratio of demand to capacity is a parking utilization factor. Millard-Ball et al (2014) estimated the parking search time, based on the queuing theory. Zheng and Geroliminis (2016) estimated the parking search time based on the average cruising speed and the expected cruising distance that equals the space between two parking spaces divided by the probability of available parking spaces.

For this study, the parking search time is estimated based on Lam et al (2006)'s equation. A parking utilization factor is a ratio of the number of vehicles parking on street to the service rate of parking supply. For commercial vehicles, the number of vehicles parking on street is defined as commercial revenue demand $\left(\lambda_{c r}\right)$ that will be explained in Section 4.5. The service rate of loading zones is the number of loading zones $\left(n_{c}\right)$ multiplied by the service rate of one loading zone $\left(\mu_{c}\right)$. The parking search time of a commercial vehicle is expressed as follows,

$\alpha_{w c}\left(\frac{\lambda_{c}}{n_{c} \mu_{c}}\right)^{\gamma_{c}}$ 
Both $\alpha_{w c}$ and $\gamma_{c}$ are parameters. The value of time of commercial vehicles is $c_{c t}$. The parking search cost is expressed below,

$c_{c t} \alpha_{w c}\left(\frac{\lambda_{c}}{n_{c} \mu_{c}}\right)^{\gamma_{c}}$

For passenger cars, the number of cars parking on street is defined as passenger car revenue demand $\left(\lambda_{p r}\right)$ that will be explained in Section 4.5. The service rate of parking spaces is the number of parking spaces $\left(n_{p}\right)$ multiplied by the service rate of one parking space $\left(\mu_{p}\right)$. The expected parking search time of a parking car is expressed below, $\alpha_{w p}\left(\frac{\lambda_{p}}{n_{p} \mu_{p}}\right)^{\gamma_{p}}$

Both $\alpha_{w p}$ and $\gamma_{p}$ are parameters.

This estimation is reasonable only when the parking demand is lower than the parking supply. In other words, the parking utilization factor has to be less than 1.0 (Cheng, et al., 2016)

Loading zones are often in front of destinations of commercial vehicles, which are establishments. However, parking spaces are not necessarily in front of destinations. Car drivers usually walk from parking locations to destinations. A ratio of the walking duration to parking search delays, which is defined as $\vartheta$, is assumed to be fixed. The parking search time plus the walking time equals, $(1+\vartheta) \alpha_{w p}\left(\frac{\lambda_{p}}{n_{p} \mu_{p}}\right)^{\gamma_{p}}$

The value of time of passenger cars is $c_{p t}$. The parking search cost is expressed below, 
$c_{p t}(1+\vartheta) \alpha_{w p}\left(\frac{\lambda_{p}}{n_{p} \mu_{p}}\right)^{\gamma_{p}}$

\subsubsection{Double parking fine}

This cost will occur when commercial vehicles double park and a commercial driver has risk receiving a double-parking ticket. Therefore, it equals a double-parking fine $\left(c_{v}\right)$ multiplied by the probability of receiving a double-parking ticket. The probability equals service time divided by parking enforcement cycle duration. The service time is an inverse of service rate of loading zones $\left(\mu_{c}\right)$. The expected enforcement cycle duration is the enforcement cycle duration of each officer $\left(\tau_{e}\right)$ divided by the number of enforcement officers $\left(n_{e}\right)$. The double-parking cost is expressed as follow,

$\frac{c_{v} n_{e}}{\mu_{c} \tau_{e}}$

\section{$\underline{\text { 4.3.4 Off-street parking cost }}$}

The off-street parking cost includes the fixed parking search cost $\left(c_{p f f}\right)$ and offstreet monetary cost. The off-street monetary cost equals a meter rate $\left(c_{p f}\right)$ multiplied by parking duration. The parking duration is an inverse of service rate of parking spaces $\left(\mu_{p}\right)$. The total off-street cost is expressed as follows, $\frac{c_{p f}}{\mu_{p}}+c_{p f f}$

\section{$\underline{4.3 .5 \text { Maneuver traffic delay cost }}$}

Maneuver traffic delays occurs while a vehicle enters or exits an on-street parking spot. The maneuver traffic delay costs of both commercial vehicles and cars are explained in more details and estimated in Chapter 3. 


\subsubsection{Double parking traffic delay cost}

Double parking delays occur when a commercial vehicle parks in a traffic lane and a commercial driver performs a pickup, delivery, or service. When a commercial vehicle double parks, it blocks the traffic lane that creates a bottleneck and leads to queue and delays to through traffic. The double-parking traffic delay cost is explained in more details and estimated in Chapter 3.

\subsubsection{Increased travel cost of a parking passenger car}

When a commercial vehicle double parks, it creates a queue. A parking car might be behind and close to the double-parking commercial vehicle. The parking car has to get through the queue, which increases its travel time.

Double-parking events occur when the arrival rate of commercial vehicles $\left(\lambda_{c}\right)$ is greater than the commercial revenue demand $\left(\lambda_{c r}\right)$. The number of double-parking events in one hour is $\left(\lambda_{c}-\lambda_{c r}\right) . t_{s}$ is defined as an average parking search time of passenger cars. The expected number of double-parking events that are experienced by a parking car is $t_{s}\left(\lambda_{c}-\lambda_{c r}\right)$. The expected double-parking delays to one vehicle is $f_{d b}$. Therefore, the increased travel time of a parking car is,

$f_{d b} t_{s}\left(\lambda_{c}-\lambda_{c r}\right)$

The increase in travel cost of a parking passenger car is expressed below, $c_{p t} f_{d b} t_{s}\left(\lambda_{c}-\lambda_{c r}\right)$

The parking car's travel time is increased only when commercial vehicles double park. The on-street parking cost of passenger cars is affected by loading zones. This effect is called "jointed". However, if commercial vehicles do not double park, the on-street 
parking cost of passenger cars will not be affected by loading zones. This case is called "non-jointed".

\subsection{Model constraint}

The model constraint is on curb length. The number of loading zones $\left(n_{c}\right)$ and parking spaces $\left(n_{p}\right)$ is limited by available curb length. Total length of loading zones $\left(n_{c}\right.$ multiplied by unit length of a loading zone $\left.l_{c}\right)$ plus total length of parking spaces $\left(n_{p}\right.$ multiplied by unit length of a parking space $l_{p}$ ) is not greater than available curb length $\left(l_{b}\right)$. In addition, $n_{c}$ and $n_{p}$ are numbers of parking spaces so they have to be positive. The constraints on $n_{c}$ and $n_{p}$ are shown below,

$$
\begin{aligned}
& n_{c} l_{c}+n_{p} l_{p} \leq l_{b} \\
& n_{c} \geq 0 \\
& n_{p} \geq 0
\end{aligned}
$$

Since the model objectives are to maximize revenue and social optimum value, all the available parking length are allocated to both parking types. In other words, the constraint on total parking length is binding. Then, the constraints become,

$$
\begin{aligned}
& n_{c} l_{c}+n_{p} l_{p}=l_{b} \\
& n_{c} \geq 0 \\
& n_{p} \geq 0
\end{aligned}
$$

The constraints above can be expressed in another form, as shown below,

$$
\begin{aligned}
& n_{p}=\frac{\left(l_{b}-n_{c} l_{c}\right)}{l_{p}} \\
& n_{c} \geq 0 \\
& n_{c} l_{c} \leq l_{b}
\end{aligned}
$$


The nonnegativity constraint can be relaxed. After optimizing $n_{c}$, its values can be checked if they are not negative. The equality constraint shows that $n_{c}$ is a function of $n_{p}$. This constraint can be put in the objective function so the objective function is no longer a function of $n_{c}$. Then the constraint become $n_{p} l_{p} \leq l_{b}$.

\subsection{Number of vehicles parking on street}

From section 4.1, drivers have multiple parking options: on-street parking and the other option. This section estimates how many commercial vehicles and passenger cars park on street. The first step is to calculate total costs of available options. The second step is to estimate the number of vehicles parking on street, which is called revenue demand.

\subsubsection{Costs of commercial vehicle parking options}

The cost of a commercial vehicle parking on street includes the monetary cost and the parking delay cost. The estimation of the costs is explained in Section 4.3. The total on-street cost can be expressed as follow,

$\frac{c_{c o}}{\mu_{c}}+c_{c t} \alpha_{w c}\left(\frac{\lambda_{c r}}{n_{c} \mu_{c}}\right)^{\gamma_{c}}$

The cost of a commercial vehicle double parking equals the expected doubleparking fine is expressed as follow,

$\frac{c_{v} n_{e}}{\mu_{c} \tau_{e}}$

\subsubsection{Commercial breakeven parking demand}

The on-street cost depends on the commercial revenue demand $\left(\lambda_{c r}\right)$. The more the $\lambda_{c r}$, the higher the cost. The double-parking cost does not depend on $\lambda_{c r}$. The two costs will be equal for a certain value of $\lambda_{c r}$, which is called commercial breakeven demand 
$\left(\lambda_{c}^{*}\right)$. No more drivers park on street; otherwise, the on-street cost will be higher than the double-parking cost. This is an equilibrium state, (Shoup, 2006), which is expressed below,

$$
\begin{aligned}
& \frac{c_{c o}}{\mu_{c}}+c_{c t} \alpha_{w c}\left(\frac{\lambda_{c}^{*}}{n_{c} \mu_{c}}\right)^{\gamma_{c}}=\frac{c_{v} n_{e}}{\mu_{c} \tau_{e}} \\
& c_{c t} \alpha_{w c}\left(\frac{\lambda_{c}^{*}}{n_{c} \mu_{c}}\right)^{\gamma_{c}}=\frac{c_{v} n_{e}}{\mu_{c} \tau_{e}}-\frac{c_{c o}}{\mu_{c}} \\
& \left(\frac{\lambda_{c}^{*}}{n_{c} \mu_{c}}\right)^{\gamma_{c}}=\frac{c_{v} n_{e}-c_{c o} \tau_{e}}{c_{c t} \alpha_{w c} \mu_{c} \tau_{e}} \\
& \lambda_{c}^{*}=n_{c} \mu_{c}\left(\frac{c_{v} n_{e}-c_{c o} \tau_{e}}{c_{c t} \alpha_{w c} \mu_{c} \tau_{e}}\right)^{\frac{1}{\gamma_{c}}}
\end{aligned}
$$

If the arrival rate of commercial vehicles $\left(\lambda_{c}\right)$ is not greater than $\lambda_{c}^{*}$, all the commercial vehicles will park on street. $\lambda_{c r}$ equals $\lambda_{c}$. If $\lambda_{c}$ is greater than $\lambda_{c}^{*}$, some commercial vehicles will double park. $\lambda_{c r}$ equals $\lambda_{c}^{*}$. The number of double-parking events equals $\left(\lambda_{c}-\lambda_{c}^{*}\right)$.

\subsubsection{Costs of passenger vehicle parking options}

The cost of a passenger vehicle parking on street includes the on-street monetary cost, the parking delay cost, and an increase in travel cost due to double-parking events. The estimation of the costs is explained in Section 4.3. The on-street cost can be expressed as follow,

$$
\frac{c_{p o}}{\mu_{p}}+c_{p t}(1+\vartheta) \alpha_{w p}\left(\frac{\lambda_{p}}{n_{p} \mu_{p}}\right)^{\gamma_{p}}+c_{p t} f_{d b} t_{s}\left(\lambda_{c}-\lambda_{c r}\right)
$$

From the expression above, the last term is associated with loading zones. If some commercial vehicles double park $\left(\lambda_{c}-\lambda_{c r}>0\right)$, car drivers will be delayed by doubleparking events during parking search. The on-street cost is affected by loading zones. This 
is a joint $(\mathrm{J})$ effect. If commercial vehicles do not double park $\left(\lambda_{c}-\lambda_{c r}=0\right)$, car drivers will not be delayed by double-parking events. This case is called "non-joint" (NJ).

The cost of a passenger car parking off street includes off-street monetary cost and fixed parking search cost. The estimation of the costs is explained in Section 4.3. The cost can be expressed as follow,

$\frac{c_{p f}}{\mu_{p}}+c_{p f f}$

\subsubsection{Breakeven passenger car parking demand}

The on-street cost depends on the car revenue demand $\left(\lambda_{p r}\right)$. The more the $\lambda_{p r}$, the higher the cost. The off-street cost does not depend on $\lambda_{p r}$. The two costs will be equal for a certain value of $\lambda_{p r}$, which is called passenger car breakeven demand $\left(\lambda_{p}^{*}\right)$. No more drivers park on street; otherwise, the on-street cost will be higher than the off-street cost. This is an equilibrium state, (Shoup, 2006). $\lambda_{p}^{*}$ is calculated in 2 cases: joint (with doubleparking events) and non-joint (without double-parking events).

Joint (with double-parking events)

$$
\begin{aligned}
& \frac{c_{p o}}{\mu_{p}}+c_{p t}(1+\vartheta) \alpha_{w p}\left(\frac{\lambda_{p}^{*}}{n_{p} \mu_{p}}\right)^{\gamma_{p}}+c_{p t} f_{d b} t_{s}\left(\lambda_{c}-\lambda_{c}^{*}\right)=\frac{c_{p f}}{\mu_{p}}+c_{p f f} \quad E q(4.3) \\
& c_{p t}(1+\vartheta) \alpha_{w p}\left(\frac{\lambda_{p}^{*}}{n_{p} \mu_{p}}\right)^{\gamma_{p}}=\frac{c_{p f}}{\mu_{p}}+c_{p f f}-\frac{c_{p o}}{\mu_{p}}-c_{p t} f_{d b} t_{s}\left(\lambda_{c}-\lambda_{c}^{*}\right) \\
& \left(\frac{\lambda_{p}^{*}}{n_{p} \mu_{p}}\right)^{\gamma_{p}}=\frac{\frac{c_{p f}}{\mu_{p}}+c_{p f f}-\frac{c_{p o}}{\mu_{p}}-c_{p t} f_{d b} t_{s}\left(\lambda_{c}-\lambda_{c}^{*}\right)}{c_{p t}(1+\vartheta) \alpha_{w p}} \\
& \lambda_{p}^{*}(J)=n_{p} \mu_{p}\left[\frac{c_{p f}}{c_{p t}(1+\vartheta) \alpha_{w p}} E q(4.4)\right. \\
& c_{p f f}-\frac{c_{p o}}{\mu_{p}}-c_{p t} f_{d b} t_{s}\left(\lambda_{c}-\lambda_{c}^{*}\right) \\
& \frac{1}{\gamma_{p}}
\end{aligned}
$$


From Section 4.5.2, $\quad \lambda_{c}^{*}=n_{c} \mu_{c}\left(\frac{c_{v} n_{e}-c_{c o} \tau_{e}}{c_{c t} \alpha_{w c} \mu_{c} \tau_{e}}\right)^{\frac{1}{\gamma_{c}}}$

$\lambda_{p}^{*}(J)=n_{p} \mu_{p}\left[\frac{\frac{c_{p f}}{\mu_{p}}+c_{p f f}-\frac{c_{p o}}{\mu_{p}}-c_{p t} f_{d b} t_{s}\left\{\lambda_{c}-n_{c} \mu_{c}\left(\frac{c_{v} n_{e}-c_{c o} \tau_{e}}{c_{c t} \alpha_{w c} \mu_{c} \tau_{e}}\right)^{\frac{1}{\gamma_{c}}}\right\}}{c_{p t}(1+\vartheta) \alpha_{w p}}\right]^{\frac{1}{\gamma_{p}}}$

Non-joint (without double-parking events)

$$
\begin{aligned}
& \frac{c_{p o}}{\mu_{p}}+c_{p t}(1+\vartheta) \alpha_{w p}\left(\frac{\lambda_{p}^{*}}{n_{p} \mu_{p}}\right)^{\gamma_{p}}=\frac{c_{p f}}{\mu_{p}}+c_{p f f} \\
& c_{p t}(1+\vartheta) \alpha_{w p}\left(\frac{\lambda_{p}^{*}}{n_{p} \mu_{p}}\right)^{\gamma_{p}}=\frac{c_{p f}}{\mu_{p}}+c_{p f f}-\frac{c_{p o}}{\mu_{p}} \\
& \left(\frac{\lambda_{p}^{*}}{n_{p} \mu_{p}}\right)^{\gamma_{p}}=\frac{\frac{c_{p f}}{\mu_{p}}+c_{p f f}-\frac{c_{p o}}{\mu_{p t}}}{c_{p t}(1+\vartheta) \alpha_{w p}} \\
& \lambda_{p}^{*}(N J)=n_{p} \mu_{p}\left[\frac{c_{p f}+c_{p f} \mu_{p}-c_{p o}}{c_{p t}(1+\vartheta) \alpha_{w p} \mu_{p}}\right]^{\frac{1}{\gamma_{p}}}
\end{aligned}
$$

The car breakeven parking demand is estimated in two cases: with and without double-parking events, as shown with Figure 4.3.

If the arrival rate of passenger cars $\left(\lambda_{p}\right)$ is not greater than $\lambda_{p}^{*}$, all the cars will park on street. $\lambda_{p r}$ equals $\lambda_{p}$. If $\lambda_{p}$ is greater than $\lambda_{p}^{*}$, some cars will park off street. $\lambda_{p r}$ equals $\lambda_{p}^{*}$. The number of cars parking off street equals $\left(\lambda_{p}-\lambda_{p}^{*}\right)$. 


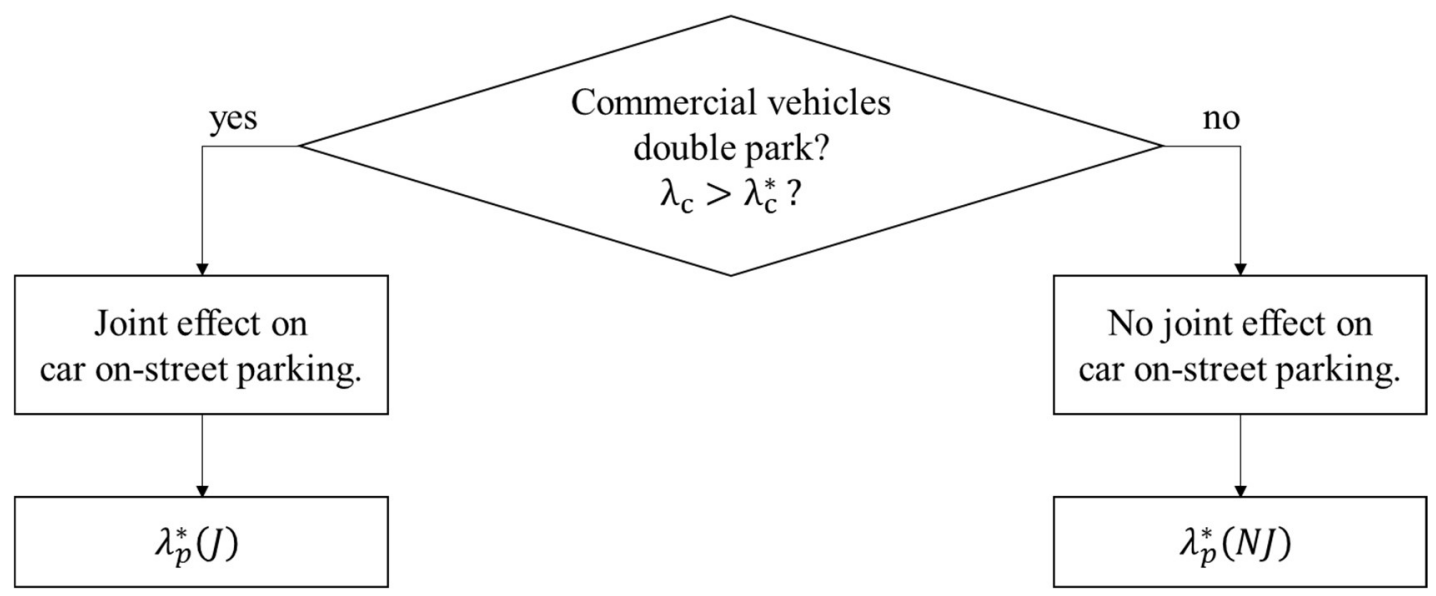

Figure 4.3 Joint effect on car breakeven demand

\subsubsection{Summary of breakeven demand and interpretation}

From Section 4.5.2 and Section 4.5.4, the breakeven demand is shown below, Commercial breakeven demand

$\lambda_{c}^{*}=n_{c} \mu_{c}\left(\frac{c_{v} n_{e}-c_{c o} \tau_{e}}{c_{c t} \alpha_{w c} \mu_{c} \tau_{e}}\right)^{\frac{1}{\gamma_{c}}}$

Car breakeven demand

$\lambda_{p}^{*}(J)=n_{p} \mu_{p}\left[\frac{\frac{c_{p f}}{\mu_{p}}+c_{p f f}-\frac{c_{p o}}{\mu_{p}}-c_{p t} f_{d b} t_{s}\left(\lambda_{c}-\lambda_{c}^{*}\right)}{c_{p t}(1+\vartheta) \alpha_{w p}}\right]^{\frac{1}{\gamma_{p}}}$

$\lambda_{p}^{*}(N J)=n_{p} \mu_{p}\left[\frac{c_{p f}+c_{p f f} \mu_{p}-c_{p o}}{c_{p t}(1+\vartheta) \alpha_{w p} \mu_{p}}\right]^{\frac{1}{\gamma_{p}}}$

There are two estimates for the car breakeven demand. The first one has a jointed impact where some commercial vehicles double park. A double-parking vehicle blocks one traffic lane, which increases delays to traffic and parking cars. The second one does not have a jointed impact since no commercial vehicles double park. 


\section{$\underline{\text { 4.5.6 Passenger car parking equivalent }}$}

The passenger car parking equivalent reflects how a commercial vehicle uses a parking space, compared with a passenger car, in terms of unit parking length and parking duration. The passenger car parking equivalent can be formulated below, $\left(\frac{l_{c}}{l_{p}}\right)\left(\frac{\mu_{p}}{\mu_{c}}\right)$

The aggregated arrival rate of equivalent passenger cars $\left(\lambda_{a}\right)$ is the arrival rate of both commercial vehicles and passenger cars, compared with a passenger car in terms of unit parking length and parking duration, which is expressed below,

$$
\lambda_{a}=\lambda_{c} \frac{l_{c}}{l_{p}} \frac{\mu_{p}}{\mu_{c}}+\lambda_{p}
$$

The number of equivalent parking spaces $\left(n_{a}\right)$ can be expressed below,

$n_{a}=\frac{l_{b}}{l_{p}}$

The equivalent parking search cost can be expressed below,

$c_{p t}(1+\vartheta) \alpha_{w p}\left(\frac{\lambda_{a}}{n_{a} \mu_{p}}\right)^{\gamma_{p}}$

With the assumption of no commercial vehicles double parking, the equivalent breakeven demand can be expressed below,

$\lambda_{a}^{*}(N J)=n_{a} \mu_{p}\left[\frac{c_{p f}+c_{p f f} \mu_{p}-c_{p o}}{c_{p t}(1+\vartheta) \alpha_{w p} \mu_{p}}\right]^{\frac{1}{\gamma_{p}}}$

\subsection{Scenarios for developing parking models}

From Section 4.5, the commercial revenue demand $\left(\lambda_{c r}\right)$ equals the minimum value of the arrival rate of commercial vehicles $\left(\lambda_{c}\right)$ and the commercial breakeven demand $\left(\lambda_{c}^{*}\right)$. 
Similarly, the passenger car revenue demand $\left(\lambda_{p r}\right)$ is the minimum value of the arrival rate of cars $\left(\lambda_{p}\right)$ and the car breakeven demand $\left(\lambda_{p}^{*}\right)$. This results in variation in estimates of the revenue demand. A combination of different estimates is created and called scenarios for developing parking models in Chapters 5 and 7, as shown in Table 4.1. Based on the variation in revenue demand, there are four scenarios to optimize.

Table 4.1 Parking management strategy and scenarios for parking models

\begin{tabular}{|c|c|c|c|c|}
\hline Value of $\lambda_{c}$ & Estimate for $\lambda_{c r}$ & Value of $\lambda_{p}$ & Estimate for $\lambda_{p r}$ & Scenario \\
\hline$\lambda_{c} \leq \lambda_{c}^{*}$ & $\lambda_{c r}=\lambda_{c}$ & $\lambda_{p} \leq \lambda_{p}^{*}(N J)$ & $\lambda_{p r}=\lambda_{p}$ & 1 \\
\cline { 3 - 5 } & & $\lambda_{p}>\lambda_{p}^{*}(N J)$ & $\lambda_{p r}=\lambda_{p}^{*}(N J)$ & 2 \\
\hline$\lambda_{c}>\lambda_{c}^{*}$ & $\lambda_{c r}=\lambda_{c}^{*}$ & $\lambda_{p} \leq \lambda_{p}^{*}(J)$ & $\lambda_{p r}=\lambda_{p}$ & 3 \\
\cline { 3 - 5 } & & $\lambda_{p}>\lambda_{p}^{*}(J)$ & $\lambda_{p r}=\lambda_{p}^{*}(J)$ & 4 \\
\hline
\end{tabular}

Scenario 1 represents all vehicles parking on street, as shown in Figure 4.4. The assumption is that arrival rates of vehicles are lower than their breakeven demand

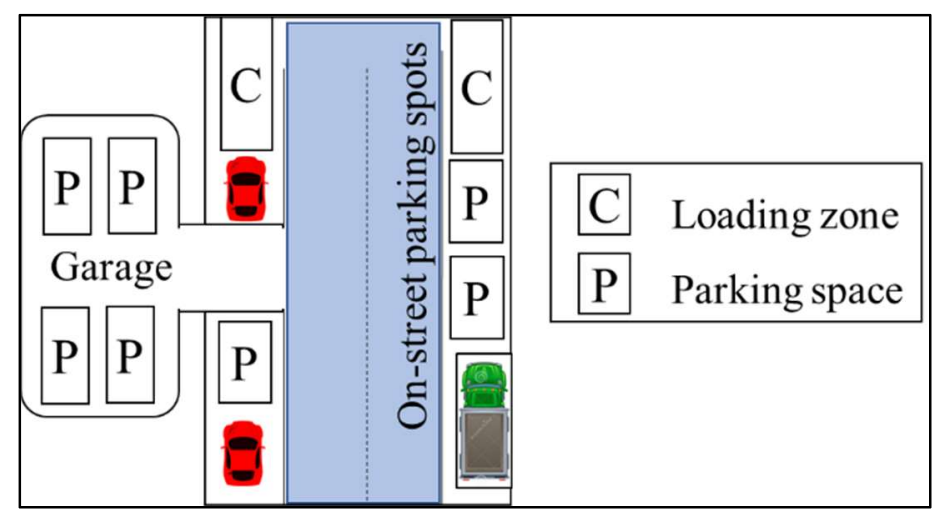

Figure 4.4 Scenario 1: All vehicles parking on street 
Scenario 2 represents commercial vehicles parking on street while passenger cars parking on street and off street, as shown in Figure 4.5. The assumption is that, of commercial vehicles, the arrival rate is lower than the breakeven demand. Of passenger cars, the arrival rate is higher than the breakeven demand.

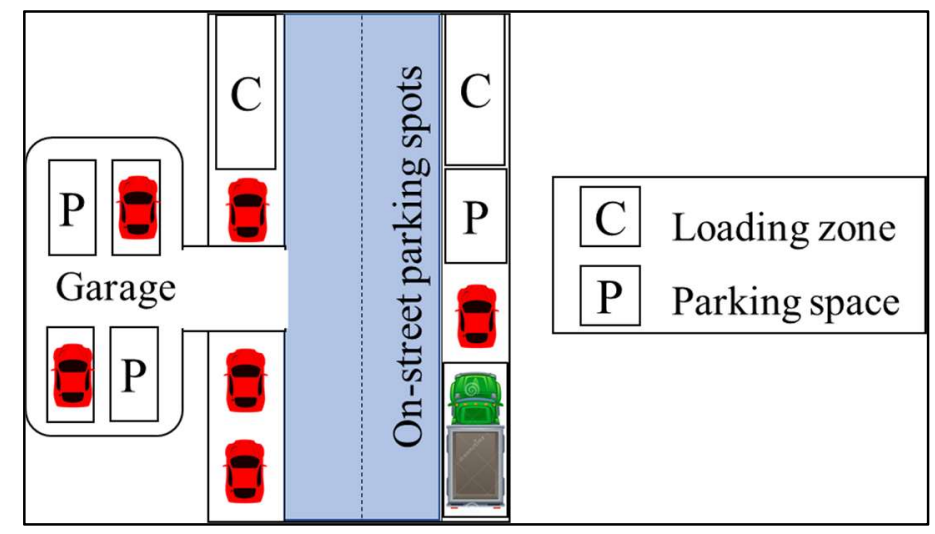

Figure 4.5 Scenario 2: Some passenger cars parking off street

Scenario 3 represents commercial vehicles parking on street and double parking while passenger cars parking on street, as shown in Figure 4.6. The assumption is that, of commercial vehicles, the arrival rate is higher than the breakeven demand. Of passenger cars, the arrival rate is lower than the breakeven demand.

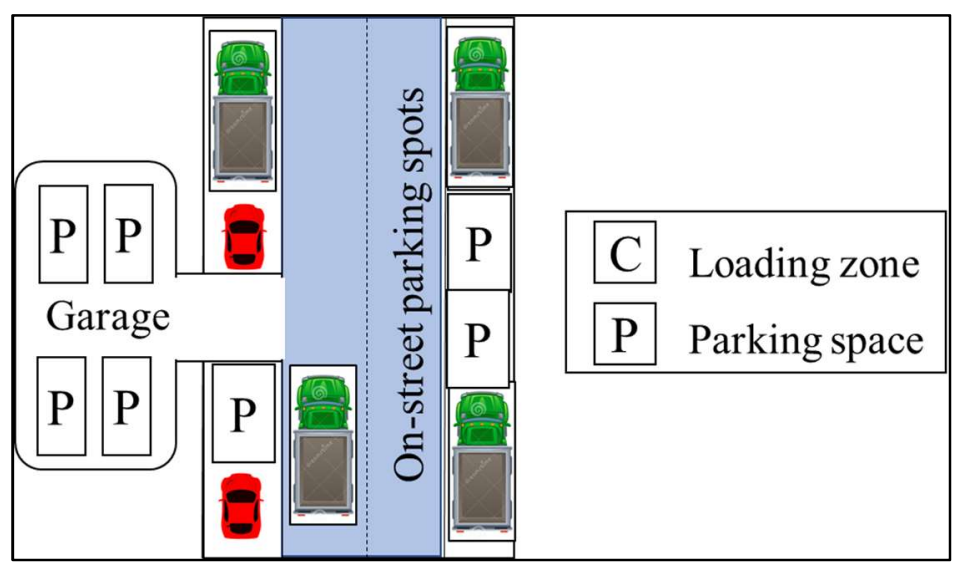

Figure 4.6 Scenario 3: Some commercial vehicles double parking 
Scenario 4 represents all the parking options are chosen, as shown in Figure 4.7. The assumption is that the arrival rates are higher than their breakeven demand.

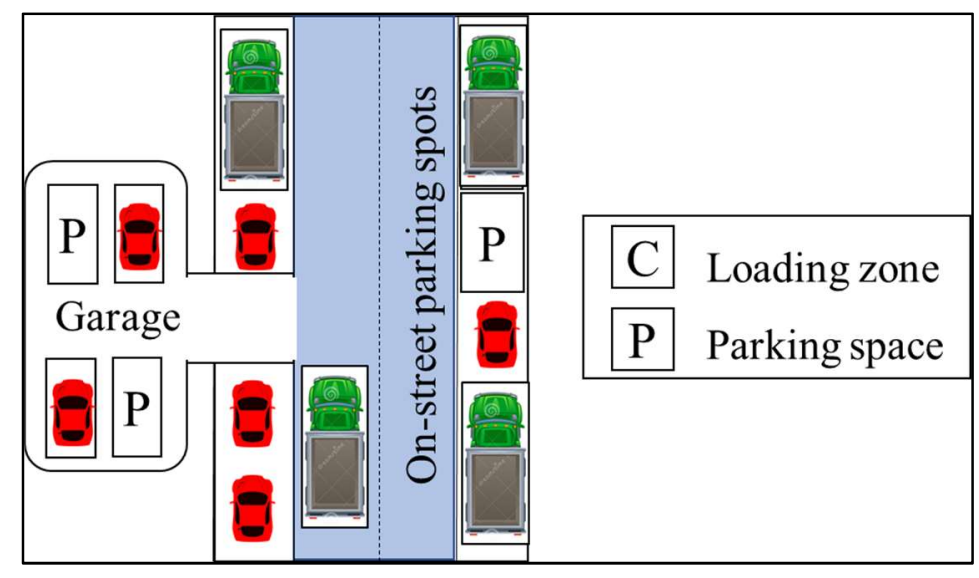

Figure 4.7 Scenario 4: All parking options are chosen

\subsection{Conclusion}

This chapter provides basis for the parking optimization models that are explained later in Chapters 5 and 7. The basis includes system description, objective functions and decision variables, estimates of costs associated with the on-street parking system, model constraints, number of vehicles parking on street, and scenarios for developing parking models. The objective functions are to maximize revenue and social optimum. The decision variables are curb length allocation $\left(n_{p}\right.$ and $\left.n_{c}\right)$ and meter rates $\left(c_{p o}\right.$ and $\left.c_{c o}\right)$. The cost estimates include monetary cost, parking search cost, double parking fine, off-street parking cost, maneuver traffic delay cost, double-parking traffic delay cost, and increased travel cost of a parking passenger car. The constraints are non-negativity on curb length allocation. Based on variation in the number of chosen parking options, four scenarios are set as basis for developing optimization models in Chapters 5 and 7 .

The next chapters propose how to optimize the on-street parking system, based on various objective functions and decision variables. Case studies are also included later. 


\section{Chapter 5 Revenue Optimization Models}

This chapter proposes a model maximizing total revenues from commercial vehicle loading zones and passenger car parking spaces. The main sections of this chapter are definitions of revenue optimization models, model formulation, optimal solutions for the four scenarios which is explained in Section 4.6, and solution interpretation.

\subsection{Definition of revenue optimization models}

The revenue optimization models are to maximize total revenues from both loading zones and parking spaces by optimizing decision variables. The decision variables are the

meter rate of loading zones $\left(c_{c o}\right)$, the meter rate of parking spaces $\left(c_{p o}\right)$, and the number of parking spaces $\left(n_{p}\right)$. The number of loading zones $\left(n_{c}\right)$ is calculated using the relationship between $n_{c}$ and $n_{p}$, as explained in Section 4.4.

\subsection{Model formulation}

Parking revenue includes revenue from commercial vehicles parking on street and cars parking on street. The objective function is formulated in this section. The constraints of the revenue models are explained in Section 4.3.

\subsubsection{Revenue from commercial vehicle parking}

From Section 4.3, parking revenue from a commercial vehicle parking equals the commercial vehicle parking meter rate $\left(c_{c o}\right)$ multiplied by the commercial revenue demand $\left(\lambda_{c r}\right)$ divided by service rate of loading zones $\left(\mu_{c}\right)$. The parking revenue from commercial vehicles parking on street is expressed as follows,

$\frac{c_{c o} \lambda_{c r}}{\mu_{c}}$ 


\subsubsection{Revenue from passenger vehicle parking}

Similar to commercial vehicles, from Section 4.3, parking revenue from a passenger vehicle parking equals the passenger vehicle on-street parking meter rate $\left(c_{p o}\right)$ multiplied by the car revenue demand $\left(\lambda_{p r}\right)$ divided by service rate of parking spaces $\left(\mu_{c}\right)$. The parking revenue from passenger vehicles parking on street is expressed as follows, $\frac{c_{p o} \lambda_{p r}}{\mu_{p}}$

\section{$\underline{\text { 5.2.3 Formulation of objective function }}$}

The objective of the model is to maximize parking revenue from both commercial vehicles and passenger cars parking on street, which is expressed as follows,

$y_{r e v}=\frac{c_{c o} \lambda_{c r}}{\mu_{c}}+\frac{c_{p o} \lambda_{p r}}{\mu_{p}}$

\subsection{Revenue model for Scenario 1: All vehicles parking on street}

Of the model for Scenario 1, the revenue optimization begins with constraints on the decision variables. The next step is to take the objective function into account to find the optimal solutions.

\subsubsection{Constraints on commercial vehicle parking}

All commercial vehicles park on street because the cost of on-street parking is not greater than the cost of off-street parking, which is expressed below,

$\frac{c_{c o}}{\mu_{c}}+c_{c t} \alpha_{w c}\left(\frac{\lambda_{c r}}{n_{c} \mu_{c}}\right)^{\gamma_{c}} \leq \frac{c_{v} n_{e}}{\mu_{c} \tau_{e}}$

$E q(5.2)$ is rearranged and expressed below,

$c_{c o} \leq \frac{c_{v} n_{e}}{\tau_{e}}-c_{c t} \alpha_{w c} \mu_{c}\left(\frac{\lambda_{c}}{n_{c} \mu_{c}}\right)^{\gamma_{c}}$ 
The expression above gives the maximum value of $c_{c o}$.

$E q(5.2)$ is rearranged and expressed below,

$n_{c} \geq\left(\frac{\lambda_{c}}{\mu_{c}}\right)\left(\frac{c_{c t} \alpha_{w c} \mu_{c}}{\frac{c_{v} n_{e}}{\tau_{e}}-c_{c o}}\right)^{\frac{1}{\gamma_{c}}}$

The expression above gives the minimum value of $n_{c}$.

\subsubsection{Constraints on passenger car parking}

All passenger cars park on street because the cost of on-street parking is not greater than the cost of off-street parking, which is expressed below,

$\frac{c_{p o}}{\mu_{p}}+c_{p t}(1+\vartheta) \alpha_{w p}\left(\frac{\lambda_{p}}{n_{p} \mu_{p}}\right)^{\gamma_{p}} \leq \frac{c_{p f}}{\mu_{p}}+c_{p f f} \quad E q(5.5)$

$E q(5.5)$ is rearranged and expressed below,

$c_{p o} \leq c_{p f}+c_{p f f} \mu_{p}-c_{p t}(1+\vartheta) \alpha_{w p} \mu_{p}\left(\frac{\lambda_{p}}{n_{p} \mu_{p}}\right)^{\gamma_{p}}$

The expression above gives the maximum value of $c_{p o}$.

$E q(5.5)$ is rearranged and expressed below,

$n_{p} \geq\left(\frac{\lambda_{p}}{\mu_{p}}\right)\left[\frac{c_{p t}(1+\vartheta) \alpha_{w p} \mu_{p}}{c_{p f}+c_{p f f} \mu_{p}-c_{p o}}\right]^{\frac{1}{\gamma_{p}}}$

The expression above gives the minimum value of $n_{p}$.

\section{$\underline{\text { 5.3.3 Objective function for Scenario } 1}$}

All vehicles park on street. the revenue demand equals the arrival rates of parking vehicles, as shown below,

$$
\begin{aligned}
& \lambda_{c r}=\lambda_{c} \\
& \lambda_{p r}=\lambda_{p}
\end{aligned}
$$


Then, the revenue estimation, $E q(5.1)$, becomes,

$y_{r e v}(1)=\frac{c_{c o} \lambda_{c}}{\mu_{c}}+\frac{c_{p o} \lambda_{p}}{\mu_{p}}$

\section{$\underline{\text { 5.3.4 Optimal value of } c_{c a}}$}

Since the revenue is a linear function of $c_{c o}$, the optimal $c_{c o}$ is one of extreme points of a feasible region. $E q(5.3)$ gives the feasible region. The optimal $c_{c o}$ is expressed below,

$c_{c o}^{*}(R 1)=\frac{c_{v} n_{e}}{\tau_{e}}-c_{c t} \alpha_{w c} \mu_{c}\left(\frac{\lambda_{c}}{n_{c} \mu_{c}}\right)^{\gamma_{c}}$

\section{$\underline{\text { 5.3.5 Optimal value of } c_{p o}}$}

Since the revenue is a linear function of $c_{p o}$, the optimal $c_{p o}$ is one of extreme points of a feasible region. $E q(5.6)$ gives the feasible region. The optimal $c_{p o}$ is expressed below,

$c_{p o}^{*}(R 1)=c_{p f}+c_{p f f} \mu_{p}-c_{p t}(1+\vartheta) \alpha_{w p} \mu_{p}\left(\frac{\lambda_{p}}{n_{p} \mu_{p}}\right)^{\gamma_{p}} \quad E q(5.10)$

\subsubsection{Optimal value of $n_{c}$ and $n_{p}$}

Since the revenue is not a function of $n_{c}$ and $n_{p}$, both $n_{c}$ and $n_{p}$ cannot be optimized in Scenario 1. However, the feasible region for $n_{c}, E q(5.4)$, and the feasible region $n_{p}, E q(5.7)$, are still useful for giving ranges of $n_{c}$ and $n_{p}$ for Scenario 1. From the relationship between $n_{c}$ and $n_{p},\left(n_{c} l_{c}+n_{p} l_{p}=l_{b}\right)$, the feasible region for $n_{c}$ becomes,

$n_{p} \leq \frac{l_{b}}{l_{p}}-\frac{l_{c}}{l_{p}}\left(\frac{\lambda_{c}}{\mu_{c}}\right)\left(\frac{c_{c t} \alpha_{w c} \mu_{c}}{\frac{c_{v} n_{e}}{\tau_{e}}-c_{c o}}\right)^{\frac{1}{\gamma_{c}}}$

Therefore, the feasible region for $n_{p}$ becomes, 
$n_{p}(R 1) \in\left\{\left(\frac{\lambda_{p}}{\mu_{p}}\right)\left[\frac{c_{p t}(1+\vartheta) \alpha_{w p} \mu_{p}}{c_{p f}+c_{p f f} \mu_{p}-c_{p o}}\right]^{\frac{1}{\gamma_{p}}}, \frac{l_{b}}{l_{p}}-\frac{l_{c}}{l_{p}}\left(\frac{\lambda_{c}}{\mu_{c}}\right)\left(\frac{c_{c t} \alpha_{w c} \mu_{c}}{\frac{c_{v} n_{e}}{\tau_{e}}-c_{c o}}\right)^{\frac{1}{\gamma_{c}}} E q(5.12)\right.$

\subsection{Revenue model for Scenario 2: Some passenger cars park off street}

Of the model for Scenario 2, the revenue optimization begins with constraints on the decision variables. The next step is to take the objective function into account to find the optimal solutions.

\subsubsection{Constraints on commercial vehicle parking}

These constraints of Scenario 2 are the same as the constraints of Scenario 1 on Section 5.3.1. The constraints on $c_{c o}$ and $n_{c}$ are expressed below,

$$
\begin{aligned}
& c_{c o} \leq \frac{c_{v} n_{e}}{\tau_{e}}-c_{c t} \alpha_{w c} \mu_{c}\left(\frac{\lambda_{c}}{n_{c} \mu_{c}}\right)^{\gamma_{c}} \\
& n_{c} \geq\left(\frac{\lambda_{c}}{\mu_{c}}\right)\left(\frac{c_{c t} \alpha_{w c} \mu_{c}}{\frac{c_{v} n_{e}}{\tau_{e}}-c_{c o}}\right)^{\frac{1}{\gamma_{c}}}
\end{aligned}
$$

\subsubsection{Constraint on passenger car parking}

Some cars park off street. The car revenue demand $\left(\lambda_{p r}\right)$ equals the car breakeven demand $\left(\lambda_{p}^{*}\right)$. The cost of on-street parking equals the cost of off-street parking. From Section 4.5 , equality of the two costs is expressed below,

$\frac{c_{p o}}{\mu_{p}}+c_{p t}(1+\vartheta) \alpha_{w p}\left(\frac{\lambda_{p}^{*}}{n_{p} \mu_{p}}\right)^{\gamma_{p}}=\frac{c_{p f}}{\mu_{p}}+c_{p f f}$

$E q(5.15)$ is rearranged and expressed below,

$$
\lambda_{p}^{*}(N J)=n_{p} \mu_{p}\left[\frac{c_{p f}+c_{p f f} \mu_{p}-c_{p o}}{c_{p t}(1+\vartheta) \alpha_{w p} \mu_{p}}\right]^{\frac{1}{\gamma_{p}}}
$$




\subsubsection{Objective function for Scenario 2}

All commercial vehicles park on street. The commercial revenue demand $\left(\lambda_{c r}\right)$ equals the arrival rates of commercial parking vehicles $\left(\lambda_{c}\right)$. However, some passenger cars park on street. The car revenue demand $\left(\lambda_{p r}\right)$ equals the car breakeven demand $\left(\lambda_{p}^{*}\right)$, as shown below,

$\lambda_{c r}=\lambda_{c}$

$\lambda_{p r}=\lambda_{p}^{*}$

Then, the revenue function becomes,

$y_{\text {rev }}(2)=\frac{c_{c o} \lambda_{c}}{\mu_{c}}+\frac{c_{p o} \lambda_{p}^{*}}{\mu_{p}}$

The car breakeven demand $\left(\lambda_{p}^{*}\right)$ is estimated with $E q(5.16)$. The revenue function becomes,

$y_{\text {rev }}(2)=\frac{c_{c o} \lambda_{c}}{\mu_{c}}+n_{p} c_{p o}\left[\frac{c_{p f}+c_{p f f} \mu_{p}-c_{p o}}{c_{p t}(1+\vartheta) \alpha_{w p} \mu_{p}}\right]^{\frac{1}{\gamma_{p}}} \quad E q(5.17)$

\subsubsection{Optimal value of $c_{c o}$}

Since the revenue is a linear function of $c_{c o}$, the optimal $c_{c o}$ is one of extreme points of a feasible region. $E q(5.13)$ gives the feasible region. The optimal $c_{c o}$ is expressed below,

$c_{c o}^{*}(R 2)=\frac{c_{v} n_{e}}{\tau_{e}}-c_{c t} \alpha_{w c} \mu_{c}\left(\frac{\lambda_{c}}{n_{c} \mu_{c}}\right)^{\gamma_{c}}$ 


\subsubsection{Optimal value of $c_{p o}$}

The revenue is an unconstrained non-linear function of $c_{p o} . c_{p o}$ is optimized based on the necessary condition for a critical point. The partial derivative of the revenue function with respect to $c_{p o}$ is zero.

$$
\frac{\partial y_{r e v}}{\partial c_{p o}}=\frac{n_{p}\left[\gamma_{p} c_{p f}+\gamma_{p} c_{p f f} \mu_{p}-\left(\gamma_{p}+1\right) c_{p o}\right]}{\gamma_{p}\left[c_{p t}(1+\vartheta) \alpha_{w p} \mu_{p}\right]^{\frac{1}{\gamma_{p}}}\left[c_{p f}+c_{p f f} \mu_{p}-c_{p o}\right]^{\frac{\gamma_{p}-1}{\gamma_{p}}}}=0
$$

From the equation above, the optimal $c_{p o}$ is expressed below,

$$
c_{p o}^{*}(R 2)=\frac{\gamma_{p}\left(c_{p f}+c_{p f f} \mu_{p}\right)}{\left(\gamma_{p}+1\right)}
$$

The optimality condition is checked with the sufficient condition,

$$
\frac{\partial^{2} y_{r e v}}{\partial c_{p o}^{2}}=-\frac{\left(\mathrm{l}_{\mathrm{b}}-n_{c} \mathrm{l}_{\mathrm{c}}\right)\left(\mathrm{c}_{\mathrm{pf}}+\mathrm{c}_{\mathrm{pff}} \mu_{\mathrm{p}}\right)}{\gamma_{p} \mathrm{l}_{\mathrm{p}}\left[\mathrm{c}_{\mathrm{pt}}(1+\vartheta) \alpha_{\mathrm{wp}} \mu_{\mathrm{p}}\right]^{\frac{1}{\gamma_{p}}}}\left(\frac{\gamma_{p}+1}{c_{\mathrm{pf}}+\mathrm{c}_{\mathrm{pff}} \mu_{\mathrm{p}}}\right)^{\frac{2 \gamma_{p}-1}{\gamma_{p}}}<0
$$

Therefore, the solution is a local maximum.

\subsubsection{Optimal value of $n_{c}$ and $n_{p}$}

Since the revenue is a linear function of $n_{p}$, the optimal $n_{p}$ is one of extreme points of a feasible region. $E q(5.14)$ gives the feasible region of $n_{c}$. From the relationship between $n_{c}$ and $n_{p},\left(n_{c} l_{c}+n_{p} l_{p}=l_{b}\right)$, the feasible region for $n_{c}$ becomes,

$n_{p} \leq \frac{l_{b}}{l_{p}}-\frac{l_{c}}{l_{p}}\left(\frac{\lambda_{c}}{\mu_{c}}\right)\left(\frac{c_{c t} \alpha_{w c} \mu_{c}}{\frac{c_{v} n_{e}}{\tau_{e}}-c_{c o}}\right)^{\frac{1}{\gamma_{c}}}$

The revenue is an increasing function of $n_{p}$. Therefore, the optimal solution is, 
$n_{p}^{*}(R 2)=\frac{l_{b}}{l_{p}}-\frac{l_{c}}{l_{p}}\left(\frac{\lambda_{c}}{\mu_{c}}\right)\left(\frac{c_{c t} \alpha_{w c} \mu_{c}}{\frac{c_{v} n_{e}}{\tau_{e}}-c_{c o}}\right)^{\frac{1}{\gamma_{c}}}$

\subsection{Revenue model for Scenario 3: Some commercial vehicles double park}

Of the model for Scenario 3, the revenue optimization begins with constraints on the decision variables. The next step is to take the objective function into account to find the optimal solutions.

\subsubsection{Constraints on commercial vehicle parking}

Some commercial vehicles double park. The commercial revenue demand $\left(\lambda_{c r}\right)$ equals the commercial breakeven demand $\left(\lambda_{c}^{*}\right)$. The cost of on-street parking equals the cost of double parking. From Section 4.5, equality of the two costs is expressed below,

$\frac{c_{c o}}{\mu_{c}}+c_{c t} \alpha_{w c}\left(\frac{\lambda_{c}^{*}}{n_{c} \mu_{c}}\right)^{\gamma_{c}}=\frac{c_{v} n_{e}}{\mu_{c} \tau_{e}}$

$E q(5.21)$ is rearranged and expressed below,

$\lambda_{c}^{*}=n_{c} \mu_{c}\left(\frac{c_{v} n_{e}-c_{c o} \tau_{e}}{c_{c t} \alpha_{w c} \mu_{c} \tau_{e}}\right)^{\frac{1}{\gamma_{c}}}$

The number of commercial vehicles double-parking (in other words, the number of double-parking events) equals $\left(\lambda_{c}-\lambda_{c r}\right)$. As explained in Section 4.3, double-parking events delay through traffic, including the parking passenger cars. This leads to an increase in travel cost of the parking passenger cars, which is shown in Section 5.5.2.

\subsubsection{Constraints on passenger car parking}

All passenger cars park on street because the cost of on-street parking is not greater than the cost of off-street parking, which is expressed below, 
$\frac{c_{p o}}{\mu_{p}}+c_{p t}(1+\vartheta) \alpha_{w p}\left(\frac{\lambda_{p}}{n_{p} \mu_{p}}\right)^{\gamma_{p}}+c_{p t} f_{d b} t_{s}\left(\lambda_{c}-\lambda_{c}^{*}\right) \leq \frac{c_{p f}}{\mu_{p}}+c_{p f f}$

From the expression above, the cost of on-street parking includes the cost due to an increase in travel time. $E q(5.23)$ is rearranged and expressed below,

$c_{p o} \leq c_{p f}+c_{p f f} \mu_{p}-c_{p t}(1+\vartheta) \alpha_{w p} \mu_{p}\left(\frac{\lambda_{p}}{n_{p} \mu_{p}}\right)^{\gamma_{p}}-c_{p t} \mu_{p} f_{d b} t_{s}\left(\lambda_{c}-\lambda_{c}^{*}\right) \quad E q(5.24)$

The expression above gives the maximum value of $c_{p o}$.

$E q(5.23)$ is rearranged and expressed below,

$n_{p} \geq\left(\frac{\lambda_{p}}{\mu_{p}}\right)\left[\frac{c_{p t}(1+\vartheta) \alpha_{w p} \mu_{p}}{c_{p f}+c_{p f f} \mu_{p}-c_{p o}-c_{p t} \mu_{p} f_{d b} t_{s}\left(\lambda_{c}-\lambda_{c}^{*}\right)}\right]^{\frac{1}{\gamma_{p}}}$

The expression above gives the minimum value of $n_{p}$.

\subsubsection{Objective function for Scenario 3}

Some commercial vehicles park on street. The commercial revenue demand $\left(\lambda_{c r}\right)$ equals the commercial breakeven demand $\left(\lambda_{c}^{*}\right)$. All the passenger cars park on street. The car revenue demand $\left(\lambda_{p r}\right)$ equals the arrival rate of cars $\left(\lambda_{p}\right)$, as shown below,

$\lambda_{c r}=\lambda_{c}^{*}$

$\lambda_{p r}=\lambda_{p}$

Then, the revenue function becomes,

$y_{\text {rev }}=\frac{c_{c o} \lambda_{c}^{*}}{\mu_{c}}+\frac{c_{p o} \lambda_{p}}{\mu_{p}}$

The commercial breakeven demand $\left(\lambda_{c}^{*}\right)$ is estimated with $E q(5.22)$. The revenue function becomes,

$y_{r e v}(3)=c_{c o} n_{c}\left(\frac{c_{v} n_{e}-c_{c o} \tau_{e}}{c_{c t} \alpha_{w c} \mu_{c} \tau_{e}}\right)^{\frac{1}{\gamma_{c}}}+\frac{c_{p o} \lambda_{p}}{\mu_{p}}$ 


\subsubsection{Optimal value of $c_{c o}$}

The revenue is an unconstrained non-linear function of $c_{c o} . c_{c o}$ is optimized based on the necessary condition,

$$
\frac{\partial y_{r e v}}{\partial c_{c o}}=\frac{n_{c}\left[\gamma_{c} c_{v} n_{e}-\left(\gamma_{c}+1\right) c_{c o} \tau_{e}\right]}{\gamma_{c}\left(c_{c t} \alpha_{w c} \mu_{c} \tau_{e}\right)^{\frac{1}{\gamma_{c}}}\left(c_{v} n_{e}-c_{c o} \tau_{e}\right)^{\frac{\gamma_{c}-1}{\gamma_{c}}}}=0
$$

From the equation above, the optimal $c_{c o}$ is expressed below,

$$
c_{c o}^{*}(R 3)=\frac{\gamma_{c} c_{v} n_{e}}{\left(\gamma_{c}+1\right) \tau_{e}}
$$

The optimality condition is checked with the sufficient condition,

$$
\frac{\partial^{2} y_{r e v}}{\partial c_{c o}^{2}}=-\frac{c_{v} n_{c} n_{e} \tau_{e}}{\gamma_{c}\left(c_{c t} \alpha_{w c} \mu_{c} \tau_{e}\right)^{\frac{1}{\gamma_{c}}}}\left(\frac{\gamma_{c}+1}{c_{v} n_{e}}\right)^{\frac{2 \gamma_{c}-1}{\gamma_{c}}}<0
$$

Therefore, the solution is a local maximum.

\subsubsection{Optimal value of $c_{p o}$}

Since the revenue is a linear function of $c_{p o}$, the optimal $c_{p o}$ is one of extreme points of a feasible region. $E q(5.24)$ gives the feasible region. The optimal $c_{p o}$ is expressed below,

$$
c_{p o}^{*}(R 3)=c_{p f}+c_{p f f} \mu_{p}-c_{p t}(1+\vartheta) \alpha_{w p} \mu_{p}\left(\frac{\lambda_{p}}{n_{p} \mu_{p}}\right)^{\gamma_{p}}-c_{p t} \mu_{p} f_{d b} t_{s}\left(\lambda_{c}-\lambda_{c}^{*}\right)
$$

$$
E q(5.28)
$$

\subsubsection{Optimal value of $n_{c}$ and $n_{p}$}

Since the revenue is a linear function of $n_{c}$, the optimal $n_{c}$ is one of extreme points of a feasible region. $E q(5.25)$ gives the feasible region of $n_{p}$. The revenue is an increasing linear function of $n_{c}$. The higher the $n_{c}$, the higher the revenue. From the relationship 
between $n_{c}$ and $n_{p},\left(n_{c} l_{c}+n_{p} l_{p}=l_{b}\right), n_{p}$ is negatively related to $n_{c}$. Therefore, the lower the $n_{p}$, the higher the revenue. The optimal $n_{p}$ becomes,

$n_{p}^{*}(R 3)=\lambda_{p}\left[\frac{c_{p t}(1+\vartheta) \alpha_{w p}}{c_{p f}+c_{p f f} \mu_{p}-c_{p o}-c_{p t} \mu_{p} f_{d b} t_{s}\left(\lambda_{c}-\lambda_{c}^{*}\right)}\right]^{\frac{1}{\gamma_{p}}} \quad E q(5.29)$

\subsection{Revenue model for Scenario 4: All parking options are selected}

Of the model for Scenario 4, the solution optimization begins with constraints on the decision variables. The next step is to take the objective function into account to find the optimal solutions.

\subsubsection{Constraints on commercial vehicle parking}

These constraints of Scenario 4 are the same as the constraints of Scenario 2 on Section 5.5.1. The estimated commercial breakeven demand equals,

$$
\lambda_{c}^{*}=n_{c} \mu_{c}\left(\frac{c_{v} n_{e}-c_{c o} \tau_{e}}{c_{c t} \alpha_{w c} \mu_{c} \tau_{e}}\right)^{\frac{1}{\gamma_{c}}}
$$

\subsubsection{Constraint on passenger car parking}

Some cars park off street. The car revenue demand $\left(\lambda_{p r}\right)$ equals the car breakeven demand $\left(\lambda_{p}^{*}\right)$. The cost of on-street parking equals the cost of off-street parking. From Section 4.5 , equality of the two costs is expressed below,

$$
\frac{c_{p o}}{\mu_{p}}+c_{p t}(1+\vartheta) \alpha_{w p}\left(\frac{\lambda_{p}^{*}}{n_{p} \mu_{p}}\right)^{\gamma_{p}}+c_{p t} f_{d b} t_{s}\left(\lambda_{c}-\lambda_{c}^{*}\right)=\frac{c_{p f}}{\mu_{p}}+c_{p f f}
$$

$E q(5.31)$ is rearranged and expressed below,

$$
\lambda_{p}^{*}(J)=n_{p} \mu_{p}\left[\frac{1}{c_{p t}(1+\vartheta) \alpha_{w p}}\left(\frac{c_{p f}}{\mu_{p}}+c_{p f f}-\frac{c_{p o}}{\mu_{p}}-c_{p t} f_{d b} t_{s}\left(\lambda_{c}-\lambda_{c}^{*}\right)\right)\right]^{\frac{1}{\gamma_{p}}} E q(5.32)
$$




\subsubsection{Objective function for Scenario 4}

Some commercial vehicles park on street. The commercial revenue demand $\left(\lambda_{c r}\right)$ equals the commercial breakeven demand $\left(\lambda_{c}^{*}\right)$. Some passenger cars park on street. The car revenue demand $\left(\lambda_{p r}\right)$ equals the car breakeven demand $\left(\lambda_{p}^{*}\right)$, as shown below,

$$
\begin{aligned}
& \lambda_{c r}=\lambda_{c}^{*} \\
& \lambda_{p r}=\lambda_{p}^{*}
\end{aligned}
$$

Then, the revenue function becomes,

$$
y_{\text {rev }}=\frac{c_{c o} \lambda_{c}^{*}}{\mu_{c}}+\frac{c_{p o} \lambda_{p}^{*}}{\mu_{p}}
$$

The commercial breakeven demand $\left(\lambda_{c}^{*}\right)$ is estimated with $E q(5.30)$ and the car breakeven demand $\left(\lambda_{p}^{*}\right)$ is estimated with $E q(5.32)$. The revenue function becomes,

$$
\begin{aligned}
y_{r e v}(4)=c_{c o} n_{c}\left(\frac{c_{v} n_{e}-c_{c o} \tau_{e}}{c_{c t} \alpha_{w c} \mu_{c} \tau_{e}}\right)^{\frac{1}{\gamma_{c}}} \quad E q(5.33) \\
+c_{p o} n_{p}\left[\frac{1}{c_{p t}(1+\vartheta) \alpha_{w p}}\left(\frac{c_{p f}}{\mu_{p}}+c_{p f f}-\frac{c_{p o}}{\mu_{p}}-c_{p t} f_{d b} t_{s}\left(\lambda_{c}-\lambda_{c}^{*}\right)\right)\right]^{\frac{1}{\gamma_{p}}}
\end{aligned}
$$

Let $A=\left(c_{c t} \alpha_{w c} \mu_{c}\right)^{\frac{1}{\gamma_{c}}}$

Let $B=\left(c_{p t}(1+\vartheta) \alpha_{w p} \mu_{p}\right)^{\frac{1}{\gamma_{p}}}$

$$
\begin{aligned}
& \text { Let } D=c_{p f}+c_{p f f} \mu_{p}-c_{p o}-c_{p t} f_{d b} t_{s} \mu_{p}\left\{\lambda_{c}-n_{c} \mu_{c}\left(\frac{c_{v} n_{e}-c_{c o} \tau_{e}}{c_{c t} \alpha_{w c} \mu_{c} \tau_{e}}\right)^{\frac{1}{\gamma_{c}}}\right\} \\
& y_{\text {rev }}(4)=\frac{c_{c o} n_{c}}{A}\left(\frac{c_{v} n_{e}}{\tau_{e}}-c_{c o}\right)^{\frac{1}{\gamma_{c}}}+\frac{c_{p o} n_{p}}{B} D^{\frac{1}{\gamma_{p}}} \quad E q(5.34)
\end{aligned}
$$




\subsubsection{Optimal value of $c_{c o}$}

The revenue is an unconstrained non-linear function of $c_{c o} . c_{c o}$ is optimized based on the necessary condition,

$$
\begin{gathered}
\frac{\partial y_{r e v}}{\partial c_{c o}}=\frac{n_{c}}{A}\left(\frac{c_{v} n_{e}}{\tau_{e}}-c_{c o}\right)^{\frac{1}{\gamma_{c}}}-\frac{c_{c o} n_{c}}{\gamma_{c} A\left(\frac{c_{v} n_{e}}{\tau_{e}}-c_{c o}\right)^{\frac{\gamma_{c}-1}{\gamma_{c}}}} \\
-\left[\frac{c_{p o} n_{p}}{\gamma_{p} B D^{\frac{\gamma_{p}-1}{\gamma_{p}}}} \cdot \frac{c_{p t} f_{d b} \mu_{p} n_{c}}{\gamma_{c} A\left(\frac{c_{v} n_{e}}{\tau_{e}}-c_{c o}\right)^{\frac{\gamma_{c}-1}{\gamma_{c}}}}\right]=0
\end{gathered}
$$

$\gamma_{c}\left(\frac{c_{v} n_{e}}{\tau_{e}}\right)-\left(\gamma_{c}+1\right) c_{c o}^{*}-c_{p t} f_{d b} \mu_{p}\left(\frac{c_{p o} n_{p}}{\gamma_{p} B}\right) D\left(c_{c o}^{*}\right)^{\frac{1-\gamma_{p}}{\gamma_{p}}}=0 \quad E q(5.35)$

If $\gamma_{c}=\gamma_{p}=1$,

$$
c_{c o}^{*}\left(R 4 \mid \gamma_{c}=\gamma_{p}=1\right)=\frac{c_{v} n_{e}}{2 \tau_{e}}-\frac{f_{d b} n_{p} c_{p o}}{2(1+\vartheta) \alpha_{w p}} \quad E q(5.36)
$$

The optimality condition is checked with the sufficient condition,

$$
\frac{\partial^{2} y_{r e v}}{\partial c_{c o}^{2}}=-\left(\gamma_{c}+1\right)-\frac{\gamma_{p}}{\gamma_{p}-1} c_{p t} f_{d b} \mu_{p}\left(\frac{c_{p o} n_{p}}{\gamma_{p} B}\right)\left(D^{\frac{1-2 \gamma_{p}}{\gamma_{p}}}\right) \cdot \frac{c_{p t} f_{d b} \mu_{p} n_{c}}{\gamma_{c} A\left(\frac{c_{v} n_{e}}{\tau_{e}}-c_{c o}\right)^{\frac{\gamma_{c}-1}{\gamma_{c}}}}<0
$$

Therefore, the solution is a local maximum.

\section{$\underline{\text { 5.6.5 Optimal value of } c_{p o}}$}

The revenue is an unconstrainted non-linear function of $c_{p o} . c_{p o}$ is optimized based on the necessary condition, 


$$
\begin{aligned}
& \frac{\partial y_{\text {rev }}}{\partial c_{p o}}=\frac{n_{p}}{B} D^{\frac{1}{\gamma_{p}}}-\frac{c_{p o} n_{p}}{\gamma_{p} B D^{\frac{\gamma_{p}-1}{\gamma_{p}}}}=0 \\
& \begin{array}{l}
D-\frac{c_{p o}^{*}}{\gamma_{p}}=0 \quad E q(5.37) \\
\text { If } \gamma_{c}=\gamma_{p}=1, \\
c_{p o}^{*}\left(R 4: \gamma_{c}=\gamma_{p}=1\right)=\frac{c_{p f}+c_{p f f} \mu_{p}}{2}-\frac{c_{p t} f_{d b} t_{s} \mu_{p}}{2}\left\{\lambda_{c}-\frac{n_{c}}{c_{c t} \alpha_{w c}}\left(\frac{c_{v} n_{e}}{\tau_{e}}-c_{c o}\right)\right\} \\
E q(5.38)
\end{array}
\end{aligned}
$$

The optimality condition is checked with the sufficient condition,

$$
\frac{\partial^{2} y_{r e v}}{\partial c_{p o}^{2}}=-\frac{n_{p}}{\gamma_{p} B D^{\frac{\gamma_{p}-1}{\gamma_{p}}}}-\frac{n_{p}}{\gamma_{p} B D^{\frac{\gamma_{p}-1}{\gamma_{p}}}}-\frac{c_{p o} n_{p}}{\left(\gamma_{p}-1\right) B D^{\frac{-2 \gamma_{p}+1}{\gamma_{p}}}}<0
$$

The solution is a local maximum.

\subsubsection{Optimal value of $n_{c}$ and $n_{p}$}

To simplify the revenue function, let $\gamma_{c}$ and $\gamma_{p}$ equal to 1.0. From Section 4.4, the number of loading zones $\left(n_{c}\right)$ is a function of the number of parking spaces $\left(n_{p}\right)$. Then, the revenue becomes

$$
\begin{aligned}
y_{\text {rev }}(4) & =c_{c o}\left(\frac{l_{b}-n_{p} l_{p}}{l_{c}}\right)\left(\frac{c_{v} n_{e}-c_{c o} \tau_{e}}{c_{c t} \alpha_{w c} \mu_{c} \tau_{e}}\right) \quad E q(5.39) \\
& +\frac{n_{p} c_{p o}}{(1+\vartheta) \alpha_{w p}}\left\{\frac{\left(c_{p f}+c_{p f f} \mu_{p}-c_{p o}\right)}{c_{p t} \mu_{p}}-f_{d b} t_{s}\left[\lambda_{c}-\frac{n_{c}}{c_{c t} \alpha_{w c}}\left(\frac{c_{v} n_{e}}{\tau_{e}}-c_{c o}\right)\right]\right\}
\end{aligned}
$$

The revenue is an unconstrained non-linear function of $n_{p} . n_{p}$ is optimized based on the necessary condition, 


$$
\begin{aligned}
\frac{\partial y_{r e v}}{\partial n_{p}}=0=- & c_{c o}\left(\frac{l_{p}}{l_{c}}\right)\left(\frac{c_{v} n_{e}-c_{c o} \tau_{e}}{c_{c t} \alpha_{w c} \mu_{c} \tau_{e}}\right) \\
& +\frac{c_{p o}}{(1+\vartheta) \alpha_{w p}}\left\{\left(\frac{c_{p f}+c_{p f f} \mu_{p}-c_{p o}}{c_{p t} \mu_{p}}\right)-f_{d b} t_{s} \lambda_{c}\right. \\
& \left.+\frac{f_{d b} t_{s}}{c_{c t} \alpha_{w c}}\left(\frac{l_{b}-2 n_{p} l_{p}}{l_{c}}\right)\left(\frac{c_{v} n_{e}}{\tau_{e}}-c_{c o}\right)\right\}
\end{aligned}
$$

From the equation above, the optimal $n_{p}$ is expressed below,

$$
\begin{aligned}
& n_{p}^{*}\left(R 4: \gamma_{c}=\gamma_{p}=1\right)=\frac{l_{b}}{2 l_{p}}-\lambda_{c} \alpha_{w c}\left(\frac{l_{c}}{2 l_{p}}\right)\left(\frac{c_{c t} \tau_{e}}{c_{v} n_{e}-c_{c o} \tau_{e}}\right) \quad E q(5.40) \\
& -\frac{1}{2}\left(\frac{c_{c o}}{c_{p o}}\right)\left(\frac{(1+\vartheta) \alpha_{w p}}{f_{d b} t_{s} \mu_{c}}\right)+\left(\frac{l_{c}}{2 l_{p}}\right)\left(\frac{\alpha_{w c}}{f_{d b} t_{s} \mu_{p}}\right)\left(\frac{c_{p f}+c_{p f f} \mu_{p}-c_{p o}}{c_{p t}}\right)\left(\frac{c_{c t} \tau_{e}}{c_{v} n_{e}-c_{c o} \tau_{e}}\right)
\end{aligned}
$$

The optimality condition is checked with the sufficient condition,

$$
\frac{\partial^{2} y_{r e v}}{\partial n_{p}^{2}}=-\frac{c_{p o}}{(1+\vartheta) \alpha_{w p}} \frac{f_{d b} t_{s}}{c_{c t} \alpha_{w c}}\left(\frac{2 l_{p}}{l_{c}}\right)\left(\frac{c_{v} n_{e}}{\tau_{e}}-c_{c o}\right)<0
$$

The solution is a local maximum.

\subsection{Solution summary and interpretation}

This section analyzes how the decision variable is affected by parameters and other variables. Before interpreting the solutions, all solutions are summarized in Table 5.1. 
Table 5.1 Solutions to revenue models for $\mathbf{4}$ scenarios

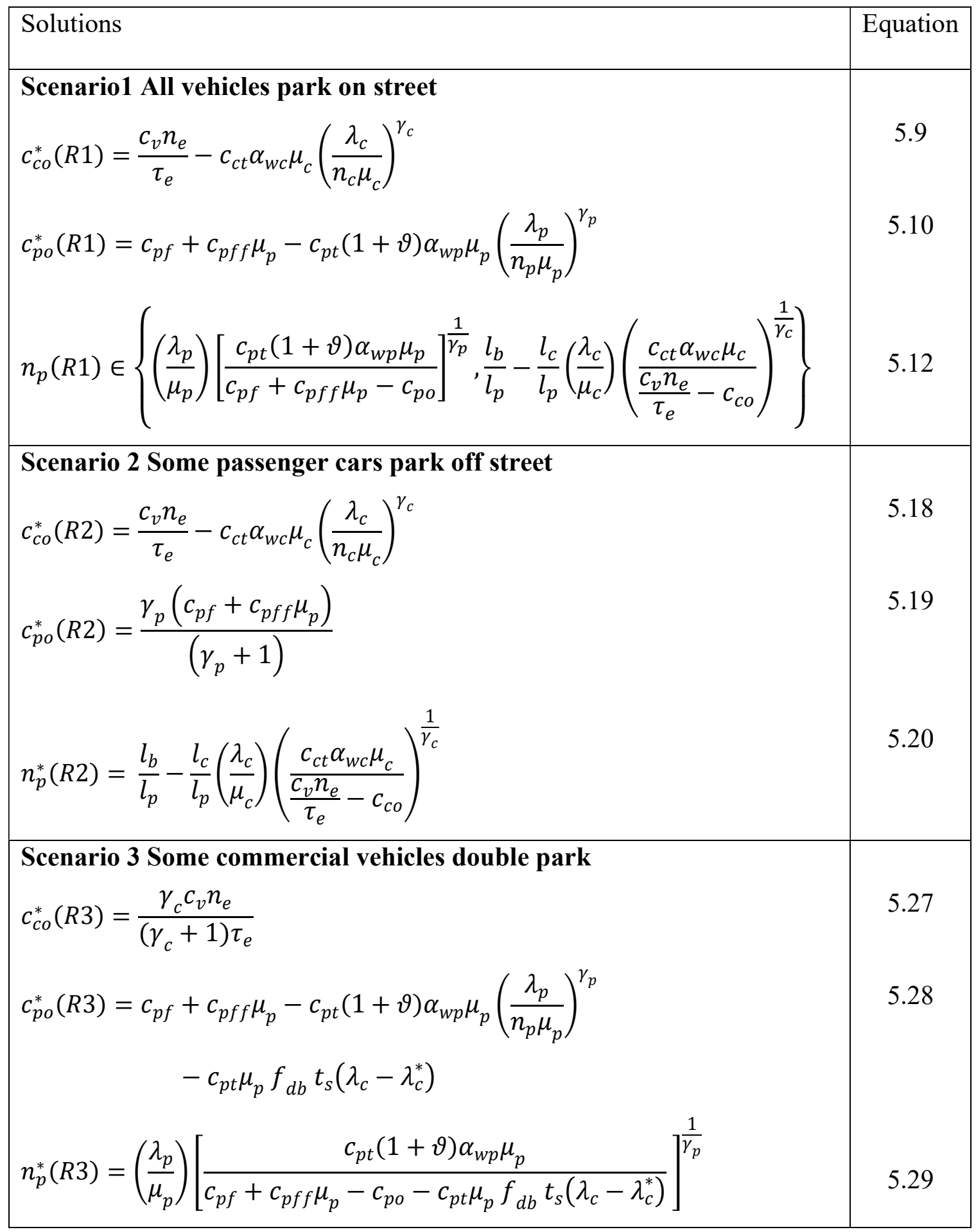


Table 5.1 Solutions to revenue models for $\mathbf{4}$ scenarios (continued)

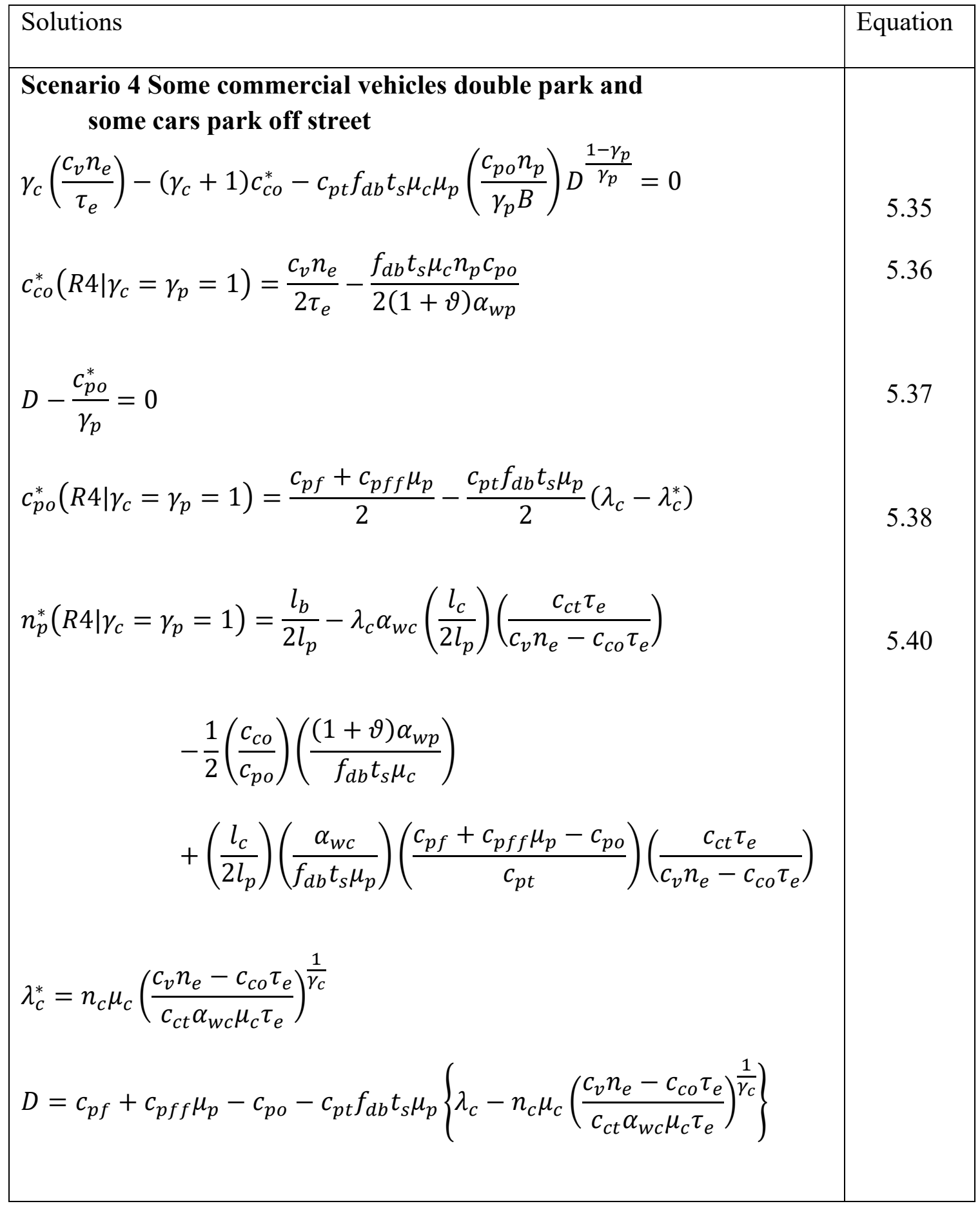


From Table 5.1, The interpretation to variable parameters and decision variables is summarized in Tables 5.2 to 5.4 .

Table 5.2 Interpretation of optimal loading zone meter rate for revenue models

\begin{tabular}{|c|c|}
\hline Variable & Interpretation of optimal $c_{c o}$ \\
\hline$\frac{c_{v} n_{e}}{\tau_{e}} \propto c_{c o}^{*}$ & If the cost of the other parking option is high, the meter rate can be high. \\
\hline \multirow[b]{2}{*}{$n_{c} \propto c_{c o}^{*}$} & $\underline{\text { Scenarios } 1 \text { and } 2}$ \\
\hline & $\begin{array}{l}\text { If parking supply is high, the parking search cost will be low. The other } \\
\text { component of on-street parking, the meter rate, can be high. }\end{array}$ \\
\hline \multirow{2}{*}{$\lambda_{c} \propto \frac{1}{c_{c o}^{*}}$} & $\underline{\text { Scenarios } 1 \text { and } 2}$ \\
\hline & $\begin{array}{l}\text { If parking demand is low, the meter rate can be high and the cost of } \\
\text { on-street parking is still cheaper than the cost of double-parking. }\end{array}$ \\
\hline \multirow{2}{*}{$c_{c t} \propto \frac{1}{c_{c o}^{*}}$} & $\underline{\text { Scenarios } 1 \text { and } 2}$ \\
\hline & $\begin{array}{l}\text { If value of time is high, the parking search cost will be high. The other } \\
\text { component of on-street parking, the meter rate, has to be low. }\end{array}$ \\
\hline
\end{tabular}

Table 5.3 Interpretation of optimal parking space meter rate for revenue models

\begin{tabular}{|c|l|}
\hline Variable & \multicolumn{1}{|c|}{ Interpretation of optimal $c_{p o}$} \\
\hline$c_{p f} \propto c_{p o}^{*}$ & If the cost of the other parking option is high, the meter rate can be high. \\
\hline$n_{p} \propto c_{p o}^{*}$ & $\begin{array}{l}\frac{\text { Scenarios 1 and 3 }}{\text { If parking supply is high, the parking search cost will be low. The other }} \\
\text { component of on-street parking, the meter rate, can be high. }\end{array}$ \\
\hline$\lambda_{p} \propto \frac{1}{c_{p o}^{*}}$ & $\begin{array}{l}\frac{\text { Scenarios 1 and 3 }}{\text { If parking demand is low, the meter rate can be high and the cost of }} \\
\text { on-street parking is still cheaper than the cost of double-parking. }\end{array}$ \\
\hline$c_{p t} \propto \frac{1}{c_{p o}^{*}}$ & $\begin{array}{l}\frac{\text { Scenarios 1 and 3 }}{\text { If value of time is high, the parking search cost will be high. The other }} \\
\text { component of on-street parking, the meter rate, has to be low. }\end{array}$ \\
\hline
\end{tabular}


Table 5.4 Interpretation of optimal curb length allocation for revenue models

\begin{tabular}{|c|c|}
\hline Parameter & Interpretation of optimal $n_{p}$ \\
\hline$l_{b} \propto n_{p}^{*}$ & The longer the block, the more the length is allocated to parking spaces. \\
\hline$\lambda_{c} \propto \frac{1}{n_{p}^{*}}$ & $\begin{array}{l}\text { Scenarios } 2 \text { and } 4 \\
\text { If the arrival rate of commercial vehicles is high, the number of loading } \\
\text { zones will be high. This leads to low number of parking spaces. }\end{array}$ \\
\hline$\lambda_{p} \propto n_{p}^{*}$ & $\begin{array}{l}\text { Scenarios } 1 \text { and } 3 \\
\text { The higher the arrival rate of parking cars, the more the number of parking } \\
\text { spaces to prevent an increase in the parking search cost. }\end{array}$ \\
\hline
\end{tabular}

\subsection{Steps to find optimal solutions}

From the optimal solutions (Section 5.7), for most scenarios, one decision variable is a function of the other two decision variables. It is practical to vary one of the variables and calculate the other variables. $c_{c o}^{*}$ and $c_{p o}^{*}$ are similar because both are meter rates while $n_{c}^{*}$ is different from the others since it is related to supply allocation. Therefore, it is to vary $n_{c}$ and calculate the others. The steps to find optimal solutions are described below,

1) Start with $n_{c}$ equal to 1

2) Calculate $c_{c o}^{*}$ and $c_{p o}^{*}$ using equations shown below,

\begin{tabular}{|c|c|c|}
\hline \multirow{2}{*}{ Scenario } & \multicolumn{2}{|c|}{ Equation for calculating the optimal meter rates } \\
\cline { 2 - 3 } & $c_{c o}^{*}$ & $c_{p o}^{*}$ \\
\hline 1 & Eq 5.9 & Eq 5.10 \\
\hline 2 & Eq 5.18 & Eq 5.19 \\
\hline 3 & Eq 5.27 & Eq 5.28 \\
\hline 4 & Eq 5.35 & Eq 5.37 \\
\hline
\end{tabular}

3) Calculate the revenue

4) Check if $n_{c}$ equals $l_{b} / l_{c}$. If not, increase $n_{c}$ and repeat steps 2,3 , and 4

5) Find $n_{c}$ whose revenue is maximum 


\subsection{Conclusion}

This chapter proposes a revenue maximization model for on-street parking system. The decision variables are meter rate of loading zones $\left(c_{c o}\right)$, meter rate of parking spaces $\left(c_{p o}\right)$, the number of loading zones $\left(n_{c}\right)$, and the number of parking spaces $\left(n_{p}\right)$. The first two variables are to set fees for parking on street. The last two variables are to allocate curb length to loading zones and parking spaces.

One decision variable is optimized at a time while the other variables keep constant. Optimal solutions are different between the scenarios. However, the impacts of parameters on a decision variable in different scenarios are similar: the same direction but different magnitude. In the next chapter, a case study is conducted to demonstrate the optimal solutions and the impacts of parameters for all the parking scenarios. 


\section{Chapter 6 Case Study for Revenue Models}

\subsection{Introduction}

This chapter demonstrates how the revenue models optimize meter rates and curb length allocation under given circumstances. Since there are 4 scenarios for developing parking models (Section 4.6), 4 sets of data are assumed for case studies; one set for one scenario. To facilitate comparisons, most variables are kept the same, including value of time, unit costs, length and service rate of loading zones and parking spaces, and coefficient for estimating parking search time, as shown in Table 6.1. However, demands must be changed.

The difference among the sets of data is arrival rates of parking commercial vehicles and parking passenger cars. Of the first set, the arrival rates of both vehicle types are low. All the vehicles park on street. Of the second set, the arrival rate of commercial vehicles is low but the arrival rate of passenger cars is high. All the commercial vehicles park on street. Some passenger cars park on street and others park off street. Of the third set, the arrival rate of commercial vehicles is high but the arrival rate of passenger cars is low. Some commercial vehicles park on street but others double park. Of the fourth set, the arrival rates of commercial vehicles and passenger cars are high. All parking options (onstreet parking, double-parking, and off-street parking) are chosen. The base data for the four cases is shown in Table 6.2. 
Table 6.1 Base data for case studies

\begin{tabular}{|c|c|c|c|c|}
\hline Notation & Definition & Unit & Value & Source \\
\hline \multicolumn{5}{|c|}{ Commercial vehicle parking } \\
\hline$c_{c t}$ & Commercial vehicle value of time & $\$ /$ hour & 30 & 1 \\
\hline$c_{d b}$ & Traffic delay cost per double-parking event & \$/vehicle & 7.16 & 2 \\
\hline$c_{d c}$ & $\begin{array}{l}\text { Traffic delay cost due to a commercial vehicle } \\
\text { entering and exiting a loading zone }\end{array}$ & \$/vehicle & 0.65 & 2 \\
\hline$c_{v}$ & Parking violation cost (fine) & \$/ticket & 60 & 3 \\
\hline$f_{d b}$ & Double-parking delays to a parking car & hour/event & 0.007 & 2 \\
\hline$l_{b}$ & Available parking length in one block & Feet & 240 & 4 \\
\hline$l_{c}$ & Unit length of loading zones & Feet & 30 & 5 \\
\hline$n_{e}$ & Number of parking enforcement officers & - & 1 & assume \\
\hline$\tau_{e}$ & Parking enforcement cycle period of an officer & Hour/cycle & 4 & assume \\
\hline$\alpha_{w c}$ & Waiting time coefficient for commercial vehicle & & 0.25 & assume \\
\hline$\gamma_{c}$ & Waiting time coefficient for commercial vehicle & & 1,2 & assume \\
\hline$\mu_{c}$ & Service rate of loading zones & Vehicle/hour & 4 & 6 \\
\hline \multicolumn{5}{|c|}{ Passenger car parking } \\
\hline$c_{d p}$ & $\begin{array}{l}\text { Traffic delay cost due to a passenger vehicle } \\
\text { entering and exiting a parking space }\end{array}$ & \$/vehicle & 0.13 & 2 \\
\hline$c_{p f}$ & Meter rate of off-street parking lots & $\$$ /hour & 4 & 7 \\
\hline$c_{p f f}$ & Fixed parking search cost of off-street parking & $\$$ & 0.5 & assume \\
\hline$c_{p t}$ & Passenger car value of time & $\$ /$ hour & 13 & 8 \\
\hline$l_{p}$ & Unit length of parking spaces & feet & 20 & 9 \\
\hline$t_{s}$ & Average on-street parking search time & hour & 0.125 & assume \\
\hline$\alpha_{w p}$ & Waiting time coefficient for passenger cars & & 0.25 & assume \\
\hline$\gamma_{p}$ & Waiting time coefficient for passenger cars & & 1,2 & assume \\
\hline$\vartheta$ & Ratio of walking time to cruising time & - & 0.5 & 10 \\
\hline$\mu_{p}$ & Service rate of parking spaces & Vehicle/hour & 2 & 11 \\
\hline \multicolumn{5}{|c|}{ 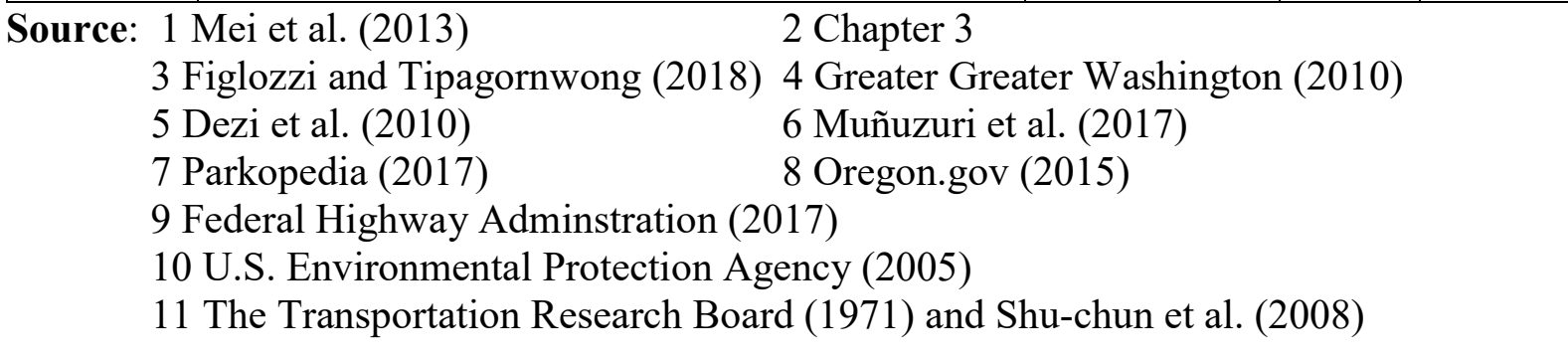 } \\
\hline
\end{tabular}


Table 6.2 Arrival rates of parking vehicle for 4 scenarios

\begin{tabular}{|c|l|c|c|c|}
\hline \multirow{2}{*}{ Scenario } & \multicolumn{1}{|c|}{ Description } & \multicolumn{3}{|c|}{ Arrival rate (vehicles per hour) } \\
\cline { 3 - 5 } & & $\begin{array}{c}\text { Commercial } \\
\text { vehicles }\left(\lambda_{c}\right)\end{array}$ & $\begin{array}{c}\text { Passenger } \\
\text { cars }\left(\lambda_{p}\right)\end{array}$ & $\begin{array}{c}\text { Aggregated } \\
\text { rate }\left(\lambda_{a}\right)\end{array}$ \\
\hline 1 & On street only & 3 & 10 & 12.25 \\
\hline 2 & Some cars park off street & 3 & 20 & 17.50 \\
\hline 3 & Some trucks double park & 10 & 10 & 22.25 \\
\hline 4 & $\begin{array}{l}\text { Some trucks double park and } \\
\text { some cars park off street }\end{array}$ & 10 & 20 & 27.50 \\
\hline
\end{tabular}

Note: the aggregated rate $\lambda_{a}$ is calculated based on equations in Section 4.5.6.

\subsection{Optimal solutions for passenger car parking equivalent}

This section shows how the optimal meter rate of parking spaces changes, responding to change in the aggregated arrival rate of equivalent passenger vehicles $\left(\lambda_{a}\right)$. Since the arrival rate of commercial vehicles is converted to the arrival rate of equivalent passenger vehicles, there are no impacts of vehicle types. In addition, the equivalent curb length allocation is constant. The impact of arrival rates on the meter rate can be analyzed clearly.

From Chapter 4, the parking options for passenger cars are parking on street and parking off street. The two cases are analyzed: parking on street only and parking on- and off-street. For the case of vehicles parking on street only, the scenario-1 revenue model is used to find optimal solutions with the assumption that the arrival rate of commercial vehicles is zero. For the case of vehicles parking on street and off street, the scenario-2 revenue model is used to find optimal solutions with the assumption that the arrival rate of commercial vehicles is zero. The equivalent breakeven demand is calculated based on Section 4.5.6. 


\subsubsection{Parking on street only}

As $\lambda_{a}$ increases, the parking search cost increases. In order to keep the on-street option cheaper than the off-street option, the meter rate has to decrease. The results are shown in Table 6.3. If $\lambda_{a}$ is very high, the meter rate will be negative. This means that drivers get paid in order to park on street. This is not common in many urban areas.

Table 6.3 Solutions of revenue models: vehicle equivalent parking on street

\begin{tabular}{|r|r|r|r|r|}
\hline \multicolumn{1}{|c|}{$\lambda_{a}$} & \multicolumn{1}{|c|}{$c_{p o}^{*}$} & \multicolumn{1}{c|}{$\lambda_{a r}$} & \multicolumn{1}{|c|}{ Revenue } & Social optimum \\
\hline 7.50 & 4.05 & 7.50 & 15.18 & 10.63 \\
\hline 9.92 & 3.33 & 9.92 & 16.54 & 6.99 \\
\hline 12.25 & 2.46 & 12.25 & 15.07 & -2.08 \\
\hline 17.50 & -0.18 & 17.50 & -1.61 & -49.24 \\
\hline 22.25 & -3.38 & 22.25 & -37.60 & -133.72 \\
\hline
\end{tabular}

\section{$\underline{6.2 .2 \text { Parking on street and off street }}$}

As $\lambda_{a}$ increases, the meter rate and the revenue demand do not change. The only change is the number of vehicles parking off street, as shown in Table 6.4.

Table 6.4 Solutions of revenue models: vehicle equivalent parking on- and off-street

\begin{tabular}{|r|r|r|r|r|}
\hline \multicolumn{1}{|c|}{$\lambda_{a}$} & \multicolumn{1}{|c|}{$c_{p o}^{*}$} & \multicolumn{1}{c|}{$\lambda_{a r}$} & Revenue & Social optimum \\
\hline 9.92 & 3.33 & 9.92 & 16.54 & 6.99 \\
\hline 12.25 & 3.33 & 9.92 & 16.54 & 5.04 \\
\hline 17.50 & 3.33 & 9.92 & 16.54 & 0.66 \\
\hline 22.25 & 3.33 & 9.92 & 16.54 & -3.29 \\
\hline
\end{tabular}

Considering Table 6.3 and Table 6.4, it is practical to decrease the meter rate as the arrival rate increases while all the vehicles park on street, according to Table 6.3. When the meter rate equals the meter rate in Table $6-4$, it is practical to keep the meter rate the same and some vehicles park off street. It is not reasonable to keep all vehicles park on 
street by lowering the meter rate because the meter rate can be negative. It can be inferred that drivers are paid to park on street, according to Table 6.3.

\subsection{Optimal solutions for parking scenarios}

Each scenario is optimized at a time. How to maximize the revenue is already explained in Section 5.8. The optimal curb length allocation and its optimal meter rates of both parking types lead to the highest revenue. The numerical results include the optimal meter rates of both parking types, the revenue demand of both vehicle types, and the objective function (the revenue). If the revenue demand or the meter rate slightly change, it will not be shown in plots.

\subsubsection{Optimal solution for scenario-1 revenue model}

The management strategy of scenario 1 is to keep all vehicles park on street. From $E q(5.9)$ and $E q(5.10)$, if the parking utilization is high or the curb allocation is low, the optimal meter rates of loading zones and parking spaces will be low.

Based on data from Table 6.1 and 6.2, for low curb allocation, the optimal meter rate of parking spaces is lower than the optimal meter rate of loading zones, as shown in Figure 6.1. To prevent a low value of revenue, the majority of curb length is allocated to parking spaces, as shown in Figure 6.2. 


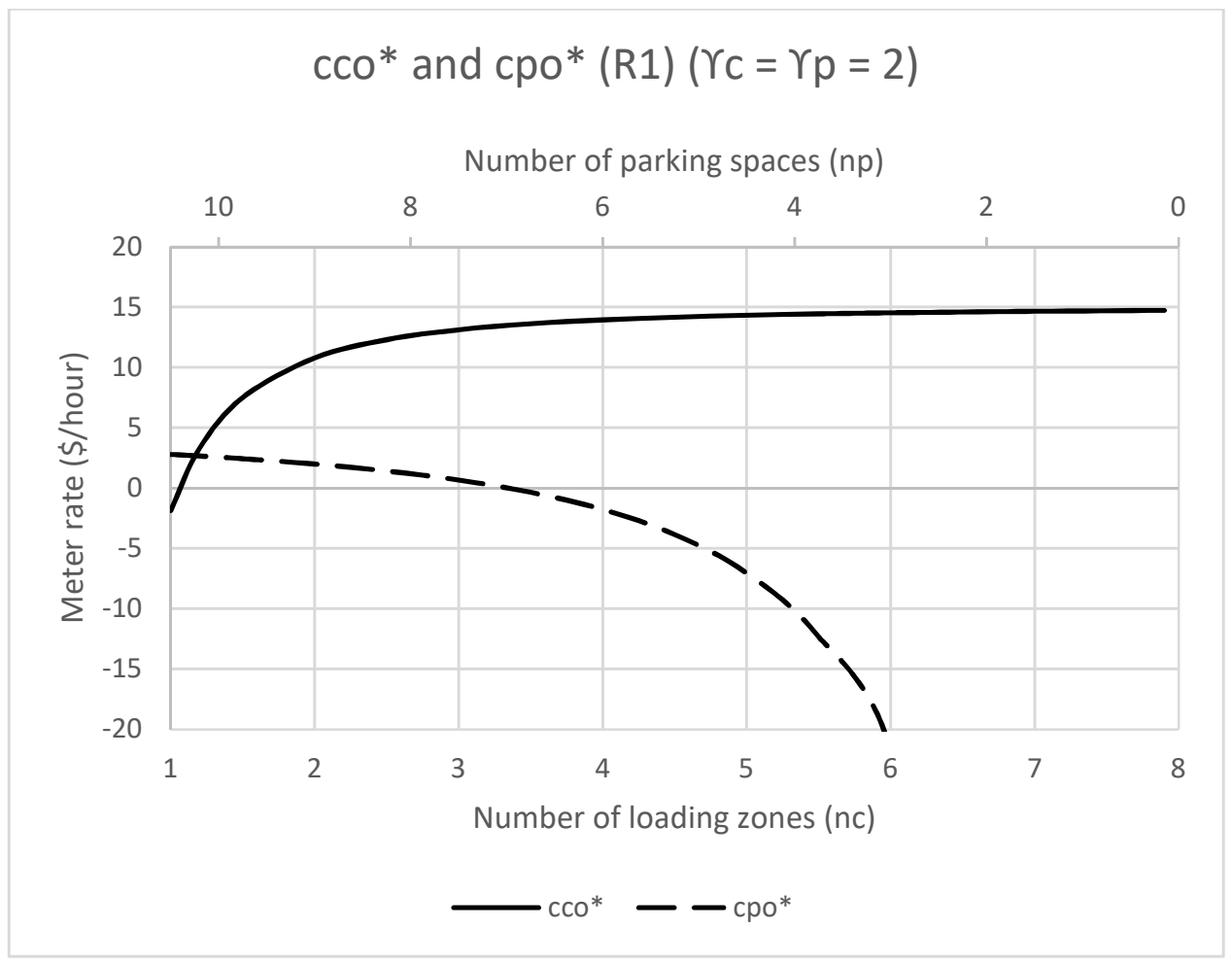

Figure 6.1 Optimal meter rates of scenario-1 revenue model

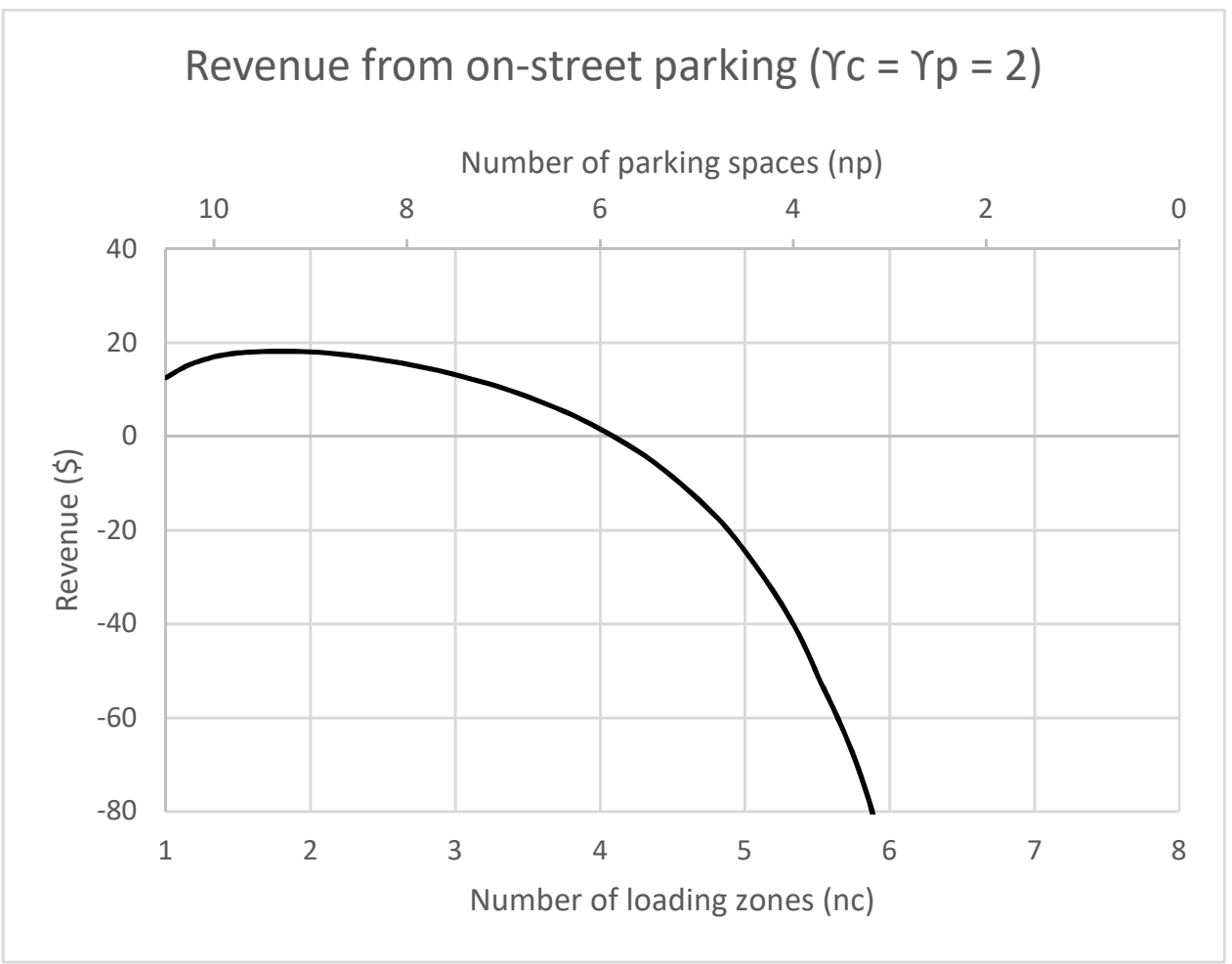

Figure 6.2 On-street scenario-1 parking revenue 


\subsubsection{Optimal solution for scenario-2 revenue model}

The management strategy of scenario- 2 is to keep all commercial vehicles park on street and to let some passenger cars park off street. For loading zones, from $E q(5.18)$, if its curb allocation $\left(n_{c}\right)$ increases, its optimal meter rate of loading zones $\left(c_{c o}^{*}\right)$ will increase. The revenue from loading zones will increase.

For parking spaces, from $E q(5.19)$, the optimal meter rate of parking spaces $\left(c_{p o}^{*}\right)$ is constant. If its curb allocation $\left(n_{p}\right)$ decreases, the car revenue demand $\left(\lambda_{p r}\right)$ will decrease. The revenue from parking spaces will decrease.

Based on data from Table 6.1 and 6.2, if the number of loading zones $\left(n_{c}\right)$ increases, $c_{c o}^{*}$ will increase but $c_{p o}^{*}$ will be constant, as shown in Figure 6.3. $\lambda_{c r}$ will be constant but $\lambda_{p r}$ will decrease, as shown in Figure 6.4. Since the increase in the revenue from loading zones is lower than the decrease in the revenue from parking spaces, majority of curb length is allocated to parking spaces, as shown in Figure 6.5. 


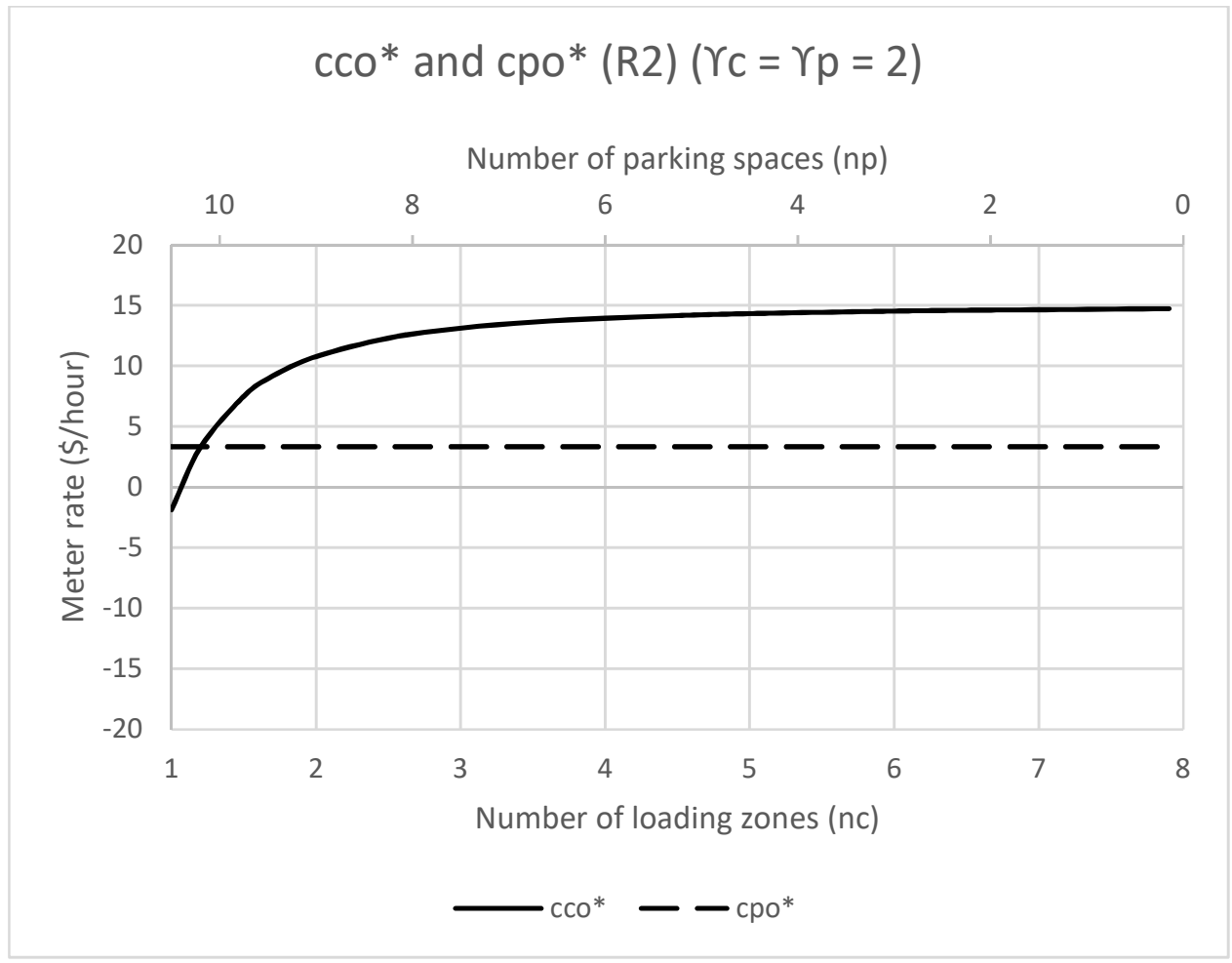

Figure 6.3 Optimal meter rates of scenario-2 revenue model

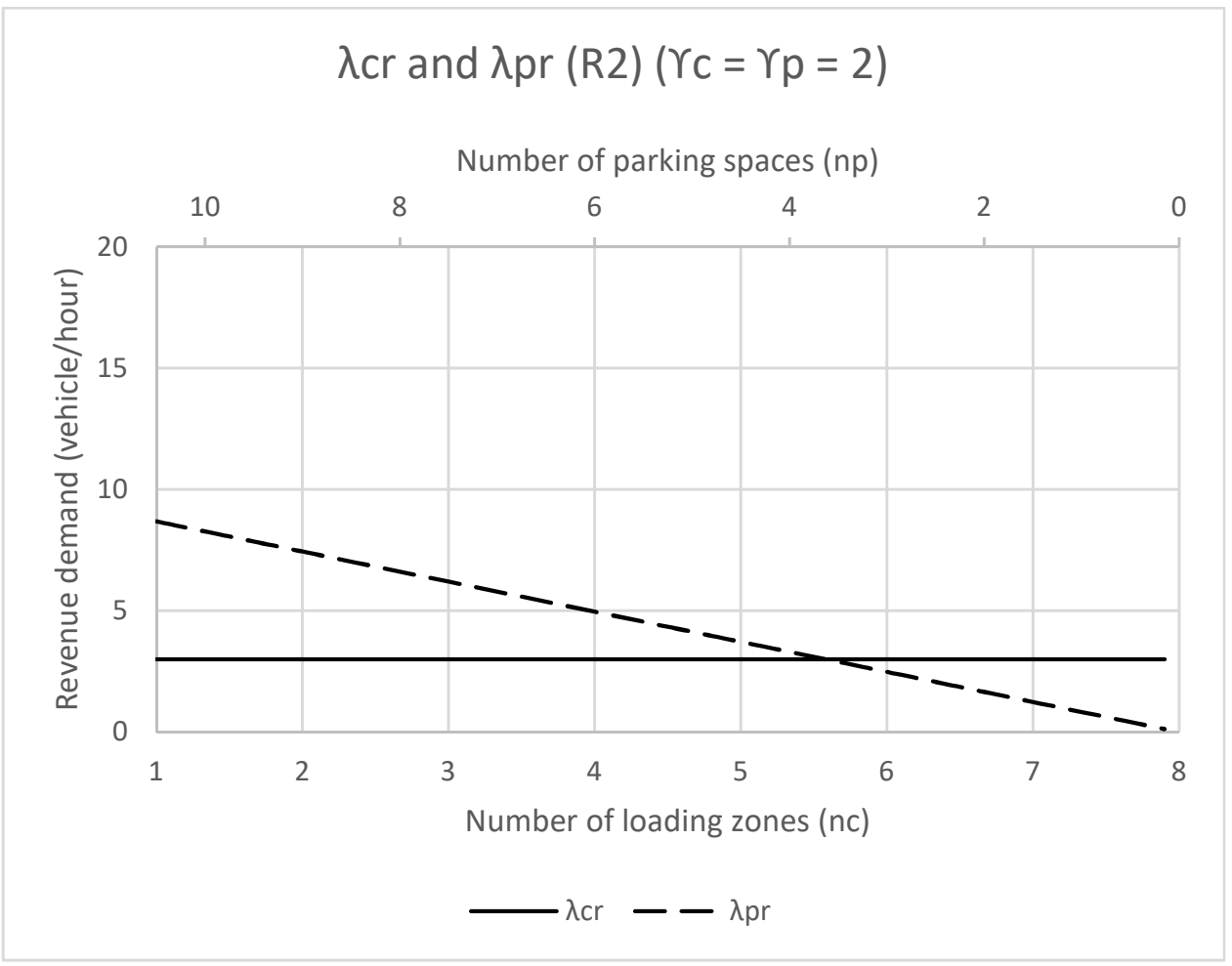

Figure 6.4 Revenue demand of scenario-2 revenue model 


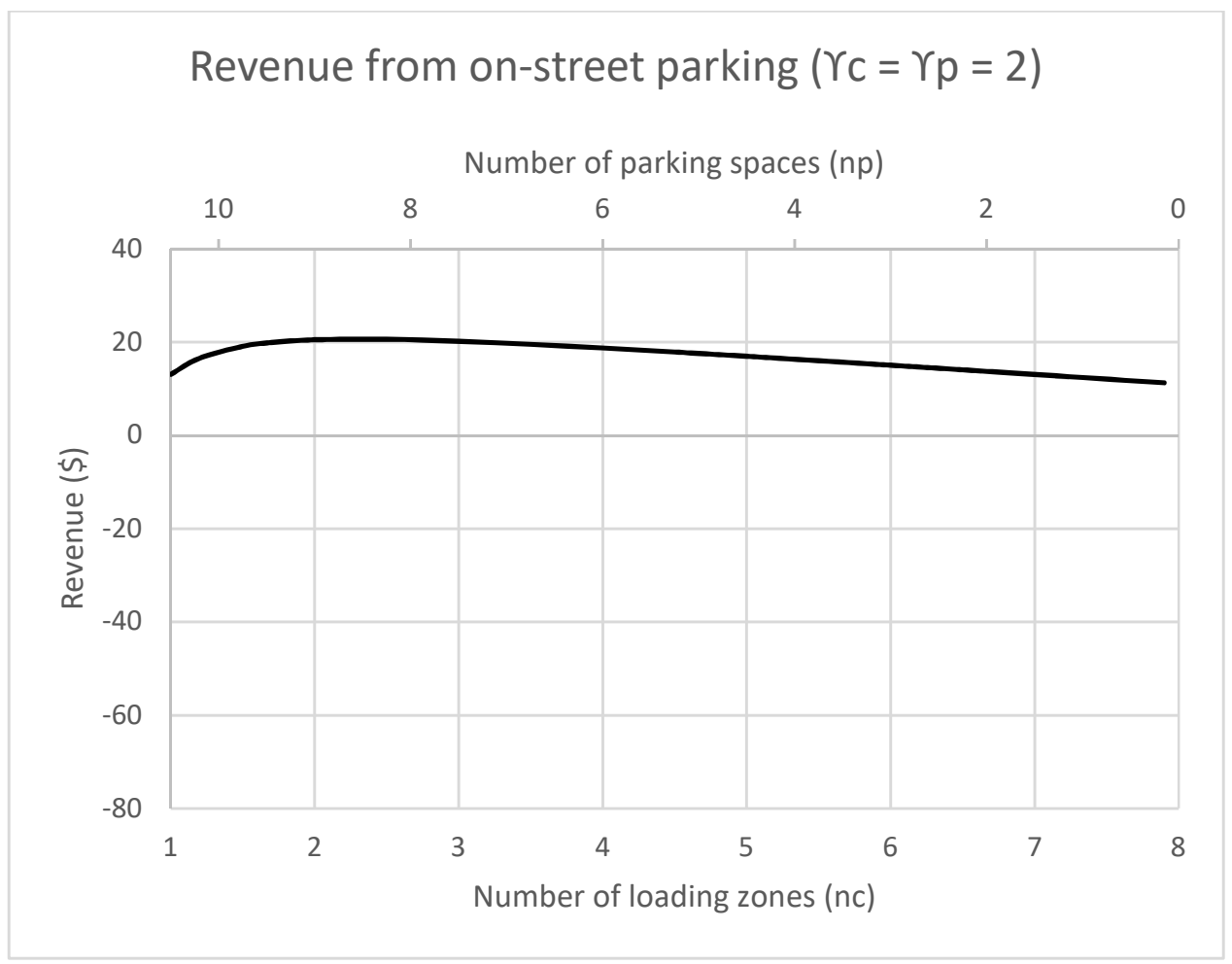

Figure 6.5 On-street scenario-2 parking revenue

\subsubsection{Optimal solution for scenario-3 revenue model}

The management strategy of scenario 3 is to let some commercial vehicles double park and to keep all passenger cars park on street. For loading zones, from $E q(5.27)$, the optimal meter rate of loading zones $\left(c_{c o}^{*}\right)$ is constant. If its curb allocation $\left(n_{c}\right)$ increases, the commercial revenue demand $\left(\lambda_{c r}\right)$ will increase. The revenue from loading zones will increase.

For parking spaces, from $E q(5.28)$, if its allocation $\left(n_{p}\right)$ decreases, the optimal meter rate of parking spaces $\left(c_{p o}^{*}\right)$ will decrease. The revenue from parking spaces will decrease.

Based on data from Table 6.1 and 6.2 , if the number of loading zones $\left(n_{c}\right)$ increases, $c_{c o}^{*}$ will be constant while $c_{p o}^{*}$ will decrease, as shown in Figure 6.6. If $n_{c}$ 
increases, $\lambda_{c r}$ will increase but $\lambda_{p r}$ will be constant, as shown in Figure 6.7. When $n_{c}$ is 6 , the commercial revenue demand almost equals the arrival rate of commercial vehicles $\left(\lambda_{c}\right)$. If $n_{c}$ is more than 6 , all the commercial vehicles will park on street. Therefore, the maximum value of $n_{c}$ of scenario 3 is 6 .

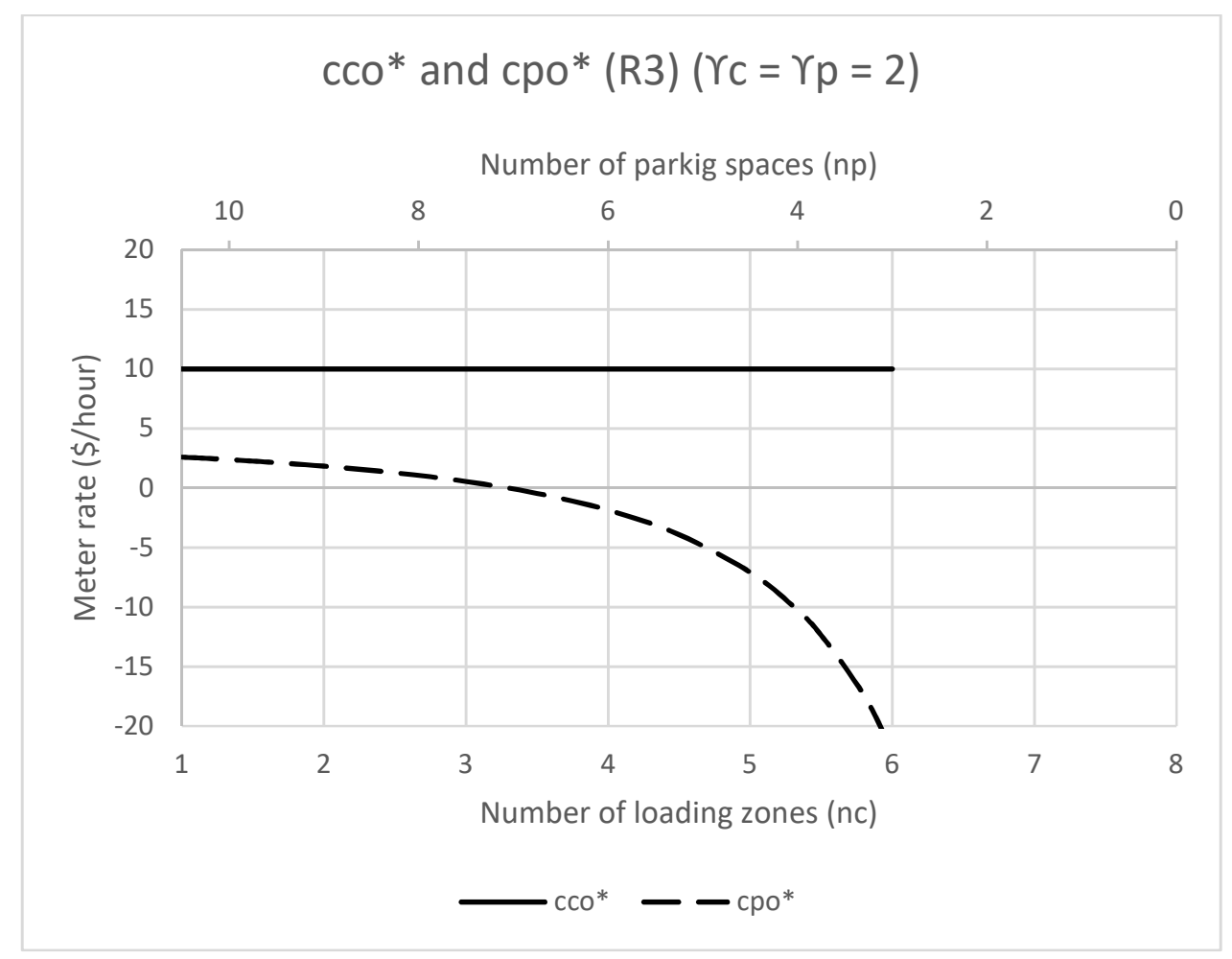

Figure 6.6 Optimal meter rates of scenario-3 revenue model 


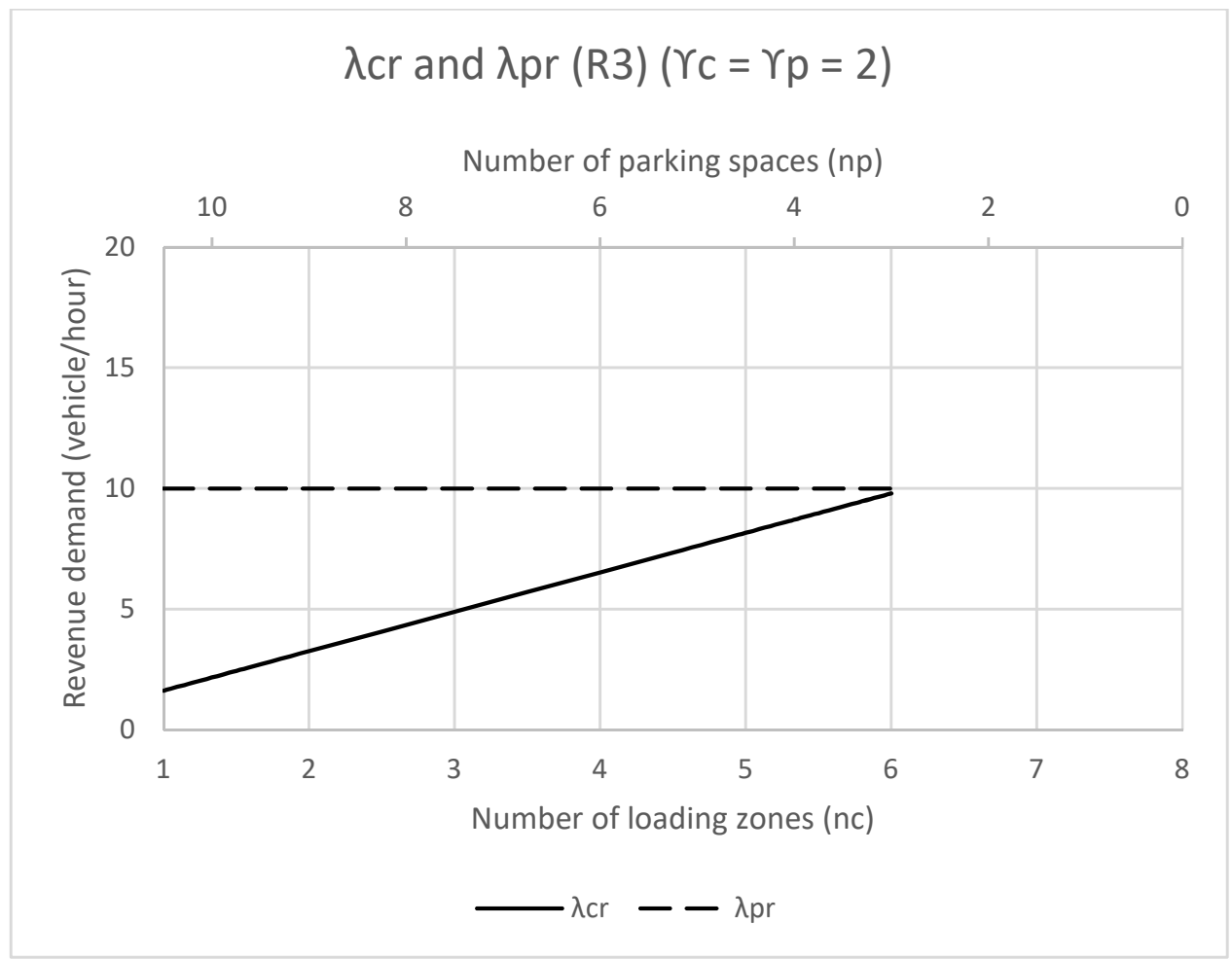

Figure 6.7 Revenue demand of scenario-3 revenue model

Since the increase in the revenue from loading zones is lower than the decrease in the revenue from parking spaces, majority of curb length is allocated to parking spaces, as shown in Figure 6.8. 


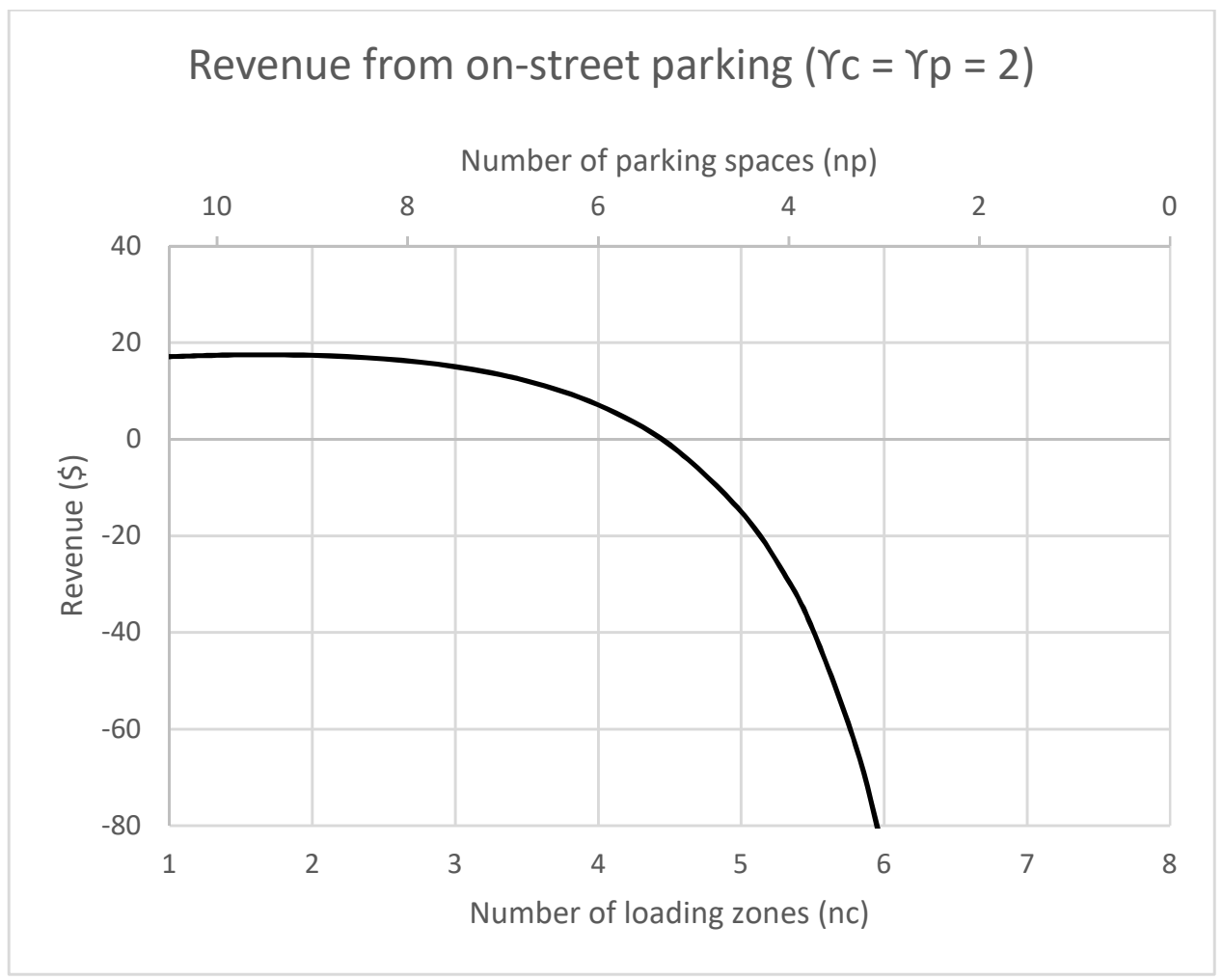

Figure 6.8 On-street scenario-3 parking revenue

\subsubsection{Optimal solution for scenario-4 revenue model}

The management strategy of scenario 4 is to let some commercial vehicles double park and to let some cars park off street. For loading zones, from $E q(5.36)$, if its curb allocation $\left(n_{c}\right)$ increases, the optimal meter rate of loading zones $\left(c_{c o}^{*}\right)$ will increase. The commercial revenue demand will increase. The revenue from loading zones will increase.

For parking spaces, from $E q(5.38)$, if its curb allocation $\left(n_{p}\right)$ decreases, the optimal meter rate of parking spaces $\left(c_{p o}^{*}\right)$ will increase. The car revenue demand will decrease. The revenue from parking spaces will decrease.

Based on data from Table 6.1 and 6.2 , if the number of loading zones $\left(n_{c}\right)$ increases, both of the optimal meter rates $\left(c_{c o}^{*}, c_{p o}^{*}\right)$ will slightly increase. $\lambda_{c r}$ will increase but $\lambda_{p r}$ will decrease, as shown in Figure 6.9. When $n_{c}$ is 6 , the commercial revenue 
demand almost equals the arrival rate of commercial vehicles $\left(\lambda_{c}\right)$. If $n_{c}$ is more than 6 , all the commercial vehicles will park on street. Therefore, the maximum value of $n_{c}$ of scenario 4 is 6.

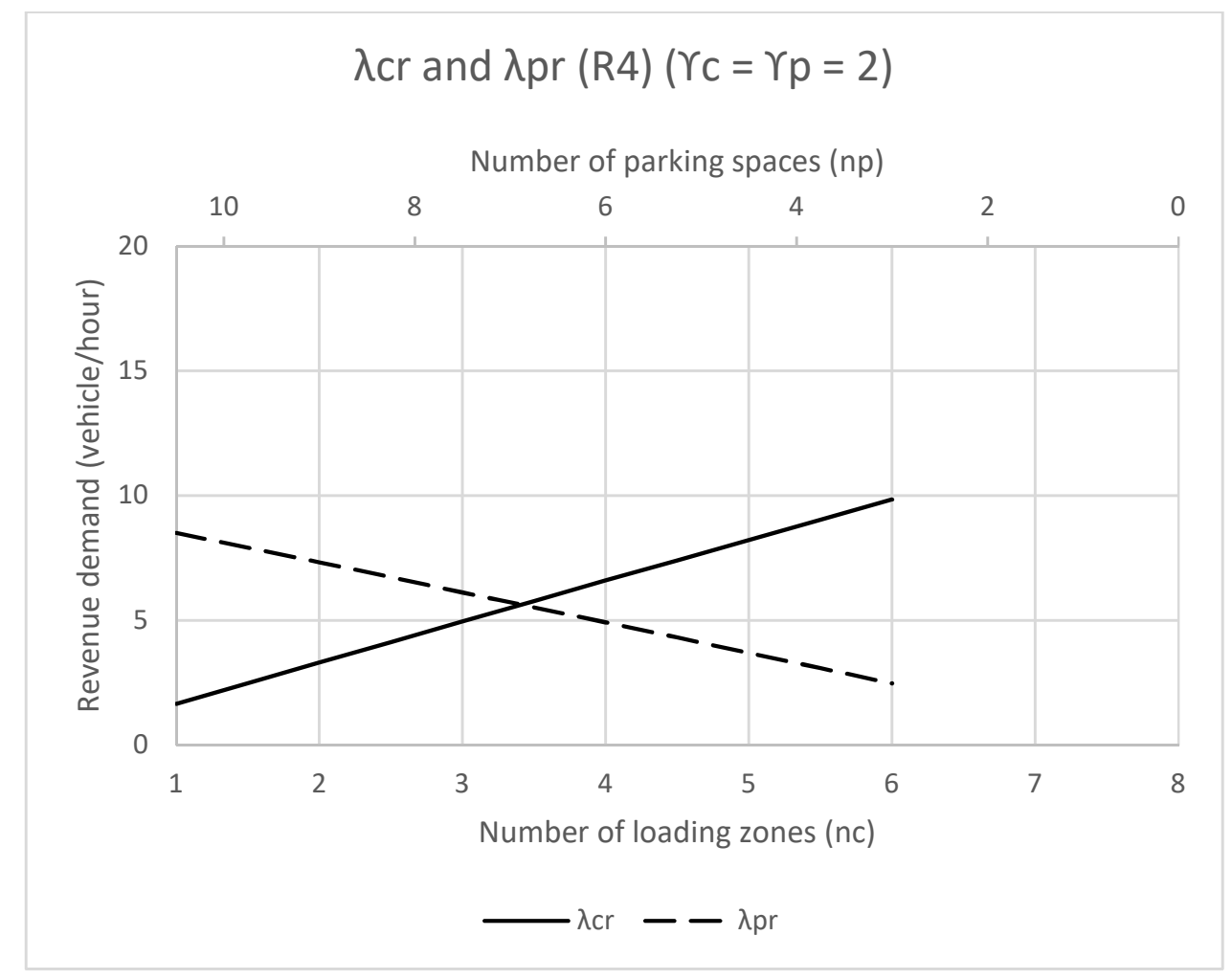

Figure 6.9 Revenue demand of scenario-4 revenue model

The increase in the commercial revenue demand is higher than the decrease in the car revenue demand. Therefore, majority of curb length is allocated to loading zones, as shown in Figure 6.10. 


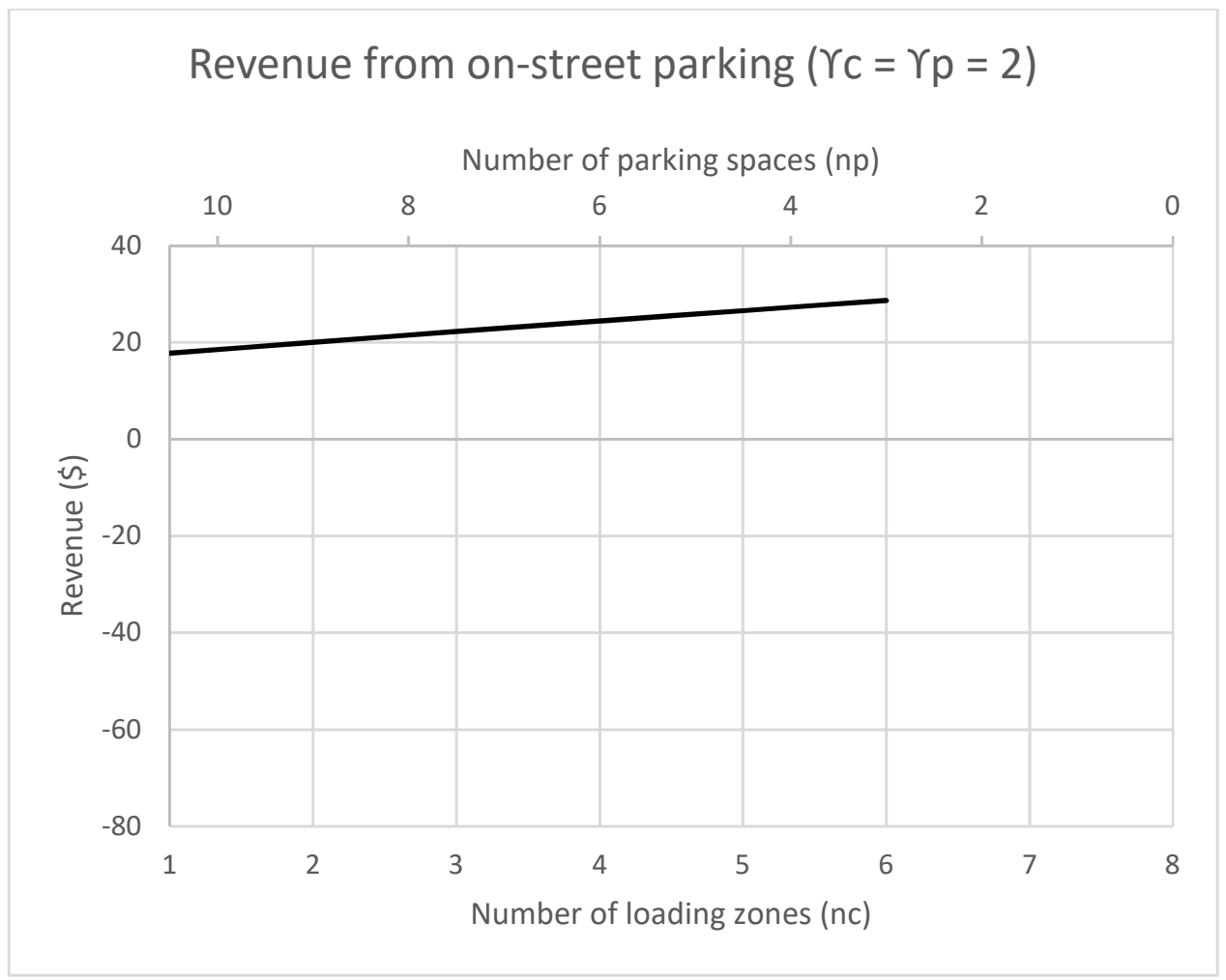

Figure 6.10 On-street scenario-4 parking revenue

\subsubsection{Optimal solution for all the revenue models}

From the optimal solutions of revenue models of all scenarios, it is found that curb length allocation, meter rates, and revenue demand are different between scenarios, as shown in Table 6.5 .

Compared with the optimal rates of parking spaces, the optimal meter rates of loading zones are very high. For commercial vehicles, the cost of the other option, double parking, is $\$ 15$. However, for cars, the cost of the other option, parking off street, is $\$ 5$. 
Table 6.5 Optimal solutions for the revenue models $\left(\gamma_{c}=\gamma_{p}=2\right)$

\begin{tabular}{|l|r|r|r|r|}
\hline Scenario & 1 & 2 & 3 & 4 \\
\hline$\lambda_{c}$ & 3 & 3 & 10 & 10 \\
\hline$\lambda_{p}$ & 10 & 20 & 10 & 20 \\
\hline$n_{c}^{*}$ & 1.8 & 2.5 & 1.5 & 6.0 \\
\hline$n_{p}^{*}$ & 9.3 & 8.3 & 9.8 & 3.0 \\
\hline$c_{c o}^{*}$ & 9.79 & 12.30 & 10.00 & 9.95 \\
\hline$\lambda_{c r}$ & 3.00 & 3.00 & 2.45 & 9.85 \\
\hline$\lambda_{c}-\lambda_{c r}$ & & & 7.55 & 0.15 \\
\hline$c_{p o}^{*}$ & 2.18 & 3.33 & 2.26 & 3.33 \\
\hline$\lambda_{p r}$ & 10.00 & 6.82 & 10.00 & 2.48 \\
\hline revenue from loading zones & 7.34 & 9.23 & 6.12 & 24.49 \\
\hline revenue from parking spaces & 10.91 & 11.37 & 11.32 & 4.13 \\
\hline total revenue & 18.25 & 20.59 & 17.44 & 28.62 \\
\hline loading zones search cost & 3.91 & 2.03 & 12.50 & 12.63 \\
\hline parking space search cost & 14.09 & 16.67 & 12.82 & 16.67 \\
\hline commercial parking maneuver cost & 1.95 & 1.95 & 1.59 & 6.40 \\
\hline car parking maneuver cost & 1.30 & 0.89 & 1.30 & 0.32 \\
\hline double parking cost & 0.00 & 1.00 & 54.92 & 1.10 \\
\hline total social value & -2.99 & -1.93 & -65.69 & -8.49 \\
\hline
\end{tabular}

Compared with scenario 1 , the number of parking spaces of scenario 2 is lower although the passenger car arrival rate of scenario 2 is higher. For scenario 2, car drivers have multiple parking options while, for scenario 1, car drivers have only one parking option. For scenario 2, if parking spaces are congested, some car drivers will just park off street. The parking space meter rate of scenario 2 is not as low as the parking space meter rate of scenario 1 . The parking space meter rate of scenario 1 is low in order to keep the on-street option cheaper than the off-street option.

Compared with scenario 1, the number of loading zones of scenario 3 is lower although the commercial vehicle arrival rate of scenario 3 is higher. For scenario 3 , commercial drivers have multiple parking options while, for scenario 1 , they have only one parking option. For scenario 3, if loading zones are congested, some commercial drivers 
will just double park. The loading zone meter rate of scenario 2 is not as low as the loading zone meter rate of scenario 1 . The loading zone meter rate of scenario 1 is low in order to keep the on-street option cheaper than the double-parking option.

Compared with scenario 3, scenario 4 makes more revenue. Scenario 4 gives more options for both commercial vehicles and passenger cars. For each parking type, if its arrival rate is high, some drivers will choose the other option in order to avoid high parking search cost and the optimal rate will not be too low. For scenario 4, majority of curb length is allocated to a parking type that makes the most revenue. However, for scenario 3 , majority of curb length to allocate to parking spaces to prevent high parking search cost.

\subsection{Impact of exponents of the parking search time function}

The parking search costs of both commercial vehicles and passenger cars (Section 4.3) are expressed in Table 6.6. The parking search cost is a function of parking utilization to the power of $\gamma_{c}$ or $\gamma_{p}$. In this study, the $\gamma_{c}$ and $\gamma_{p}$ have two values: 1 and 2. If the value is 1 , the parking search cost is a linear function of parking utilization. The value of 1 gives better understanding of a mathematical relationship and is easier to use in a numerical experiment that can be observed in Table 5.1. The value of 2 gives better estimates for the parking search cost which results in more accurate objective values. From literature, the parking search cost is not a linear function of parking utilization (Lam et al., 2006; MillardBall et al., 2014; Zheng and Geroliminis, 2016). 
Table 6.6 Estimates of parking search cost

\begin{tabular}{|l|c|}
\hline \multicolumn{1}{|c|}{ Parking search cost } & Formula \\
\hline Commercial vehicles & $c_{c t} \alpha_{w c}\left(\frac{\lambda_{c}}{n_{c} \mu_{c}}\right)^{\gamma_{c}}$ \\
\hline Passenger cars & $c_{p t}(1+\vartheta) \alpha_{w p}\left(\frac{\lambda_{p}}{n_{p} \mu_{p}}\right)^{\gamma_{p}}$ \\
\hline
\end{tabular}

If the parking utilization, $\frac{\lambda_{c}}{n_{c} \mu_{c}}$ and $\frac{\lambda_{p}}{n_{p} \mu_{p}}$ are less than one, the higher the exponents $\left(\gamma_{c}\right.$ and $\left.\gamma_{p}\right)$, the lower the parking search cost, as shown in Figure 6.11. The total on-street cost is assumed to be constant. The higher the exponents, the higher the parking monetary cost. The parking monetary cost equals the parking revenue. Therefore, the higher the exponents, the higher the revenue. However, if the parking utilization factors are more than one, the higher the exponents, the higher the parking search cost, the lower the parking monetary cost, and the lower the revenue.

\section{Parking search cost}

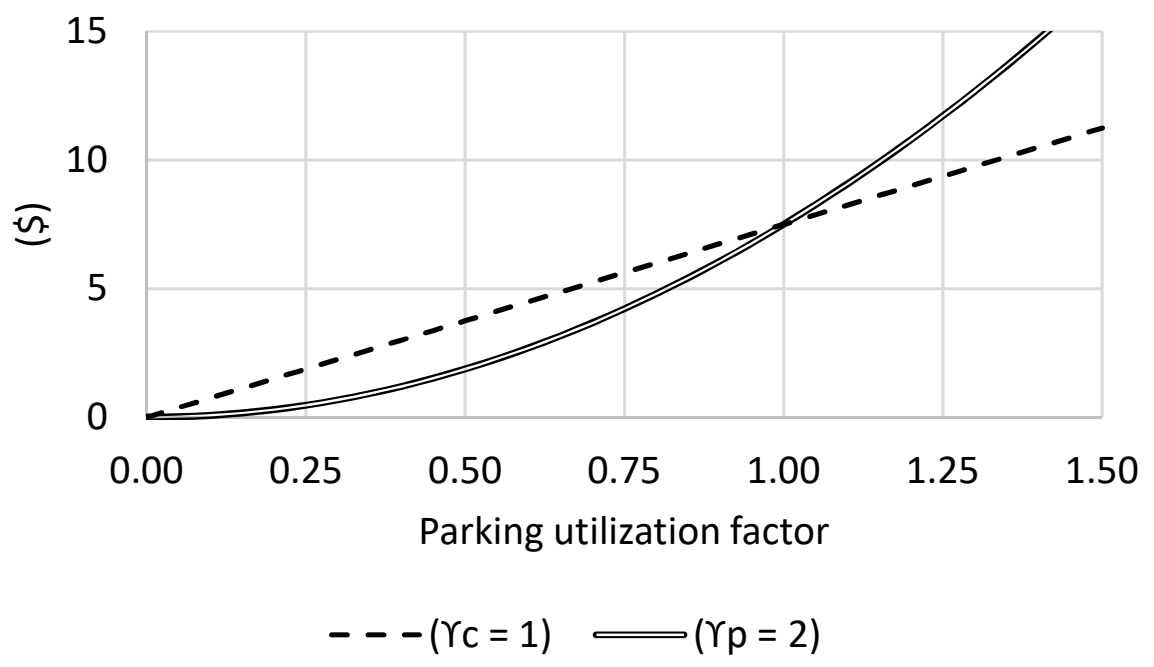

Figure 6.11 Impact of exponents on parking search cost 
From Chapter 5, the parking revenue is expressed as follow, $y_{r e v}=\frac{c_{c o} \lambda_{c r}}{\mu_{c}}+\frac{c_{p o} \lambda_{p r}}{\mu_{p}}$

From Section 5.7, the optimal meter rates are different between scenarios. In addition, from Section 4.5, the revenue demand $\lambda_{c r}$ and $\lambda_{p r}$ is a function of the meter rates. This leads to different estimates in the parking revenue between scenarios. It is required to analyze the impact of the exponents on the parking revenue for one scenario at a time.

\section{$\underline{6.4 .1 \text { Impact of exponents on scenario-1 revenue }}$}

According to optimal solution of scenario 1 in Table 6.5 , majority of curb length is allocated to parking spaces. From Section 6.2, the revenue is highly related to the meter rate of parking spaces $\left(c_{p o}\right)$. According to Section 5.7, the parking space meter rates of scenario 1 are expressed below,

$c_{p o}^{*}(R 1)=c_{p f}+c_{p f f} \mu_{p}-c_{p t}(1+\vartheta) \alpha_{w p} \mu_{p}\left(\frac{\lambda_{p}}{n_{p} \mu_{p}}\right)^{\gamma_{p}}$

From the formula above, if parking utilization of parking spaces $\left(\frac{\lambda_{p}}{n_{p} \mu_{p}}\right)$ is less than one, the higher the exponent, the higher the optimal meter rate, the higher the revenue. However, if the parking utilization is more than one, the higher the exponent, the lower the optimal meter rate, the lower the revenue, as shown in Figure 6.12. 


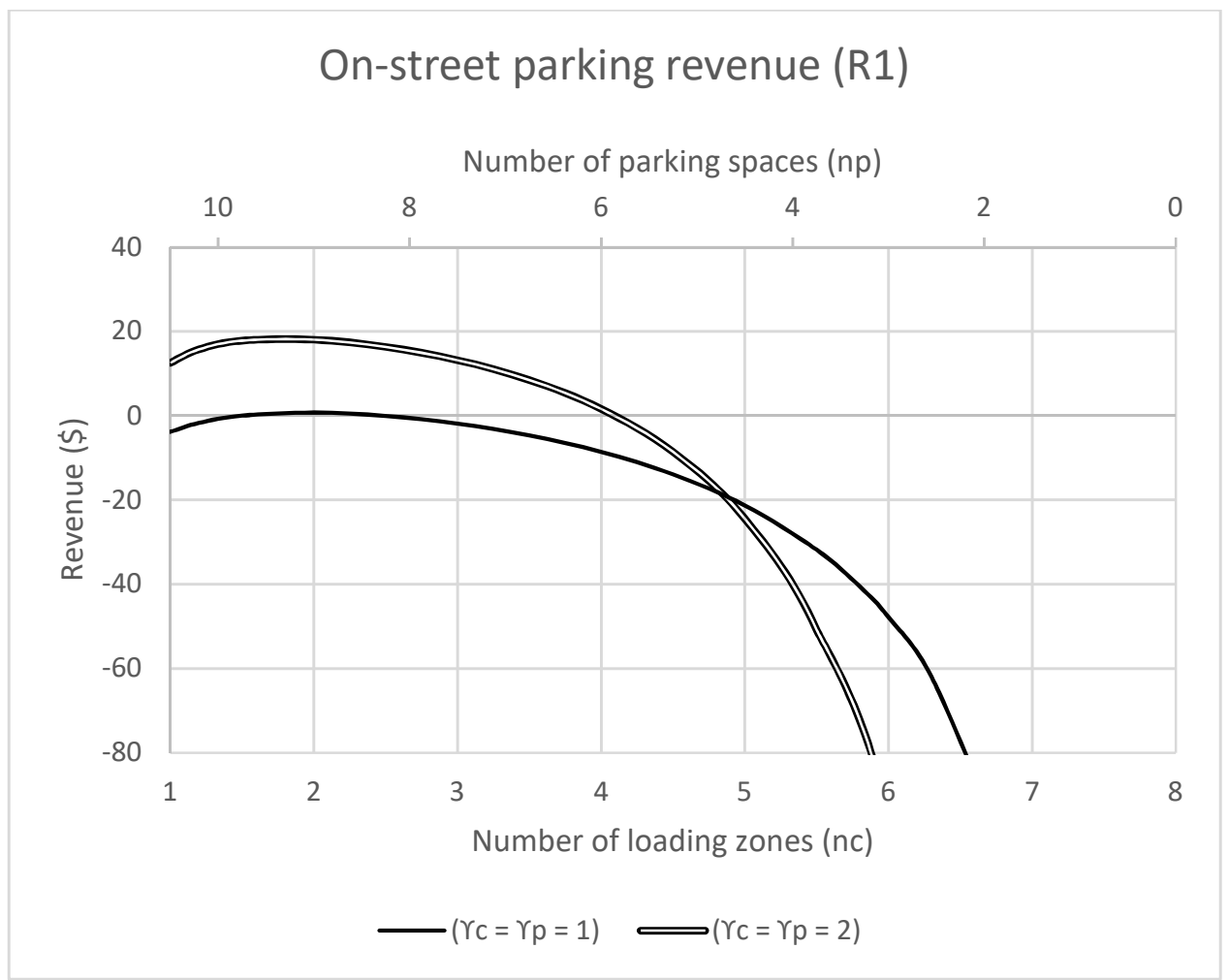

Figure 6.12 Impact of exponents on the revenue for scenario 1

\subsubsection{Impact of exponents on scenario-2 revenue}

According to optimal solution of scenario 2 in Table 6.5, majority of curb length is allocated to parking spaces. From Section 6.2, the revenue is highly related to the meter rate of parking spaces $\left(c_{p o}\right)$. According to Section 5.7, the parking space meter rates of scenario 2 are expressed as follow,

$c_{p o}^{*}(R 2)=\frac{\gamma_{p}\left(c_{p f}+c_{p f f} \mu_{p}\right)}{\left(\gamma_{p}+1\right)}$

From the formula above, the higher the exponent, the higher the meter rate, the higher the revenue, as shown in Figure 6.13. From Section 6.2.2, the more the number of loading zones $\left(n_{c}\right)$, the less the car revenue demand $\left(\lambda_{p r}\right)$, the less the difference between the revenue of $\left(\gamma_{c}=\gamma_{p}=2\right)$ and the revenue of $\left(\gamma_{c}=\gamma_{p}=1\right)$. 


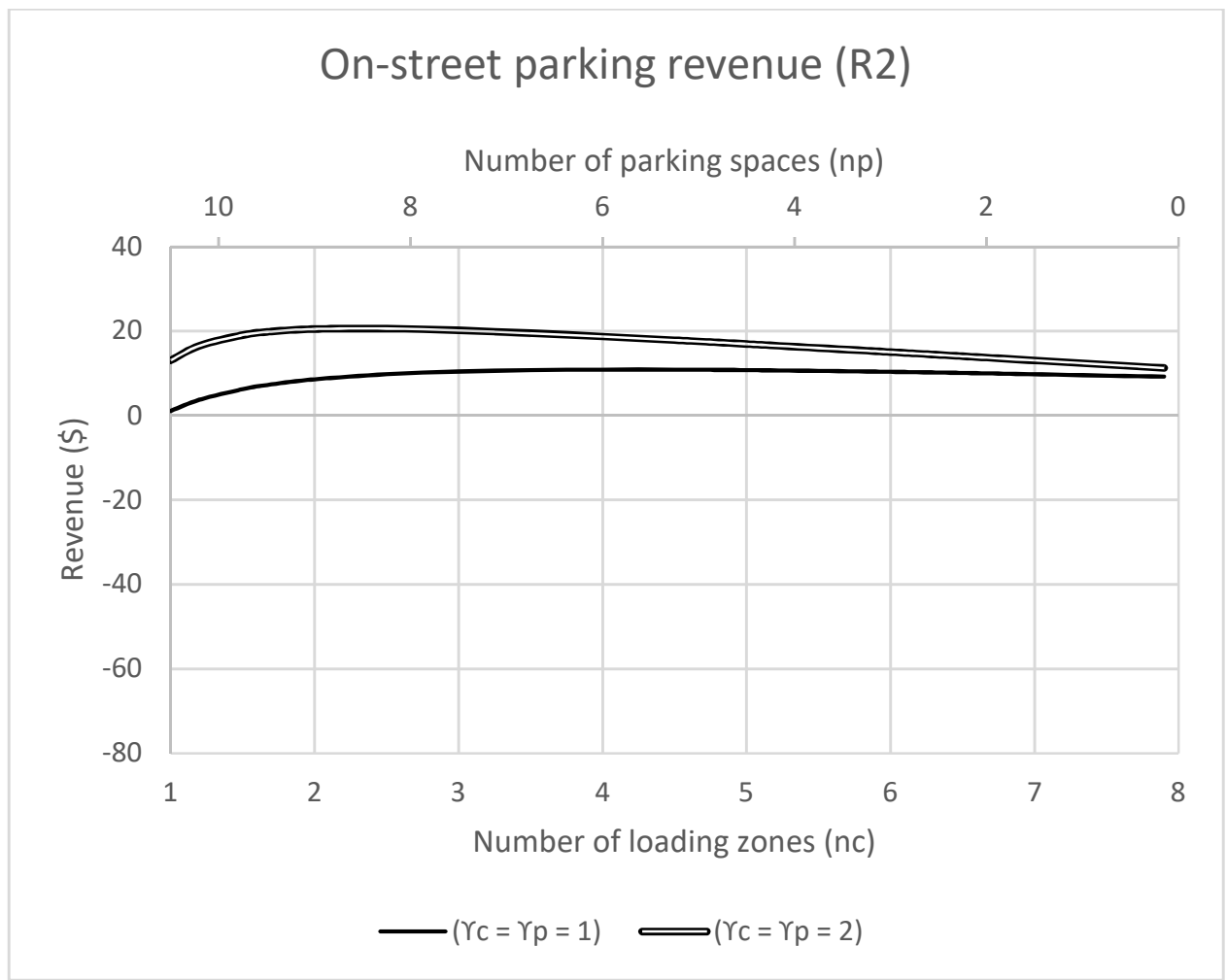

Figure 6.13 Impact of exponents on the revenue for scenario 2

\subsubsection{Impact of exponents on scenario-3 revenue}

According to optimal solution of scenario 3 in Table 6.5, majority of curb length is allocated to parking spaces. From Section 6.2 , the revenue is highly related to the meter rate of parking spaces $\left(c_{p o}\right)$. According to Section 5.7, the parking space meter rates of scenario 3 are expressed as follow,

$c_{p o}^{*}(R 3)=c_{p f}+c_{p f f} \mu_{p}-c_{p t}(1+\vartheta) \alpha_{w p} \mu_{p}\left(\frac{\lambda_{p}}{n_{p} \mu_{p}}\right)^{\gamma_{p}}-c_{p t} \mu_{p} f_{d b} t_{s}\left(\lambda_{c}-\lambda_{c}^{*}\right)$

From the formula above, if parking utilization of parking spaces $\left(\frac{\lambda_{p}}{n_{p} \mu_{p}}\right)$ is less than one, the higher the exponent, the higher the optimal meter rate, the higher the revenue. However, if the parking utilization is more than one, the higher the exponent, the lower the optimal meter rate, the lower the revenue, as shown in Figure 6.14. 


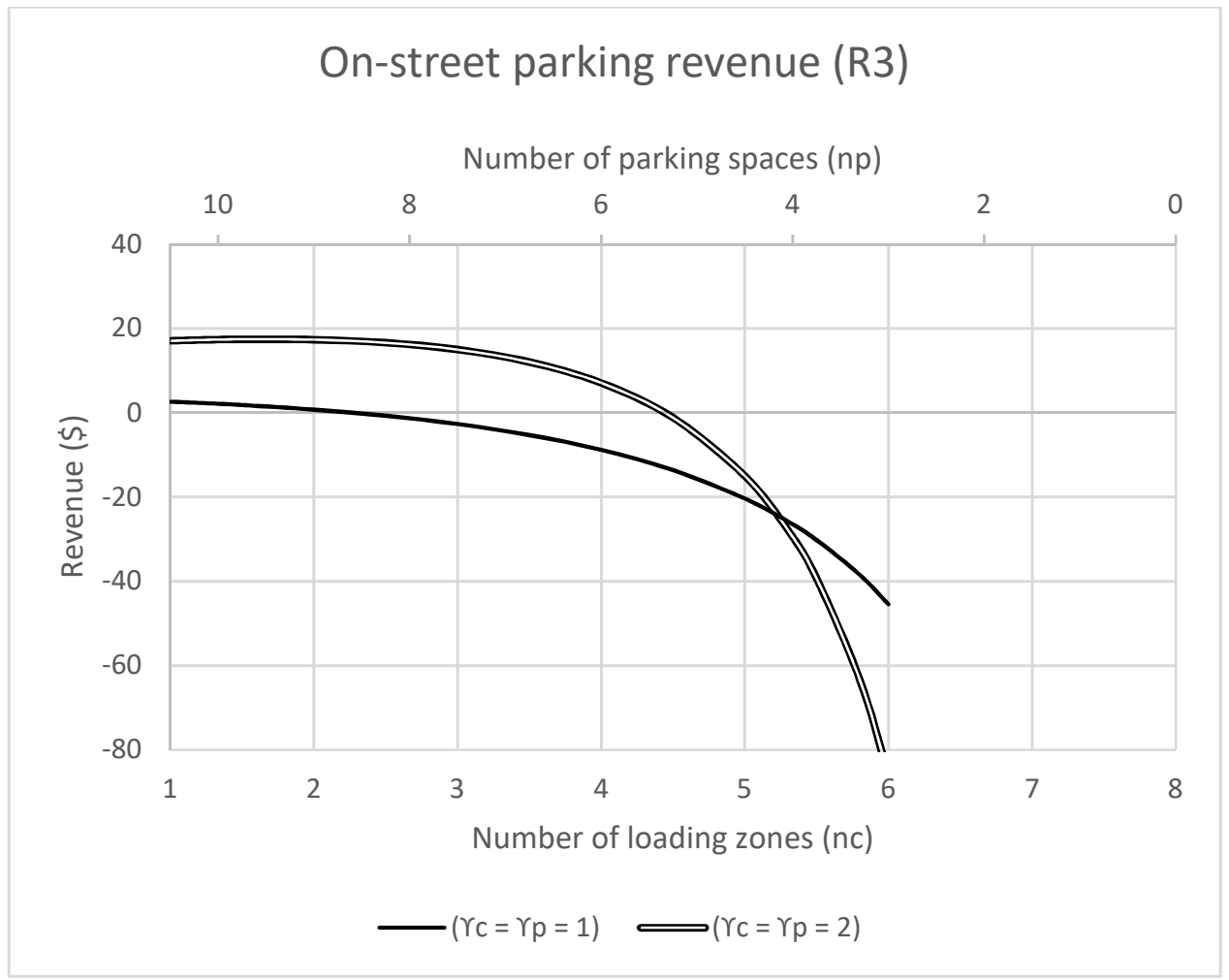

Figure 6.14 Impact of exponents on the revenue for scenario 3

\subsubsection{Impact of exponents on scenario-4 revenue}

According to Section 5.7, the formula of the optimal meter rates of scenario 4 are complicated. It is much easier to show the numerical results of the meter rates which are shown in Figure 6.15. The optimal meter rates of $\left(\gamma_{c}=\gamma_{p}=2\right)$ is higher than the optimal meter rates of $\left(\gamma_{c}=\gamma_{p}=1\right)$. This leads to higher revenue, as shown in Figure 6.16. 


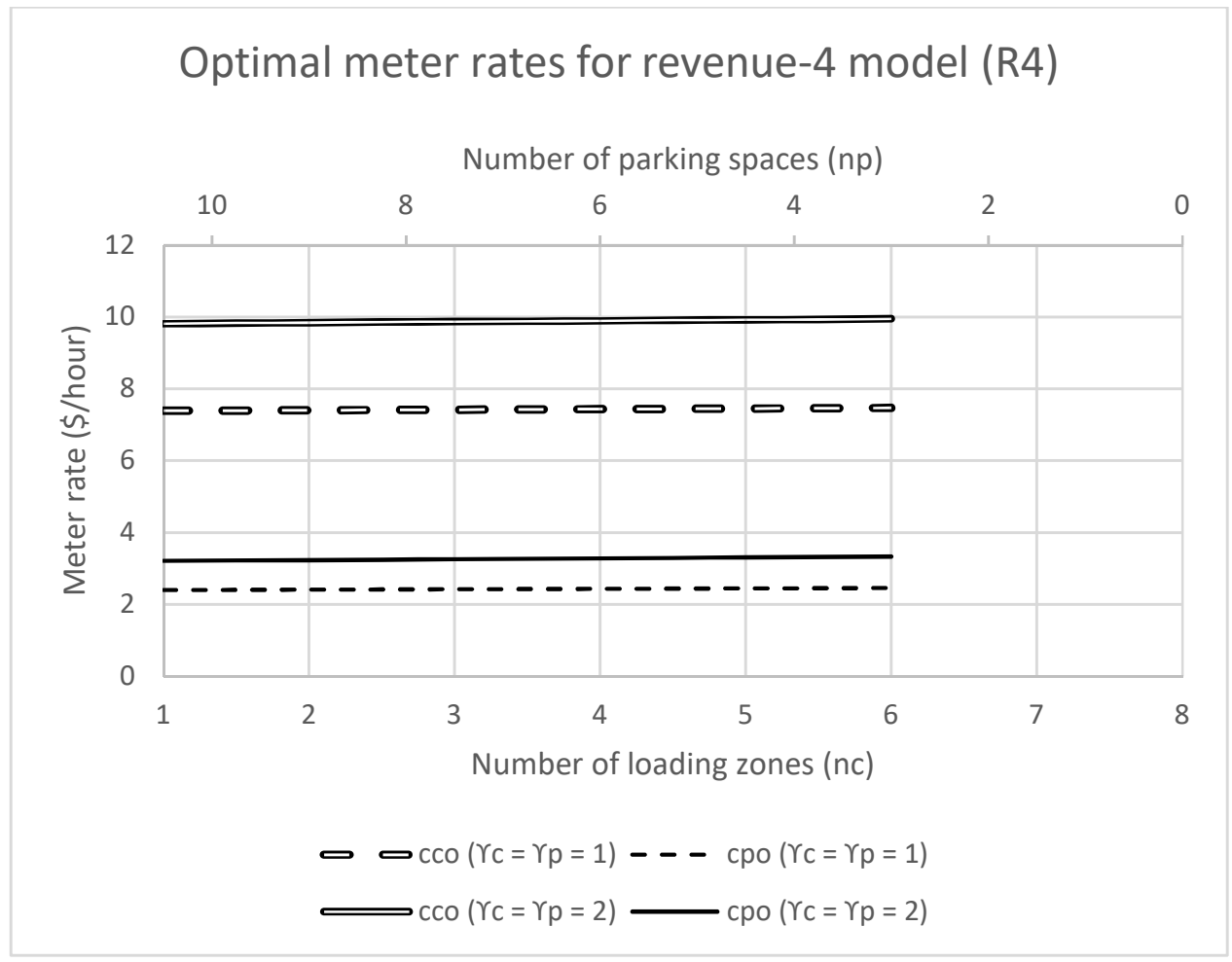

Figure 6.15 Optimal rates for scenario-4 revenue models with different exponents

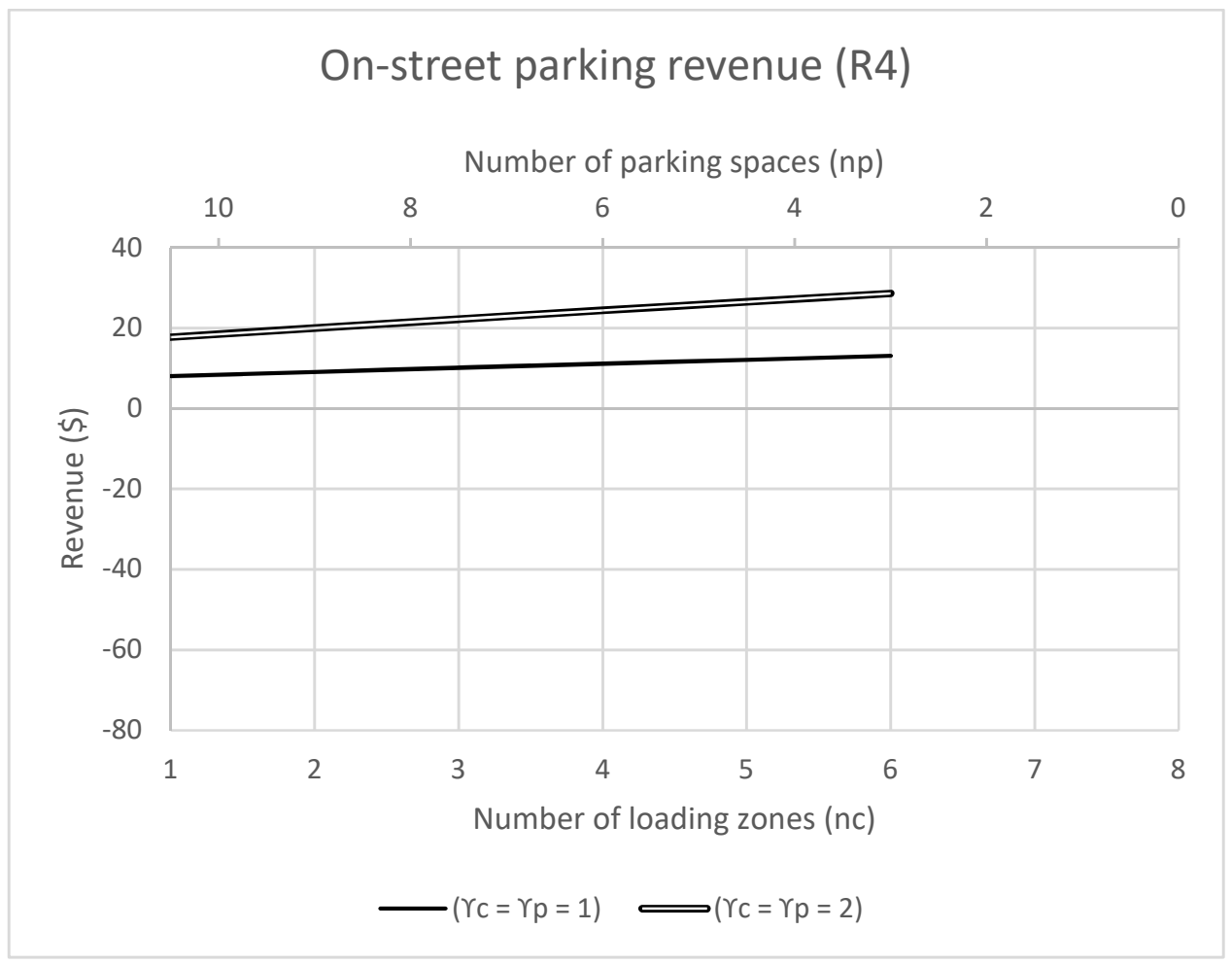

Figure 6.16 Impact of exponents on the revenue for scenario 4 100 


\subsection{Conclusion}

This chapter demonstrates how the revenue maximization models optimize meter rates and curb length allocation. The revenue models are developed based on four scenarios. The main difference between scenarios is how many parking options are chosen. For some scenarios, only the on-street option is chosen; for other scenarios, two options are chosen - parking on street and other option.

Each scenario has its own data set. Most of the data is the same among the scenarios. The differences are arrival rates of commercial vehicles and passenger cars. The arrival rates coincide with parking options. The more the arrival rates, the more the parking options are chosen.

Before analyzing a case study for each scenario, the arrival rates of commercial vehicles and passenger cars is changed into the aggregated arrival rate of passenger vehicle equivalent, in terms of unit parking length and parking duration. Without considering vehicle types, it is very clear to analyze relationship between the aggregated arrival rate and the meter rate. If the arrival rate is low, the meter rate will change, responding to change in the aggregated arrival rate. If the arrival rate is high, it will be practical to keep the meter rate the same and some drivers will choose other options, rather than all drivers choosing the on-street option.

Of scenario 1 (parking on street only), the revenue demand (how many vehicles park on street) is constant. The meter rates of loading zones and parking spaces are positively related to curb length allocation. The less the curb allocation, the lower the meter rate, the lower the revenue. Majority of curb length is allocated to a parking type of which meter rate is more positively related to the allocation. 
Compared with scenario 2 (on-street and off-street are chosen), the revenue of scenario 1 is lower. For scenario 2, if parking spaces are congested, some car drivers will just park off street. The meter rate of scenario 2 is higher than the meter rate of scenario 1 . The meter rate of scenario 1 is low in order to keep the on-street option cheaper than the off-street option.

Of scenario 4 (all parking options chosen), meter rates of loading zones and parking spaces are slightly related to curb length allocation. For each parking type, its revenue demand is positively related to its curb allocation. The more the curb allocation, the more the revenue demand, the more the revenue. Majority of curb length is allocated to loading zones of which revenue demand is more positively related to curb allocation.

Compared with scenario 4, the revenue of scenario 3 (on-street and double-parking are chosen) is lower. For scenario 4, if parking spaces are congested, some car drivers will just park off street. However, for scenario 3, all car drivers will park on street only. The parking space meter rate of scenario 4 is higher than the parking space meter rate of scenario 3 . The parking space meter rate of scenario 3 is low in order to keep the on-street option cheaper than the off-street option.

The exponents of the parking search time function affect the revenue. The higher the exponents, the higher the revenue. There is one exception where all the vehicles park on street only. If parking utilization is high, the optimal meter rate of the higher exponent will be less than the optimal meter rate of the lower exponent. This leads to the revenue of higher exponent less than the revenue of lower exponent. 


\section{Chapter 7 Social Optimum Optimization Models}

This chapter proposes an optimization model maximizing total social optimum (SOC) of the on-street parking system. The main sections of this chapter are the definition of SOC optimization models, model formulation, optimal solutions for the four scenarios described in Section 4.6, and solution interpretation.

\subsection{Definition of social optimum optimization models}

The social optimum (SOC) optimization models are to maximize total SOC of the on-street parking system by finding optimal values of decision variables. The SOC equals revenues minus costs. The revenues are on-street parking fees from loading zones and parking spaces. The costs include parking search cost, parking maneuver cost, and traffic delay cost due to double parking.

The decision variables are the commercial vehicle parking meter rate $\left(c_{c o}\right)$, the

passenger vehicle on-street parking meter rate $\left(c_{p o}\right)$, and the number of parking spaces $\left(n_{p}\right)$. The number of loading zones $\left(n_{c}\right)$ is calculated using the relationship between $n_{c}$ and $n_{p}$ in Section 4.4.

\subsection{Model formulation}

The revenues and the costs are already explained and formulated in Section 4.3. Therefore, this section only formulates the objective function.

The objective of the model is to maximize the social optimum (SOC) of the onstreet parking system, which is expressed as follows, 


$$
\begin{aligned}
y_{s o c}=\frac{c_{c o} \lambda_{c r}}{\mu_{c}} & +\frac{c_{p o} \lambda_{p r}}{\mu_{p}}-c_{c t} \alpha_{w c}\left(\frac{\lambda_{c r}}{n_{c} \mu_{c}}\right)^{\gamma_{c}} \lambda_{c}-c_{d c} \lambda_{c r}-c_{d b}\left(\lambda_{c}-\lambda_{c r}\right) \quad E q(7.1) \\
& -\left[c_{p t}(1+\vartheta) \alpha_{w p}\left(\frac{\lambda_{p r}}{n_{p} \mu_{p}}\right)^{\gamma_{p}}+c_{p t} f_{d b} t_{s}\left(\lambda_{c}-\lambda_{c r}\right)\right] \lambda_{p}-c_{d p} \lambda_{p r}
\end{aligned}
$$

\subsection{SOC model for Scenario 1: All vehicles parking on street}

Of the model for Scenario 1, its constraints are already explained in Section 5.3.

Then, the next step is to take the objective function into account to find the optimal solutions.

\subsubsection{Objective function for Scenario 1}

All vehicles park on street. The revenue demand equals the arrival rates of parking vehicles, as shown below,

$\lambda_{c r}=\lambda_{c}$

$\lambda_{p r}=\lambda_{p}$

Then, the social-optimum estimation, $E q(7.1)$, becomes,

$$
\begin{gathered}
y_{s o c}(1)=\frac{c_{c o} \lambda_{c}}{\mu_{c}}+\frac{c_{p o} \lambda_{p}}{\mu_{p}}-c_{c t} \alpha_{w c}\left(\frac{\lambda_{c}}{n_{c} \mu_{c}}\right)^{\gamma_{c}} \lambda_{c}-c_{d c} \lambda_{c} \\
-c_{p t}(1+\vartheta) \alpha_{w p}\left(\frac{\lambda_{p}}{n_{p} \mu_{p}}\right)^{\gamma_{p}} \lambda_{p}-c_{d p} \lambda_{p}
\end{gathered}
$$

\subsubsection{Optimal value of $c_{c a}$}

Since the social optimum (SOC) is a linear function of $c_{c o}$, the optimal $c_{c o}$ is one of extreme points of a feasible region. $E q(5.3)$ gives the feasible region. The optimal $c_{c o}$ is expressed below, 
$c_{c o}^{*}(S 1)=\frac{c_{v} n_{e}}{\tau_{e}}-c_{c t} \alpha_{w c} \mu_{c}\left(\frac{\lambda_{c}}{n_{c} \mu_{c}}\right)^{\gamma_{c}}$

\subsubsection{Optimal value of $c_{p o}$}

Since the social optimum (SOC) is a linear function of $c_{p o}$, the optimal $c_{p o}$ is one of extreme points of a feasible region. $E q(5.6)$ gives the feasible region. The optimal $c_{p o}$ is expressed below,

$$
c_{p o}^{*}(S 1)=c_{p f}+c_{p f f} \mu_{p}-c_{p t}(1+\vartheta) \alpha_{w p} \mu_{p}\left(\frac{\lambda_{p}}{n_{p} \mu_{p}}\right)^{\gamma_{p}}
$$

\subsubsection{Optimal value of $n_{c}$ and $n_{p}$}

To reduce the model complication, both $\gamma_{c}$ and $\gamma_{p}$ are assumed to be 1.0 , the social optimum (SOC) becomes,

$$
\begin{gathered}
y_{s o c}\left(1 \mid \gamma_{c}=\gamma_{p}=1\right)=\frac{c_{c o} \lambda_{c}}{\mu_{c}}+\frac{c_{p o} \lambda_{p}}{\mu_{p}}-c_{c t} \alpha_{w c}\left(\frac{\lambda_{c}}{n_{c} \mu_{c}}\right) \lambda_{c}-c_{d c} \lambda_{c} \quad E q(7.5) \\
-c_{p t}(1+\vartheta) \alpha_{w p}\left(\frac{\lambda_{p}}{n_{p} \mu_{p}}\right) \lambda_{p}-c_{d p} \lambda_{p}
\end{gathered}
$$

The SOC is an unconstrained non-linear function of $n_{p} . n_{p}$ is optimized based on the necessary condition,

$$
\frac{\partial y_{s o c}}{\partial n_{p}}=-c_{c t} \alpha_{w c}\left(\frac{\lambda_{c}^{2}}{\mu_{c}}\right) \frac{l_{c} l_{p}}{\left(l_{b}-n_{p} l_{p}\right)^{2}}+c_{p t}(1+\vartheta) \alpha_{w p}\left(\frac{\lambda_{p}^{2}}{\mu_{p}}\right) \frac{1}{n_{p}^{2}}=0
$$

From the equation above, the optimal $n_{p}$ is expressed below,

$$
n_{p}^{*}\left(S 1 \mid \gamma_{c}=\gamma_{p}=1\right)=\frac{l_{b}}{l_{p}+\left(\frac{\lambda_{c}}{\lambda_{p}}\right) \sqrt{l_{c} l_{p} \frac{c_{c t} \alpha_{w c}}{c_{p t}(1+\vartheta) \alpha_{w p}}\left(\frac{\mu_{p}}{\mu_{c}}\right)}}
$$


The optimality condition is checked with the sufficient condition,

$$
\frac{\partial^{2} y_{s o c}\left(\gamma_{c}=\gamma_{p}=1\right)}{\partial n_{p}^{2}}=-2 c_{c t} \alpha_{w c}\left(\frac{\lambda_{c}^{2}}{\mu_{c}}\right) \frac{l_{c} l_{p}^{2}}{\left(l_{b}-n_{p} l_{p}\right)^{3}}-2 c_{p t}(1+\vartheta) \alpha_{w p}\left(\frac{\lambda_{p}^{2}}{\mu_{p}}\right) \frac{1}{n_{p}^{3}}<0
$$

The solution is a local maximum.

\subsection{SOC model for Scenario 2: Some passenger cars park off street}

Of the model for Scenario 2, its constraints are already explained in Section 5.4. Then, the next step is to take the objective function into account to find the optimal solutions.

\subsubsection{Objective function for Scenario 2}

All commercial vehicles park on street. The commercial revenue demand $\left(\lambda_{c r}\right)$ equals the arrival rates of commercial parking vehicles $\left(\lambda_{c}\right)$. However, some passenger cars park off street. The car revenue demand $\left(\lambda_{p r}\right)$ equals the car breakeven demand $\left(\lambda_{p}^{*}\right)$, as shown below,

$\lambda_{c r}=\lambda_{c}$

$\lambda_{p r}=\lambda_{p}^{*}$

Then, the social-optimum function, $E q(7.1)$, becomes,

$$
\begin{gathered}
y_{s o c}(2)=\frac{c_{c o} \lambda_{c}}{\mu_{c}}+\frac{c_{p o} \lambda_{p}^{*}}{\mu_{p}}-c_{c t} \alpha_{w c}\left(\frac{\lambda_{c}}{n_{c} \mu_{c}}\right)^{\gamma_{c}} \lambda_{c}-c_{d c} \lambda_{c} \quad E q(7.7) \\
-c_{p t}(1+\vartheta) \alpha_{w p}\left(\frac{\lambda_{p}^{*}}{n_{p} \mu_{p}}\right)^{\gamma_{p}} \lambda_{p}-c_{d p} \lambda_{p}^{*}
\end{gathered}
$$

The car breakeven demand $\left(\lambda_{p}^{*}\right)$ is estimated with $E q(5.16)$. Then, the socialoptimum function becomes, 


$$
\begin{array}{rl}
y_{s o c}(2)=\frac{c_{c o} \lambda_{c}}{\mu_{c}}-c_{c t} \alpha_{w c}\left(\frac{\lambda_{c}}{n_{c} \mu_{c}}\right)^{\gamma_{c}} \lambda_{c}-c_{d c} \lambda_{c} & E q(7.8) \\
+ & \frac{n_{p}\left(c_{p o}-c_{d p} \mu_{p}\right)\left(c_{p f}+c_{p f f} \mu_{p}-c_{p o}\right)^{\frac{1}{\gamma_{p}}}}{\left[c_{p t}(1+\vartheta) \alpha_{w p} \mu_{p}\right]^{\frac{1}{\gamma_{p}}}}-\left(c_{p f}+c_{p f f} \mu_{p}-c_{p o}\right) \frac{\lambda_{p}}{\mu_{p}}
\end{array}
$$

\subsubsection{Optimal value of $c_{c a}$}

Since the social optimum (SOC) is a linear function of $c_{c o}$, the optimal $c_{c o}$ is one of extreme points of a feasible region. $E q(5.13)$ gives the feasible region. The optimal $c_{c o}$ is expressed below,

$$
c_{c o}^{*}(S 2)=\frac{c_{v} n_{e}}{\tau_{e}}-c_{c t} \alpha_{w c} \mu_{c}\left(\frac{\lambda_{c}}{n_{c} \mu_{c}}\right)^{\gamma_{c}}
$$

\subsubsection{Optimal value of $c_{p e}$}

The social optimum is an unconstrained non-linear function of $c_{p o} . c_{p o}$ is optimized based on the necessary condition,

$$
\frac{\partial y_{s o c}}{\partial c_{p o}}=\frac{n_{p}\left[\gamma_{p}\left(c_{p f}+c_{p f f} \mu_{p}-c_{p o}\right)-\left(c_{p o}-c_{d p} \mu_{p}\right)\right]}{\gamma_{p}\left[c_{p t}(1+\vartheta) \alpha_{w p} \mu_{p}\right]^{\frac{1}{\gamma_{p}}}\left(c_{p f}+c_{p f f} \mu_{p}-c_{p o}\right)^{\frac{\gamma_{p}-1}{\gamma_{p}}}}+\frac{\lambda_{p}}{\mu_{p}}=0
$$

From the equation above, the optimal $c_{p o}$ is expressed below,

$$
\begin{aligned}
& \frac{n_{p}\left[\gamma_{p}\left(c_{p f}+c_{p f f} \mu_{p}\right)-c_{p o}^{*}(S 2)\left(\gamma_{p}+1\right)+c_{d p} \mu_{p}\right]}{\left[c_{p t}(1+\vartheta) \alpha_{w p} \mu_{p}\right]^{\frac{1}{\gamma_{p}}} \gamma_{p}\left(c_{p f}+c_{p f f} \mu_{p}-c_{p o}^{*}(S 2)\right)^{\frac{\gamma_{p}-1}{\gamma_{p}}}}+\frac{\lambda_{p}}{\mu_{p}}=0 \\
& \text { If } \gamma_{p}=1, \\
& c_{p o}^{*}\left(S 2 \mid \gamma_{p}=1\right)=\frac{c_{p f}+c_{p f f} \mu_{p}}{2}+\frac{c_{d p} \mu_{p}}{2}+\frac{c_{p t}(1+\vartheta) \alpha_{w p}}{2}\left(\frac{\lambda_{p}}{n_{p}}\right)
\end{aligned}
$$


The optimality condition is checked with the sufficient condition,

$$
\frac{\partial^{2} y_{s o c}}{\partial c_{p o}^{2}}=-\frac{n_{p}\left[2 \gamma_{p}\left(c_{p f}+c_{p f f} \mu_{p}\right)-\left(\gamma_{p}+1\right) c_{p o}-\left(\gamma_{p}-1\right) c_{d p} \mu_{p}\right]}{\gamma_{p}^{2}\left[c_{p t}(1+\vartheta) \alpha_{w p} \mu_{p}\right]^{\frac{1}{\gamma_{p}}}\left(c_{p f}+c_{p f f} \mu_{p}-c_{p o}\right)^{\frac{2 \gamma_{p}-1}{\gamma_{p}}}}<0
$$

Therefore, the solution is a local maximum.

\subsubsection{Optimal value of $n_{c}$ and $n_{p}$}

To reduce the model complication, both $\gamma_{c}$ and $\gamma_{p}$ are assumed to be 1.0 , the social optimum (SOC) is expressed below,

$$
\begin{aligned}
y_{s o c}\left(2 \mid \gamma_{c}=\gamma_{p}\right. & =1)=\frac{c_{c o} \lambda_{c}}{\mu_{c}}-c_{c t} \alpha_{w c}\left(\frac{\lambda_{c}}{n_{c} \mu_{c}}\right) \lambda_{c}-c_{d c} \lambda_{c} \quad E q(7.12) \\
& +\frac{n_{p}\left(c_{p o}-c_{d p} \mu_{p}\right)\left(c_{p f}+c_{p f f} \mu_{p}-c_{p o}\right)}{c_{p t}(1+\vartheta) \alpha_{w p} \mu_{p}}-\left(c_{p f}+c_{p f f} \mu_{p}-c_{p o}\right) \frac{\lambda_{p}}{\mu_{p}}
\end{aligned}
$$

The SOC is an unconstrained non-linear function of $n_{p} . n_{p}$ is optimized based on the necessary condition,

$\frac{\partial y_{s o c}}{\partial n_{p}}=\left(\frac{c_{p f}+c_{p f f} \mu_{p}-c_{p o}}{c_{p t}(1+\vartheta) \alpha_{w p}}\right)\left[\frac{c_{p o}}{\mu_{p}}-c_{d p}\right]-\frac{c_{c t} \alpha_{w c} \lambda_{c}^{2} l_{c} l_{p}}{\mu_{c}\left(l_{b}-n_{p} l_{p}\right)^{2}}=0$

From the equation above, the optimal $n_{p}$ is expressed below,

$$
\begin{aligned}
n_{p}^{*}\left(S 2 \mid \gamma_{c}=\gamma_{p}\right. & =1) \\
& =\frac{l_{b}}{l_{p}}-\lambda_{c} \sqrt{\left(\frac{l_{c}}{l_{p}}\right)\left(\frac{\mu_{p}}{\mu_{c}}\right) \frac{\left(c_{c t} \alpha_{w c}\right)\left(c_{p t}(1+\vartheta) \alpha_{w p}\right)}{\left(c_{p f}+c_{p f f} \mu_{p}-c_{p o}\right)\left(c_{p o}-c_{d p} \mu_{p}\right)}}
\end{aligned}
$$

The optimality condition is checked with the sufficient condition,

$\frac{\partial^{2} y_{s o c}}{\partial n_{p}^{2}}=-\frac{2 c_{c t} \alpha_{w c} \lambda_{c}^{2} l_{c} l_{p}^{2}}{\mu_{c}\left(l_{b}-n_{p} l_{p}\right)^{3}}<0$

The solution is a local maximum. 


\subsection{SOC model for Scenario 3: Some commercial vehicles double park}

Of the model for Scenario 3, its constraints are already explained in Section 5.5. Then, the next step is to take the objective function into account to find the optimal solutions.

\subsubsection{Objective function for Scenario 3}

Some commercial vehicles park on street. The commercial revenue demand $\left(\lambda_{c r}\right)$ equals the commercial breakeven demand $\left(\lambda_{c}^{*}\right)$. All passenger cars park on street. The car

revenue demand $\left(\lambda_{p r}\right)$ equals the arrival rate of passenger cars $\left(\lambda_{p}\right)$, as shown below,

$\lambda_{c r}=\lambda_{c}^{*}$

$\lambda_{p r}=\lambda_{p}$

Then, the social-optimum (SOC) function, $E q(7.1)$, becomes,

$$
\begin{gathered}
y_{s o c}(3)=\frac{c_{c o} \lambda_{c}^{*}}{\mu_{c}}+\frac{c_{p o} \lambda_{p}}{\mu_{p}}-c_{c t} \alpha_{w c}\left(\frac{\lambda_{c}^{*}}{n_{c} \mu_{c}}\right)^{\gamma_{c}} \lambda_{c}-c_{d c} \lambda_{c}^{*}-c_{d b}\left(\lambda_{c}-\lambda_{c}^{*}\right) \quad E q(7.14) \\
-\left[c_{p t}(1+\vartheta) \alpha_{w p}\left(\frac{\lambda_{p}}{n_{p} \mu_{p}}\right)^{\gamma_{p}}+c_{p t} f_{d b} t_{s}\left(\lambda_{c}-\lambda_{c}^{*}\right)\right] \lambda_{p}-c_{d p} \lambda_{p}
\end{gathered}
$$

The commercial breakeven demand $\left(\lambda_{c}^{*}\right)$ is estimated with $E q(5.22)$. Then, the SOC function becomes,

$$
\begin{array}{r}
y_{s o c}(3)=n_{c}\left[c_{c o}-c_{d c} \mu_{c}+c_{d b} \mu_{c}+c_{p t} f_{d b} t_{s} \lambda_{p} \mu_{c}\right]\left(\frac{c_{v} n_{e}-c_{c o} \tau_{e}}{c_{c t} \alpha_{w c} \mu_{c} \tau_{e}}\right)^{\frac{1}{\gamma_{c}}}-\left(\frac{c_{v} n_{e}}{\tau_{e}}-c_{c o}\right) \frac{\lambda_{c}}{\mu_{c}} \\
+\frac{c_{p o} \lambda_{p}}{\mu_{p}}-c_{p t}(1+\vartheta) \alpha_{w p}\left(\frac{\lambda_{p}}{n_{p} \mu_{p}}\right)^{\gamma_{p}} \lambda_{p}-c_{d p} \lambda_{p}-c_{d b} \lambda_{c}-c_{p t} f_{d b} t_{s} \lambda_{c} \lambda_{p}
\end{array}
$$




\subsubsection{Optimal value of $c_{c a}$}

The SOC is an unconstrained non-linear function of $c_{c o} . c_{c o}$ is optimized based on the necessary condition,

$$
\frac{\partial y_{s o c}}{\partial c_{c o}}=n_{c}\left(\frac{c_{v} n_{e}-c_{c o} \tau_{e}}{c_{c t} \alpha_{w c} \mu_{c} \tau_{e}}\right)^{\frac{1}{\gamma_{c}}}-\frac{n_{c}\left[c_{c o}-c_{d c} \mu_{c}+c_{d b} \mu_{c}+c_{p t} f_{d b} t_{s} \lambda_{p} \mu_{c}\right]}{\gamma_{c}\left(c_{c t} \alpha_{w c} \mu_{c}\right)^{\frac{1}{\gamma_{c}}}\left(\frac{c_{v} n_{e}}{\tau_{e}}-c_{c o}\right)^{\frac{\gamma_{c}-1}{\gamma_{c}}}}+\frac{\lambda_{c}}{\mu_{c}}=0
$$

From the equation above, the optimal $c_{c o}$ is expressed below,

$$
\frac{n_{c}\left[\gamma_{c} \frac{c_{v} n_{e}}{\tau_{e}}-\left(\gamma_{c}+1\right) c_{c o}^{*}(S 3)+c_{d c} \mu_{c}-c_{d b} \mu_{c}-c_{p t} f_{d b} \lambda_{p}\right]}{\gamma_{c}\left(c_{c t} \alpha_{w c} \mu_{c}\right)^{\frac{1}{\gamma_{c}}}\left(\frac{c_{v} n_{e}}{\tau_{e}}-c_{c o}^{*}(S 3)\right)^{\frac{\gamma_{c}-1}{\gamma_{c}}}}+\frac{\lambda_{c}}{\mu_{c}}=0
$$

If $\gamma_{c}=1$,

$c_{c o}^{*}\left(S 3 \mid \gamma_{c}=1\right)=\frac{c_{v} n_{e}}{2 \tau_{e}}+\frac{\left(c_{d c}-c_{d b}\right) \mu_{c}}{2}+\frac{c_{c t} \alpha_{w c} \lambda_{c}}{2 n_{c}}-\frac{c_{p t} f_{d b} \lambda_{p}}{2}$

The optimality condition is checked with the sufficient condition,

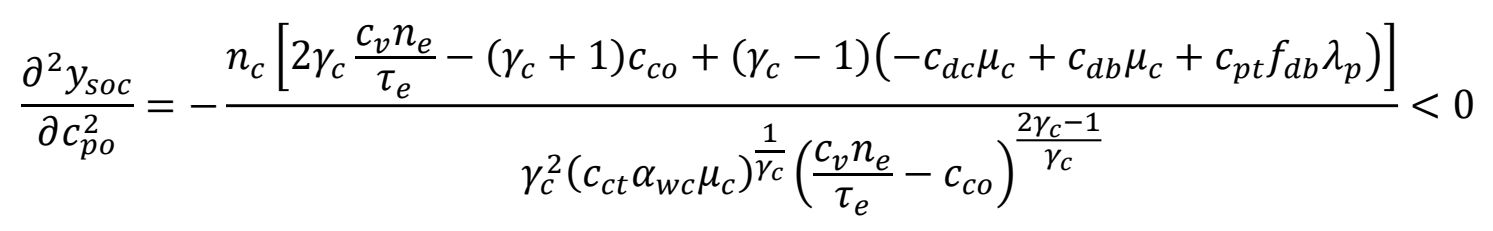

Therefore, the solution is a local maximum.

\subsubsection{Optimal value of $c_{p o}$}

Since the social optimum is a linear function of $c_{p o}$, the optimal $c_{p o}$ is one of extreme points of a feasible region. $E q(5.24)$ gives the feasible region. The optimal $c_{p o}$ is expressed below, 


$$
c_{p o}^{*}(S 3)=c_{p f}+c_{p f f} \mu_{p}-c_{p t}(1+\vartheta) \alpha_{w p} \mu_{p}\left(\frac{\lambda_{p}}{n_{p} \mu_{p}}\right)^{\gamma_{p}}-c_{p t} \mu_{p} f_{d b} t_{s}\left(\lambda_{c}-\lambda_{c}^{*}\right)
$$

\subsubsection{Optimal value of $n_{c}$ and $n_{p}$}

To reduce the model complication, both $\gamma_{c}$ and $\gamma_{p}$ are assumed to be 1.0 , the social optimum (SOC) is expressed below,

$$
\begin{aligned}
y_{s o c}\left(3 \mid \gamma_{c}=\gamma_{p}\right. & =1) \\
& =\frac{1}{c_{c t} \alpha_{w c}}\left(\frac{l_{b}-n_{p} l_{p}}{l_{c}}\right)\left(\frac{c_{c o}}{\mu_{c}}-c_{d c}+c_{d b}+c_{p t} f_{d b} t_{s} \lambda_{p}\right)\left(\frac{c_{v} n_{e}}{\tau_{e}}-c_{c o}\right) \\
& -\left(\frac{c_{v} n_{e}}{\tau_{e}}-c_{c o}\right) \frac{\lambda_{c}}{\mu_{c}}-c_{d b} \lambda_{c}-c_{p t} f_{d b} t_{s} \lambda_{p} \lambda_{c} \\
& +\left[\frac{c_{p o}}{\mu_{p}}-\frac{c_{p t}(1+\vartheta) \alpha_{w p} \lambda_{p}}{n_{p} \mu_{p}}-c_{d p}\right] \lambda_{p} \quad E q(7.19)
\end{aligned}
$$

The SOC is an unconstrained non-linear function of $n_{p} . n_{p}$ is optimized based on the necessary condition,

$$
\begin{gathered}
\frac{\partial y_{s o c}}{\partial n_{p}}=-\frac{1}{c_{c t} \alpha_{w c}}\left(\frac{l_{p}}{l_{c}}\right)\left(\frac{c_{c o}}{\mu_{c}}-c_{d c}+c_{d b}+c_{p t} f_{d b} t_{s} \lambda_{p}\right)\left(\frac{c_{v} n_{e}}{\tau_{e}}-c_{c o}\right) \\
+\frac{c_{p t}(1+\vartheta) \alpha_{w p} \lambda_{p}}{n_{p}^{2} \mu_{p}} \lambda_{p}=0
\end{gathered}
$$

From the equation above, the optimal $n_{p}$ is expressed below, 


$$
n_{p}^{*}\left(S 3 \mid \gamma_{c}=\gamma_{p}=1\right)=\lambda_{p} \sqrt{\frac{\left(c_{c t} \alpha_{w c}\right)\left[c_{p t}(1+\vartheta) \alpha_{w p}\right]}{\left(\frac{c_{v} n_{e}}{\tau_{e}}-c_{c o}\right)\left(c_{c o}-c_{d c} \mu_{c}+c_{d b} \mu_{c}+c_{p t} f_{d b} \lambda_{p}\right)}\left(\frac{\mu_{c}}{\mu_{p}}\right)\left(\frac{l_{c}}{l_{p}}\right)}
$$

The optimality condition is checked with the sufficient condition,

$$
\frac{\partial^{2} y_{s o c}}{\partial n_{p}^{2}}=-\frac{2 c_{p t}(1+\vartheta) \alpha_{w p} \lambda_{p}^{2}}{n_{p}^{3} \mu_{p}}<0
$$

The solution is a local maximum.

\subsection{SOC model for Scenario 4: All parking options are selected}

Of the model for Scenario 4, its constraints are already explained in Section 5.6. Then, the next step is to take the objective function into account to find the optimal solutions.

\subsubsection{Objective function for Scenario 4}

Some commercial vehicles park on street. The commercial revenue demand $\left(\lambda_{c r}\right)$ equals the commercial breakeven demand $\left(\lambda_{c}^{*}\right)$. Some passenger cars park on street. The car revenue demand $\left(\lambda_{p r}\right)$ equals the car breakeven demand $\left(\lambda_{p}^{*}\right)$, as shown below,

$$
\begin{aligned}
& \lambda_{c r}=\lambda_{c}^{*} \\
& \lambda_{p r}=\lambda_{p}^{*}
\end{aligned}
$$

Then, the social-optimum function, $E q(7.1)$, becomes, 


$$
\begin{gathered}
y_{s o c}(4)=\frac{c_{c o} \lambda_{c}^{*}}{\mu_{c}}+\frac{c_{p o} \lambda_{p}^{*}}{\mu_{p}}-c_{c t} \alpha_{w c}\left(\frac{\lambda_{c}^{*}}{n_{c} \mu_{c}}\right)^{\gamma_{c}} \lambda_{c}-c_{d c} \lambda_{c}^{*}-c_{d b}\left(\lambda_{c}-\lambda_{c}^{*}\right) \quad E q(7.21) \\
-\left[c_{p t}(1+\vartheta) \alpha_{w p}\left(\frac{\lambda_{p}^{*}}{n_{p} \mu_{p}}\right)^{\gamma_{p}}+c_{p t} f_{d b} t_{s}\left(\lambda_{c}-\lambda_{c}^{*}\right)\right] \lambda_{p}-c_{d p} \lambda_{p}^{*}
\end{gathered}
$$

The commercial breakeven demand $\left(\lambda_{c}^{*}\right)$ is estimated with $E q(5.30)$ and the car breakeven demand $\left(\lambda_{p}^{*}\right)$ is estimated with $E q(5.32)$.

Let $A=\left(c_{c t} \alpha_{w c} \mu_{c}\right)^{\frac{1}{\gamma_{c}}}$

Let $B=\left(c_{p t}(1+\vartheta) \alpha_{w p} \mu_{p}\right)^{\frac{1}{\gamma_{p}}}$

Let $D=c_{p f}+c_{p f f} \mu_{p}-c_{p o}-c_{p t} f_{d b} t_{s} \mu_{p}\left\{\lambda_{c}-n_{c} \mu_{c}\left(\frac{c_{v} n_{e}-c_{c o} \tau_{e}}{c_{c t} \alpha_{w c} \mu_{c} \tau_{e}}\right)^{\frac{1}{\gamma_{c}}}\right\}$

The social-optimum function becomes,

$$
\begin{gathered}
y_{s o c}(4)=\frac{n_{c}\left(c_{c o}-c_{d c} \mu_{c}+c_{d b} \mu_{c}\right)}{A}\left(\frac{c_{v} n_{e}}{\tau_{e}}-c_{c o}\right)^{\frac{1}{\gamma_{c}}}-\left(\frac{c_{v} n_{e}}{\tau_{e}}-c_{c o}\right) \frac{\lambda_{c}}{\mu_{c}} E q(7.22) \\
-\left(c_{p f}+c_{p f f} \mu_{p}-c_{p o}\right) \frac{\lambda_{p}}{\mu_{p}}-c_{d b} \lambda_{c}+\frac{n_{p}\left(c_{p o}-c_{d p} \mu_{p}\right)}{B} D^{\frac{1}{\gamma_{p}}}
\end{gathered}
$$

\subsubsection{Optimal value of $c_{c a}$}

The social optimum is an unconstrained non-linear function of $c_{c o} \cdot c_{c o}$ is optimized based on the necessary condition,

$\frac{\partial y_{s o c}}{\partial c_{c o}}=0$ 


$$
\begin{gathered}
\frac{n_{c}}{A}\left(\frac{c_{v} n_{e}}{\tau_{e}}-c_{c o}^{*}(S 4)\right)^{\frac{1}{\gamma_{c}}}-\frac{n_{c}\left(c_{c o}^{*}(S 4)-c_{d c} \mu_{c}+c_{d b} \mu_{c}\right)}{\gamma_{c} A\left(\frac{c_{v} n_{e}}{\tau_{e}}-c_{c o}^{*}(S 4)\right)^{\frac{\gamma_{c}-1}{\gamma_{c}}}} \\
-\frac{c_{p t} f_{d b} t_{s} n_{c} \mu_{c} n_{p} \mu_{p}\left(c_{p o}-c_{d p} \mu_{p}\right)}{\gamma_{c} \gamma_{p} A B D^{\frac{\gamma_{p}-1}{\gamma_{p}}}\left(\frac{c_{v} n_{e}}{\tau_{e}}-c_{c o}^{*}(S 4)\right)^{\frac{\gamma_{c}}{\gamma_{c}}}+\frac{\lambda_{c}}{\mu_{c}}=0 \quad E q(7.23)} \\
\text { If } \gamma_{c}=\gamma_{p}=1, \quad \begin{array}{c}
c_{v} n_{e} \\
2 \tau_{e}
\end{array} \frac{\left(c_{d c}-c_{d b}\right) \mu_{c}}{2}+\frac{\lambda_{c} c_{c t} \alpha_{w c}}{2 n_{c}}-\frac{f_{d b} t_{s} \mu_{c} n_{p}\left(c_{p o}-c_{d p} \mu_{p}\right)}{2(1+\vartheta) \alpha_{w p}} \\
c_{c o}^{*}\left(S 4 \mid \gamma_{c}=\gamma_{p}=1\right)=1
\end{gathered}
$$

The optimality condition is checked with the sufficient condition,

$$
\frac{\partial^{2} y_{s o c}\left(\gamma_{c}=\gamma_{p}=1\right)}{\partial c_{c o}^{2}}=-\frac{2}{c_{c t} \alpha_{w c} \mu_{c}}\left(\frac{l_{b}-n_{p} l_{p}}{l_{c}}\right)<0
$$

Therefore, the solution is a local maximum.

\subsubsection{Optimal value of $c_{p o}$}

The social optimum is an unconstrained non-linear function of $c_{p o} \cdot c_{p o}$ is optimized based on the necessary condition,

$$
\frac{\partial y_{s o c}}{\partial c_{p o}}=\frac{n_{p}}{B} D^{\frac{1}{\gamma_{p}}}-\frac{n_{p}\left(c_{p o}-c_{d p} \mu_{p}\right)}{\gamma_{p} B}\left(D^{\frac{1-\gamma_{p}}{\gamma_{p}}}\right)+\frac{\lambda_{p}}{\mu_{p}}=0
$$

From the equation above, the optimal $c_{p o}$ is expressed below,

$$
\frac{n_{p}}{B} D^{\frac{1}{\gamma_{p}}}-\frac{n_{p}\left(c_{p o}-c_{d p} \mu_{p}\right)}{\gamma_{p} B}\left(D^{\frac{1-\gamma_{p}}{\gamma_{p}}}\right)+\frac{\lambda_{p}}{\mu_{p}}=0
$$




$$
\begin{aligned}
& \text { If } \gamma_{c}=\gamma_{p}=1 \text {, } \\
& \begin{aligned}
c_{p o}^{*}\left(S 4 \mid \gamma_{c}=\gamma_{p}=1\right) \\
\quad=\frac{\left(c_{p f}+c_{p f f} \mu_{p}\right)}{2}+\frac{c_{d p} \mu_{p}}{2}+\frac{\lambda_{p} c_{p t}(1+\vartheta) \alpha_{w p}}{2 n_{p}}-\frac{c_{p t} \mu_{p} f_{d b} t_{s}}{2}\left[\lambda_{c}-\lambda_{c}^{*}\right]
\end{aligned}
\end{aligned}
$$

The optimality condition is checked with the sufficient condition,

$$
\frac{\partial^{2} y_{s o c}\left(\gamma_{c}=\gamma_{p}=1\right)}{\partial c_{p o}^{2}}=-\frac{2 n_{p}}{c_{p t}(1+\vartheta) \alpha_{w p} \mu_{p}}<0
$$

The solution is a local maximum.

\subsubsection{Optimal value of $n_{c}$ and $n_{p}$}

To reduce the model complication, both $\gamma_{c}$ and $\gamma_{p}$ are assumed to be 1.0, the social optimum (SOC) is expressed below,

$$
\begin{aligned}
y_{s o c}\left(S 4 \mid \gamma_{c}\right. & \left.=\gamma_{p}=1\right) \frac{\left(c_{c o}-c_{d c} \mu_{c}+c_{d b} \mu_{c}\right)}{A}\left(\frac{l_{b}-n_{p} l_{p}}{l_{c}}\right)\left(\frac{c_{v} n_{e}}{\tau_{e}}-c_{c o}\right) E q(7.27) \\
& -\left(\frac{c_{v} n_{e}}{\tau_{e}}-c_{c o}\right) \frac{\lambda_{c}}{\mu_{c}}-\left(c_{p f}+c_{p f f} \mu_{p}-c_{p o}\right) \frac{\lambda_{p}}{\mu_{p}}-c_{d b} \lambda_{c}+\frac{n_{p}\left(c_{p o}-c_{d p} \mu_{p}\right) D}{B}
\end{aligned}
$$

The SOC is an unconstrained non-linear function of $n_{p} . n_{p}$ is optimized based on the necessary condition,

$$
\begin{aligned}
\frac{\partial y_{s o c}}{\partial n_{p}}=\left(\frac{-l_{p}}{l_{c}}\right) & \frac{\left(c_{c o}-c_{d c} \mu_{c}+c_{d b} \mu_{c}\right)}{A}\left(\frac{c_{v} n_{e}}{\tau_{e}}-c_{c o}\right) \quad E q(7.28) \\
+ & \frac{\left(c_{p o}-c_{d p} \mu_{p}\right)}{B}\left[D-n_{p} c_{p t} f_{d b} t_{s} \mu_{p} \mu_{c}\left(\frac{c_{v} n_{e}-c_{c o} \tau_{e}}{c_{c t} \alpha_{w c} \mu_{c} \tau_{e}}\right)\left(\frac{l_{p}}{l_{c}}\right)\right]=0
\end{aligned}
$$


From the equation above, the optimal $n_{p}$ is expressed below,

$$
\begin{aligned}
& n_{p}^{*}\left(S 4 \mid \gamma_{c}=\gamma_{p}=1\right)=\frac{l_{b}}{2 l_{p}}-\frac{\lambda_{c} \alpha_{w c}}{2}\left(\frac{l_{c}}{l_{p}}\right)\left(\frac{c_{c t}}{\frac{c_{v} n_{e}}{\tau_{e}}-c_{c o}}\right) \quad E q(7.29) \\
& +\frac{\alpha_{w c}}{2 f_{d b} t_{s} \mu_{p}}\left(\frac{l_{c}}{l_{p}}\right)\left(\frac{c_{c t}}{c_{p t}}\right)\left(\frac{c_{p f}+c_{p f f} \mu_{p}-c_{p o}}{\frac{c_{v} n_{e}}{\tau_{e}}-c_{c o}}\right)-\frac{(1+\vartheta) \alpha_{w p}}{2 f_{d b} t_{s} \mu_{c}}\left(\frac{c_{c o}-c_{d c} \mu_{c}+c_{d b} \mu_{c}}{c_{p o}-c_{d p} \mu_{p}}\right)
\end{aligned}
$$

The optimality condition is checked with the sufficient condition,

$$
\frac{\partial^{2} y_{s o c}\left(\gamma_{c}=\gamma_{p}=1\right)}{\partial n_{p}^{2}}=-\left(\frac{2 l_{p}}{l_{c}}\right)\left(c_{p o}-c_{d p} \mu_{p}\right)\left(\frac{c_{v} n_{e}}{\tau_{e}}-c_{c o}\right) \frac{f_{d b} t_{s}}{(1+\vartheta) \alpha_{w p} c_{c t} \alpha_{w c}}<0
$$

The solution is a local maximum.

\subsection{Solution summary and interpretation}

This section analyzes how the decision variable is affected by parameters and other variables. Before interpreting the solutions, all solutions are summarized in Table 7.1. 
Table 7.1 Solutions to social-optimum models for 4 scenarios

\begin{tabular}{|c|c|}
\hline Solutions & Equation \\
\hline $\begin{array}{l}\text { Scenario1 All vehicles park on street } \\
c_{c o}^{*}(S 1)=\frac{c_{v} n_{e}}{\tau_{e}}-c_{c t} \alpha_{w c} \mu_{c}\left(\frac{\lambda_{c}}{n_{c} \mu_{c}}\right)^{\gamma_{c}} \\
c_{p o}^{*}(S 1)=c_{p f}+c_{p f f} \mu_{p}-c_{p t}(1+\vartheta) \alpha_{w p} \mu_{p}\left(\frac{\lambda_{p}}{n_{p} \mu_{p}}\right)^{\gamma_{p}} \\
n_{p}^{*}\left(S 1 \mid \gamma_{c}=\gamma_{p}=1\right)=\frac{l_{b}}{l_{p}+\left(\frac{\lambda_{c}}{\lambda_{p}}\right) \sqrt{l_{c} l_{p} \frac{c_{c t} \alpha_{w c}}{c_{p t}(1+\vartheta) \alpha_{w p}}\left(\frac{\mu_{p}}{\mu_{c}}\right)}}\end{array}$ & 7.4 \\
\hline $\begin{array}{l}\text { Scenario } 2 \text { Some passenger cars park off street } \\
\begin{array}{c}c_{c o}^{*}(S 2)=\frac{c_{v} n_{e}}{\tau_{e}}-c_{c t} \alpha_{w c} \mu_{c}\left(\frac{\lambda_{c}}{n_{c} \mu_{c}}\right)^{\gamma_{c}} \\
\frac{n_{p}\left[\gamma_{p}\left(c_{p f}+c_{p f f} \mu_{p}\right)-c_{p o}^{*}(\boldsymbol{S} 2)\left(\gamma_{p}+1\right)+c_{d p} \mu_{p}\right]}{\left[c_{p t}(1+\vartheta) \alpha_{w p} \mu_{p}\right]^{\frac{1}{\gamma_{p}}} \gamma_{p}\left(c_{p f}+c_{p f f} \mu_{p}-c_{p o}^{*}(\boldsymbol{S} 2)\right)^{\frac{\gamma_{p}-1}{\gamma_{p}}}}+\frac{\lambda_{p}}{\mu_{p}}=0 \\
c_{p o}^{*}\left(S 2 \mid \gamma_{p}=1\right)=\frac{c_{p f}+c_{p f f} \mu_{p}}{2}+\frac{c_{d p} \mu_{p}}{2}+\frac{c_{p t}(1+\vartheta) \alpha_{w p}}{2}\left(\frac{\lambda_{p}}{n_{p}}\right) \\
n_{p}^{*}\left(S 2 \mid \gamma_{c}=\gamma_{p}=1\right) \\
l_{p}-\lambda_{c} \sqrt{\left(\frac{l_{c}}{l_{p}}\right)\left(\frac{\mu_{p}}{\mu_{c}}\right) \frac{\left(c_{c t} \alpha_{w c}\right)\left(c_{p t}(1+\vartheta) \alpha_{w p}\right)}{\left(c_{p f}+c_{p f f} \mu_{p}-c_{p o}\right)\left(c_{p o}-c_{d p} \mu_{p}\right)}}\end{array}\end{array}$ & 7.11 \\
\hline
\end{tabular}


Table 7.1 Solutions to social-optimum models for 4 scenarios (continued)

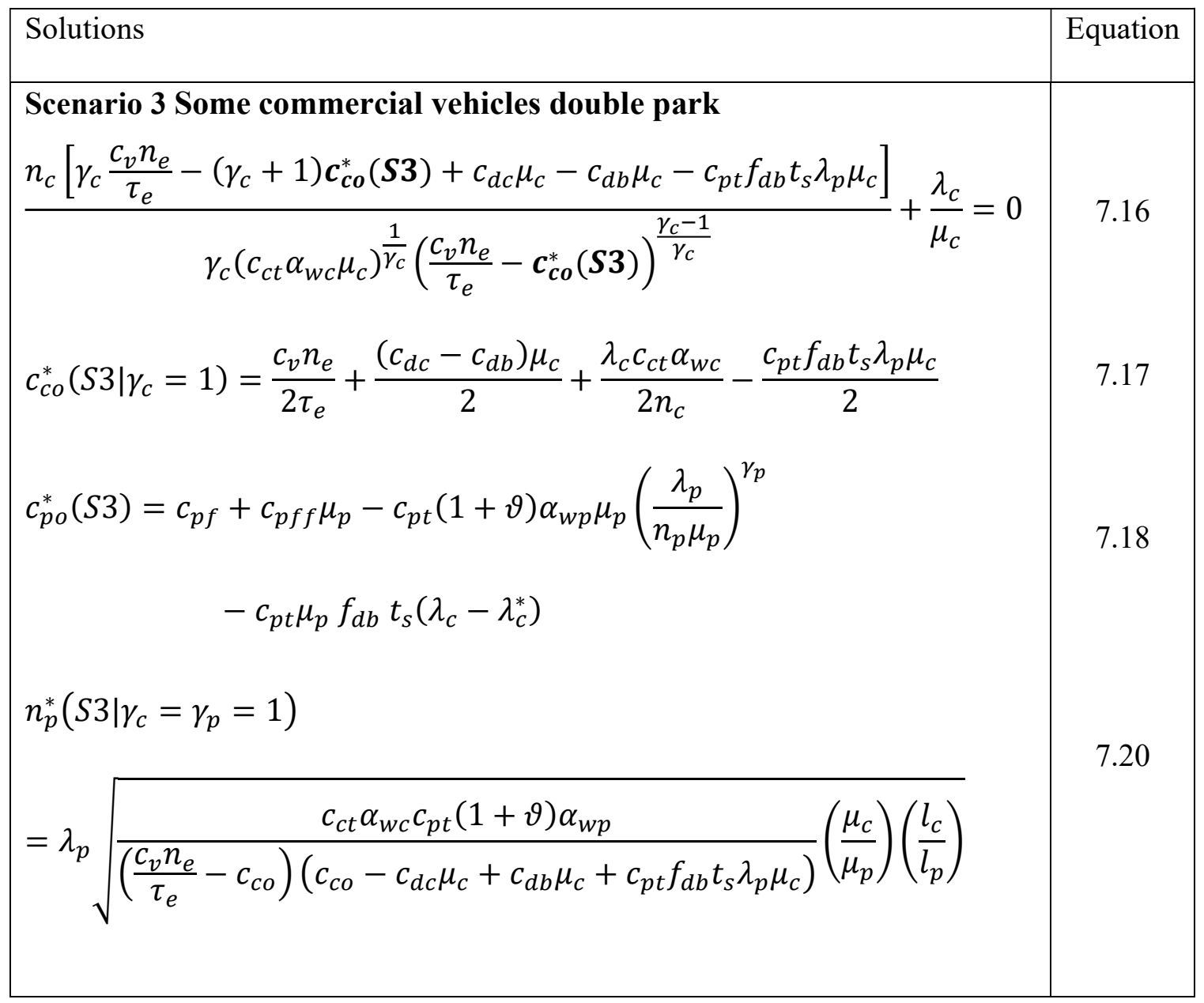


Table 7.1 Solutions to social-optimum models for 4 scenarios (continued)

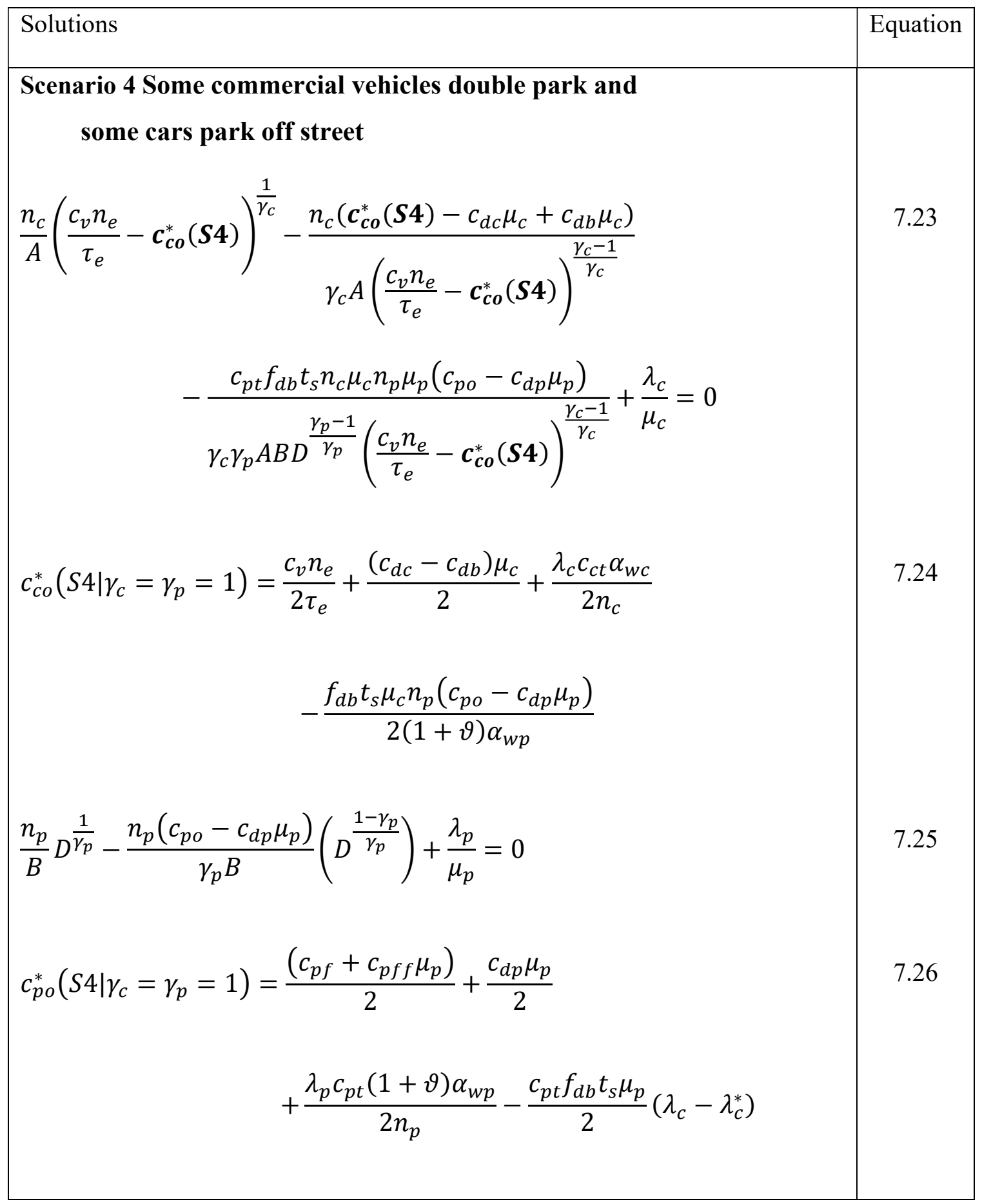


Table 7.1. Solutions to social-optimum models for 4 scenarios (continued)

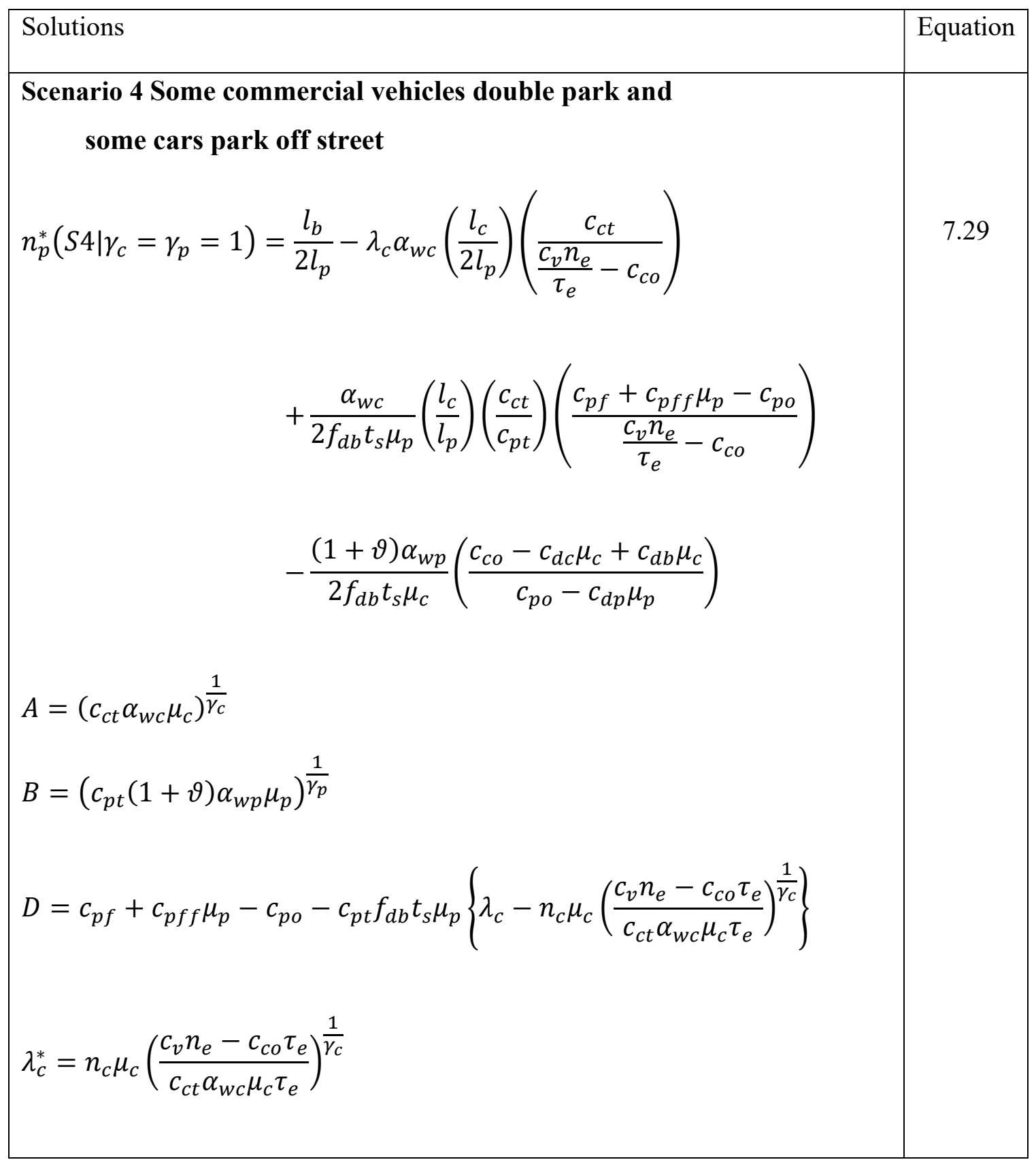

From Table 7.1, the interpretation to variable parameters and decision variables is summarized in Tables 7.2 to 7.4 . 
Table 7.2 Interpretation of optimal loading zone meter rate for SOC models

\begin{tabular}{|c|l|}
\hline Variable & \multicolumn{1}{|c|}{ Interpretation of optimal $c_{c o}$} \\
\hline$\frac{c_{v} n_{e}}{\tau_{e}} \propto c_{c o}^{*}$ & If the cost of the other parking option is high, the meter rate can be high. \\
\hline$n_{c} \propto \frac{1}{c_{c o}^{*}}$ & $\begin{array}{l}\frac{\text { Scenarios } 1 \text { and } 2}{\text { If parking supply is high, the parking search cost will be low. The other }} \\
\text { component of on-street parking, the meter rate, can be high. }\end{array}$ \\
\hline$\lambda_{c} \propto \frac{1}{c_{c o}^{*}}$ & $\begin{array}{l}\frac{\text { Scenarios } 1 \text { and } 2}{\text { If the arrival rate of commercial vehicles is high, the meter rate will be low }} \\
\text { in order to keep all commercial vehicles park on street. }\end{array}$ \\
\hline$\lambda_{c} \propto c_{c o}^{*}$ & $\begin{array}{l}\text { If the arrival rate of commercial vehicles is high, the meter rate will be } \\
\text { high in order to have a few vehicles park on street. The purpose is to keep } \\
\text { the cost of two options equal. }\end{array}$ \\
\hline$c_{d b} \propto \frac{\text { Scenarios } 3 \text { and } 4}{c_{c o}^{*}}$ & $\begin{array}{l}\text { Scenario 3 and 4 } \\
\text { If delays due to double parking are high, the meter rate will be low in order } \\
\text { to decrease the number of double-parking events. }\end{array}$ \\
\hline
\end{tabular}


Table 7.3 Interpretation of optimal parking space meter rate for SOC models

\begin{tabular}{|c|c|}
\hline Parameter & Interpretation of optimal $c_{p o}$ \\
\hline$c_{p f} \propto c_{p o}^{*}$ & If the cost of the other parking option is high, the meter rate can be high. \\
\hline$n_{p} \propto \frac{1}{c_{p o}^{*}}$ & $\begin{array}{l}\text { Scenarios } 1 \text { and } 3 \\
\text { If parking supply is high, the parking search cost will be low. The other } \\
\text { component of on-street parking, the meter rate, can be high. }\end{array}$ \\
\hline$\lambda_{c} \propto \frac{1}{c_{p o}^{*}}$ & $\begin{array}{l}\text { Scenarios } 3 \text { and } 4 \\
\text { If arrival rate of commercial vehicles is high, the number of double- } \\
\text { parking events will be high. That increases travel delays to cars parking } \\
\text { on street. The meter rate has to decrease in order to prevent an increase in } \\
\text { the cost of on-street parking. }\end{array}$ \\
\hline$\lambda_{p} \propto \frac{1}{c_{p o}^{*}}$ & $\begin{array}{l}\text { Scenarios } 1 \text { and } 2 \\
\text { If the arrival rate of passenger cars is high, the meter rate will be low in } \\
\text { order to keep all commercial vehicles park on street. }\end{array}$ \\
\hline$\lambda_{p} \propto c_{p o}^{*}$ & $\begin{array}{l}\text { Scenarios } 3 \text { and } 4 \\
\text { If the arrival rate of passenger cars is high, the meter rate will be high in } \\
\text { order to have a few vehicles park on street. The purpose is to keep the cost } \\
\text { of two options equal. }\end{array}$ \\
\hline
\end{tabular}

Table 7.4 Interpretation of optimal curb length allocation for SOC models

\begin{tabular}{|c|l|}
\hline Parameter & \multicolumn{1}{c|}{ Interpretation of optimal $n_{p}$} \\
\hline$l_{b} \propto n_{p}^{*}$ & $\begin{array}{l}\text { The longer the block length, the more the block is allocated to parking } \\
\text { spaces. }\end{array}$ \\
\hline$\lambda_{p} \propto n_{p}^{*}$ & $\begin{array}{l}\underline{\text { Scenario 1 and } 3} \\
\text { The higher the arrival rate of parking cars, the more the number of } \\
\text { parking spaces to prevent an increase in the parking search cost. }\end{array}$ \\
\hline$\lambda_{c} \propto \frac{1}{n_{p}^{*}}$ & $\begin{array}{l}\underline{\text { Scenarios 2 and 4 }} \\
\text { If the arrival rate of commercial vehicles is high, the number of loading } \\
\text { zones will be high. This leads to low number of parking spaces. }\end{array}$ \\
\hline
\end{tabular}




\subsection{Steps to find optimal solutions}

From the optimal solutions (Section 7.7), for most scenarios, one decision variable is a function of the other two decision variables. It is practical to vary one of the variables and calculate the other variables. $c_{c o}^{*}$ and $c_{p o}^{*}$ are similar because both are meter rates while $n_{c}^{*}$ is different from the others since it is related to supply allocation. Therefore, it is to vary $n_{c}$ and calculate the others. The steps to find optimal solutions are described below,

6) Start with $n_{c}$ equal to 1

7) Calculate $c_{c o}^{*}$ and $c_{p o}^{*}$ using equations shown below,

\begin{tabular}{|c|c|c|}
\hline \multirow{2}{*}{ Scenario } & \multicolumn{2}{|c|}{ Equation for calculating the optimal meter rates } \\
\cline { 2 - 3 } & $c_{c o}^{*}$ & $c_{p o}^{*}$ \\
\hline 1 & $\mathrm{Eq} \mathrm{7.3}$ & $\mathrm{Eq} \mathrm{7.4}$ \\
\hline 2 & $\mathrm{Eq} 7.9$ & $\mathrm{Eq} 7.10$ \\
\hline 3 & $\mathrm{Eq} \mathrm{7.16}$ & $\mathrm{Eq} 7.18$ \\
\hline 4 & $\mathrm{Eq} 7.23$ & $\mathrm{Eq} 7.25$ \\
\hline
\end{tabular}

8) Calculate the revenue

9) Check if $n_{c}$ equals $l_{b} / l_{c}$. If not, increase $n_{c}$ and repeat steps 2,3 , and 4

10) Find $n_{c}$ whose social optimum is maximum

\subsection{Conclusion}

This chapter proposes a social optimum maximization model for on-street parking system. The decision variables are meter rate of loading zones $\left(c_{c o}\right)$, meter rate of parking

spaces $\left(c_{p o}\right)$, and the number of parking spaces $\left(n_{p}\right)$. The first two variables are to set fees for parking on street. The last variable is to allocate curb length to loading zones and parking spaces.

One decision variable is optimized at a time while the other variables keep constant. Optimal solutions are different between scenarios. However, the impacts of parameters and 
other variables on a decision variable in different scenarios are similar: the same direction but different magnitude. In the next chapter, a case study is conducted to demonstrate the optimal solutions and the impacts of parameters and other decision variables. 


\section{Chapter 8 Case Study for Social Optimum Models}

\subsection{Introduction}

This chapter demonstrates how the social optimum (SOC) models optimize meter rates and curb length allocation under given circumstances. Since there are 4 scenarios for developing parking models (Section 4.6), 4 sets of data are assumed for case studies; one set for one scenario. To facilitate comparisons, values of most variables are kept the same, including value of time, unit costs, length and service rate of loading zones and parking spaces, and coefficient for estimating parking search time, as shown in Table 6.1. However, demands must be changed.

The difference among the sets of data is arrival rates of parking commercial vehicles and parking passenger cars. Of the first set, the arrival rates of both vehicle types are low. All the vehicles park on street. Of the second set, the arrival rate of commercial vehicles is low but the arrival rate of passenger cars is high. All the commercial vehicles park on street. Some passenger cars park on street and others park off street. Of the third set, the arrival rate of commercial vehicles is high but the arrival rate of passenger cars is low. Some commercial vehicles park on street but others double park. Of the fourth set, the arrival rates of commercial vehicles and passenger cars are high. All parking options (onstreet parking, double-parking, and off-street parking) are chosen. The base data for the four cases is shown in Table 6.2. 


\subsection{Optimal solutions for passenger car parking equivalent}

As explained in Section 6.2, there are no impacts of vehicle types. In addition, the equivalent curb length allocation is constant. The impact of arrival rates on the meter rate can be analyzed clearly. From Chapter 4, the parking options for passenger cars are parking on street and parking off street. The two cases are analyzed: parking on street only and parking on- and off-street.

\subsubsection{Parking on street only}

As $\lambda_{a}$ increases, the parking search cost increases. In order to keep the on-street option cheaper than the off-street option, the meter rate has to decrease. The results are shown in Table 8.1. If $\lambda_{a}$ is very high, the meter rate will be negative. This means that drivers get paid in order to park on street. This is not common in many urban areas.

Table 8.1 Solutions of SOC models: vehicle equivalent parking on street

\begin{tabular}{|r|r|r|r|r|}
\hline \multicolumn{1}{|c|}{$\lambda_{p}$} & \multicolumn{1}{c|}{$c_{p o}^{*}$} & \multicolumn{1}{c|}{$\lambda_{p r}$} & Revenue & Social Optimum \\
\hline 7.00 & 4.17 & 7.00 & 14.60 & 10.78 \\
\hline 7.49 & 4.05 & 7.49 & 15.17 & 10.64 \\
\hline 12.25 & 2.46 & 12.25 & 15.07 & -2.08 \\
\hline 17.50 & -0.18 & 17.50 & -1.61 & -49.24 \\
\hline 22.25 & -3.38 & 22.25 & -37.60 & -133.72 \\
\hline
\end{tabular}

\subsubsection{Parking on street and off street}

As $\lambda_{a}$ increases, the meter rate increases but the revenue demand decreases. This way reduces the parking search cost which can maximize the social optimum, as shown in Table 8.2. 
Table 8.2 Solutions of SOC models: vehicle equivalent parking on- and off-street

\begin{tabular}{|r|r|r|r|r|}
\hline$\lambda_{p}$ & \multicolumn{1}{|c|}{$c_{p o}^{*}$} & \multicolumn{1}{c|}{$\lambda_{p r}$} & \multicolumn{1}{l|}{ Revenue } & Social optimum \\
\hline 7.49 & 4.05 & 7.49 & 15.17 & 10.64 \\
\hline 12.25 & 4.31 & 6.38 & 13.74 & 8.69 \\
\hline 17.50 & 4.50 & 5.43 & 12.21 & 7.13 \\
\hline 22.25 & 4.62 & 4.73 & 10.93 & 6.09 \\
\hline
\end{tabular}

Considering Table 8.1 and Table 8.2 , it is practical to decrease the meter rate as the arrival rate increases while all the vehicles park on street, according to Table 8.1. When the meter rate equals the meter rate in Table 8.2 , it is practical to keep the meter rate the same and some vehicles park off street. It is not reasonable to keep all vehicles park on street by lowering the meter rate because the meter rate can be negative. It can be inferred that drivers are paid to park on street, according to Table 8.1 .

\subsection{Optimal solutions for parking scenarios}

Each scenario is optimized at a time. Steps to maximize the social optimum (SOC) is already explained in Section 7.8. The optimal curb length allocation and its optimal meter rates lead to the highest SOC. The numerical results include the optimal meter rates of both parking types, the revenue demand of both vehicle types, and the social optimum (SOC).

If the revenue demand is not a function of the number of parking spaces $\left(n_{p}\right)$, it will not be shown in plots.

\subsubsection{Optimal solution for scenario-1 social optimum model}

The management strategy of scenario 1 is to keep all vehicles park on street. From $E q(7.3)$, if the curb allocation is low, the optimal meter rates of loading zones and parking spaces will be low, as shown in Figure 8.1. 


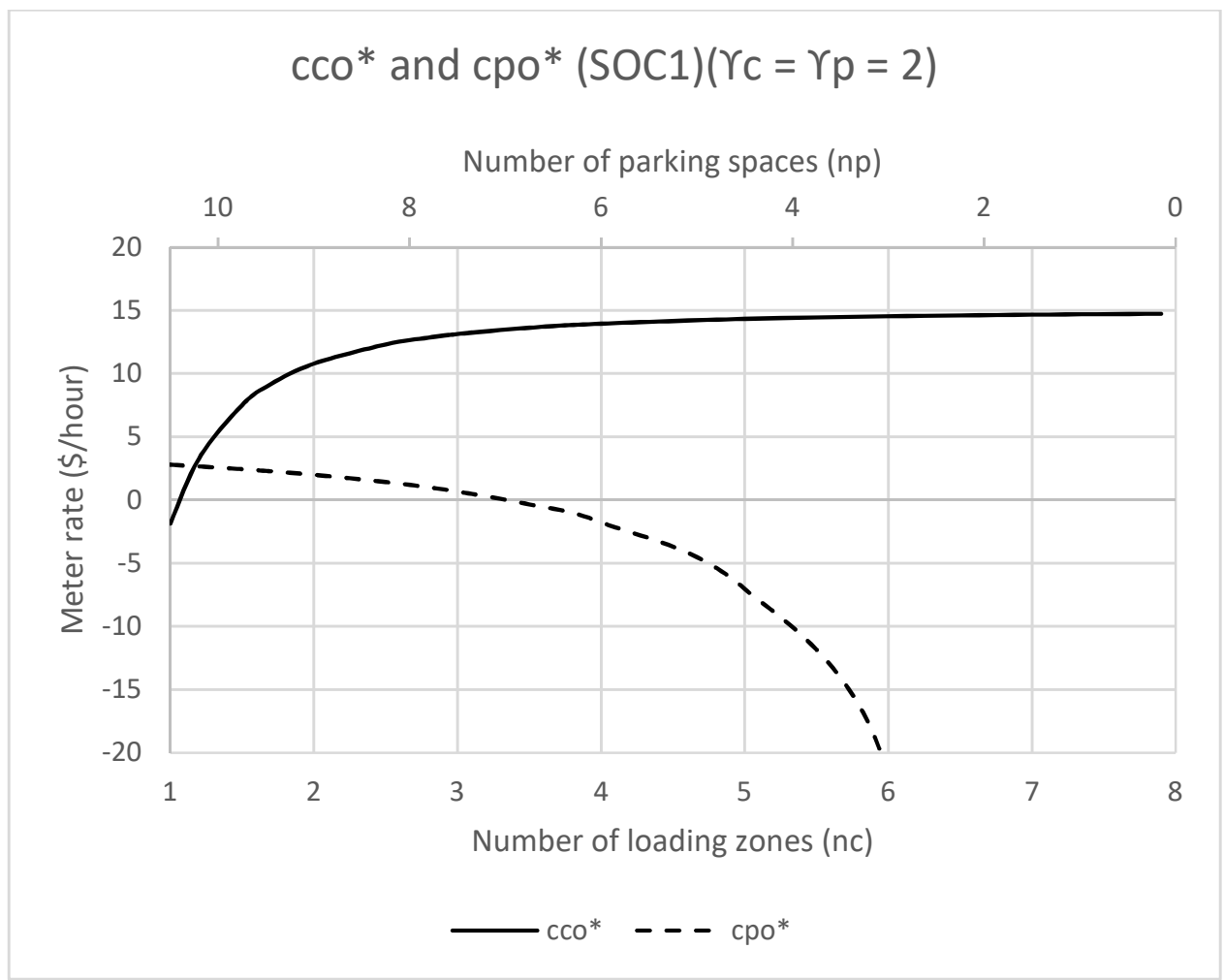

Figure 8.1 Optimal meter rates of scenario-1 social optimum model

For low curb allocation, the meter rate of parking spaces is lower than the meter rate of loading zones. To prevent a low value of social optimum, the majority of curb length is allocated to parking spaces, as shown in Figure 8.2. 


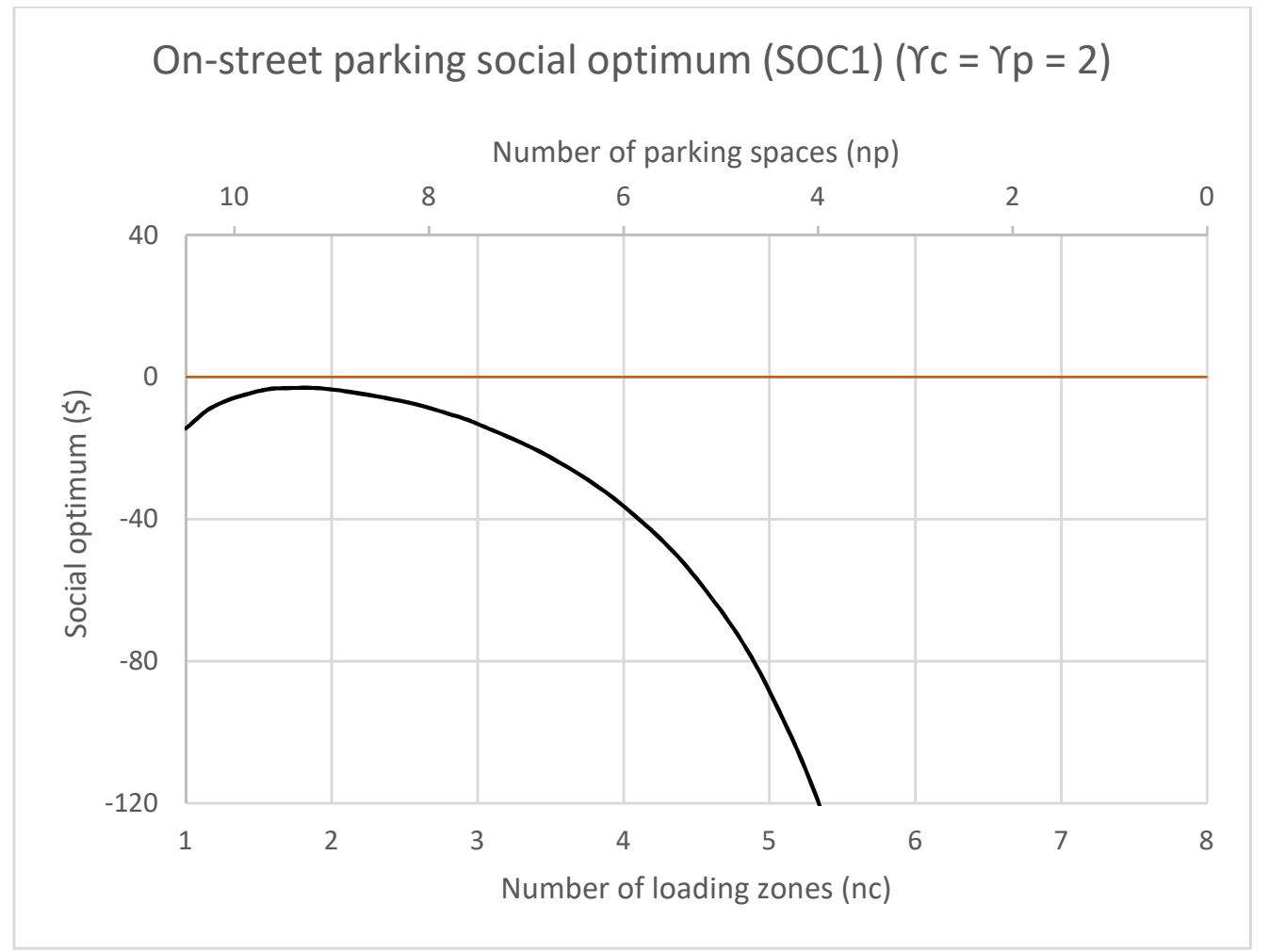

Figure 8.2 On-street scenario-1 social optimum

\subsubsection{Optimal solution for scenario-2 social optimum model}

The management strategy of scenario-2 is to keep all commercial vehicles park on street and to let some passenger cars park off street. For loading zones, the commercial revenue demand $\left(\lambda_{c r}\right)$ is constant. From $E q(7.9)$, if its curb allocation $\left(n_{c}\right)$ increases, the optimal meter rate of loading zones $\left(c_{c o}^{*}\right)$ will increase. The social optimum (SOC) from loading zones will increase.

For parking spaces, from $E q(7.11)$, if its curb allocation $\left(n_{p}\right)$ decreases, the optimal meter rate of parking spaces $\left(c_{p o}^{*}\right)$ will increase. However, the car revenue demand $\left(\lambda_{p r}\right)$ will decrease. The SOC from parking spaces will decrease. 
Based on data from Table 6.1 and 6.2, if the number of loading zones $\left(n_{c}\right)$ increases, $c_{c o}^{*}$ will increase but $c_{p o}^{*}$ will slightly increase, as shown in Figure 8.3. $\lambda_{c r}$ will be constant but $\lambda_{p r}$ will decrease, as shown in Figure 8.4.

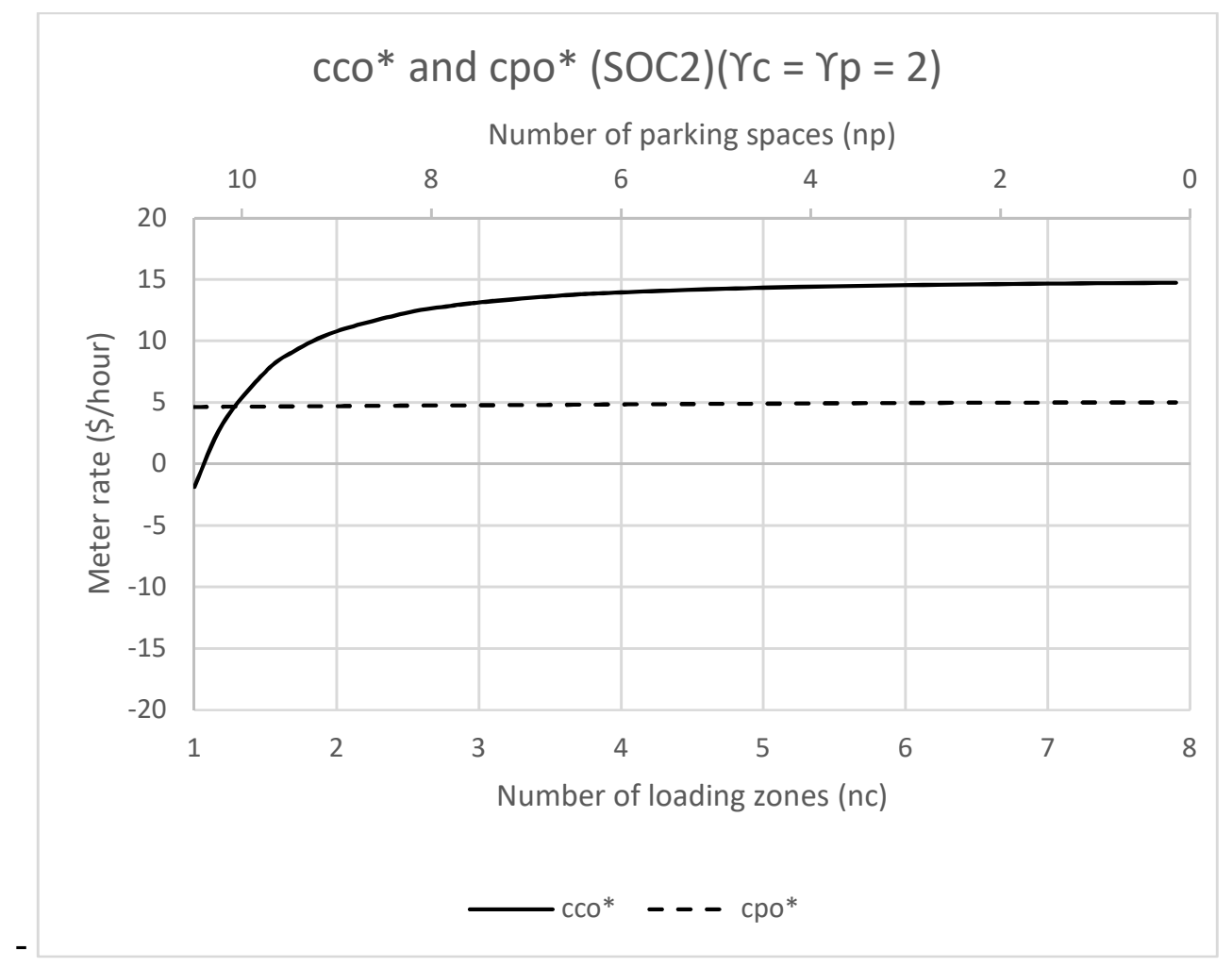

Figure 8.3 Optimal meter rates of scenario-2 social optimum model 


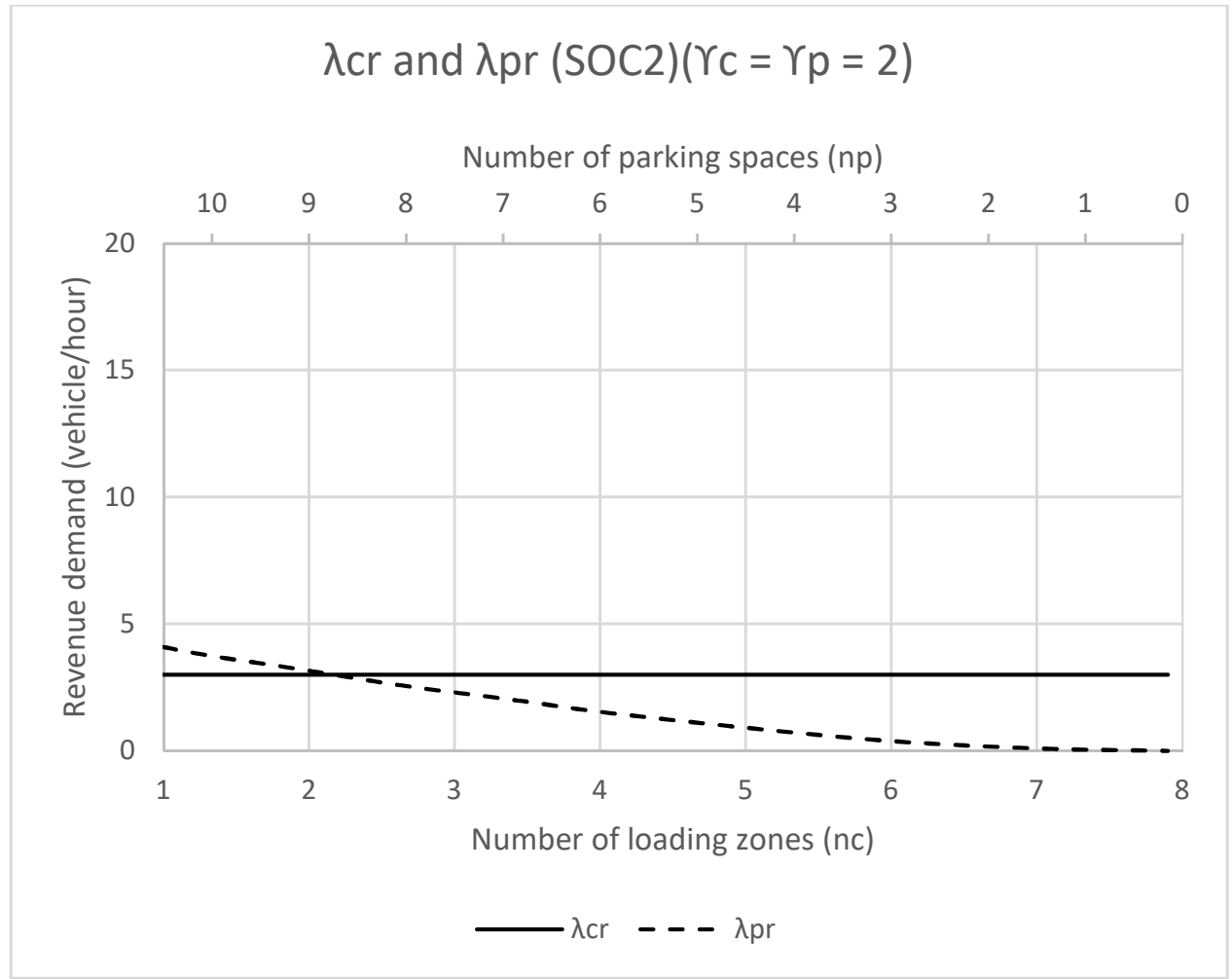

Figure 8.4 Revenue demand of scenario-2 social optimum model

Since the increase in the SOC from loading zones is higher than the decrease in the SOC from parking spaces, majority of curb length is allocated to loading zones, as shown in Figure 8.5. 


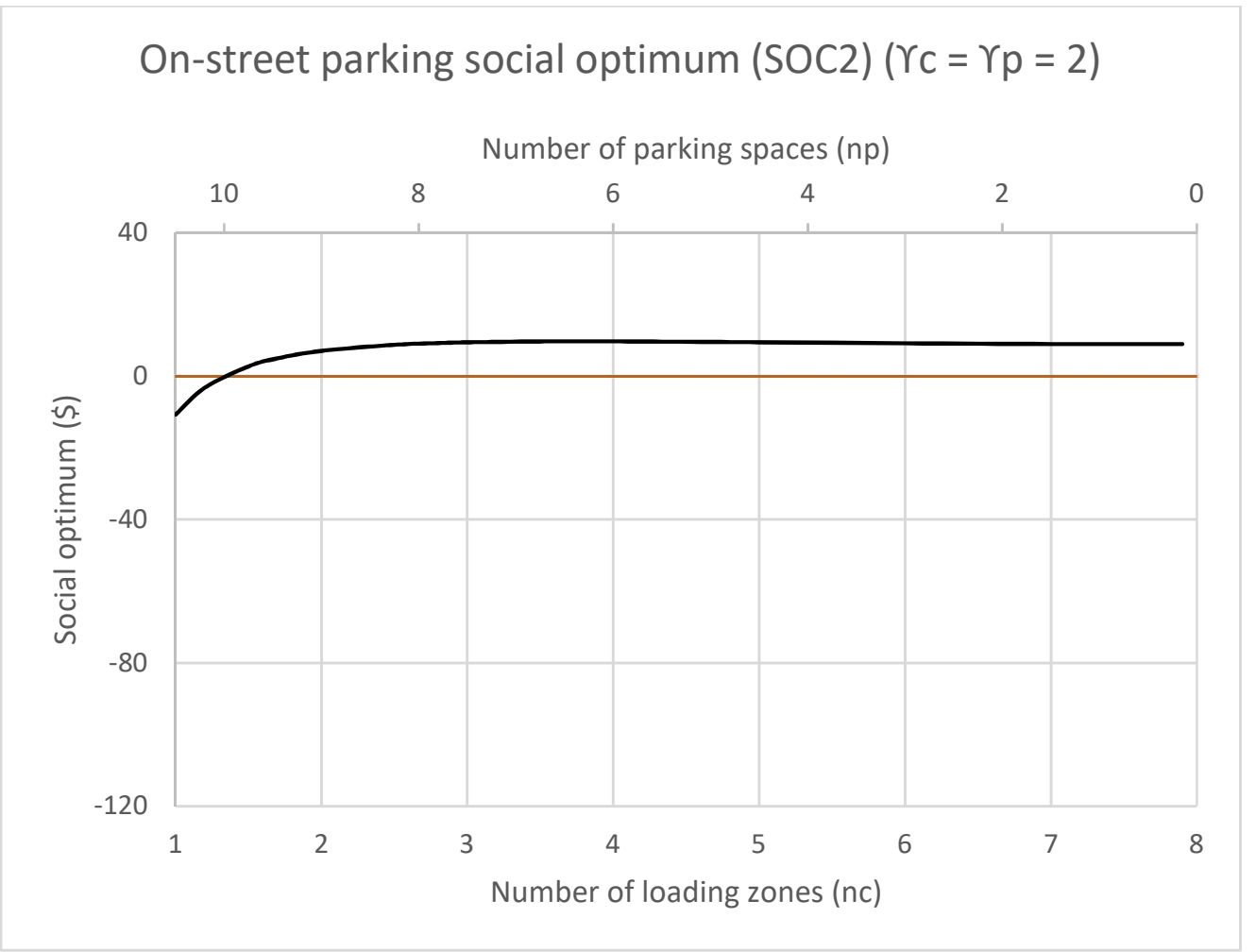

Figure 8.5 On-street scenario-2 social optimum

\subsubsection{Optimal solution for scenario-3 social optimum model}

The management strategy of scenario 3 is to let some commercial vehicles double park and to keep all passenger cars park on street. For loading zones, from $E q(7.17)$, if its curb allocation $\left(n_{c}\right)$ increases, the optimal meter rate $\left(c_{c o}^{*}\right)$ will decrease. However, the commercial revenue demand $\left(\lambda_{c r}\right)$ will increase. The SOC from loading zones will increase.

For parking spaces, the car revenue demand $\left(\lambda_{p r}\right)$ is constant. From $E q(7.18)$, if its allocation $\left(n_{p}\right)$ decreases, the optimal meter rate of parking spaces $\left(c_{p o}^{*}\right)$ decreases. The SOC from parking spaces will decrease.

Based on data from Table 6.1 and 6.2 , if the number of loading zones $\left(n_{c}\right)$ increases, $c_{c o}^{*}$ will decrease while $c_{p o}^{*}$ will decrease, as shown in Figure 8.6. $\lambda_{c r}$ will 
increase but $\lambda_{p r}$ will be constant, as shown in Figure 8.7. Since the increase in the SOC from loading zones is close to the decrease in the SOC from parking spaces, curb length is allocated to both parking types almost equally, as shown in Figure 8.8. When $n_{c}$ is 4.8 , the commercial revenue demand almost equals the arrival rate of commercial vehicles $\left(\lambda_{c}\right)$. If $n_{c}$ is more than 4.8 , all the commercial vehicles will park on street. Therefore, the maximum value of $n_{c}$ of scenario 3 is 4.8 .

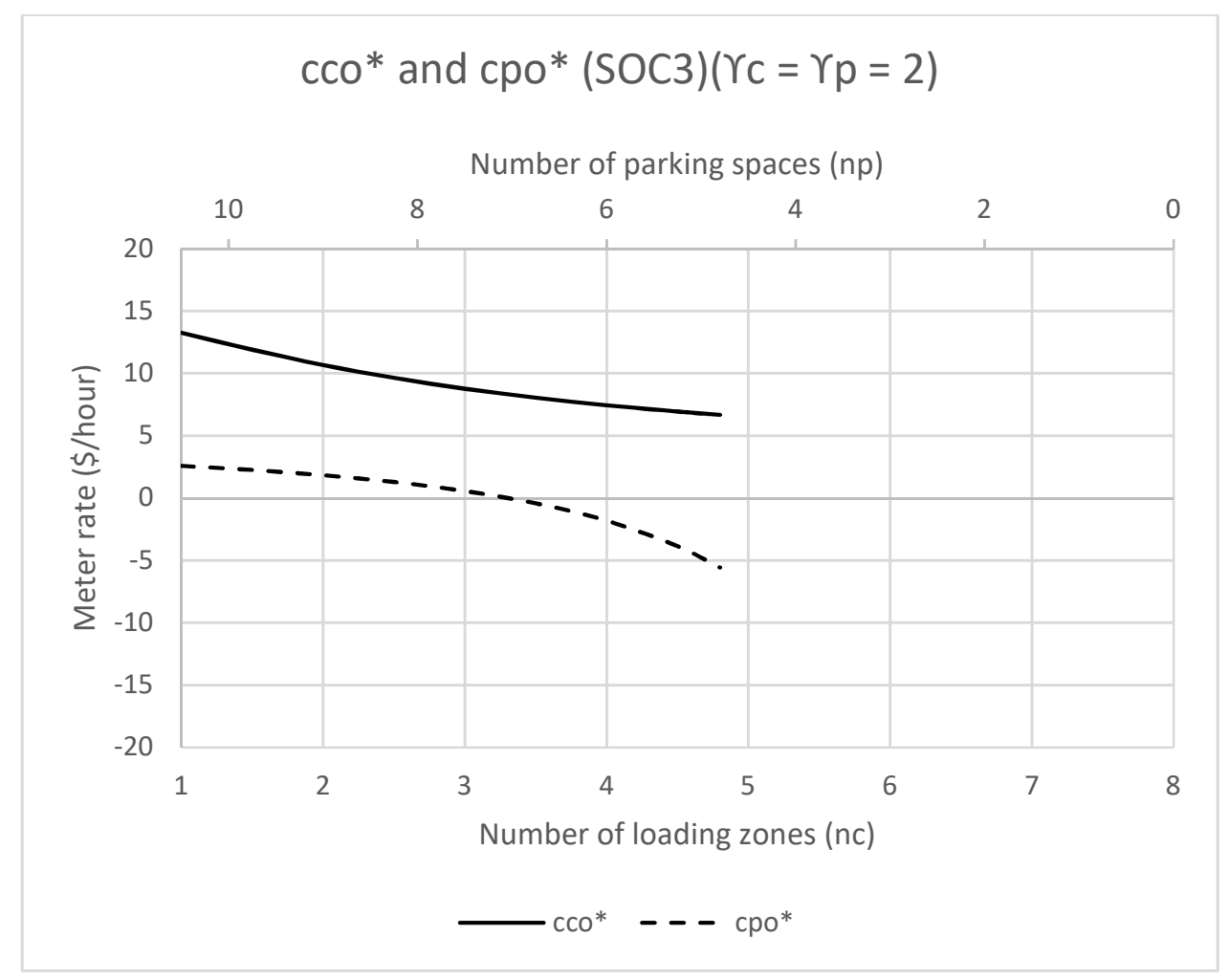

Figure 8.6 Optimal meter rates of scenario-3 social optimum model 


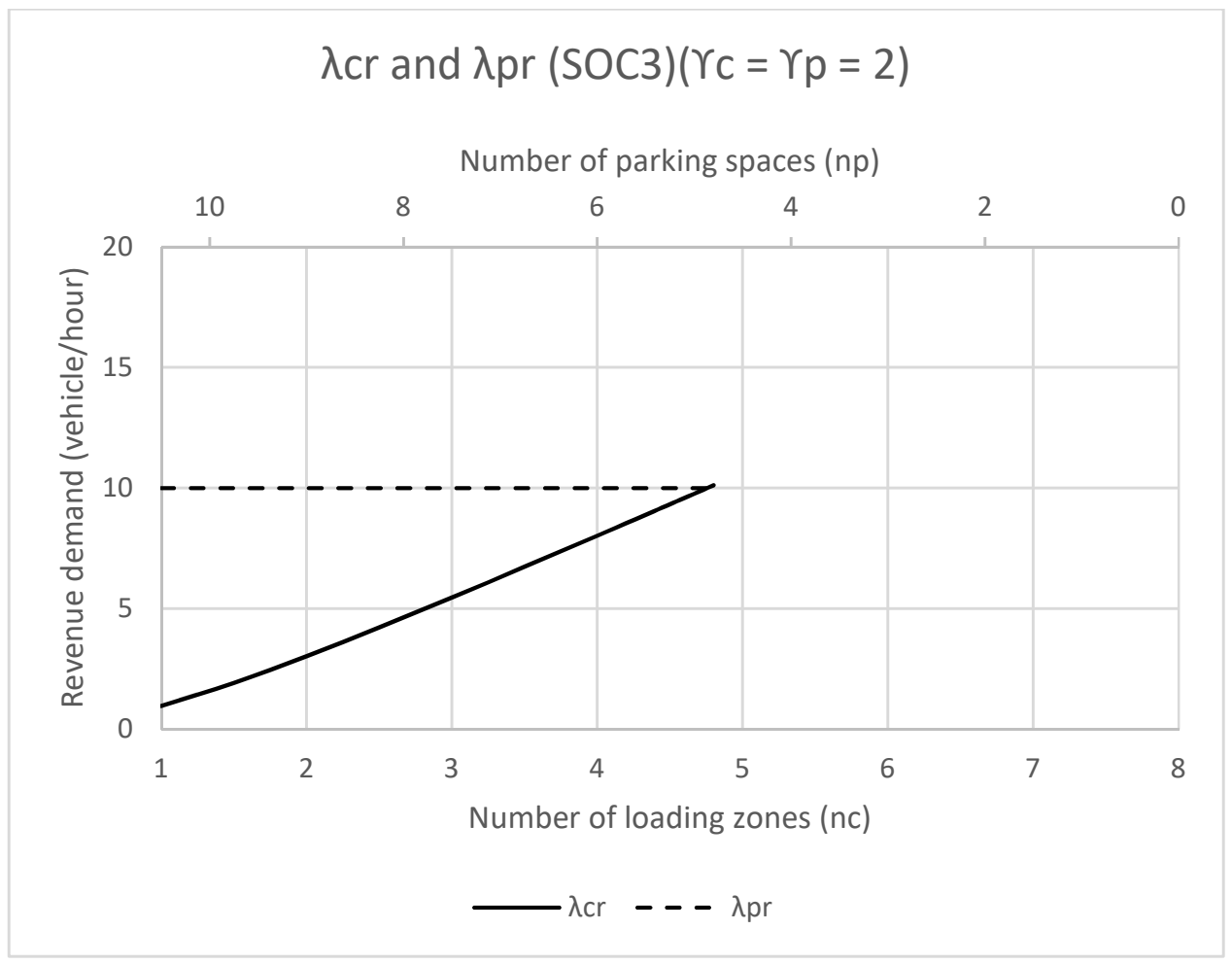

Figure 8.7 Revenue demand of scenario-3 social optimum model

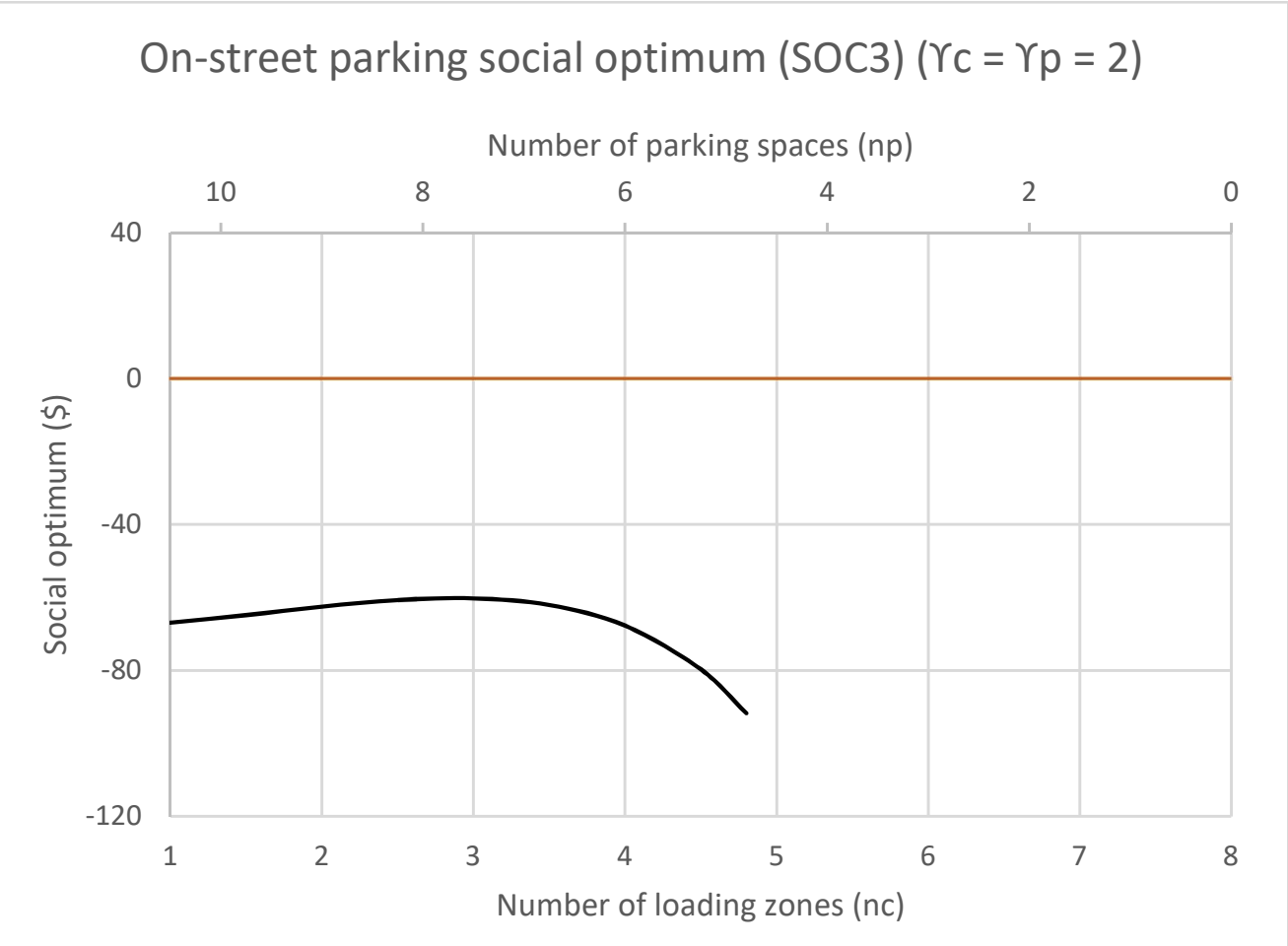

Figure 8.8 On-street scenario-3 social optimum 


\subsubsection{Optimal solution for scenario-4 social optimum model}

The management strategy of scenario 4 is to let some commercial vehicles double park and to let some cars park off street. For loading zones, from $E q(7.24)$, if its curb allocation increases $\left(n_{c}\right)$ the optimal meter rate of loading zones $\left(c_{c o}^{*}\right)$ will decrease. The commercial revenue demand will increase.

For parking spaces, from $E q(7.26)$, if its curb allocation $\left(n_{p}\right)$ decreases, the optimal meter rate of parking spaces $\left(c_{p o}^{*}\right)$ will increase. The car revenue demand will decrease.

Based on data from Table 6.1 and 6.2, if the number of loading zones $\left(n_{c}\right)$ increases, $c_{c o}^{*}$ will decrease while $c_{p o}^{*}$ will slightly increase, as shown in Figure 8.9. $\lambda_{c r}$ will increase but $\lambda_{p r}$ will decrease, as shown in Figure 8.10. When $n_{c}$ is 4.8 , the commercial revenue demand almost equals the arrival rate of commercial vehicles $\left(\lambda_{c}\right)$. If $n_{c}$ is more than 4.8 , all the commercial vehicles will park on street. Therefore, the maximum value of $n_{c}$ of scenario 4 is 4.8 . 


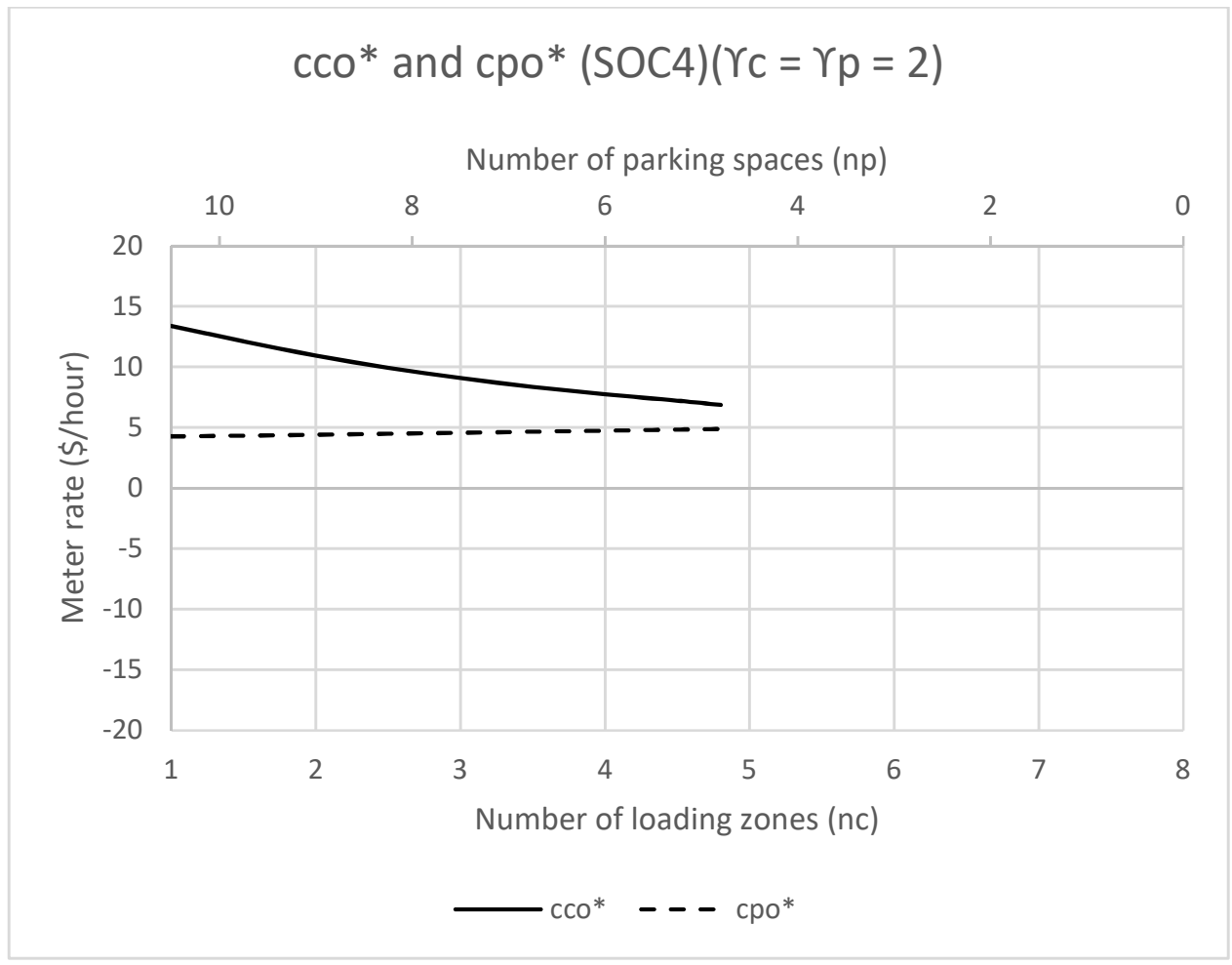

Figure 8.9 Optimal meter rates of scenario-4 social optimum model

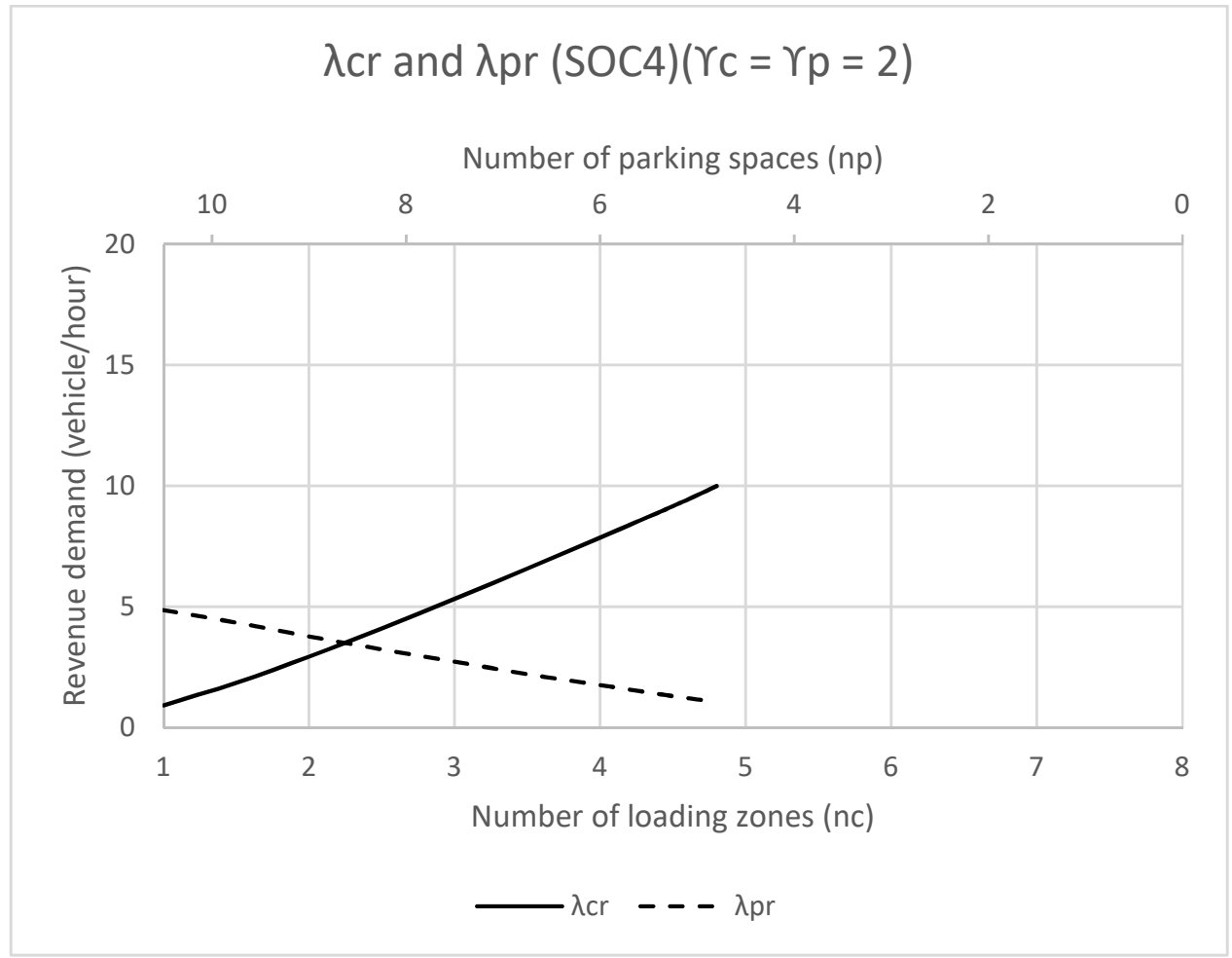

Figure 8.10 Revenue demand of scenario-4 social optimum model 136 
The increase in the commercial revenue demand is higher than the increase in the car revenue demand. Therefore, majority of curb length is allocated to loading zones, as shown in Figure 8.11.

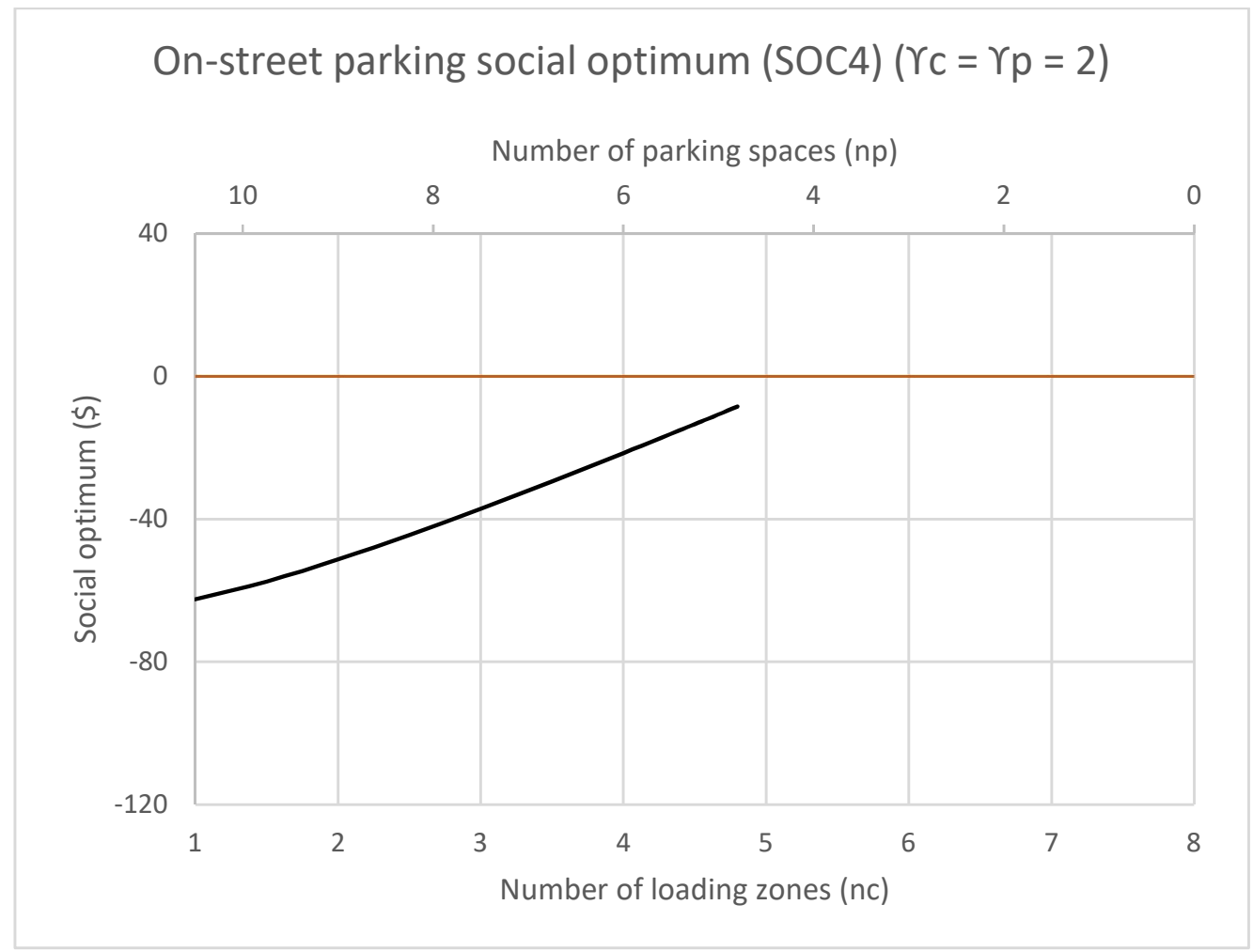

Figure 8.11 On-street scenario-4 social optimum

\section{$\underline{\text { 8.3.5 Optimal solution for social optimum models }}$}

From the optimal solutions of social optimum (SOC) models of all the scenarios, it is found that curb length allocation, meter rates, and revenue demand are different between scenarios, as shown in Table 8.3.

Compared with the optimal rates of parking spaces, the optimal meter rates of loading zones are very high. For commercial vehicles, the cost of the other option, double parking, is $\$ 15$. However, for cars, the cost of the other option, parking off street, is $\$ 5$. 
Table 8.3 Optimal solutions for the social optimum models $\left(\gamma_{c}=\gamma_{p}=2\right)$

\begin{tabular}{|l|r|r|r|r|}
\hline Scenario & 1 & 2 & 3 & 4 \\
\hline$\lambda_{c}$ & 3 & 3 & 10 & 10 \\
\hline$\lambda_{p}$ & 10 & 20 & 10 & 20 \\
\hline$n_{c}^{*}$ & 1.8 & 4.0 & 3.0 & 4.8 \\
\hline$n_{p}^{*}$ & 9.3 & 6.0 & 7.5 & 4.8 \\
\hline$c_{c o}^{*}$ & 9.79 & 13.95 & 8.77 & 6.87 \\
\hline$\lambda_{c r}$ & 3.00 & 3.00 & 5.47 & 10.00 \\
\hline$\lambda_{c}-\lambda_{c r}$ & & & 4.53 & 0.00 \\
\hline$c_{p o}^{*}$ & 2.18 & 4.84 & 0.56 & 4.88 \\
\hline$\lambda_{p r}$ & 10.00 & 1.54 & 10.00 & 1.06 \\
\hline revenue from loading zones & 7.34 & 10.46 & 11.99 & 17.17 \\
\hline revenue from parking spaces & 10.91 & 3.72 & 2.82 & 2.60 \\
\hline total revenue & 18.25 & 14.18 & 14.81 & 19.76 \\
\hline loading zones search cost & 3.91 & 0.79 & 15.58 & 20.33 \\
\hline parking space search cost & 14.09 & 1.60 & 21.67 & 1.20 \\
\hline commercial parking maneuver cost & 1.95 & 1.95 & 3.55 & 6.50 \\
\hline car parking maneuver cost & 1.30 & 0.20 & 1.30 & 0.14 \\
\hline double parking cost & 0.00 & 0.00 & 32.96 & 0.04 \\
\hline total social value & -2.99 & 9.64 & -60.25 & -8.43 \\
\hline
\end{tabular}

Compared with scenario 1 , the number of parking spaces of scenario 2 is lower although the passenger car arrival rate of scenario 2 is higher. For scenario 2, if parking spaces are congested, some car drivers will just park off street. The parking space meter rate of scenario 2 is not as low as the parking space meter rate of scenario 1 . The parking space meter rate of scenario 1 is low in order to keep the on-street option cheaper than the off-street option.

Compared with scenario 1, the number of loading zones of scenario 3 is higher and the meter rate of loading zones of scenario 3 is lower to order to keep as many commercial vehicles to park on street as possible. This way prevents high number of double-parking events, which increase much cost. Despite of necessity to have many loading zones, the number of parking spaces of scenario 3 cannot be too low since all cars park on street; 
otherwise, the meter rate of parking spaces will be very low. The meter rate of parking spaces of scenario 3 is lower since its curb length allocation is lower.

Compared with scenario 3, scenario 4 makes more social optimum. The number of loading zones of scenario 4 is high in order to prevent the high number of double-parking events. However, of scenario 3, the number of loading zones cannot be very high. This is because majority of curb length is allocated to parking spaces to prevent high parking search cost. As a result, the double-parking traffic delay cost of scenario 3 is very high, leading to very low social optimum.

\subsection{Impact of exponents of the parking search time function}

The parking search time function and how the exponents of the function affect the parking search cost and the revenue are already explained in Section 6.3. This section explains how the exponents affect the social optimum. From Chapter 7, the social optimum (SOC) is expressed as follow,

$$
\begin{aligned}
y_{s o c}=\frac{c_{c o} \lambda_{c r}}{\mu_{c}} & +\frac{c_{p o} \lambda_{p r}}{\mu_{p}}-c_{c t} \alpha_{w c}\left(\frac{\lambda_{c r}}{n_{c} \mu_{c}}\right)^{\gamma_{c}} \lambda_{c}-c_{d c} \lambda_{c r}-c_{d b}\left(\lambda_{c}-\lambda_{c r}\right) \\
& -\left[c_{p t}(1+\vartheta) \alpha_{w p}\left(\frac{\lambda_{p r}}{n_{p} \mu_{p}}\right)^{\gamma_{p}}+c_{p t} f_{d b} t_{s}\left(\lambda_{c}-\lambda_{c r}\right)\right] \lambda_{p}-c_{d p} \lambda_{p r}
\end{aligned}
$$

From Section 4.5,

$$
\begin{aligned}
& c_{c t} \alpha_{w c}\left(\frac{\lambda_{c r}}{n_{c} \mu_{c}}\right)^{\gamma_{c}}=\frac{1}{\mu_{c}}\left(\frac{c_{v} n_{e}}{\tau_{e}}-c_{c o}\right) \\
& c_{p t}(1+\vartheta) \alpha_{w p}\left(\frac{\lambda_{p r}}{n_{p} \mu_{p}}\right)^{\gamma_{p}}+c_{p t} f_{d b} t_{s}\left(\lambda_{c}-\lambda_{c}^{*}\right)=\frac{1}{\mu_{p}}\left(c_{p f}+c_{p f f} \mu_{p}-c_{p o}\right)
\end{aligned}
$$

Therefore, SOC becomes, 


$$
\begin{gathered}
y_{s o c}=\left(c_{c o}-c_{d c} \mu_{c}+c_{d b} \mu_{c}\right) \frac{\lambda_{c r}}{\mu_{c}}+c_{c o}\left(\frac{\lambda_{c}}{\mu_{c}}\right)+\left(c_{p o}-c_{d p} \mu_{p}\right) \frac{\lambda_{p r}}{\mu_{p}}+c_{p o}\left(\frac{\lambda_{p}}{\mu_{p}}\right) \\
-\left(\frac{c_{v} n_{e}}{\tau_{e}}\right) \frac{\lambda_{c}}{\mu_{c}}-c_{d b} \lambda_{c}-\left(c_{p f}+c_{p f f} \mu_{p}\right) \frac{\lambda_{p}}{\mu_{p}}
\end{gathered}
$$

From the equation above, SOC is positively related to meter rates $\left(c_{c o}, c_{p o}\right)$ and revenue demand $\left(\lambda_{c r}, \lambda_{p r}\right)$. This correlation has the same sign as the correlation of the revenue models.

The meter rates $c_{c o}$ and $c_{p o}$ are optimal which is estimated in Chapter 7 . The optimal meter rates are different between scenarios. In addition, the meter rates are a function of the exponents $\gamma_{c}$ and $\gamma_{p}$.

The revenue demand $\lambda_{c r}$ and $\lambda_{p r}$ are estimated in Section 4.5. Since the revenue demand is a function of the meter rates, it is a function of the exponents $\gamma_{c}$ and $\gamma_{p}$ and different between scenarios.

The estimation of the parking revenue is different between scenarios. It is required to analyze the impact of the exponents on the parking revenue for one scenario at a time.

\subsubsection{Impact of exponents on scenario-1 social optimum}

According to the optimal solution in Table 8.1, majority of curb length is allocated to parking spaces. From Section 8.2.1, the revenue is highly related to the meter rate of parking spaces $\left(c_{p o}\right)$. According to Section 7.7, the parking space meter rates of scenario 1 are expressed below,

$$
c_{p o}^{*}(S 1)=c_{p f}+c_{p f f} \mu_{p}-c_{p t}(1+\vartheta) \alpha_{w p} \mu_{p}\left(\frac{\lambda_{p}}{n_{p} \mu_{p}}\right)^{\gamma_{p}}
$$


From the formula above, if parking utilization of parking spaces $\left(\frac{\lambda_{p}}{n_{p} \mu_{p}}\right)$ is less than one, the higher the exponent, the higher the optimal meter rate, the higher the revenue. However, if the parking utilization is more than one, the higher the exponent, the lower the optimal meter rate, the lower the revenue, as shown in Figure 8.12.

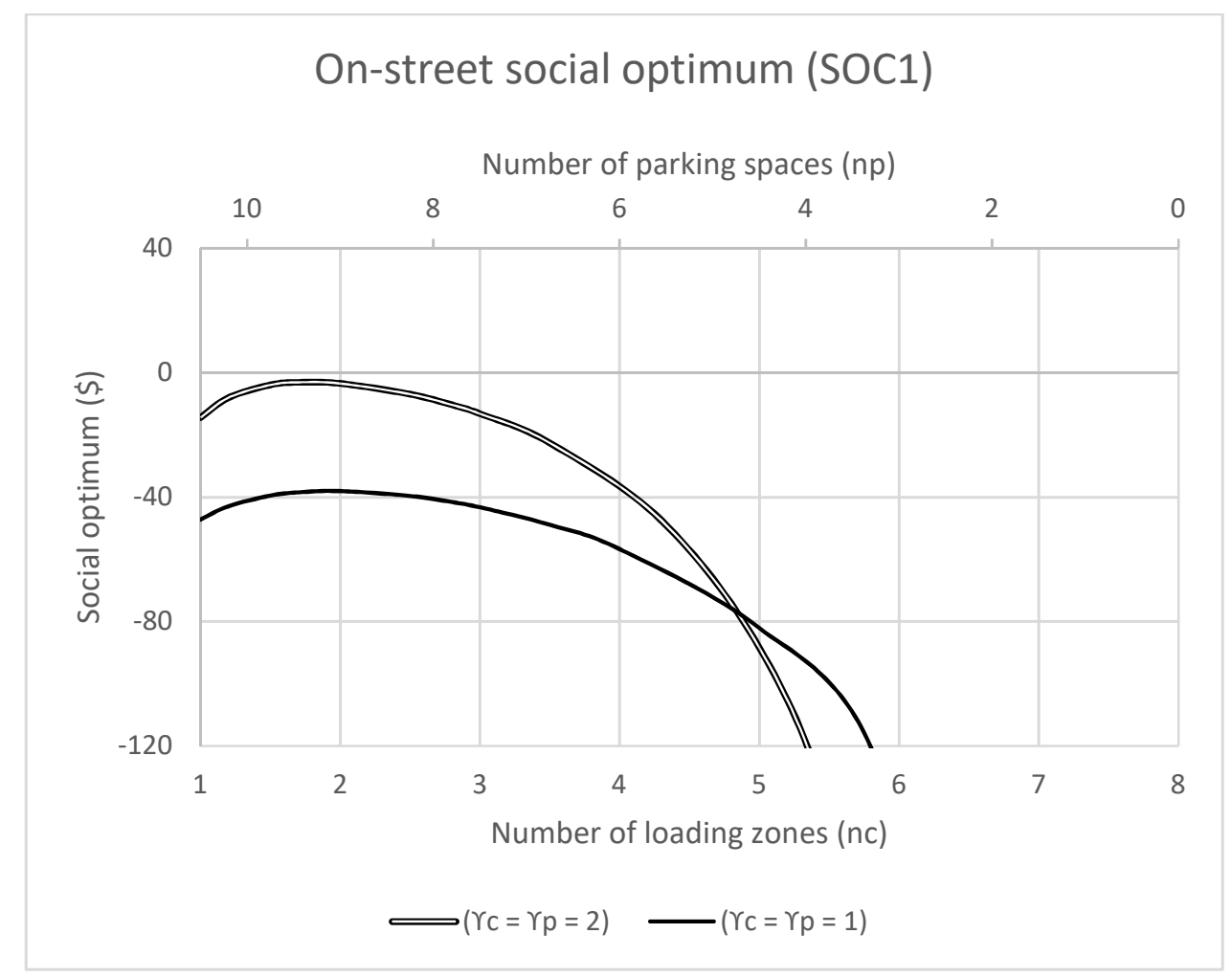

Figure 8.12 Impact of exponents on the scenario-1 social optimum

\subsubsection{Impact of exponents on scenario-2 social optimum}

From Section 8.2 , the social optimum is highly related to the meter rate of loading zones $\left(c_{c o}\right)$. According to Section 7.7, the loading zones meter rates of scenario 2 are expressed as follow,

$c_{c o}^{*}(S 2)=\frac{c_{v} n_{e}}{\tau_{e}}-c_{c t} \alpha_{w c} \mu_{c}\left(\frac{\lambda_{c}}{n_{c} \mu_{c}}\right)^{\gamma_{c}}$ 
From the formula above, the higher the exponent, the higher the meter rate, the higher the revenue, as shown in Figure 8.13. From Section 7.2.2, the more the number of loading zones $\left(n_{c}\right)$, the less the car revenue demand $\left(\lambda_{p r}\right)$, the less the difference between the revenue of $\left(\gamma_{c}=\gamma_{p}=2\right)$ and the revenue of $\left(\gamma_{c}=\gamma_{p}=1\right)$.

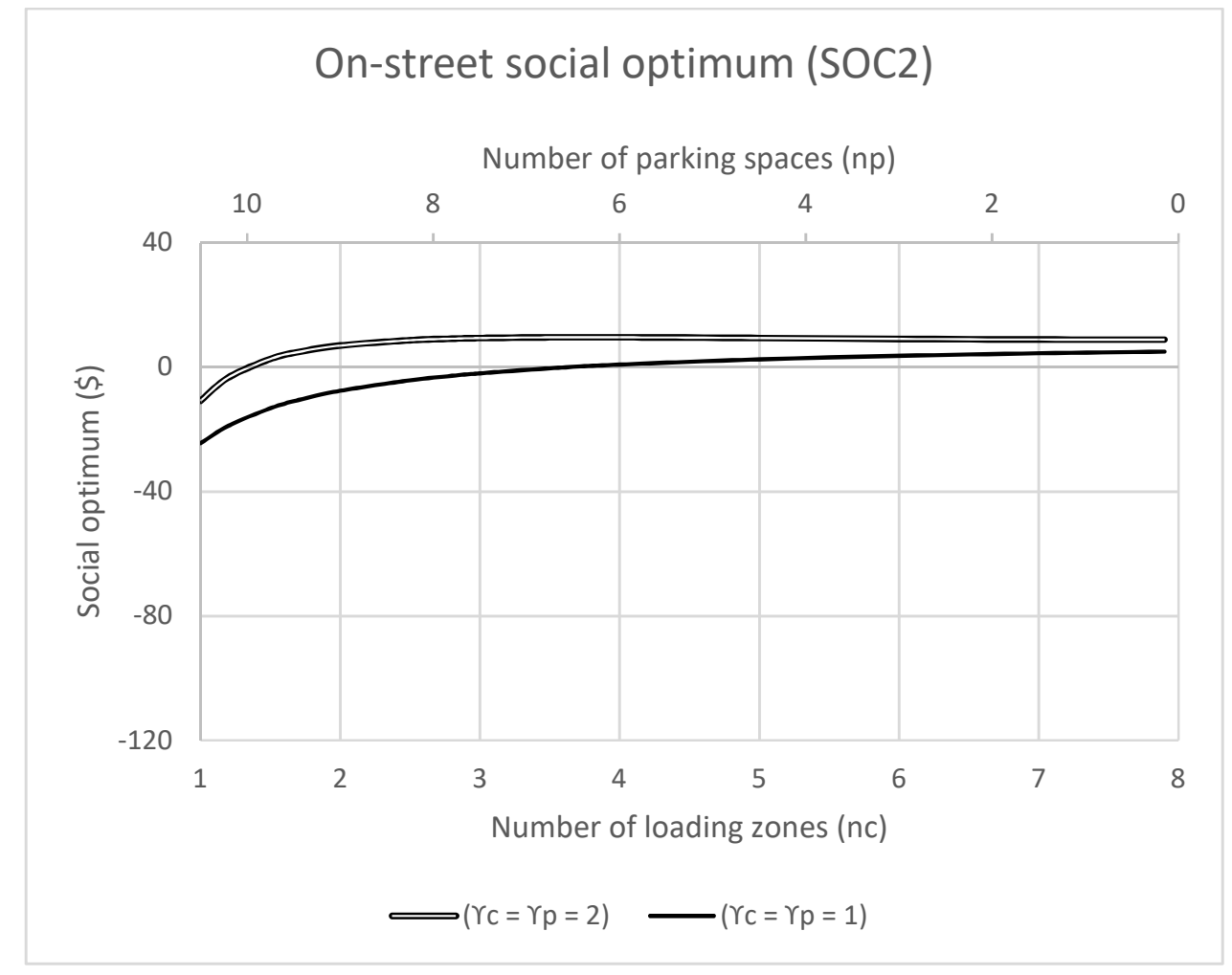

Figure 8.13 Impact of exponents on the scenario-2 social optimum

\section{$\underline{\text { 8.4.3 Impact of exponents on scenario-3 social optimum }}$}

According to Section 7.7, the meter rates of scenario 3 are expressed as follow,

$$
\begin{aligned}
c_{c o}^{*}(S 3)=\frac{\gamma_{c} c_{v} n_{e}}{\left(\gamma_{c}+1\right) \tau_{e}}+\frac{\left(c_{d c} \mu_{c}-c_{d b} \mu_{c}-c_{p t} f_{d b} t_{s} \lambda_{p} \mu_{c}\right)}{\left(\gamma_{c}+1\right)} \\
+\frac{\gamma_{c}}{\left(\gamma_{c}+1\right)}\left(c_{c t} \alpha_{w c} \mu_{c}\right)^{\frac{1}{\gamma_{c}}}\left[\frac{c_{v} n_{e}}{\tau_{e}}-c_{c o}^{*}(S 3)\right]^{\frac{\gamma_{c}-1}{\gamma_{c}}}\left(\frac{\lambda_{c}}{n_{c} \mu_{c}}\right)
\end{aligned}
$$




$$
\begin{aligned}
c_{p o}^{*}(S 3)=c_{p f} & +c_{p f f} \mu_{p}-c_{p t}(1+\vartheta) \alpha_{w p} \mu_{p}\left(\frac{\lambda_{p}}{n_{p} \mu_{p}}\right)^{\gamma_{p}} \\
& -c_{p t} \mu_{p} f_{d b} t_{s}\left[\lambda_{c}-n_{c} \mu_{c}\left(\frac{c_{v} n_{e}-c_{c o} \tau_{e}}{c_{c t} \alpha_{w c} \mu_{c} \tau_{e}}\right)^{\frac{1}{\gamma_{c}}}\right]
\end{aligned}
$$

From the formula for the optimal meter rate of parking spaces $\left(c_{c o}^{*}\right)$, the higher the exponents, the higher the meter rate. This leads to higher SOC. From the formula for the optimal meter rate of parking spaces $\left(c_{p o}^{*}\right)$, if the exponents are higher, either low number of parking spaces $\left(n_{p}\right)$ or low number of load zones $\left(n_{c}\right)$ will lead to lower $c_{p o}^{*}$. This reduces the difference between the revenue of $\left(\gamma_{c}=\gamma_{p}=2\right)$ and the revenue of $\left(\gamma_{c}=\gamma_{p}=1\right)$ when $n_{p}$ or $n_{c}$ is low, as shown in Figure 8.14.

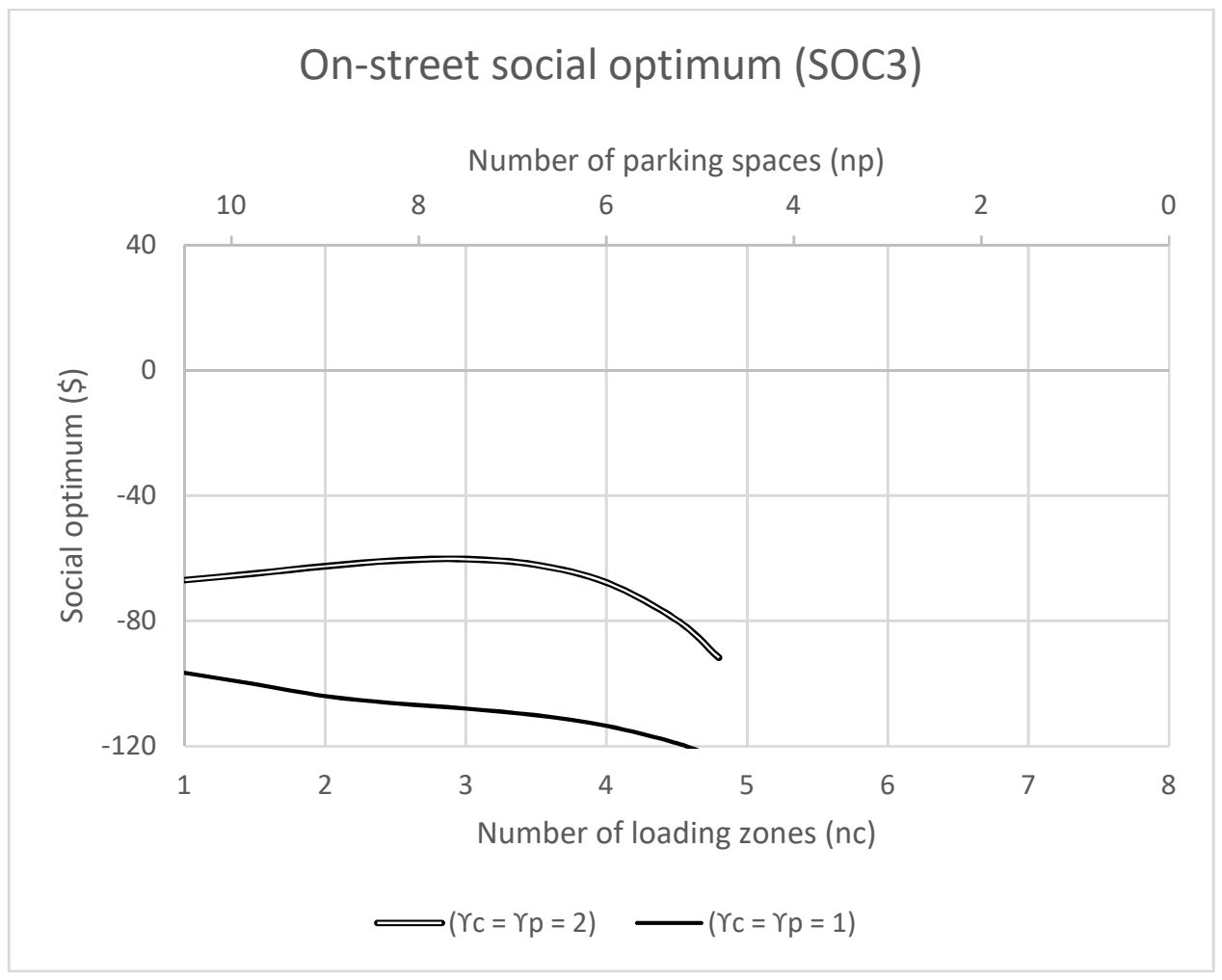

Figure 8.14 Impact of exponents on the scenario-3 social optimum 


\section{$\underline{\text { 8.4.4 Impact of exponents on scenario-4 social optimum }}$}

According to Section 7.7, the formula of the optimal meter rates of scenario 4 are complicated. It is much easier to show the numerical results of the meter rates which are shown in Figure 8.15. From Section 8.2.4, majority of curb length is allocated to loading zones. The optimal meter rate $c_{c o}^{*}$ of $\left(\gamma_{c}=\gamma_{p}=2\right)$ is higher than the optimal meter rate $c_{c o}^{*}$ of $\left(\gamma_{c}=\gamma_{p}=1\right)$ except when parking utilization is high $\left(n_{c}\right.$ is small). This leads to higher SOC when parking utilization is not high, as shown in Figure 8.16.

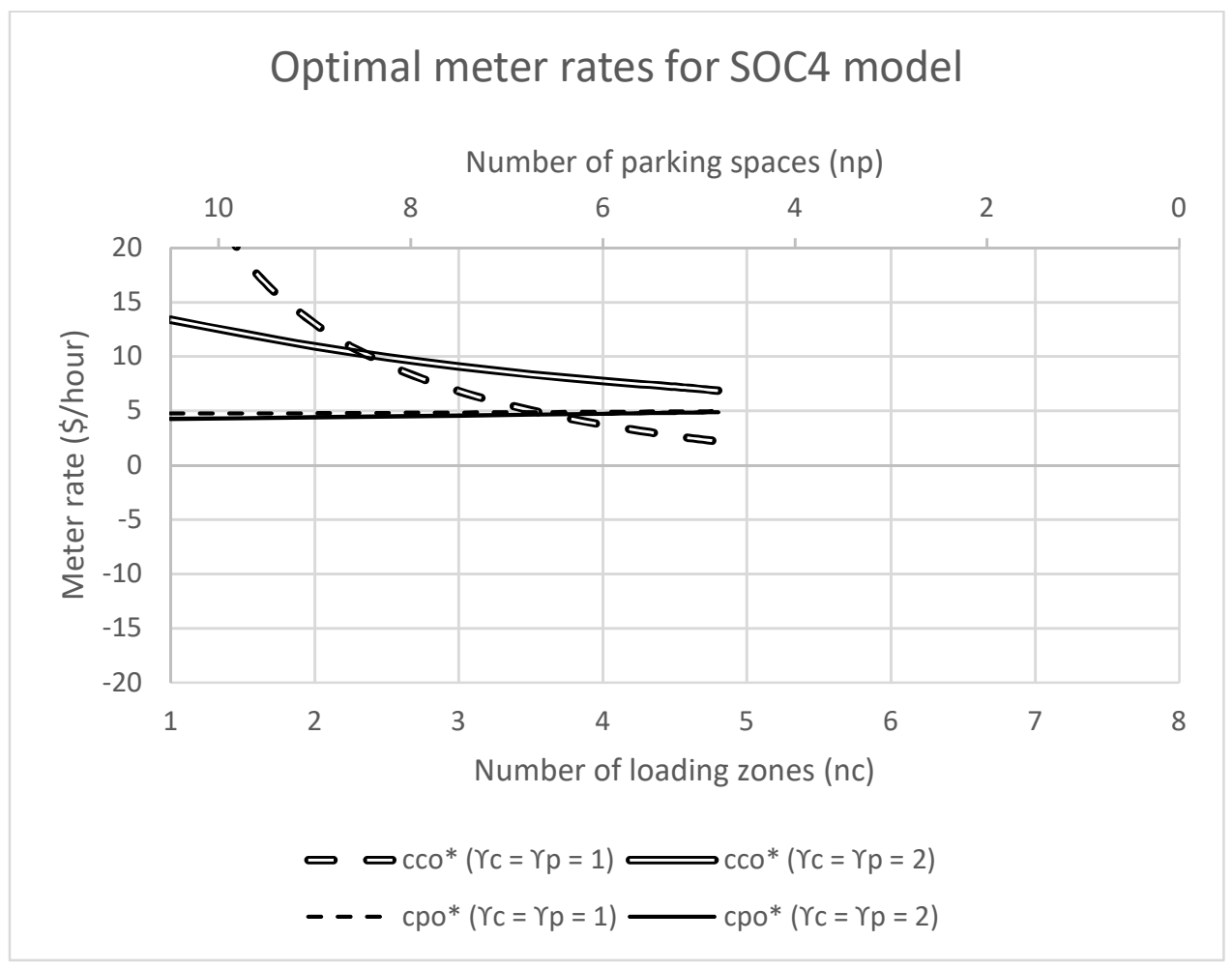

Figure 8.15 Optimal rates for scenario-4 SOC models from different exponents 


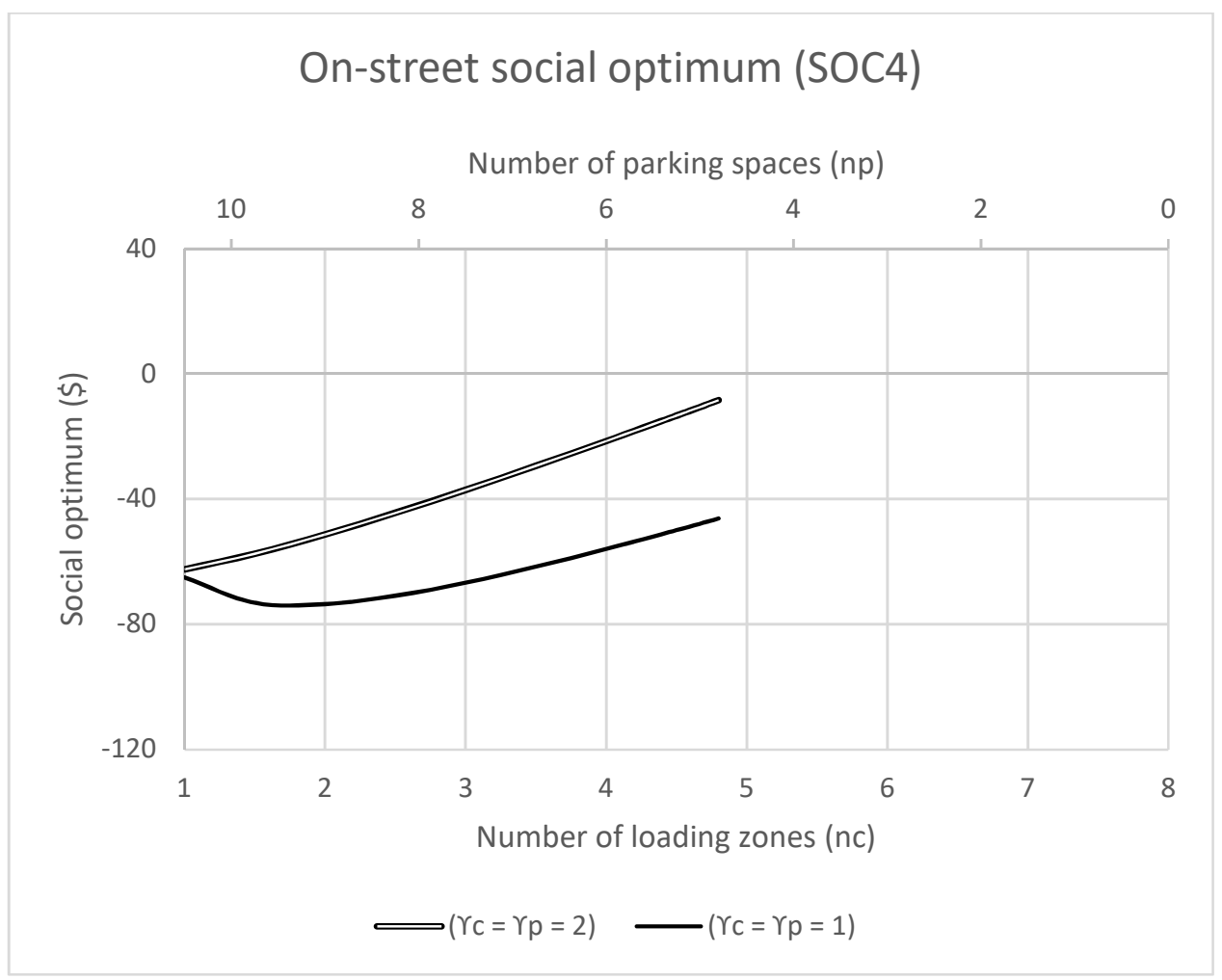

Figure 8.16 Impact of exponents on the social optimum for scenario 4

\subsection{Compare the revenue models with the social optimum models}

The first comparison is values of revenue and social optimum (SOC) from both the models. From Table 6-5 and Table 8.1, revenues from the revenue models are higher than revenues from the SOC models. Similarly, SOCs from the SOC models are higher than SOCs from the revenue models. This difference reflects the different objective functions from these models.

The second comparison is optimal meter rates of both the models. Of scenario 1, the rates are the same since both the objective functions are a linear function of meter rates. The constraint on the meter rates give the optimal solution.

Of scenario 2 , the meter rates of loading zones are the same since the objective functions are a linear function of the meter rates. The meter rates of parking spaces are 
different because the rates of the SOC models are higher. The SOC models take into account all the costs. The higher the meter rate, the lower the vehicles park on street, the lower the parking search cost, as shown in Figure 8.17.

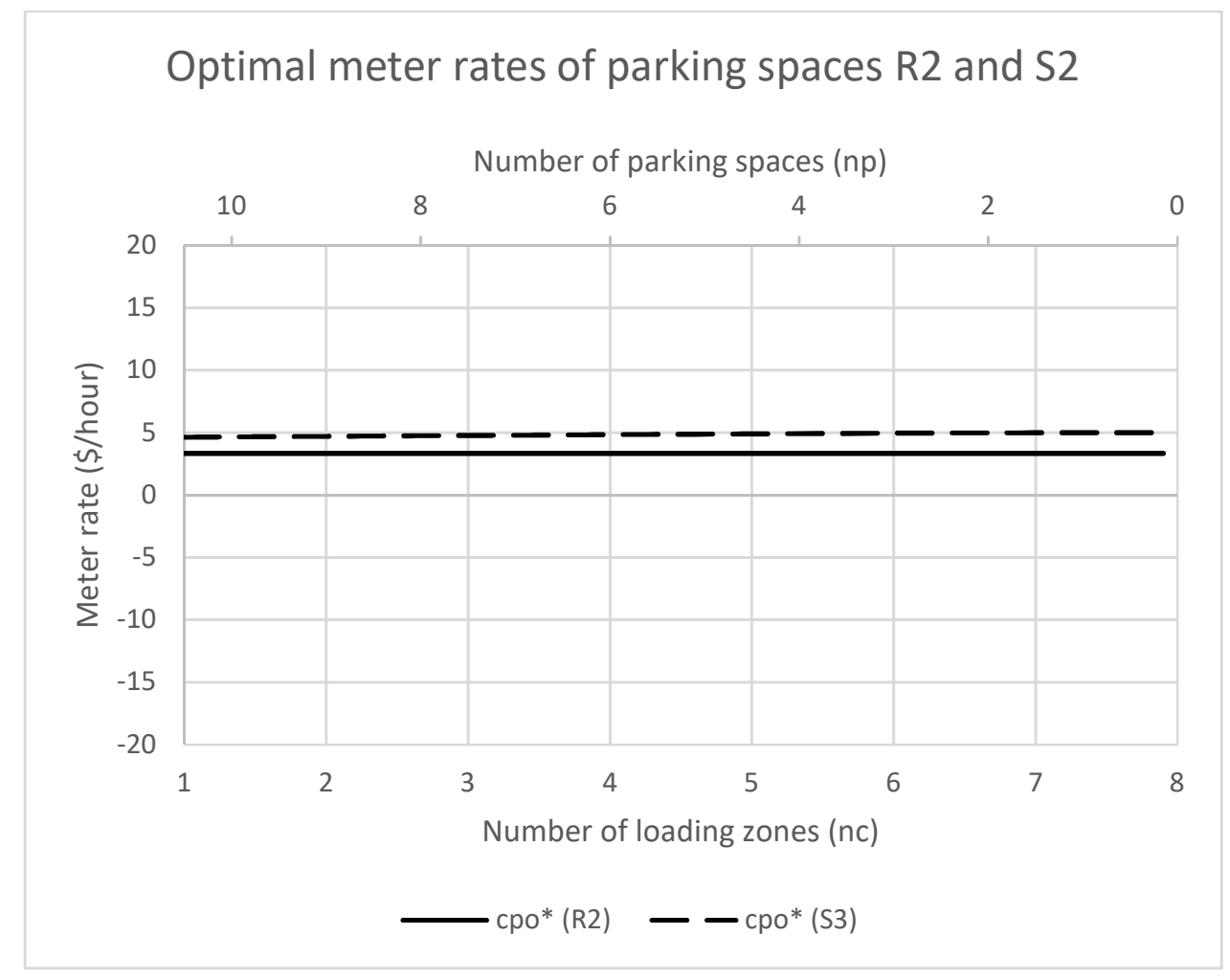

Figure 8.17 Difference between $c_{p o}^{*}$ of revenue and social optimum models

Of scenario 3 , the meter rates of parking spaces are the same since the objective functions are a linear function of the meter rates. The loading zone meter rates of both the models are different. The meter rate of the revenue model is constant while the meter rate of SOC model is negatively related to the number of loading zones since the parking search cost is negatively related to the number of loading zones, as shown in Figure 8.18. 


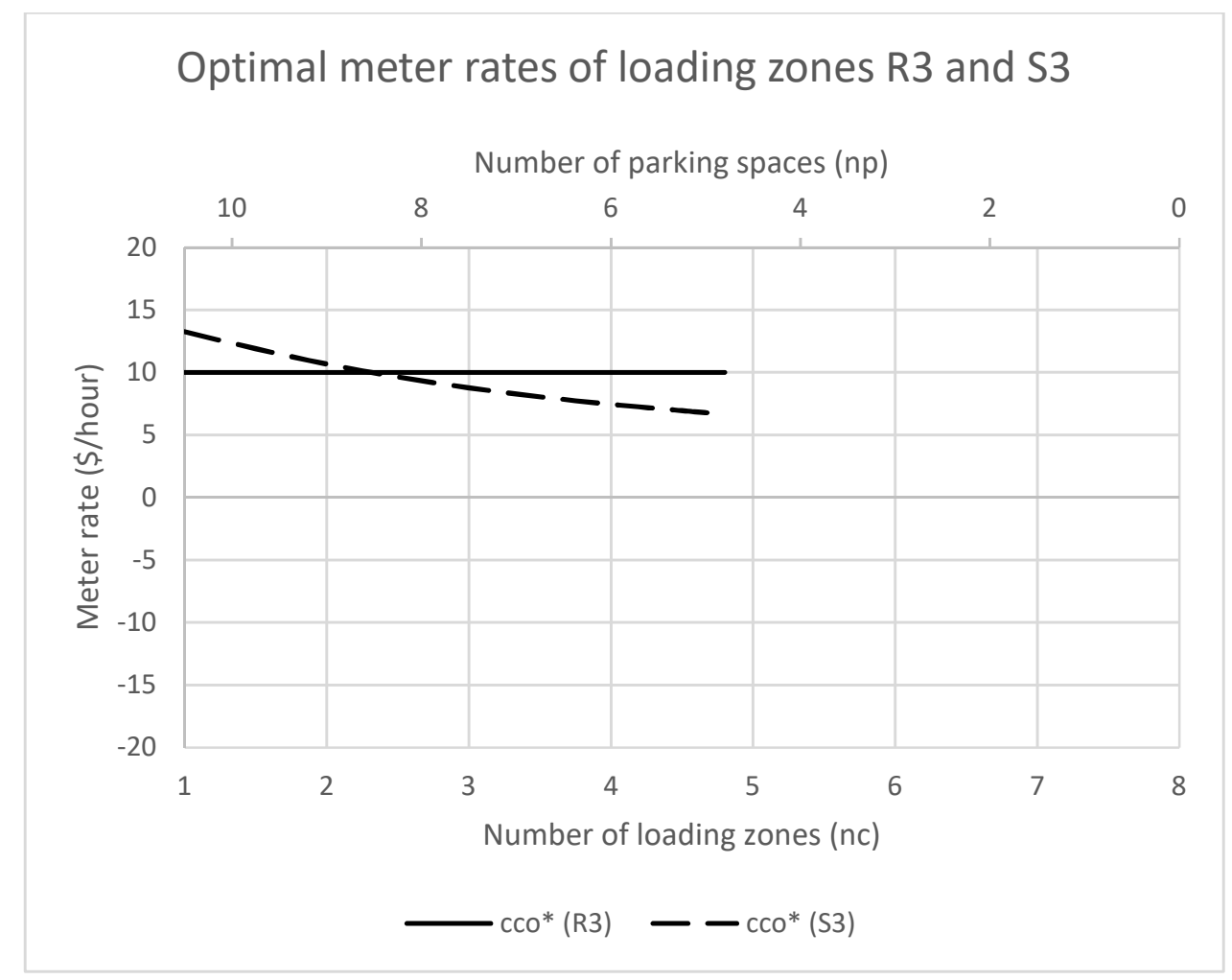

Figure 8.18 Difference between $c_{c o}^{*}$ of revenue and social optimum models

Of scenario 4, the meter rates of both parking types are different. The meter rates of parking spaces are different in the same way as described in scenario 2 . The meter rates of loading zones are different in the same way as described in scenario 3.

The optimal solutions of the SOC models are also different from the optimal solution of the revenue models. For scenario 2 , the meter of parking space $\left(c_{p o}^{*}\right)$ of the SOC model is higher than $c_{p o}^{*}$ of the revenue model. The high $c_{p o}^{*}$ of the SOC model reduces the number of passenger vehicles parking on street so does the parking search cost. For scenarios 3 and 4 , the meter rates of loading zones $\left(c_{c o}^{*}\right)$ of the SOC models is lower than $c_{c o}^{*}$ of the revenue models. This way reduces the number of double-parking events, as shown in Table 8.4 . 
Table 8.4 Comparison of optimal solutions of revenue and SOC models

\begin{tabular}{|}
\begin{tabular}{|r|r|r|r|r|r|r|r|r|r|r|r|}
\hline Scenario & $\lambda_{c}$ & $\lambda_{p}$ & $n_{c}^{*}$ & $n_{p}^{*}$ & $c_{c o}^{*}$ & $\lambda_{c r}$ & $\lambda_{c}-\lambda_{c r}$ & $c_{p o}^{*}$ & $\lambda_{p r}$ & revenue & SOC \\
\hline \\
Revenue models \\
\hline 2 & 3 & 20 & 2.5 & 8.3 & 12.30 & 3.00 & - & 3.33 & 6.82 & 20.59 & -1.93 \\
\hline 3 & 10 & 10 & 1.5 & 9.8 & 10.00 & 2.45 & 7.55 & 2.26 & 10.00 & 17.44 & -65.69 \\
\hline 4 & 10 & 20 & 6.0 & 3.0 & 9.95 & 9.85 & 0.15 & 3.33 & 2.48 & 28.62 & -8.49 \\
\hline \multicolumn{10}{|l|}{ SOC models } \\
\hline 2 & 3 & 20 & 4.0 & 6.0 & 13.95 & 3.00 & 0.00 & 4.84 & 1.54 & 14.18 & 9.64 \\
\hline 3 & 10 & 10 & 3.0 & 7.5 & 8.86 & 5.43 & 4.57 & 0.56 & 10.00 & 14.81 & -60.25 \\
\hline 4 & 10 & 20 & 4.8 & 4.8 & 6.87 & 10.00 & 0.00 & 1.06 & 1.06 & 19.76 & -8.43 \\
\hline
\end{tabular}
\end{tabular}

\subsection{Conclusion}

This chapter demonstrates how the social optimum (SOC) maximization models optimize the meter rates and space allocation. Since the SOC models are developed based on four scenarios, there are four set of the data: one set for one scenario. The main difference between scenarios is arrival rates of parking commercial vehicles and parking passenger cars.

Of scenario 1 (parking on street only), the revenue demand (how many vehicles park on street) is constant. The meter rates of loading zones and parking spaces are positively related to curb length allocation. The less the curb allocation, the lower the meter rate, the lower the revenue. Majority of curb length is allocated to a parking type of which meter rate is more positively related to the allocation. 
Compared with scenario 2 (on-street and off-street are chosen), the SOC of scenario 1 is lower. For scenario 2 , if parking spaces are congested, some car drivers will just park off street. This leads to lower parking search cost and higher SOC. The parking space meter rate of scenario 2 is higher than the parking space meter rate of scenario 1 . The parking space meter rate of scenario 1 is low in order to keep the on-street option cheaper than the off-street option.

Of scenario 4 (all parking options chosen), meter rates of loading zones and parking spaces are negatively related to curb length allocation. The lower the curb allocation, the higher the meter rate to push more drivers choose other options rather than parking on street which prevents high parking search cost and low SOC. For each parking type, its revenue demand is positively related to its curb allocation. The more the curb allocation, the more the revenue demand, the more the revenue. Majority of curb is allocated to loading zones in order to prevent double-parking events.

Compared with scenario 4, passenger cars of scenario 3 park on street only. Therefore, the number of parking spaces of scenario 3 is higher in order to prevent high parking search cost of passenger cars. Then, the number of loading zones of scenario 3 is lower. That leads to more double-parking events which increases cost substantially and decreases SOC substantially.

The exponents of the parking search time function affect the revenue and the social optimum (SOC). The higher the exponents, the higher the revenue, the higher the SOC. There is one exception where all the vehicles park on street only. If parking utilization is high, the optimal meter rate of the higher exponent will be less than the optimal meter rate 
of the lower exponent. This leads to the SOC of higher exponent less than the SOC of lower exponent.

Compared with the revenue models, the meter rates are equal when vehicles park on street only. However, the meter rates are different when vehicles park on street and the other option. The meter rates of the revenue models are constant while the meter rates of the SOC models are negatively related to curb length allocation. Moreover, for parking spaces, the meter rate of SOC models is higher in order to push more vehicles not to park on street which reduces the parking search cost and increases the social optimum. 


\section{Chapter 9 Conclusion}

This conclusion summarizes the findings and contributions from the research questions. Limitations and future directions are also discussed.

\subsection{Findings and contributions}

9.1.1 Maximize revenue and social optimum of on-street parking system

The revenue and social optimum of the on-street parking system are maximized with optimization models. The on-street parking system includes loading zones and parking spaces. The users are commercial vehicles and passenger cars. There are two options for each vehicle type. Commercial vehicles can park on street and double park. Passenger cars can park on street and off street. The decision variables are curb length allocation to each parking type and its meter rate. The social optimum equals revenue from on-street parking minus costs to drivers and costs due to traffic delays.

The optimal results from case studies demonstrate how the arrival rate of parking vehicles affects the meter rate. If the arrival rate is low, compared with parking supply, all vehicles will park on street. The meter rate depends on the arrival rate. As the arrival rate increases, the parking search cost will increase. To keep the on-street option cheaper than the other parking option, the meter rate has to decrease. However, if the arrival rate is high, compared with parking supply, some drivers will park on street while others will choose the other parking option. The meter rate usually does not depend on the arrival rate.

The optimal results from case studies also demonstrate how the arrival rates of parking vehicles affect curb length allocation. If the arrival rates are low, much curb length will be allocated to a parking type of which meter rate is the most related to the allocation. However, if the arrival rates are high, much curb length will be allocated to a parking type 
of which revenue demand is the most related to the allocation. In practice, though, the meter rate usually does not depend on the allocation. Compared with the revenue model, the social optimum model allocates higher curb length to a parking type of which counterpart causes more externalities. For example, a commercial vehicle double parking causes a lot of traffic delay costs. The SOC model allocates higher curb length to loading zones than the revenue model does.

The parking optimization models can be applied to current parking tools and current vehicle types although the models are developed based on only curb length allocation and meter rate. There are other parking management tools for on-street parking utilizing Intelligent Transportation System (ITS). For example, closed circuit Televisions (CCTVs) can collect the real-time data about parking occupancy and the data is sent to a parking reservation system. Using these tools, it is possible to set real-time curb length allocation and meter rates. Change in curb length and meter rate can be displayed by variable message signs.

In the future, vehicle sizes might change, or become zero if commercial vehicles are freight tricycles. For example, freight tricycles in Portland, Oregon can park on sidewalks (Tipagornwong and Figliozzi, 2014). The change in vehicle sizes can change arrival rate, service rate, value of time, and other variables as well. These changes are the model inputs. Therefore, the proposed parking models can deal with the change of vehicle sizes and types.

In the literature, models for both vehicle types only optimize either curb length allocation or meter rate for both vehicle types. The models of this study optimize both curb length allocation and meter rate. In addition, the models take interaction between loading 
zones and parking spaces into account. If loading zones are highly occupied, some commercial vehicles will double park, which blocks a traffic lane. This results in delays to through traffic and car drivers looking for available parking spaces.

9.1.2 Estimation of traffic delays due to a double-parking event

This study takes traffic signals and intersections adjacent to a double-parking event into account. It shows that a traffic signal at the upstream intersection affects traffic delays caused by the double-parking event because the traffic signal changes distribution of traffic flow rates. In particular, the traffic delays will increase if a double-parking event starts while traffic approaches the event with saturation flow rate.

In the literature, either an intersection upstream of the event or an intersection downstream of the event is considered. However, no studies take into account both intersections at the same time.

\subsection{Limitations}

\subsubsection{Assumption on parking optimization models}

The main assumption of the models in this study is based on a cost breakeven condition. The breakeven condition is where the costs of two parking options are equal. In some scenarios, if the arrival rate increases, the meter rate will decrease to keep the costs still equal. This assumption may not follow a demand and supply relationship, stating that higher arrival rate leads to higher meter rates.

\subsubsection{Inequity of pricing meter rates}

The optimal meter rates from the parking models are very different between loading zones and parking spaces. Therefore, drivers of commercial vehicles and drivers of 
passenger cars pay different fees. This difference in meter rates can cause inequity between different driver types and be hard to implement in practice.

\subsection{Future directions}

9.3.1 Alternate vehicles for truck companies

Commercial vehicles park before serving customers. Some vehicle types such as freight tricycles do not need to park on street. Freight tricycles can park on curb in some cities. Delivering goods by freight tricycles can reduce the arrival rate of commercial vehicles (Tipagornwong and Figliozzi, 2014).

\subsubsection{Another parking violation}

Commercial vehicles are not the only vehicles to violate parking regulations by double parking, as passenger cars also violate parking regulations. When parking spaces are full, passenger cars sometimes park in loading zones which are designated for commercial vehicles only (Simon and Conway, 2019; Dey et. al.,2019). This reduces availability of loading zones that causes more commercial vehicles to double park.

\subsubsection{Heterogenous commercial vehicles}

Commercial vehicles deliver various sizes of products, including wholesale food, small boxes of food, clothes, pharmaceutical products, and smaller packages. This can change several variables including value of time, vehicle type, and parking duration. Since the expected double-parking cost is a decreasing function of parking duration, commercial vehicles with a short parking duration is likely to double park while commercial vehicles with long parking duration are likely to search for available loading zones (Figliozzi and Tipagornwong, 2017). The variation in values of variables can change optimal solutions for the parking models. 


\subsubsection{Another user of curb length}

Another curb user is transit such as buses. To make the parking models more versatile, the transit users need to be included in the parking optimization models. To incorporate the transit users in the models, the base data has to be available, similar to commercial vehicles and passenger cars, for example arrival rate, service rate, and unit parking length. 


\section{Reference}

Aiura, N. \& Taniguchi, E. (2006). "Planning On-Street Loading-Unloading Spaces Considering the Behaviour of Pickup-Delivery Vehicles and Parking Enforcement." Langkawi (Malaysia), Elsevier, pp. 107-119.

Alho, A. R. \& e Silva, J. D. A. (2015). "Utilizing Urban Form Characteristics in Urban Logistics Analysis: A Case Study in Lisbon." Journal of Transport Geography, Volume 42, pp. 57-71.

Alho, A. R., e Silva, J. D. A., de Sousa, J. P. \& Blanco, E. (2016). "Decreasing Congestion by Optimizing the Number, Location, and Usage of Loading/Unloading Bays for Urban Freight." paper presented to the Transportation Research Board 95th Annual Meeting, Washington, D.C., 10-14 January.

Allen, J., Anderson, S., Browne, M. \& Jones, P. (2000). "A framework for considering policies to encourage sustainable urban freight traffic and goods/service flows." [Online] Available at: http://home.wmin.ac.uk/transport/projects/u-d-summ.htm (Accessed October 2014).

Amer, A. \& Chow, J. (2017). "A Downtown On-Street Parking Model with Urban Truck Delivery." Transportation Research Part A: Policy and Practice, Volume 102, Pages 51-67.

Anderson, S. P. \& de Palma, A. 2004. "The Economics of Pricing Parking." Journal of Urban Economics, 55(1), pp. 1-20.

Arnott, R. \& Inci, E. (2006). "An Integrated Model of Downtown Parking and Traffic Congestion." Journal of Urban Economics, 60(3), pp. 418-442. 
Arnott, R. \& Rowse, J. (2009). "Downtown Parking in Auto City." Regional Science and Urban Economics, Volume 39, pp. 1-14.

Bomar, M. A., Becker, E. P. \& Stollof, E. R. (2009). "Urban Freight Case Studies." New York, Washington, D.C.: Federal Highway Administration.

Calthrop, E. \& Proost, S. (2006). "Regulating On-Street Parking." Regional Science and Urban Economics, 36(1), pp. 29-48.

Cao, J. \& Menendez, M. (2015). "Generalized Effects of On-Street Parking Maneuvers on the Performance of Nearby Signalized Intersections." Transportation Research Record: Journal of the Transportation Research Board, Issue 2483, pp. 30-38.

Cao, J., Menendez, M. \& Nikias, V. (2014). "The Effect of On-Street Parking on Traffic Throughput at Nearby Intersections." paper presented to the Transportation Research Board 93rd Annual Meeting, Washington, D.C., 12-16 January.

Catling, I. (1977). "A Time-Dependent Approach to Junction Delays." Traffic Engineering and Control, Volume 18, pp. 520-526.

Cheng, C., Du, Y., Sun, L. \& Ji, Y. (2016). "Review on Theoretical Delay Estimation Model for Signalized Intersections." Transport Reviews, 36(4), pp. 479-499.

Cherrett, T. et al. (2012). "Understanding Urban Freight Activity - Key Issues for Freight Planning." The Journal of Transport Geography, pp. 22-32.

City of Baltimore (2014). "Parking Authority / On-street Parking Regulations." [Online] Available at: http://archive.baltimorecity.gov/Government/QuasiAgencies/ParkingAuthority/O nstreetParkingRegulations.aspx (Accessed 16 November 2014). 
City of Chicago (2014). "Parking Restrictions for Special Types of Vehicles." [Online] Available at:

http://www.cityofchicago.org/city/en/depts/fin/supp_info/revenue/parking_your_c ommercialtruckinchicago.html (Accessed 16 November 2014).

City of New York (2014). Parking a Truck of Commercial Vehicle. [Online] Available at: http://www.nyc.gov/html/dot/html/motorist/parktruck.shtml (Accessed 16 November 2014).

City of Seattle (2014). Loadzones. [Online] Available at: http://www.seattle.gov/transportation/parking/parkingload.htm (Accessed 16 November 2014).

Daganzo, C. (1994). "The Cell Transmission Model: A Dynamic Representation of Highway Traffic Consistent with the Hydrodynamic Theory." Transportation Research Part B: Methodological, 28(4), pp. 269-287.

Daganzo, C. F. (2005). Logistics Systems Analysis. s.l. Springer Science \& Business Media.

Davis, B. A. \& Figliozzi, M. A. (2013). "A Methodology to Evaluate the Competitiveness of Electric Delivery Trucks." Transportation Research Part E, Volume 49, pp. 8-23.

Dey, S., Pérez, B., Richards, L., Pochowski, A., Sanders, M., Darst, M. \& Sanchez, E. (2019). "Demystifying Urban Curbside Freight Management: A Strategic Incremental Approach from Washington, D.C." paper presented to the Transportation Research Board 98th Annual Meeting, Washington, D.C., 13-17 January. 
Dezi, G., Dondi, G. \& Sangiorgi, C. (2010). "Urban Freight Transport in Bologna:

Planning Commercial Vehicle Loading/Unloading Zones." The Sixth

International Conference on City Logistics, Puerto Vallarta, Mexico, 30 June - 2

July.

Figliozzi, M. A. (2008). "Planning Approximations to the Average Length of Vehicle Routing Problems with Varying Customer Demands and Routing Constraints." Transportation Research Record: Journal of the Transportation Research Board, Volume 2089, pp. 1-8.

Figliozzi, M. \& Tipagornwong, C. (2017). "Impact of Last Mile Parking Availability on Commercial Vehicle Costs and Operations." In Supply Chain Forum: An International Journal, 18(2), pp. 60-68.

Fischer, M. J. \& Han, M. (2001). "NCHRP Synthesis 298: Truck Trip Generation Data: A Synthesis of Highway Practice." Washington, D.C.: Transportation Research Board.

Fosgerau, M. \& de Palma, A. (2013). "The Dynamics of Urban Traffic Congestion and the Price of Parking." Journal of Public Economics, Volume 105, pp. 106-115.

Gao, J. \& Ozbay, K. (2016). "Modeling Double Parking Impacts on Urban Street." paper presented to the Transportation Research Board 95th Annual Meeting, Washington, D.C., 10-14 January.

Garib, A., Radwan, A. E. \& Al-Deek, H. (1997). "Estimating Magnitude and Duration of Incident Delays." Journal of Transportation Engineering, 123(6), pp. 459-466.

Goolsby, M. (1971). "Influence of Incidents on Freeway Quality of Service." Highway Research Record, Volume 349. 
Gordon, R. (2007). "Parking Tickets by the Truckload / 18 S.F. Businesses Rack up Thousands of Citations, Pay City on Monthly Plan." SFGate [Online] Available at: http://www.sfgate.com/news/article/Parking-tickets-by-thetruckload-18-S-F-2615428.php (Accessed June 2016).

Gordon, R., et al. (1996). "Traffic Control Systems Handbook." Washington DC: Federal Highway Administration.

Habib, P. A. \& Crowley, K. W. (1976). "Economic Approach to Allocating Curb Space for Urban Goods Movement." Transportation Research Record: Journal of the Transportation Research Board, Volume 591, pp. 18-24.

Han, L. D., Chin, S.-M., Franzese, O. \& Hwang, H. (2005). Estimating the Impact of Pickup- and Delivery-Related Illegal Parking Activities on Traffic." Transportation Research Record: Journal of the Transportation Research Board, Volume 1906, pp. 49-55.

Hawkins, A. J. (2013). "Parking tickets: All in the Cost of Doing Business." Crain's New York Business [Online] Available at: http://www.crainsnewyork.com/article/20130526/ECONOMY/305269980/parkin g-tickets-all-in-the-cost-of-doing-business (Accessed 25 March 2014).

Holguín-Veras, J. \& Brom, M. (2008). "Trucking Costs in a Metropolitan Area: A Comparison of Alternative Estimation Approaches." paper presented to the Transportation Research Board 87th Annual Meeting, Washington, D.C., 13-17 January. 
Holguín-Veras, J. et al. (2005). "Off-peak Freight Deliveries: Challenges and Stakeholders' Perceptions." Transportation Research Record, Volume 1906, pp. $42-48$.

Hou, L. et al. (2012). "Estimation of Incident-Induced Congestion on Signalized Arteries Using Traffic Sensor Data." Tsinghua Science and Technology, 17(3), pp. 296303.

Inci, E. \& Lindsey, R. (2015). "Garage and Curbside Parking Competition with Search Congestion." Regional Science and Urban Economics, Volume 54, pp. 49-59.

Jaller, M., Holguín-Veras, J. \& Hodge, S. D. (2013). "Parking in the City: Challenges for Freight Traffic." Transportation Research Record: The Journal of the Transportation Research Board, Volume 2379, pp. 46-56.

Jung, H. Y. (1999). "Analysis of the Characteristics and Directions of Loading in the Central Commercial Area in Pusan City." The Journal of the Eastern Asia Society for Transportation Studies, September, 3(3), pp. 315-326.

Kawamura, K. (2000). "Perceived Value of Time for Truck Operators." Transportation Research Record: Journal of the Transportation Research Board, Volume 1725, pp. 31-36.

Kawamura, K. \& Lu, Y. (2007). "Evaluation of Delivery Consolidation in US Urban Areas with Logistics Cost Analysis." Transportation Research Record: Journal of the Transportation Research Board, 2008(1), pp. 34-42. 
Kawamura, K., Sriraj, P., Surat, H. \& Menninger, M. (2014). "Analysis of Factors that Affect the Frequency of Truck Parking Violations in Urban Areas." Transportation Research Record: Journal of the Transportation Research Board, Volume 2411, pp. 20-26.

Keegan, A. \& Gonzales, E. (2016). "Evaluating Capacity and Delay for Signalized Arterials with Freight Deliveries." Transportation Research Procedia, Volume 15, pp. 161-175.

Kimber, R. M. \& Hollis, E. M. (1979). "TRRL Laboratory Report 909: Traffic Queues and Delays at Road Junctions." Crowthorne, United Kingdom, Transport and Road Research Laboratory.

Kladeftiras, M. \& Antoniou, C. (2013). "Simulation-based Assessment of DoubleParking Impacts on Traffic and Environmental Conditions." Transportation Research Record: Journal of the Transportation Research Board, Volume 2390, pp. 121-130.

Kobus, M., Gutiérrez-i-Puigarnau, E., Rietveld, P. \& Van Ommeren, J. N. (2013). "The Cn-Street Parking Premium and Car Drivers' Choice Between Street and Garage Parking." Regional Science and Urban Economics, 43(2), pp. 395-403.

Lam, W., Li, Z.-C., Huang, H.-J. \& Wong, S. C. (2006). "Modeling Time-Dependent Travel Choice Problems in Road Networks with Multiple User Classes and Multiple Parking Facilities." Transportation Research Part B: Methodological, 40(5), pp. 368-395. 
Langevin, A., Mbaraga, P. \& Cambell, J. F. (1996). "Continuous Approximation Models in Freight Distribution: An Overview". Transportation Research Part B: Methodological, 30(3), pp. 163-188.

Li, J., Lan, C. J. \& Gu, X. (2006). "Estimation of Incident Delay and Its Uncertainty on Freeway Networks." Transportation Research Record: Journal of the Transportation Research Board, Volume 1959, pp. 37-45.

Long, J., Gao, Z., Orenstein, P. \& Ren, H. (2012). "Control Strategies for Dispersing Incident-Based Traffic Jams in Two-Way Grid Networks." IEEE Transactions on Intelligent Transportation Systems, 13(2), pp. 469-481.

Lopez, C., Gonzalez-Feliu, J., Chiabaut, N. \& Leclercq, L. (2016). "Assessing the Impacts of Goods Deliveries' Double Line Parking on the Overall Traffic Under Realistic Conditions." Bordeaux, France, IGI Global.

Lu, B. \& Viegas, J. (2007). "The Analysis of the Influences of the Double Parking Vehicles to the General Traffic Flow." Chengdu, China, American Society of Civil Engineers, pp. 3121-3126.

Maes, J. \& Vanelslander, T. (2012). "The Use of Bicycle Messengers in the Logistics Chain, Concepts Further Revised." Mallorca, Elsevier, pp. 409-423.

Massahi, A. \& Cutillo, M. (2016). "Estimating the Capacity Impacts of Urban Street Incidents." paper presented to the Transportation Research Board 95th Annual Meeting, Washington, D.C., 10-14 January.

McLeod, F. \& Cherrett, T. (2011). "Loading Bay Booking and Control for Urban Freight." International Journal of Logistics Research and Applications: A Leading Journal of Supply Chain Management, pp. 385-397. 
Mei, Q., Hussein, M. I. \& Horowitz, A. J. (2013). "Establishing Values of Time for Freight Trucks for Better Understanding of Impact of Toll Policies."

Transportation Research Record: Journal of the Transportation Research Board, Volume 2344, pp. 135-143.

Menendez, M. \& Daganzo, C. (2004). "Assessment of the Impact of Incidents Near Bottlenecks: Strategies to Reduce Delay." Transportation Research Record: Journal of the Transportation Research Board, Volume 1867, pp. 53-59.

Messer, C., Dudek, C. \& Friebele, J.(1973). "Method for Predicting Travel Time and Other Operational Measures in Real-Time During Freeway Incident Conditions." Highway Research Record, Volume 461, pp. 1-16.

Miao, Q., Wang, X. B. \& Adams, T. M. (2014). "Assessing the Value of Delay to ShortHaul Carriers." Journal of Applied Research and Technology, 12(4), pp. 642-653.

Millard-Ball, A., Weinberger, R. R. \& Hampshire, R. C. (2014). "Is the Curb 80\% Full or $20 \%$ Empty? Assessing the Impacts of San Francisco's Parking Pricing Experiment." Transportation Research Part A: Policy and Practice, Volume 63, pp. 76-92.

Morris, A. G., Kornhauser, A. L. \& Kay, M. J. (1999). "Getting the Goods Delivered in Dense Urban Areas: A Snapshot of Last Link of the Supply Chain." Transportation Research Record: The Journal of the Transportation Research Board, Volume 1653, pp. 34-41.

Muñuzuri, J., Cortés, P., Guadix, J. \& Onieva, L. (2012). "City Logistics in Spain: Why it Might Never Work." Cities, Volume 29, pp. 133-141. 
Muñuzuri, J., Cuberos, M., Abaurrea, F. \& Escudero, A. (2017). "Improving the Design of Urban Loading Zone Systems". Journal of Transport Geography, Volume 59, pp. $1-13$.

Murphy, Jr., P. R. \& Wood, D. F. (2010). "Contemporary Logistics." New Jersey: Prentice Hall.

Nourinejad, M. \& Roorda, M. J. (2017). "Parking enforcement policies for commercial vehicles." Transportation Research Part A: Policy and Practice, Volume 102, pp. $33-50$.

Oregon Department of Transportation (2014). "The Value of Travel-Time: Estimates of the Hourly Value of Time for Vehicles in Oregon 2013." [Online] Available at: http://library.state.or.us/repository/2009/200902121450374/2013.pdf (Accessed 15 July 2017).

Oregon. Gov. (2012). "Service Vehicle Parking Policy." [Online] Available at: http://www.oregon.gov/DAS/EAM/FPS/docs/service_appl_policy_map.pdf (Accessed 16 November 2014).

Pivo, G., Carlson, D., Kitchen, M. \& Billen, D. (1997). "Learning from Trucks: Moving Goods in Compact, Livable Urban Areas." Seattle: Washington State Transportation Center.

Portilla, A. I., Oreña, B. A., Berodia, J. L. \& Díaz, F. J. (2009). "Using M/M/ø Queueing Model in On-Street Parking Maneuvers." Journal of Transportation Engineering, 135(8), pp. 527-535. 
Quadrifoglio, L. \& Li, X. (2009). "A Methodology to Derive the Critical Demand Density for Designing and Operating Feeder Transit Services." Transportation Research Part B: Methodological, 43(10), pp. 922-935.

Rosenfield, A., Lamers, J., Nourinejad, M. \& Roorda, M. J. (2016). "Investigation of Commercial Vehicle Parking Permits in Toronto, Ontario, Canada." Transportation Research Record: Journal of the Transportation Research Board, Volume 2547, pp. 11-18.

Shoup, D. C. (2006). "Cruising for Parking." Transport Policy, Issue 13, pp. 476-486.

Shu-chun, W., Jun, C. \& Hui, Z. (2008). "The Optimization Model of Setting the Curb Parking Facilities." Taipei, Institute of Electrical and Electronics Engineers.

Simon, T. \& Conway, A. (2019). "Before/After Freight Impact Analysis of the New York City Clear Curbs Initiative." paper presented to the Transportation Research Board 98th Annual Meeting, Washington, D.C., 13-17 January.

Smith, B., Qin, L. \& Venkatanarayana, R. (2003). "Characterization of Freeway Capacity Reduction Resulting from Traffic Accidents." Journal of Transportation Engineering, 129(4), pp. 362-368.

Soumya, D., Benito, P., Richards, L., Pochowski, A., Sanders, M., Darst, M. \& Sanchez, E. (2019). "Demystifying Urban Curbside Freight Management: A Strategic Incremental Approach from Washington, D.C." paper presented to the Transportation Research Board 98th Annual Meeting, Washington, D.C., 13-17 January.

Sullivan, E. C. (1997). "New Model for Predicting Freeway Incidents and Incident Delays." Journal of Transportation Engineering, 123(4), pp. 267-275. 
The Transportation Research Board (1971). "Parking Principle: Highway Research Board Special Report 125." Washington, D.C.: The Transportation Research Board. Tipagornwong, C. \& Figliozzi, M. (2014). "An Analysis of the Competitiveness of Freight Tricycle Delivery Services in Urban Areas." Transportation Research Record: Journal of the Transportation Research Board, Volume 2410, pp. 76-84.

Tozzi, M., Corazza, M. V. \& Musso, A. (2012). "Trip Chain Behavior of Commercial Vehicles in Urban Areas: The Parma Case Study." Padova, Italy, Elsevier, pp. 113.

Waters, W. G., Wong, C. \& Megale, K. (1995). "The Value of Commercial Vehicle Time Savings for the Evaluation of Highway Investments: A Resource Saving Approach." Journal of the Transportation Research Forum, 35(1), pp. 97-113.

Wenneman, A., Habib, K. M. N. \& Roorda, M. J. (2015). "Disaggregate Analysis of Relationships Between Commercial Vehicle Parking Citations, Parking Supply, and Parking Demand." Transportation Research Record: Journal of the Transportation Research Board, Volume 2478, pp. 28-34.

Wirasinghe, S. C. (1978). "Determination of Traffic Delays From Shock-Wave Analysis." Transportation Research, 12(5), pp. 343-348.

Wong, W. T., Wong, S. C. \& Tong, C. O. (2003). "Sheared Delay Formulae for the TRANSYT Traffic Model: A Review and Calibration." Transport Reviews, 23(1), pp. 1-20.

Wu, X. \& Liu, H. X. (2011). "A Shockwave Profile Model for Traffic Flow on Congested Urban Arterials." Transportation Research Part B: Methodological, 45(10), pp. $1768-1786$. 
Zheng, N. \& Geroliminis, N. (2016). "Modeling and Optimization of Multimodal Urban Networks with Limited Parking and Dynamic Pricing." Transportation Research Part B: Methodological, Volume 83, pp. 36-58.

Zhou, J. (2014). "An Integrated Model of Parking Pricing and Cruising." Changsha, China, American Society of Civil Engineers. 
Appendix A: Full derivation of parking supply allocation and pricing models

Derivation for solutions and the optimality condition test for all models

Revenue models of 4 scenarios (in Chapter 5)

Social optimum models for 4 scenarios (in Chapter 7) 


\section{$\underline{\text { Revenue model (scenario 1): formulation, derivation }}$}

\section{Scenario information}

$\lambda_{c}^{*} \geq \lambda_{c}$

All commercial vehicles parking on street

$\lambda_{p}^{*} \geq \lambda_{p}$

All passenger cars parking on street

Revenue and cost of commercial vehicles parking

$\underline{\text { Per-stop monetary cost of a commercial vehicle parking on street }}$

$\frac{c_{c o}}{\mu_{c}}$

$\underline{\text { Per-stop waiting time of a commercial vehicle parking on street }}$

$\alpha_{w c}\left(\frac{\lambda_{c}}{n_{c} \mu_{c}}\right)^{\gamma_{c}} \quad \gamma_{c} \geq 1$

$\underline{\text { Per-stop parking delay cost of a commercial vehicle parking on street }}$

$c_{c t} \alpha_{w c}\left(\frac{\lambda_{c}}{n_{c} \mu_{c}}\right)^{\gamma_{c}}$

$\underline{\text { Per-stop double parking cost of a commercial vehicle }}$

$\frac{c_{v} n_{e}}{\mu_{c} \tau_{e}}$

$\underline{\text { Relationship between on-street parking and double parking }}$

$$
\begin{aligned}
& \frac{c_{c o}}{\mu_{c}}+c_{c t} \alpha_{w c}\left(\frac{\lambda_{c}}{n_{c} \mu_{c}}\right)^{\gamma_{c}} \leq \frac{c_{v} n_{e}}{\mu_{c} \tau_{e}} \\
& c_{c o} \leq \frac{c_{v} n_{e}}{\tau_{e}}-c_{c t} \alpha_{w c} \mu_{c}\left(\frac{\lambda_{c}}{n_{c} \mu_{c}}\right)^{\gamma_{c}} \\
& n_{c} \geq\left(\frac{\lambda_{c}}{\mu_{c}}\right)\left(\frac{c_{c t} \alpha_{w c} \mu_{c}}{\frac{c_{v} n_{e}}{\tau_{e}}-c_{c o}}\right)^{\frac{1}{\gamma_{c}}}
\end{aligned}
$$




\section{Revenue and cost of passenger cars parking}

Per-stop monetary cost of a passenger car parking on street

$\frac{c_{p o}}{\mu_{p}}$

$\underline{\text { Per-stop parking waiting time of a passenger car }}$

$\alpha_{w p}\left(\frac{\lambda_{p}}{n_{p} \mu_{p}}\right)^{\gamma_{p}} \quad \gamma_{p} \geq 1$

$\underline{\text { Per-stop parking delay cost of a passenger car }}$

$c_{p t}(1+\vartheta) \alpha_{w p}\left(\frac{\lambda_{p}}{n_{p} \mu_{p}}\right)^{\gamma_{p}}$

$\underline{\text { Per-stop parking cost of a passenger car parking off street }}$

$\frac{c_{p f}}{\mu_{p}}+c_{p f f}$

Relationship between on-street parking and double parking

$$
\begin{aligned}
& \frac{c_{p o}}{\mu_{p}}+c_{p t}(1+\vartheta) \alpha_{w p}\left(\frac{\lambda_{p}}{n_{p} \mu_{p}}\right)^{\gamma_{p}} \leq \frac{c_{p f}}{\mu_{p}}+c_{p f f} \\
& c_{p o} \leq c_{p f}+c_{p f f} \mu_{p}-c_{p t}(1+\vartheta) \alpha_{w p} \mu_{p}\left(\frac{\lambda_{p}}{n_{p} \mu_{p}}\right)^{\gamma_{p}} \\
& n_{p} \geq\left(\frac{\lambda_{p}}{\mu_{p}}\right)\left[\frac{c_{p t}(1+\vartheta) \alpha_{w p} \mu_{p}}{c_{p f}+c_{p f f} \mu_{p}-c_{p o}}\right]^{\frac{1}{\gamma_{p}}}
\end{aligned}
$$


Summary of the relevant revenue and cost (unit: dollars per hour)

\begin{tabular}{|l|c|}
\hline Components & Formula \\
\hline Commercial parking delay cost & 0 \\
\hline Commercial maneuver traffic delay cost & 0 \\
\hline Double parking delay cost & 0 \\
\hline Passenger parking delay cost & 0 \\
\hline Passenger maneuver traffic delay cost & $\frac{c_{c o} \lambda_{c}}{\mu_{c}}$ \\
\hline On-street commercial vehicle parking revenue & $\frac{c_{p o} \lambda_{p}}{\mu_{p}}$ \\
\hline On-street passenger vehicle parking revenue & \\
\hline
\end{tabular}

\section{Objective function}

$y_{\text {rev }}=\frac{c_{c o} \lambda_{c}}{\mu_{c}}+\frac{c_{p o} \lambda_{p}}{\mu_{p}}$

\section{$\underline{\text { Constraint }}$}

Meter rate

$$
\begin{aligned}
& c_{c o} \leq \frac{c_{v} n_{e}}{\tau_{e}}-c_{c t} \alpha_{w c} \mu_{c}\left(\frac{\lambda_{c}}{n_{c} \mu_{c}}\right)^{\gamma_{c}} \\
& c_{p o} \leq c_{p f}+c_{p f f} \mu_{p}-c_{p t}(1+\vartheta) \alpha_{w p} \mu_{p}\left(\frac{\lambda_{p}}{n_{p} \mu_{p}}\right)^{\gamma_{p}}
\end{aligned}
$$

Available parking length

$$
\begin{aligned}
& n_{c} l_{c}+n_{p} l_{p}=l_{b} \\
& n_{c} \geq\left(\frac{\lambda_{c}}{\mu_{c}}\right)\left(\frac{c_{c t} \alpha_{w c} \mu_{c}}{\frac{c_{v} n_{e}}{\tau_{e}}-c_{c o}}\right)^{\frac{1}{\gamma_{c}}}
\end{aligned}
$$


$n_{p} \geq\left(\frac{\lambda_{p}}{\mu_{p}}\right)\left[\frac{c_{p t}(1+\vartheta) \alpha_{w p} \mu_{p}}{c_{p f}+c_{p f f} \mu_{p}-c_{p o}}\right]^{\frac{1}{\gamma_{p}}}$

Since $n_{c} l_{c}+n_{p} l_{p}=l_{b}, \quad n_{c}=\frac{l_{b}-n_{p} l_{p}}{l_{c}}$

The constraint on $n_{c}$ become

$n_{p} \leq \frac{l_{b}}{l_{p}}-\frac{l_{c}}{l_{p}}\left(\frac{\lambda_{c}}{\mu_{c}}\right)\left(\frac{c_{c t} \alpha_{w c} \mu_{c}}{\frac{c_{v} n_{e}}{\tau_{e}}-c_{c o}}\right)^{\frac{1}{\gamma_{c}}}$

\section{Optimal $c_{\text {po }}$}

\section{Derivation of solution}

The revenue is a linear function of $c_{p o}$. The constraint on $c_{p o}$ is,

$c_{p o} \leq c_{p f}+c_{p f f} \mu_{p}-c_{p t}(1+\vartheta) \alpha_{w p} \mu_{p}\left(\frac{\lambda_{p}}{n_{p} \mu_{p}}\right)^{\gamma_{p}}$

Since the problem is a linear programming, the optimal $c_{p o}$ is one of extreme points of the feasible region,

$c_{p o}^{*} \in\left\{0, c_{p f}+c_{p f f} \mu_{p}-c_{p t}(1+\vartheta) \alpha_{w p} \mu_{p}\left(\frac{\lambda_{p}}{n_{p} \mu_{p}}\right)^{\gamma_{p}}\right\}$

The revenue is an increasing function of $c_{p o}$. Therefore, the optimal solution is,

$c_{p o}^{*}=c_{p f}+c_{p f f} \mu_{p}-c_{p t}(1+\vartheta) \alpha_{w p} \mu_{p}\left(\frac{\lambda_{p}}{n_{p} \mu_{p}}\right)^{\gamma_{p}}$ 


\section{Optimal $c_{c o}$}

\section{Derivation of solution}

The revenue is a linear function of $c_{c o}$. The constraint on $c_{c o}$ is,

$c_{c o} \leq \frac{c_{v} n_{e}}{\tau_{e}}-c_{c t} \alpha_{w c} \mu_{c}\left(\frac{\lambda_{c}}{n_{c} \mu_{c}}\right)^{\gamma_{c}}$

Since the problem is a linear programming, the optimal $c_{c o}$ is one of extreme points of the feasible region,

$c_{c o}^{*} \in\left\{0, \frac{c_{v} n_{e}}{\tau_{e}}-c_{c t} \alpha_{w c} \mu_{c}\left(\frac{\lambda_{c}}{n_{c} \mu_{c}}\right)^{\gamma_{c}}\right\}$

The revenue is an increasing function of $c_{c o}$. Therefore, the optimal solution is,

$c_{c o}^{*}=\frac{c_{v} n_{e}}{\tau_{e}}-c_{c t} \alpha_{w c} \mu_{c}\left(\frac{\lambda_{c}}{n_{c} \mu_{c}}\right)^{\gamma_{c}}$

\section{Optimal $n_{p}$}

\section{Derivation of solution}

The constraint on $n_{p}$ is,

$$
\begin{aligned}
& n_{p} \geq\left(\frac{\lambda_{p}}{\mu_{p}}\right)\left[\frac{c_{p t}(1+\vartheta) \alpha_{w p} \mu_{p}}{c_{p f}+c_{p f f} \mu_{p}-c_{p o}}\right]^{\frac{1}{\gamma_{p}}} \\
& n_{p} \leq \frac{l_{b}}{l_{p}}-\frac{l_{c}}{l_{p}}\left(\frac{\lambda_{c}}{\mu_{c}}\right)\left(\frac{c_{c t} \alpha_{w c} \mu_{c}}{\frac{c_{v} n_{e}}{\tau_{e}}-c_{c o}}\right)^{\frac{1}{\gamma_{c}}}
\end{aligned}
$$

The extreme points of the feasible region are,

$$
n_{p}^{*} \in\left\{\left(\frac{\lambda_{p}}{\mu_{p}}\right)\left[\frac{c_{p t}(1+\vartheta) \alpha_{w p} \mu_{p}}{c_{p f}+c_{p f f} \mu_{p}-c_{p o}}\right]^{\frac{1}{\gamma_{p}}}, \frac{l_{b}}{l_{p}}-\frac{l_{c}}{l_{p}}\left(\frac{\lambda_{c}}{\mu_{c}}\right)\left(\frac{c_{c t} \alpha_{w c} \mu_{c}}{\frac{c_{v} n_{e}}{\tau_{e}}-c_{c o}}\right)^{\frac{1}{\gamma_{c}}}\right\}
$$




\section{$\underline{\text { Revenue model (scenario 2): formulation, derivation }}$}

\section{Scenario information}

$\lambda_{c}^{*} \geq \lambda_{c}$

All commercial vehicles parking on street

$\lambda_{p}^{*}<\lambda_{p}$

Some passenger cars parking off street

Revenue and cost of commercial vehicles parking

$\underline{\text { Per-stop monetary cost of a commercial vehicle parking on street }}$

$\frac{c_{c o}}{\mu_{c}}$

$\underline{\text { Per-stop waiting time of a commercial vehicle parking on street }}$

$\alpha_{w c}\left(\frac{\lambda_{c}}{n_{c} \mu_{c}}\right)^{\gamma_{c}} \quad \gamma_{c} \geq 1$

$\underline{\text { Per-stop parking delay cost of a commercial vehicle parking on street }}$

$c_{c t} \alpha_{w c}\left(\frac{\lambda_{c}}{n_{c} \mu_{c}}\right)^{\gamma_{c}}$

$\underline{\text { Per-stop double parking cost of a commercial vehicle }}$

$\frac{c_{v} n_{e}}{\mu_{c} \tau_{e}}$

$\underline{\text { Relationship between on-street parking and double parking }}$

$$
\begin{aligned}
& \frac{c_{c o}}{\mu_{c}}+c_{c t} \alpha_{w c}\left(\frac{\lambda_{c}}{n_{c} \mu_{c}}\right)^{\gamma_{c}} \leq \frac{c_{v} n_{e}}{\mu_{c} \tau_{e}} \\
& c_{c o} \leq \frac{c_{v} n_{e}}{\tau_{e}}-c_{c t} \alpha_{w c} \mu_{c}\left(\frac{\lambda_{c}}{n_{c} \mu_{c}}\right)^{\gamma_{c}} \\
& n_{c} \geq\left(\frac{\lambda_{c}}{\mu_{c}}\right)\left(\frac{c_{c t} \alpha_{w c} \mu_{c}}{\frac{c_{v} n_{e}}{\tau_{e}}-c_{c o}}\right)^{\frac{1}{\gamma_{c}}}
\end{aligned}
$$




\section{Revenue and cost of passenger cars parking}

$\underline{\text { Per-stop monetary cost of a passenger car parking on street }}$

$\frac{c_{p o}}{\mu_{p}}$

Per-stop parking waiting time of a passenger car

$\alpha_{w p}\left(\frac{\lambda_{p}^{*}}{n_{p} \mu_{p}}\right)^{\gamma_{p}} \quad \gamma_{p} \geq \gamma_{c} \geq 1$

$\underline{\text { Per-stop parking delay cost of a passenger car }}$

$c_{p t}(1+\vartheta) \alpha_{w p}\left(\frac{\lambda_{p}^{*}}{n_{p} \mu_{p}}\right)^{\gamma_{p}}$

$\underline{\text { Per-stop parking cost of a passenger car parking off street }}$

$\frac{c_{p f}}{\mu_{p}}+c_{p f f}$

Breakeven demand for "paying on-street" passenger parking: $\lambda_{p}^{*}$

$$
\begin{aligned}
& \frac{c_{p o}}{\mu_{p}}+c_{p t}(1+\vartheta) \alpha_{w p}\left(\frac{\lambda_{p}^{*}}{n_{p} \mu_{p}}\right)^{\gamma_{p}}=\frac{c_{p f}}{\mu_{p}}+c_{p f f} \\
& c_{p t}(1+\vartheta) \alpha_{w p}\left(\frac{\lambda_{p}^{*}}{n_{p} \mu_{p}}\right)^{\gamma_{p}}=\frac{c_{p f}}{\mu_{p}}+c_{p f f}-\frac{c_{p o}}{\mu_{p}} \\
& \left(\frac{\lambda_{p}^{*}}{n_{p} \mu_{p}}\right)^{\gamma_{p}}=\frac{c_{p f}+c_{p f f} \mu_{p}-c_{p o}}{c_{p t}(1+\vartheta) \alpha_{w p} \mu_{p}} \\
& \lambda_{p}^{*}=n_{p} \mu_{p}\left[\frac{c_{p f}+c_{p f f} \mu_{p}-c_{p o}}{c_{p t}(1+\vartheta) \alpha_{w p} \mu_{p}}\right]^{\frac{1}{\gamma_{p}}}
\end{aligned}
$$


Summary of the relevant revenue and cost (unit: dollars per hour)

\begin{tabular}{|l|c|}
\hline Components & Formula \\
\hline Commercial parking delay cost & 0 \\
\hline Commercial maneuver traffic delay cost & 0 \\
\hline Double parking delay cost & 0 \\
\hline Passenger parking delay cost & 0 \\
\hline Passenger maneuver traffic delay cost & $\frac{c_{c o} \lambda_{c}}{\mu_{c}}$ \\
\hline On-street commercial vehicle parking revenue & $\frac{c_{p o} \lambda_{p}^{*}}{\mu_{p}}$ \\
\hline On-street passenger vehicle parking revenue & \\
\hline
\end{tabular}

\section{Objective function}

$y_{r e v}=\frac{c_{c o} \lambda_{c}}{\mu_{c}}+\frac{c_{p o} \lambda_{p}^{*}}{\mu_{p}}$

From equation (B.3), the objective function becomes,

$y_{r e v}=\frac{c_{c o} \lambda_{c}}{\mu_{c}}+n_{p} c_{p o}\left[\frac{c_{p f}+c_{p f f} \mu_{p}-c_{p o}}{c_{p t}(1+\vartheta) \alpha_{w p} \mu_{p}}\right]^{\frac{1}{\gamma_{p}}}$

\section{$\underline{\text { Constraint }}$}

Meter rate

$c_{c o} \leq \frac{c_{v} n_{e}}{\tau_{e}}-c_{c t} \alpha_{w c} \mu_{c}\left(\frac{\lambda_{c}}{n_{c} \mu_{c}}\right)^{\gamma_{c}}$

Available parking length

$n_{c} l_{c}+n_{p} l_{p}=l_{b}$

$n_{c} \geq\left(\frac{\lambda_{c}}{\mu_{c}}\right)\left(\frac{c_{c t} \alpha_{w c} \mu_{c}}{\frac{c_{v} n_{e}}{\tau_{e}}-c_{c o}}\right)^{\frac{1}{\gamma_{c}}}$ 
$n_{p} \geq 0$

Since $n_{c} l_{c}+n_{p} l_{p}=l_{b}, \quad n_{c}=\frac{l_{b}-n_{p} l_{p}}{l_{c}}$

The constraints become

$c_{c o} \leq \frac{c_{v} n_{e}}{\tau_{e}}-c_{c t} \alpha_{w c} \mu_{c}\left(\frac{\lambda_{c}}{n_{c} \mu_{c}}\right)^{\gamma_{c}}$

$n_{p} \leq \frac{l_{b}}{l_{p}}-\frac{l_{c}}{l_{p}}\left(\frac{\lambda_{c}}{\mu_{c}}\right)\left(\frac{c_{c t} \alpha_{w c} \mu_{c}}{\frac{c_{v} n_{e}}{\tau_{e}}-c_{c o}}\right)^{\frac{1}{\gamma_{c}}}$

$n_{p} \geq 0$

\section{Optimal $c_{p o}$}

\section{Derivation of solution}

From equation (A.5)

Assume $c_{c o}$ and $n_{p}$ constant. Therefore, all the constraints are not necessary. The revenue maximization problem becomes,

$y_{\text {rev }}=\frac{c_{c o} \lambda_{c}}{\mu_{c}}+n_{p} c_{p o}\left[\frac{c_{p f}+c_{p f f} \mu_{p}-c_{p o}}{c_{p t}(1+\vartheta) \alpha_{w p} \mu_{p}}\right]^{\frac{1}{\gamma_{p}}}$

$\frac{\partial y}{\partial c_{p o}}=\frac{n_{p}\left[\gamma_{p} c_{p f}+\gamma_{p} c_{p f f} \mu_{p}-\left(\gamma_{p}+1\right) c_{p o}\right]}{\gamma_{p}\left[c_{p t}(1+\vartheta) \alpha_{w p} \mu_{p}\right]^{\frac{1}{\gamma_{p}}}\left[c_{p f}+c_{p f f} \mu_{p}-c_{p o}\right]^{\frac{\gamma_{p}-1}{\gamma_{p}}}}=0$

Optimal solution

$c_{p o}^{*}=\frac{\gamma_{p}\left(c_{p f}+c_{p f f} \mu_{p}\right)}{\left(\gamma_{p}+1\right)}$

Check the optimality condition

$$
\frac{\partial^{2} L}{\partial c_{p o}^{2}}=-\frac{\left(l_{b}-n_{c} l_{c}\right)\left(c_{p f}+c_{p f f} \mu_{p}\right)}{\gamma_{p} l_{p}\left[c_{p t}(1+\vartheta) \alpha_{w p} \mu_{p}\right]^{\frac{1}{\gamma_{p}}}}\left(\frac{\gamma_{p}+1}{c_{p f}+c_{p f f} \mu_{p}}\right)^{\frac{2 \gamma_{p}-1}{\gamma_{p}}}<0
$$

Therefore, the solution is a local maximum. 


\section{Optimal $c_{c o}$}

\section{Derivation of solution}

The revenue is a linear function of $c_{c o}$. The constraint on $c_{c o}$ is,

$c_{c o} \leq \frac{c_{v} n_{e}}{\tau_{e}}-c_{c t} \alpha_{w c} \mu_{c}\left(\frac{\lambda_{c}}{n_{c} \mu_{c}}\right)^{\gamma_{c}}$

Since the problem is a linear programming, the optimal $c_{c o}$ is one of extreme points of the feasible region,

$c_{c o}^{*} \in\left\{0, \frac{c_{v} n_{e}}{\tau_{e}}-c_{c t} \alpha_{w c} \mu_{c}\left(\frac{\lambda_{c}}{n_{c} \mu_{c}}\right)^{\gamma_{c}}\right\}$

The revenue is an increasing function of $c_{c o}$. Therefore, the optimal solution is,

$c_{c o}^{*}=\frac{c_{v} n_{e}}{\tau_{e}}-c_{c t} \alpha_{w c} \mu_{c}\left(\frac{\lambda_{c}}{n_{c} \mu_{c}}\right)^{\gamma_{c}}$

\section{Optimal $n_{p}$}

\section{Derivation of solution}

The revenue is a linear function of $n_{p}$. The constraint on $n_{p}$ is,

$n_{p} \leq \frac{l_{b}}{l_{p}}-\frac{l_{c}}{l_{p}}\left(\frac{\lambda_{c}}{\mu_{c}}\right)\left(\frac{c_{c t} \alpha_{w c} \mu_{c}}{\frac{c_{v} n_{e}}{\tau_{e}}-c_{c o}}\right)^{\frac{1}{\gamma_{c}}}$

Since the problem is a linear programming, the optimal $n_{p}$ is one of the extreme points of the feasible region. The revenue is an increasing function of $n_{p}$. Therefore, the optimal solution is,

$n_{p}^{*}=\frac{l_{b}}{l_{p}}-\frac{l_{c}}{l_{p}}\left(\frac{\lambda_{c}}{\mu_{c}}\right)\left(\frac{c_{c t} \alpha_{w c} \mu_{c}}{\frac{c_{v} n_{e}}{\tau_{e}}-c_{c o}}\right)^{\frac{1}{\gamma_{c}}}$ 


\section{$\underline{\text { Revenue model (scenario 3): formulation, derivation }}$}

\section{Scenario information}

$\lambda_{c}^{*}<\lambda_{c}$

Some commercial vehicles double parking

$\lambda_{p}^{*} \geq \lambda_{p}$

All the passenger cars parking on street

\section{Cost of commercial vehicles parking}

$\underline{\text { Per-stop monetary cost of commercial vehicles parking on street }}$

$\frac{c_{c o}}{\mu_{c}}$

Per-stop waiting time of commercial vehicles parking on street

$\alpha_{w c}\left(\frac{\lambda_{c}^{*}}{n_{c} \mu_{c}}\right)^{\gamma_{c}} \quad \gamma_{c} \geq 1$

Per-stop parking search cost of commercial vehicles parking on street

Assumption: The time constraint is binding.

$c_{c t} \alpha_{w c}\left(\frac{\lambda_{c}^{*}}{n_{c} \mu_{c}}\right)^{\gamma_{c}}$

Per-stop double parking cost of commercial vehicles

$\frac{c_{v} n_{e}}{\mu_{c} \tau_{e}}$

Breakeven demand for "paying on-street" commercial parking: $\lambda_{c}^{*}$

(no impact of passenger cars on commercial vehicles)

$\frac{c_{c o}}{\mu_{c}}+c_{c t} \alpha_{w c}\left(\frac{\lambda_{c}^{*}}{n_{c} \mu_{c}}\right)^{\gamma_{c}}=\frac{c_{v} n_{e}}{\mu_{c} \tau_{e}}$

$\left(\frac{\lambda_{c}^{*}}{n_{c} \mu_{c}}\right)^{\gamma_{c}}=\frac{c_{v} n_{e}-c_{c o} \tau_{e}}{c_{c t} \alpha_{w c} \mu_{c} \tau_{e}}$

$\lambda_{c}^{*}=n_{c} \mu_{c}\left(\frac{c_{v} n_{e}-c_{c o} \tau_{e}}{c_{c t} \alpha_{w c} \mu_{c} \tau_{e}}\right)^{\frac{1}{\gamma_{c}}}$ 


\section{Cost of passenger cars parking}

Per-stop monetary cost of a passenger car parking on street

$\frac{c_{p o}}{\mu_{p}}$

Per-stop parking waiting time of a passenger car

$\alpha_{w p}\left(\frac{\lambda_{p}}{n_{p} \mu_{p}}\right)^{\gamma_{p}} \quad \gamma_{p} \geq \gamma_{c} \geq 1$

Per-stop parking search cost of a passenger car

Assumption: The time constraint is binding.

$c_{p t}(1+\vartheta) \alpha_{w p}\left(\frac{\lambda_{p}}{n_{p} \mu_{p}}\right)^{\gamma_{p}}$

Expected number of double-parking events that a passenger car will find while searching

$t_{s}\left(\lambda_{c}-\lambda_{c}^{*}\right)$

$\underline{\text { Per-stop delay cost of a passenger car in queue caused by double-parking commercial }}$

vehicles

$c_{p t} f_{d b} t_{s}\left(\lambda_{c}-\lambda_{c}^{*}\right)$

Per-stop parking cost of a passenger car parking off street

$\frac{c_{p f}}{\mu_{p}}+c_{p f f}$

$\underline{\text { Relationship between on-street parking and double parking }}$

$$
\begin{aligned}
& \frac{c_{p o}}{\mu_{p}}+c_{p t}(1+\vartheta) \alpha_{w p}\left(\frac{\lambda_{p}}{n_{p} \mu_{p}}\right)^{\gamma_{p}}+c_{p t} f_{d b} t_{s}\left(\lambda_{c}-\lambda_{c}^{*}\right) \leq \frac{c_{p f}}{\mu_{p}}+c_{p f f} \\
& c_{p o} \leq c_{p f}+c_{p f f} \mu_{p}-c_{p t}(1+\vartheta) \alpha_{w p} \mu_{p}\left(\frac{\lambda_{p}}{n_{p} \mu_{p}}\right)^{\gamma_{p}}-c_{p t} \mu_{p} f_{d b} t_{s}\left(\lambda_{c}-\lambda_{c}^{*}\right) \\
& n_{p} \geq\left(\frac{\lambda_{p}}{\mu_{p}}\right)\left[\frac{c_{p t}(1+\vartheta) \alpha_{w p} \mu_{p}}{c_{p f}+c_{p f f} \mu_{p}-c_{p o}-c_{p t} \mu_{p} f_{d b} t_{s}\left(\lambda_{c}-\lambda_{c}^{*}\right)}\right]^{\frac{1}{\gamma_{p}}}
\end{aligned}
$$


Summary of the relevant revenue and cost (unit: dollars per hour)

\begin{tabular}{|l|c|}
\hline Components & Formula \\
\hline Commercial parking delay cost & 0 \\
\hline Commercial maneuver traffic delay cost & 0 \\
\hline Double parking delay cost & 0 \\
\hline Passenger parking delay cost & 0 \\
\hline Passenger maneuver traffic delay cost & $\frac{c_{c o} \lambda_{c}^{*}}{\mu_{c}}$ \\
\hline On-street commercial vehicle parking revenue & $\frac{c_{p o} \lambda_{p}}{\mu_{p}}$ \\
\hline On-street passenger vehicle parking revenue & \\
\hline
\end{tabular}

\section{Objective function}

$y_{r e v}=\frac{c_{c o} \lambda_{c}^{*}}{\mu_{c}}+\frac{c_{p o} \lambda_{p}}{\mu_{p}}$

From equations (E.1), (E.2) and (E.3), the objective function becomes,

$y_{r e v}=n_{c} c_{c o}\left(\frac{c_{v} n_{e}-c_{c o} \tau_{e}}{c_{c t} \alpha_{w c} \mu_{c} \tau_{e}}\right)^{\frac{1}{\gamma_{c}}}+\frac{c_{p o} \lambda_{p}}{\mu_{p}}$

\section{$\underline{\text { Constraint }}$}

Meter rate

$c_{p o} \leq c_{p f}+c_{p f f} \mu_{p}-c_{p t}(1+\vartheta) \alpha_{w p} \mu_{p}\left(\frac{\lambda_{p}}{n_{p} \mu_{p}}\right)^{\gamma_{p}}-c_{p t} \mu_{p} f_{d b} t_{s}\left(\lambda_{c}-\lambda_{c}^{*}\right)$

Available parking length

$n_{p} \geq\left(\frac{\lambda_{p}}{\mu_{p}}\right)\left[\frac{c_{p t}(1+\vartheta) \alpha_{w p} \mu_{p}}{c_{p f}+c_{p f f} \mu_{p}-c_{p o}-c_{p t} \mu_{p} f_{d b} t_{s}\left(\lambda_{c}-\lambda_{c}^{*}\right)}\right]^{\frac{1}{\gamma_{p}}}$ 


\section{Optimal $c_{\text {po }}$}

\section{Derivation of solution}

The revenue is a linear function of $c_{p o}$. The constraint on $c_{p o}$ is,

$c_{p o} \leq c_{p f}+c_{p f f} \mu_{p}-c_{p t}(1+\vartheta) \alpha_{w p} \mu_{p}\left(\frac{\lambda_{p}}{n_{p} \mu_{p}}\right)^{\gamma_{p}}-c_{p t} \mu_{p} f_{d b} t_{s}\left(\lambda_{c}-\lambda_{c}^{*}\right)$

Since the problem is a linear programming, the optimal $c_{p o}$ is one of extreme points of the feasible region,

$c_{p o}^{*} \in\left\{0, c_{p f}+c_{p f f} \mu_{p}-c_{p t}(1+\vartheta) \alpha_{w p} \mu_{p}\left(\frac{\lambda_{p}}{n_{p} \mu_{p}}\right)^{\gamma_{p}}-c_{p t} \mu_{p} f_{d b} t_{s}\left(\lambda_{c}-\lambda_{c}^{*}\right)\right\}$

The revenue is an increasing function of $c_{p o}$. Therefore, the optimal solution is,

$c_{p o}^{*}=c_{p f}+c_{p f f} \mu_{p}-c_{p t}(1+\vartheta) \alpha_{w p} \mu_{p}\left(\frac{\lambda_{p}}{n_{p} \mu_{p}}\right)^{\gamma_{p}}-c_{p t} \mu_{p} f_{d b} t_{s}\left(\lambda_{c}-\lambda_{c}^{*}\right)$

\section{Optimal $c_{c o}$}

Derivation of solution

$$
\begin{aligned}
& \frac{\partial y_{r e v}}{\partial c_{c o}}=n_{c}\left(\frac{c_{v} n_{e}-c_{c o} \tau_{e}}{c_{c t} \alpha_{w c} \mu_{c} \tau_{e}}\right)^{\frac{1}{\gamma_{c}}}-\frac{n_{c} c_{c o} \tau_{e}}{\gamma_{c}\left(c_{v} n_{e}-c_{c o} \tau_{e}\right)^{\frac{\gamma_{c}-1}{\gamma_{c}}}}\left(\frac{1}{c_{c t} \alpha_{w c} \mu_{c} \tau_{e}}\right)^{\frac{1}{\gamma_{c}}}=0 \\
& c_{c o}^{*}=\frac{\gamma_{c} c_{v} n_{e}}{\left(\gamma_{c}+1\right) \tau_{e}}
\end{aligned}
$$

$\underline{\text { Optimality condition }}$

$$
\frac{\partial^{2} y_{r e v}}{\partial c_{p o}^{2}}=-\frac{\left(3 \gamma_{c}-2\right) n_{c} \tau_{e}}{\gamma_{c}\left(\gamma_{c}-1\right)\left(c_{c t} \alpha_{w c} \mu_{c} \tau_{e}\right)^{\frac{1}{\gamma_{c}}}}\left(\frac{\gamma_{c}+1}{c_{v} n_{e}}\right)^{\frac{\gamma_{c}-1}{\gamma_{c}}}<0
$$

The solution is a local maximum. 


\section{Optimal $\boldsymbol{n}_{\boldsymbol{p}}$}

\section{Derivation of solution}

The revenue is a linear function of $n_{c}$. Because of the block available length constraint, $n_{c}$ is a linear function of $n_{p}$. Therefore, the revenue is a linear function of $n_{p}$. The constraint on $n_{p}$ is,

$n_{p} \geq\left(\frac{\lambda_{p}}{\mu_{p}}\right)\left[\frac{c_{p t}(1+\vartheta) \alpha_{w p} \mu_{p}}{c_{p f}+c_{p f f} \mu_{p}-c_{p o}-c_{p t} \mu_{p} f_{d b} t_{s}\left(\lambda_{c}-\lambda_{c}^{*}\right)}\right]^{\frac{1}{\gamma_{p}}}$

Since the problem is a linear programming, the optimal $n_{p}$ is one of extreme points of the feasible region,

$n_{p}^{*} \in\left\{\left(\frac{\lambda_{p}}{\mu_{p}}\right)\left[\frac{c_{p t}(1+\vartheta) \alpha_{w p} \mu_{p}}{c_{p f}+c_{p f f} \mu_{p}-c_{p o}-c_{p t} \mu_{p} f_{d b} t_{s}\left(\lambda_{c}-\lambda_{c}^{*}\right)}\right]^{\frac{1}{\gamma_{p}}}, \quad \frac{l_{b}}{l_{p}}\right\}$

The revenue is a decreasing function of $n_{p}$. Therefore, the optimal solution is,

$n_{p}^{*}=\left(\frac{\lambda_{p}}{\mu_{p}}\right)\left[\frac{c_{p t}(1+\vartheta) \alpha_{w p} \mu_{p}}{c_{p f}+c_{p f f} \mu_{p}-c_{p o}-c_{p t} \mu_{p} f_{d b} t_{s}\left(\lambda_{c}-\lambda_{c}^{*}\right)}\right]^{\frac{1}{\gamma_{p}}}$ 


\section{$\underline{\text { Revenue model (scenario 4): formulation and derivation }}$}

\section{Scenario Information}

$\lambda_{c}^{*}<\lambda_{c}$

Some commercial vehicles double park.

$\lambda_{p}^{*}<\lambda_{p}$

Some passenger cars parking off street

\section{Cost of commercial vehicles parking}

$\underline{\text { Per-stop monetary cost of commercial vehicles parking on street }}$

$\frac{c_{c o}}{\mu_{c}}$

Per-stop waiting time of commercial vehicles parking on street

$\alpha_{w c}\left(\frac{\lambda_{c}^{*}}{n_{c} \mu_{c}}\right)^{\gamma_{c}} \quad \gamma_{c} \geq 1$

Per-stop parking search cost of commercial vehicles parking on street

Assumption: The time constraint is binding.

$c_{c t} \alpha_{w c}\left(\frac{\lambda_{c}^{*}}{n_{c} \mu_{c}}\right)^{\gamma_{c}}$

Per-stop double parking cost of commercial vehicles

$\frac{c_{v} n_{e}}{\mu_{c} \tau_{e}}$

Breakeven demand for "paying on-street" commercial parking: $\lambda_{c}^{*}$

(no impact of passengers on commercial)

$$
\begin{aligned}
& \frac{c_{c o}}{\mu_{c}}+c_{c t} \alpha_{w c}\left(\frac{\lambda_{c}^{*}}{n_{c} \mu_{c}}\right)^{\gamma_{c}}=\frac{c_{v} n_{e}}{\mu_{c} \tau_{e}} \\
& \left(\frac{\lambda_{c}^{*}}{n_{c} \mu_{c}}\right)^{\gamma_{c}}=\frac{c_{v} n_{e}-c_{c o} \tau_{e}}{c_{c t} \alpha_{w c} \mu_{c} \tau_{e}} \\
& \lambda_{c}^{*}=\frac{n_{c} \mu_{c}}{\left(c_{c t} \alpha_{w c} \mu_{c}\right)^{\frac{1}{\gamma_{c}}}}\left(\frac{c_{v} n_{e}}{\tau_{e}}-c_{c o}\right)^{\frac{1}{\gamma_{c}}}
\end{aligned}
$$




\section{Cost of passenger cars parking}

Per-stop monetary cost of a passenger car parking on street

$\frac{c_{p o}}{\mu_{p}}$

Per-stop parking waiting time of a passenger car

$\alpha_{w p}\left(\frac{\lambda_{p}^{*}}{n_{p} \mu_{p}}\right)^{\gamma_{p}} \quad \gamma_{p} \geq 1$

Per-stop parking search cost of a passenger car

Assumption: The time constraint is binding.

$c_{p t}(1+\vartheta) \alpha_{w p}\left(\frac{\lambda_{p}^{*}}{n_{p} \mu_{p}}\right)^{\gamma_{p}}$

Expected number of double-parking events that a passenger car will find while searching

$t_{s}\left(\lambda_{c}-\lambda_{c}^{*}\right)$

$\underline{\text { Per-stop delay cost of a passenger car in queue caused by double-parking commercial }}$

vehicles

$c_{p t} f_{d b} t_{s}\left(\lambda_{c}-\lambda_{c}^{*}\right)$

Per-stop parking cost of a passenger car parking off street

$\frac{c_{p f}}{\mu_{p}}+c_{p f f}$

Breakeven demand for "paying on-street" cars parking: $\lambda_{p}^{*}$

$$
\begin{aligned}
& \frac{c_{p o}}{\mu_{p}}+c_{p t}(1+\vartheta) \alpha_{w p}\left(\frac{\lambda_{p}^{*}}{n_{p} \mu_{p}}\right)^{\gamma_{p}}+c_{p t} f_{d b} t_{s}\left(\lambda_{c}-\lambda_{c}^{*}\right)=\frac{c_{p f}}{\mu_{p}}+c_{p f f} \\
& c_{p t}(1+\vartheta) \alpha_{w p}\left(\frac{\lambda_{p}^{*}}{n_{p} \mu_{p}}\right)^{\gamma_{p}}=\frac{c_{p f}}{\mu_{p}}+c_{p f f}-\frac{c_{p o}}{\mu_{p}}-c_{p t} f_{d b} t_{s}\left(\lambda_{c}-\lambda_{c}^{*}\right) \\
& \lambda_{p}^{*}=n_{p} \mu_{p}\left[\frac{1}{c_{p t}(1+\vartheta) \alpha_{w p}}\left(\frac{c_{p f}}{\mu_{p}}+c_{p f f}-\frac{c_{p o}}{\mu_{p}}-c_{p t} f_{d b} t_{s}\left(\lambda_{c}-\lambda_{c}^{*}\right)\right)\right]^{\frac{1}{\gamma_{p}}}
\end{aligned}
$$


Summary of the relevant revenue (unit: dollars per hour)

\begin{tabular}{|l|c|}
\hline Components & Formula \\
\hline Commercial parking delay cost & 0 \\
\hline Commercial maneuver traffic delay cost & 0 \\
\hline Double parking delay cost & 0 \\
\hline Passenger parking delay cost & 0 \\
\hline Passenger maneuver traffic delay cost & $\frac{c_{c o} \lambda_{c}^{*}}{\mu_{c}}$ \\
\hline On-street commercial vehicle parking revenue & $\frac{c_{p o} \lambda_{p}^{*}}{\mu_{p}}$ \\
\hline On-street passenger car parking revenue & \\
\hline
\end{tabular}

\section{Objective function}

$y_{r e v}=\frac{c_{c o} \lambda_{c}^{*}}{\mu_{c}}+\frac{c_{p o} \lambda_{p}^{*}}{\mu_{p}}$

Since $\lambda_{c}^{*}=\frac{n_{c} \mu_{c}}{\left(c_{c t} \alpha_{w c} \mu_{c}\right)^{\frac{1}{\gamma_{c}}}}\left(\frac{c_{v} n_{e}}{\tau_{e}}-c_{c o}\right)^{\frac{1}{\gamma_{c}}}$

Since $\lambda_{p}^{*}=\left[c_{p f}+c_{p f f} \mu_{p}-c_{p o}-c_{p t} f_{d b} t_{s} \mu_{p}\left\{\lambda_{c}-n_{c} \mu_{c}\left(\frac{c_{v} n_{e}-c_{c o} \tau_{e}}{c_{c t} \alpha_{w c} \mu_{c} \tau_{e}}\right)^{\frac{1}{\gamma_{c}}}\right\}\right]^{\frac{1}{\gamma_{p}}}$

$$
\frac{n_{p} \mu_{p}}{\left(c_{p t}(1+\vartheta) \alpha_{w p} \mu_{p}\right)^{\frac{1}{\gamma_{p}}}}
$$




$$
\begin{aligned}
y_{r e v}= & \frac{c_{c o} n_{c}}{\left(c_{c t} \alpha_{w c} \mu_{c}\right)^{\frac{1}{\gamma_{c}}}}\left(\frac{c_{v} n_{e}}{\tau_{e}}-c_{c o}\right)^{\frac{1}{\gamma_{c}}} \\
& +\frac{c_{p o} n_{p}}{\left(c_{p t}(1+\vartheta) \alpha_{w p} \mu_{p}\right)^{\frac{1}{\gamma_{p}}}}\left[c_{p f}+c_{p f f} \mu_{p}-c_{p o}\right. \\
& -c_{p t} f_{d b} t_{s} \mu_{p}\left\{\lambda_{c}-n_{c} \mu_{c}\left(\frac{c_{v} n_{e}-c_{c o} \tau_{e}}{c_{c t} \alpha_{w c} \mu_{c} \tau_{e}}\right)^{\frac{1}{\gamma_{c}}}\right)^{\frac{1}{\gamma_{p}}}
\end{aligned}
$$

Let $A=\left(c_{c t} \alpha_{w c} \mu_{c}\right)^{\frac{1}{\gamma_{c}}}$

Let $B=\left(c_{p t}(1+\vartheta) \alpha_{w p} \mu_{p}\right)^{\frac{1}{\gamma_{p}}}$

Let $D=c_{p f}+c_{p f f} \mu_{p}-c_{p o}-c_{p t} f_{d b} t_{s} \mu_{p}\left\{\lambda_{c}-n_{c} \mu_{c}\left(\frac{c_{v} n_{e}-c_{c o} \tau_{e}}{c_{c t} \alpha_{w c} \mu_{c} \tau_{e}}\right)^{\frac{1}{\gamma_{c}}}\right\}$

Since $A=\left(c_{c t} \alpha_{w c} \mu_{c}\right)^{\frac{1}{\gamma_{c}}}$

$D=c_{p f}+c_{p f f} \mu_{p}-c_{p o}-c_{p t} f_{d b} t_{s} \mu_{p}\left\{\lambda_{c}-\frac{n_{c} \mu_{c}}{A}\left(\frac{c_{v} n_{e}}{\tau_{e}}-c_{c o}\right)^{\frac{1}{\gamma_{c}}}\right\}$

$\frac{\partial D}{\partial c_{p o}}=-1$

$\frac{\partial D}{\partial c_{c o}}=-\frac{c_{p t} f_{d b} t_{s} \mu_{p} n_{c} \mu_{c}}{\gamma_{c} A\left(\frac{c_{v} n_{e}}{\tau_{e}}-c_{c o}\right)^{\frac{\gamma_{c}-1}{\gamma_{c}}}}$

$\frac{\partial D}{\partial n_{c}}=-\frac{c_{p t} f_{d b} t_{s} \mu_{p} \mu_{c}}{A}\left(\frac{l_{p}}{l_{c}}\right)\left(\frac{c_{v} n_{e}}{\tau_{e}}-c_{c o}\right)^{\frac{1}{\gamma_{c}}}$

$y_{r e v}=\frac{c_{c o} n_{c}}{A}\left(\frac{c_{v} n_{e}}{\tau_{e}}-c_{c o}\right)^{\frac{1}{\gamma_{c}}}+\frac{c_{p o} n_{p}}{B} D^{\frac{1}{\gamma_{p}}}$ 


\section{Optimal $c_{\text {po }}$}

$$
\begin{aligned}
& \frac{\partial y_{r e v}}{\partial c_{p o}}=\frac{n_{p}}{B} D^{\frac{1}{\gamma_{p}}}-\frac{c_{p o} n_{p}}{\gamma_{p} B}\left(D^{\frac{1-\gamma_{p}}{\gamma_{p}}}\right)=0 \\
& D-\frac{c_{p o}^{*}}{\gamma_{p}}=0
\end{aligned}
$$

Since $D=c_{p f}+c_{p f f} \mu_{p}-c_{p o}-c_{p t} f_{d b} t_{s} \mu_{p}\left\{\lambda_{c}-n_{c} \mu_{c}\left(\frac{c_{v} n_{e}-c_{c o} \tau_{e}}{c_{c t} \alpha_{w c} \mu_{c} \tau_{e}}\right)^{\frac{1}{\gamma_{c}}}\right\}$

$\left[c_{p f}+c_{p f f} \mu_{p}-c_{p o}^{*}-c_{p t} f_{d b} t_{s} \mu_{p}\left\{\lambda_{c}-n_{c} \mu_{c}\left(\frac{c_{v} n_{e}-c_{c o} \tau_{e}}{c_{c t} \alpha_{w c} \mu_{c} \tau_{e}}\right)^{\frac{1}{\gamma_{c}}}\right\}\right]^{\frac{1}{\gamma_{p}}}-\frac{c_{p o}^{*}}{\gamma_{p}}=0$

If $\gamma_{c}=\gamma_{p}=1$,

$c_{p f}+c_{p f f} \mu_{p}-c_{p o}^{*}-c_{p t} f_{d b} t_{s} \mu_{p}\left\{\lambda_{c}-n_{c} \mu_{c}\left(\frac{c_{v} n_{e}-c_{c o} \tau_{e}}{c_{c t} \alpha_{w c} \mu_{c} \tau_{e}}\right)\right\}-c_{p o}^{*}=0$

$c_{p f}+c_{p f f} \mu_{p}-c_{p t} f_{d b} t_{s} \mu_{p}\left\{\lambda_{c}-\frac{n_{c}}{c_{c t} \alpha_{w c}}\left(\frac{c_{v} n_{e}}{\tau_{e}}-c_{c o}\right)\right\}=2 c_{p o}^{*}$

$c_{p o}^{*}\left(\gamma_{c}=\gamma_{p}=1\right)=\frac{c_{p f}+c_{p f f} \mu_{p}}{2}-\frac{c_{p t} f_{d b} t_{s} \mu_{p}}{2}\left\{\lambda_{c}-\frac{n_{c}}{c_{c t} \alpha_{w c}}\left(\frac{c_{v} n_{e}}{\tau_{e}}-c_{c o}\right)\right\}$

Since, from equation (D. 1), $\quad \lambda_{c}^{*}=n_{c} \mu_{c}\left(\frac{c_{v} n_{e}-c_{c o} \tau_{e}}{c_{c t} \alpha_{w c} \mu_{c} \tau_{e}}\right)=\frac{n_{c}}{c_{c t} \alpha_{w c}}\left(\frac{c_{v} n_{e}}{\tau_{e}}-c_{c o}\right)$

$c_{p o}^{*}\left(\gamma_{c}=\gamma_{p}=1\right)=\frac{c_{p f}+c_{p f f} \mu_{p}}{2}-\frac{c_{p t}}{2}\left[f_{d b} t_{s} \mu_{p}\left(\lambda_{c}-\lambda_{c}^{*}\right)\right]$

$\underline{\text { Optimality condition }}$

$\frac{\partial^{2} y_{r e v}}{\partial c_{p o}^{2}}=-\frac{n_{p}}{\gamma_{p} B D^{\frac{\gamma_{p}-1}{\gamma_{p}}}}-\frac{n_{p}}{\gamma_{p} B D^{\frac{\gamma_{p}-1}{\gamma_{p}}}}-\frac{c_{p o} n_{p}}{\left(\gamma_{p}-1\right) B D^{\frac{-2 \gamma_{p}+1}{\gamma_{p}}}}<0$ 
The solution is a local maximum.

\section{Optimal $c_{c o}$}

$$
\begin{gathered}
\frac{\partial y_{r e v}}{\partial c_{c o}}=\frac{n_{c}}{A}\left(\frac{c_{v} n_{e}}{\tau_{e}}-c_{c o}\right)^{\frac{1}{\gamma_{c}}}-\frac{c_{c o} n_{c}}{\gamma_{c} A\left(\frac{c_{v} n_{e}}{\tau_{e}}-c_{c o}\right)^{\frac{\gamma_{c}-1}{\gamma_{c}}}}-\frac{c_{p o} n_{p} c_{p t} f_{d b} t_{s} \mu_{c} \mu_{p} n_{c}}{\gamma_{c} \gamma_{p} A B\left(\frac{c_{v} n_{e}}{\tau_{e}}-c_{c o}\right)^{\frac{\gamma_{c}-1}{\gamma_{c}}} D^{\frac{\gamma_{p}-1}{\gamma_{p}}}} \\
=0 \\
\left(\frac{c_{v} n_{e}}{\tau_{e}}-c_{c o}\right)-\frac{c_{c o}}{\gamma_{c}}-\left(\frac{c_{p o} n_{p}}{\gamma_{p} B}\right)\left(\frac{c_{p t} t_{s} \mu_{c} f_{d b} \mu_{p}}{\gamma_{c}}\right)\left(D^{\frac{1-\gamma_{p}}{\gamma_{p}}}\right)=0 \\
\gamma_{c}\left(\frac{c_{v} n_{e}}{\tau_{e}}-c_{c o}\right)-c_{c o}-c_{p t} f_{d b} t_{s} \mu_{c} \mu_{p}\left(\frac{c_{p o} n_{p}}{\gamma_{p} B}\right)\left(D^{\frac{1-\gamma_{p}}{\gamma_{p}}}\right)=0 \\
\gamma_{c}\left(\frac{c_{v} n_{e}}{\tau_{e}}\right)-\left(\gamma_{c}+1\right) c_{c o}^{*}-c_{p t} f_{d b} t_{s} \mu_{c} \mu_{p}\left(\frac{c_{p o} n_{p}}{\gamma_{p} B}\right) D^{\frac{1-\gamma_{p}}{\gamma_{p}}}=0 \\
c_{c o}^{*}\left(\gamma_{c}=\gamma_{p}=1\right)=\frac{c_{v} n_{e}}{2 \tau_{e}}-\frac{f_{d b} t_{s} \mu_{c} n_{p} c_{p o}}{2(1+\vartheta) \alpha_{w p}} \quad(D .8) \\
\text { If } \gamma_{c}=\gamma_{p}=1,
\end{gathered}
$$

\section{$\underline{\text { Optimality condition }}$}

$$
\begin{aligned}
\frac{\partial^{2} y_{r e v}}{\partial c_{c o}^{2}}=-\left(\gamma_{c}\right. & +1)-\frac{\gamma_{p}}{\gamma_{p}-1} c_{p t} f_{d b} t_{s} \mu_{c} \mu_{p}\left(\frac{c_{p o} n_{p}}{\gamma_{p} B}\right)\left(D^{\frac{1-2 \gamma_{p}}{\gamma_{p}}}\right) \cdot \frac{c_{p t} f_{d b} t_{s} \mu_{c} \mu_{p} n_{c}}{\gamma_{c} A\left(\frac{c_{v} n_{e}}{\tau_{e}}-c_{c o}\right)^{\frac{\gamma_{c}-1}{\gamma_{c}}}} \\
& <0
\end{aligned}
$$

The solution is a local maximum. 


\section{Optimal $\boldsymbol{n}_{\boldsymbol{p}}$}

Let $\gamma_{c}=\gamma_{p}=1, E q(D .3)$ becomes,

$$
\begin{aligned}
y_{r e v}= & c_{c o}\left(\frac{l_{b}-n_{p} l_{p}}{l_{c}}\right)\left(\frac{c_{v} n_{e}-c_{c o} \tau_{e}}{c_{c t} \alpha_{w c} \mu_{c} \tau_{e}}\right) \\
& +\frac{n_{p} c_{p o}}{(1+\vartheta) \alpha_{w p}}\left\{\frac{\left(c_{p f}+c_{p f f} \mu_{p}-c_{p o}\right)}{c_{p t} \mu_{p}}-f_{d b} t_{s}\left[\lambda_{c}-\frac{n_{c}}{c_{c t} \alpha_{w c}}\left(\frac{c_{v} n_{e}}{\tau_{e}}-c_{c o}\right)\right]\right\} \\
\frac{\partial y_{r e v}}{\partial n_{p}}=0=-c_{c o}\left(\frac{l_{p}}{l_{c}}\right)\left(\frac{c_{v} n_{e}-c_{c o} \tau_{e}}{c_{c t} \alpha_{w c} \mu_{c} \tau_{e}}\right) & \\
& +\frac{c_{p o}}{(1+\vartheta) \alpha_{w p}}\left\{\left(\frac{c_{p f}+c_{p f f} \mu_{p}-c_{p o}}{c_{p t} \mu_{p}}\right)-f_{d b} t_{s} \lambda_{c}\right. \\
& \left.+\frac{f_{d b} t_{s}}{c_{c t} \alpha_{w c}}\left(\frac{l_{b}-2 n_{p} l_{p}}{l_{c}}\right)\left(\frac{c_{v} n_{e}}{\tau_{e}}-c_{c o}\right)\right\}
\end{aligned}
$$

$$
\begin{aligned}
\left(\frac{1}{\mu_{c}}\right)\left(\frac{c_{c o}}{c_{p o}}\right)\left(\frac{l_{p}}{l_{c}}\right) & \left(\frac{c_{v} n_{e}-c_{c o} \tau_{e}}{c_{c t} \tau_{e}}\right)\left(\frac{(1+\vartheta) \alpha_{w p}}{\alpha_{w c}}\right)+f_{d b} t_{s} \lambda_{c} \\
- & \left(\frac{1}{\mu_{p}}\right)\left(\frac{c_{p f}+c_{p f f} \mu_{p}-c_{p o}}{c_{p t}}\right)=\frac{f_{d b} t_{s}}{\alpha_{w c}}\left(\frac{l_{b}-2 n_{p} l_{p}}{l_{c}}\right)\left(\frac{c_{v} n_{e}-c_{c o} \tau_{e}}{c_{c t} \tau_{e}}\right)
\end{aligned}
$$

$$
\begin{aligned}
\left(\frac{c_{c o}}{c_{p o}}\right)\left(\frac{l_{p}}{l_{c}}\right)\left(\frac{(1+\vartheta) \alpha_{w p}}{f_{d b} t_{s} \mu_{c}}\right)+\lambda_{c} \alpha_{w c}\left(\frac{c_{c t} \tau_{e}}{c_{v} n_{e}-c_{c o} \tau_{e}}\right) \\
\quad-\left(\frac{\alpha_{w c}}{f_{d b} t_{s} \mu_{p}}\right)\left(\frac{c_{p f}+c_{p f f} \mu_{p}-c_{p o}}{c_{p t}}\right)\left(\frac{c_{c t} \tau_{e}}{c_{v} n_{e}-c_{c o} \tau_{e}}\right)=\frac{l_{b}-2 n_{p} l_{p}}{l_{c}}
\end{aligned}
$$




$$
\begin{aligned}
n_{p}^{*}\left(\gamma_{c}=\gamma_{p}=1\right) & \\
= & \frac{l_{b}}{2 l_{p}}-\lambda_{c} \alpha_{w c}\left(\frac{l_{c}}{2 l_{p}}\right)\left(\frac{c_{c t} \tau_{e}}{c_{v} n_{e}-c_{c o} \tau_{e}}\right)-\frac{1}{2}\left(\frac{c_{c o}}{c_{p o}}\right)\left(\frac{(1+\vartheta) \alpha_{w p}}{f_{d b} t_{s} \mu_{c}}\right) \\
& +\left(\frac{l_{c}}{2 l_{p}}\right)\left(\frac{\alpha_{w c}}{f_{d b} t_{s} \mu_{p}}\right)\left(\frac{c_{p f}+c_{p f f} \mu_{p}-c_{p o}}{c_{p t}}\right)\left(\frac{c_{c t} \tau_{e}}{c_{v} n_{e}-c_{c o} \tau_{e}}\right)
\end{aligned}
$$

\section{Optimality condition}

$\frac{\partial^{2} y_{r e v}}{\partial n_{p}^{2}}=-\frac{c_{p o}}{(1+\vartheta) \alpha_{w p}} \frac{f_{d b} t_{s}}{c_{c t} \alpha_{w c}}\left(\frac{2 l_{p}}{l_{c}}\right)\left(\frac{c_{v} n_{e}}{\tau_{e}}-c_{c o}\right)<0$

The solution is a local maximum. 


\section{$\underline{\text { Social optimum (SOC) model (scenario 1): formulation, derivation }}$}

\section{Scenario information}

$\lambda_{c}^{*} \geq \lambda_{c}$

No commercial vehicles double parking

$\lambda_{p}^{*} \geq \lambda_{p}$

No cars parking off street

\section{Revenue and cost of commercial vehicles parking}

$\underline{\text { Per-stop monetary cost of a commercial vehicle parking on street }}$

$\frac{c_{c o}}{\mu_{c}}$

$\underline{\text { Per-stop waiting time of a commercial vehicle parking on street }}$

$\alpha_{w c}\left(\frac{\lambda_{c}}{n_{c} \mu_{c}}\right)^{\gamma_{c}} \quad \gamma_{c} \geq 1$

$\underline{\text { Per-stop parking delay cost of a commercial vehicle parking on street }}$

$c_{c t} \alpha_{w c}\left(\frac{\lambda_{c}}{n_{c} \mu_{c}}\right)^{\gamma_{c}}$

Per-stop double parking cost of a commercial vehicle

$\frac{c_{v} n_{e}}{\mu_{c} \tau_{e}}$

$\underline{\text { Relationship between on-street parking and double parking }}$

$$
\begin{aligned}
& \frac{c_{c o}}{\mu_{c}}+c_{c t} \alpha_{w c}\left(\frac{\lambda_{c}}{n_{c} \mu_{c}}\right)^{\gamma_{c}} \leq \frac{c_{v} n_{e}}{\mu_{c} \tau_{e}} \\
& c_{c o} \leq \frac{c_{v} n_{e}}{\tau_{e}}-c_{c t} \alpha_{w c} \mu_{c}\left(\frac{\lambda_{c}}{n_{c} \mu_{c}}\right)^{\gamma_{c}} \\
& n_{c} \geq\left(\frac{\lambda_{c}}{\mu_{c}}\right)\left(\frac{c_{c t} \alpha_{w c} \mu_{c}}{\frac{c_{v} n_{e}}{\tau_{e}}-c_{c o}}\right)^{\frac{1}{\gamma_{c}}}
\end{aligned}
$$




\section{Revenue and cost of passenger cars parking}

Per-stop monetary cost of a passenger car parking on street

$\frac{c_{p o}}{\mu_{p}}$

Per-stop parking waiting time of a passenger car

$\alpha_{w p}\left(\frac{\lambda_{p}}{n_{p} \mu_{p}}\right)^{\gamma_{p}} \quad \gamma_{p} \geq \gamma_{c} \geq 1$

Per-stop parking delay cost of a passenger car

$c_{p t}(1+\vartheta) \alpha_{w p}\left(\frac{\lambda_{p}}{n_{p} \mu_{p}}\right)^{\gamma_{p}}$

Per-stop parking cost of a passenger car parking off street

$\frac{c_{p f}}{\mu_{p}}+c_{p f f}$

$\underline{\text { Relationship between on-street parking and double parking }}$

$$
\begin{aligned}
& \frac{c_{p o}}{\mu_{p}}+c_{p t}(1+\vartheta) \alpha_{w p}\left(\frac{\lambda_{p}}{n_{p} \mu_{p}}\right)^{\gamma_{p}} \leq \frac{c_{p f}}{\mu_{p}}+c_{p f f} \\
& c_{p o} \leq c_{p f}+c_{p f f} \mu_{p}-c_{p t}(1+\vartheta) \alpha_{w p} \mu_{p}\left(\frac{\lambda_{p}}{n_{p} \mu_{p}}\right)^{\gamma_{p}} \\
& n_{p} \geq\left(\frac{\lambda_{p}}{\mu_{p}}\right)\left[\frac{c_{p t}(1+\vartheta) \alpha_{w p} \mu_{p}}{c_{p f}+c_{p f f} \mu_{p}-c_{p o}}\right]^{\frac{1}{\gamma_{p}}}
\end{aligned}
$$


Summary of the relevant revenue and cost (unit: dollars per hour)

\begin{tabular}{|l|c|}
\hline Components & Formula \\
\hline Commercial parking delay cost & $c_{c t} \alpha_{w c}\left(\frac{\lambda_{c}}{n_{c} \mu_{c}}\right)^{\gamma_{c}} \lambda_{c}$ \\
\hline Commercial maneuver traffic delay cost & $c_{d c} \lambda_{c}$ \\
\hline Double parking delay cost & 0 \\
\hline Passenger parking delay cost & $c_{p t}(1+\vartheta) \alpha_{w p}\left(\frac{\lambda_{p}}{n_{p} \mu_{p}}\right)^{\gamma_{p}} \lambda_{p}$ \\
\hline Passenger maneuver traffic delay cost & $c_{d p} \lambda_{p}$ \\
\hline On-street commercial vehicle parking revenue & $\frac{c_{c o} \lambda_{c}}{\mu_{c}}$ \\
\hline On-street passenger vehicle parking revenue & $\frac{c_{p o} \lambda_{p}}{\mu_{p}}$ \\
\hline
\end{tabular}

\section{Objective function}

$$
\begin{aligned}
y_{s o c}=\frac{c_{c o} \lambda_{c}}{\mu_{c}}+ & \frac{c_{p o} \lambda_{p}}{\mu_{p}}-c_{c t} \alpha_{w c}\left(\frac{\lambda_{c}}{n_{c} \mu_{c}}\right)^{\gamma_{c}} \lambda_{c}-c_{p t}(1+\vartheta) \alpha_{w p}\left(\frac{\lambda_{p}}{n_{p} \mu_{p}}\right)^{\gamma_{p}} \lambda_{p}-c_{d c} \lambda_{c} \\
& -c_{d p} \lambda_{p}
\end{aligned}
$$




\section{Optimal $c_{\text {po }}$}

\section{Derivation of solution}

The revenue is a linear function of $c_{p o}$. The constraint on $c_{p o}$ is,

$c_{p o} \leq c_{p f}+c_{p f f} \mu_{p}-c_{p t}(1+\vartheta) \alpha_{w p} \mu_{p}\left(\frac{\lambda_{p}}{n_{p} \mu_{p}}\right)^{\gamma_{p}}$

Since the problem is a linear programming, the optimal $c_{p o}$ is one of extreme points of the feasible region,

$c_{p o}^{*} \in\left\{0, c_{p f}+c_{p f f} \mu_{p}-c_{p t}(1+\vartheta) \alpha_{w p} \mu_{p}\left(\frac{\lambda_{p}}{n_{p} \mu_{p}}\right)^{\gamma_{p}}\right\}$

The revenue is an increasing function of $c_{p o}$. Therefore, the optimal solution is,

$c_{p o}^{*}=c_{p f}+c_{p f f} \mu_{p}-c_{p t}(1+\vartheta) \alpha_{w p} \mu_{p}\left(\frac{\lambda_{p}}{n_{p} \mu_{p}}\right)^{\gamma_{p}}$

\section{Optimal $c_{c o}$}

\section{$\underline{\text { Derivation of solution }}$}

The revenue is a linear function of $c_{c o}$. The constraint on $c_{c o}$ is,

$c_{c o} \leq \frac{c_{v} n_{e}}{\tau_{e}}-c_{c t} \alpha_{w c} \mu_{c}\left(\frac{\lambda_{c}}{n_{c} \mu_{c}}\right)^{\gamma_{c}}$

Since the problem is a linear programming, the optimal $c_{c o}$ is one of extreme points of the feasible region,

$c_{c o}^{*} \in\left\{0, \frac{c_{v} n_{e}}{\tau_{e}}-c_{c t} \alpha_{w c} \mu_{c}\left(\frac{\lambda_{c}}{n_{c} \mu_{c}}\right)^{\gamma_{c}}\right\}$

The revenue is an increasing function of $c_{c o}$. Therefore, the optimal solution is,

$c_{c o}^{*}=\frac{c_{v} n_{e}}{\tau_{e}}-c_{c t} \alpha_{w c} \mu_{c}\left(\frac{\lambda_{c}}{n_{c} \mu_{c}}\right)^{\gamma_{c}}$ 


\section{Optimal $\boldsymbol{n}_{\boldsymbol{p}}$}

\section{Derivation of solution}

$$
\begin{aligned}
& \text { Let } \gamma_{c}=\gamma_{p}=1, \\
& \text { Since } n_{c}=\frac{l_{b}-n_{p} l_{p}}{l_{c}} \\
& \begin{aligned}
y_{n e t}=\frac{c_{c o} \lambda_{c}}{\mu_{c}}+\frac{c_{p o} \lambda_{p}}{\mu_{p}}-c_{c t} \alpha_{w c}\left(\frac{\lambda_{c}^{2}}{\mu_{c}}\right)\left(\frac{l_{c}}{l_{b}-n_{p} l_{p}}\right)-c_{d c} \lambda_{c}-c_{p t}(1+\vartheta) \alpha_{w p}\left(\frac{\lambda_{p}^{2}}{n_{p} \mu_{p}}\right) \\
\quad-c_{d p} \lambda_{p}
\end{aligned}
\end{aligned}
$$

$$
\begin{aligned}
& \frac{\partial y_{n e t}}{\partial n_{p}}=-c_{c t} \alpha_{w c}\left(\frac{\lambda_{c}^{2}}{\mu_{c}}\right) \frac{l_{c} l_{p}}{\left(l_{b}-n_{p} l_{p}\right)^{2}}+c_{p t}(1+\vartheta) \alpha_{w p}\left(\frac{\lambda_{p}^{2}}{\mu_{p}}\right) \frac{1}{n_{p}^{2}}=0 \\
& \frac{\left(l_{b}-n_{p} l_{p}\right)^{2}}{n_{p}^{2}}=l_{c} l_{p}\left(\frac{c_{c t} \alpha_{w c}}{c_{p t}(1+\vartheta) \alpha_{w p}}\right)\left(\frac{\lambda_{c}^{2}}{\lambda_{p}^{2}}\right)\left(\frac{\mu_{p}}{\mu_{c}}\right) \\
& l_{b}-n_{p} l_{p}=n_{p}\left(\frac{\lambda_{c}}{\lambda_{p}}\right) \sqrt{l_{c} l_{p}\left(\frac{c_{c t} \alpha_{w c}}{c_{p t}(1+\vartheta) \alpha_{w p}}\right)\left(\frac{\mu_{p}}{\mu_{c}}\right)} \\
& n_{p}^{*}\left(\gamma_{c}=\gamma_{p}=1\right)=\frac{l_{b}}{l_{p}+\left(\frac{\lambda_{c}}{\lambda_{p}}\right) \sqrt{l_{c} l_{p} \frac{c_{c t} \alpha_{w c}}{c_{p t}(1+\vartheta) \alpha_{w p}}\left(\frac{\mu_{p}}{\mu_{c}}\right)}}
\end{aligned}
$$

Optimality condition

$$
\frac{\partial^{2} y_{s o c}\left(\gamma_{c}=\gamma_{p}=1\right)}{\partial n_{p}^{2}}=-2 c_{c t} \alpha_{w c}\left(\frac{\lambda_{c}^{2}}{\mu_{c}}\right) \frac{l_{c} l_{p}^{2}}{\left(l_{b}-n_{p} l_{p}\right)^{3}}-2 c_{p t}(1+\vartheta) \alpha_{w p}\left(\frac{\lambda_{p}^{2}}{\mu_{p}}\right) \frac{1}{n_{p}^{3}}<0
$$

The solution is a local maximum. 


\section{$\underline{\text { Social optimum (SOC) model (scenario 2): formulation, derivation }}$}

\section{Scenario information}

$\lambda_{c}^{*} \geq \lambda_{c}$

All the commercial vehicles parking on street

$\lambda_{p}^{*}<\lambda_{p}$

Some cars parking off street

\section{Revenue and cost of commercial vehicles parking}

$\underline{\text { Per-stop monetary cost of a commercial vehicle parking on street }}$

$\frac{c_{c o}}{\mu_{c}}$

Per-stop waiting time of a commercial vehicle parking on street

$\alpha_{w c}\left(\frac{\lambda_{c}}{n_{c} \mu_{c}}\right)^{\gamma_{c}} \quad \gamma_{c} \geq 1$

$\underline{\text { Per-stop parking delay cost of a commercial vehicle parking on street }}$

$c_{c t} \alpha_{w c}\left(\frac{\lambda_{c}}{n_{c} \mu_{c}}\right)^{\gamma_{c}}$

Per-stop double parking cost of a commercial vehicle

$\frac{c_{v} n_{e}}{\mu_{c} \tau_{e}}$

$\underline{\text { Relationship between on-street parking and double parking }}$

$$
\begin{aligned}
& \frac{c_{c o}}{\mu_{c}}+c_{c t} \alpha_{w c}\left(\frac{\lambda_{c}}{n_{c} \mu_{c}}\right)^{\gamma_{c}} \leq \frac{c_{v} n_{e}}{\mu_{c} \tau_{e}} \\
& c_{c o} \leq \frac{c_{v} n_{e}}{\tau_{e}}-c_{c t} \alpha_{w c} \mu_{c}\left(\frac{\lambda_{c}}{n_{c} \mu_{c}}\right)^{\gamma_{c}} \\
& n_{c} \geq\left(\frac{\lambda_{c}}{\mu_{c}}\right)\left(\frac{c_{c t} \alpha_{w c} \mu_{c}}{\frac{c_{v} n_{e}}{\tau_{e}}-c_{c o}}\right)^{\frac{1}{\gamma_{c}}}
\end{aligned}
$$




\section{Revenue and cost of passenger cars parking}

Per-stop monetary cost of a passenger car parking on street

$\frac{c_{p o}}{\mu_{p}}$

Per-stop parking waiting time of a passenger car

$\alpha_{w p}\left(\frac{\lambda_{p}^{*}}{n_{p} \mu_{p}}\right)^{\gamma_{p}} \quad \gamma_{p} \geq \gamma_{c} \geq 1$

Per-stop parking delay cost of a passenger car

$c_{p t}(1+\vartheta) \alpha_{w p}\left(\frac{\lambda_{p}^{*}}{n_{p} \mu_{p}}\right)^{\gamma_{p}}$

$\underline{\text { Per-stop parking cost of a passenger car parking off street }}$

$\frac{c_{p f}}{\mu_{p}}+c_{p f f}$

Breakeven demand for "paying on-street" passenger parking: $\lambda_{p}^{*} \mathrm{~W}$

$$
\begin{aligned}
& \frac{c_{p o}}{\mu_{p}}+c_{p t}(1+\vartheta) \alpha_{w p}\left(\frac{\lambda_{p}^{*}}{n_{p} \mu_{p}}\right)^{\gamma_{p}}=\frac{c_{p f}}{\mu_{p}}+c_{p f f} \\
& c_{p t}(1+\vartheta) \alpha_{w p}\left(\frac{\lambda_{p}^{*}}{n_{p} \mu_{p}}\right)^{\gamma_{p}}=\frac{c_{p f}}{\mu_{p}}+c_{p f f}-\frac{c_{p o}}{\mu_{p}} \\
& \left(\frac{\lambda_{p}^{*}}{n_{p} \mu_{p}}\right)^{\gamma_{p}}=\frac{c_{p f}+c_{p f f} \mu_{p}-c_{p o}}{c_{p t}(1+\vartheta) \alpha_{w p} \mu_{p}} \\
& \lambda_{p}^{*}=n_{p} \mu_{p}\left[\frac{c_{p f}+c_{p f f} \mu_{p}-c_{p o}}{c_{p t}(1+\vartheta) \alpha_{w p} \mu_{p}}\right]^{\frac{1}{\gamma_{p}}}
\end{aligned}
$$


Summary of the relevant revenue and cost (unit: dollars per hour)

\begin{tabular}{|l|c|}
\hline Components & Formula \\
\hline Commercial parking delay cost & $c_{c t} \alpha_{w c}\left(\frac{\lambda_{c}}{n_{c} \mu_{c}}\right)^{\gamma_{c}} \lambda_{c}$ \\
\hline Commercial maneuver traffic delay cost & $c_{d c} \lambda_{c}$ \\
\hline Double parking delay cost & 0 \\
\hline Passenger parking delay cost & $c_{p t}(1+\vartheta) \alpha_{w p}\left(\frac{\lambda_{p}^{*}}{n_{p} \mu_{p}}\right)^{\gamma_{p}} \lambda_{p}$ \\
\hline Passenger maneuver traffic delay cost & $\frac{c_{d p} \lambda_{p}^{*}}{c_{c o} \lambda_{c}}$ \\
\hline On-street commercial vehicle parking revenue & $\frac{c_{p o} \lambda_{p}^{*}}{\mu_{p}}$ \\
\hline On-street passenger vehicle parking revenue & \\
\hline
\end{tabular}

\section{Objective function}

$$
\begin{aligned}
y_{s o c}=\frac{c_{c o} \lambda_{c}}{\mu_{c}}+ & \frac{c_{p o} \lambda_{p}^{*}}{\mu_{p}}-c_{c t} \alpha_{w c}\left(\frac{\lambda_{c}}{n_{c} \mu_{c}}\right)^{\gamma_{c}} \lambda_{c}-c_{p t}(1+\vartheta) \alpha_{w p}\left(\frac{\lambda_{p}^{*}}{n_{p} \mu_{p}}\right)^{\gamma_{p}} \lambda_{p}-c_{d c} \lambda_{c} \\
& -c_{d p} \lambda_{p}^{*}
\end{aligned}
$$

From equation (F.3), the objective function becomes,

$$
\begin{gathered}
y_{s o c}=\frac{c_{c o} \lambda_{c}}{\mu_{c}}-c_{c t} \alpha_{w c}\left(\frac{\lambda_{c}}{n_{c} \mu_{c}}\right)^{\gamma_{c}} \lambda_{c}-c_{d c} \lambda_{c}+\frac{n_{p}\left(c_{p o}-c_{d p} \mu_{p}\right)\left(c_{p f}+c_{p f f} \mu_{p}-c_{p o}\right)^{\frac{1}{\gamma_{p}}}}{\left[c_{p t}(1+\vartheta) \alpha_{w p} \mu_{p}\right]^{\frac{1}{\gamma_{p}}}} \\
-\left(c_{p f}+c_{p f f} \mu_{p}-c_{p o}\right) \frac{\lambda_{p}}{\mu_{p}}
\end{gathered}
$$




\section{Optimal $c_{p o}$}

\section{Derivation of solution}

$\frac{\partial y_{s o c}}{\partial c_{p o}}=0$

$\frac{n_{p}}{\left[c_{p t}(1+\vartheta) \alpha_{w p} \mu_{p}\right]^{\frac{1}{\gamma_{p}}}}\left[\left(c_{p f}+c_{p f f} \mu_{p}-c_{p o}\right)^{\frac{1}{\gamma_{p}}}-\frac{\left(c_{p o}-c_{d p} \mu_{p}\right)}{\gamma_{p}\left(c_{p f}+c_{p f f} \mu_{p}-c_{p o}\right)^{\frac{\gamma_{p}-1}{\gamma_{p}}}}\right]+\frac{\lambda_{p}}{\mu_{p}}$
$=0$

$\frac{n_{p}\left[\gamma_{p}\left(c_{p f}+c_{p f f} \mu_{p}\right)-c_{p o}^{*}\left(\gamma_{p}+1\right)+c_{d p} \mu_{p}\right]}{\left[c_{p t}(1+\vartheta) \alpha_{w p} \mu_{p}\right]^{\frac{1}{\gamma_{p}}} \gamma_{p}\left(c_{p f}+c_{p f f} \mu_{p}-c_{p o}^{*}\right)^{\frac{\gamma_{p}-1}{\gamma_{p}}}}+\frac{\lambda_{p}}{\mu_{p}}=0$

If $\gamma_{p}=1$,

$\frac{n_{p}\left[\left(c_{p f}+c_{p f f} \mu_{p}\right)-2 c_{p o}+c_{d p} \mu_{p}\right]}{c_{p t}(1+\vartheta) \alpha_{w p} \mu_{p}}+\frac{\lambda_{p}}{\mu_{p}}=0$

$c_{p o}^{*}\left(\gamma_{p}=1\right)=\frac{c_{p f}+c_{p f f} \mu_{p}}{2}+\frac{c_{d p} \mu_{p}}{2}+\frac{c_{p t}(1+\vartheta) \alpha_{w p}}{2}\left(\frac{\lambda_{p}}{n_{p}}\right)$

Check the optimality condition

$\frac{\partial^{2} y_{s o c}}{\partial c_{p o}^{2}}=-\frac{n_{p}\left[2 \gamma_{p}\left(c_{p f}+c_{p f f} \mu_{p}\right)-\left(\gamma_{p}+1\right) c_{p o}-\left(\gamma_{p}-1\right) c_{d p} \mu_{p}\right]}{\gamma_{p}^{2}\left[c_{p t}(1+\vartheta) \alpha_{w p} \mu_{p}\right]^{\frac{1}{\gamma_{p}}}\left(c_{p f}+c_{p f f} \mu_{p}-c_{p o}\right)^{\frac{2 \gamma_{p}-1}{\gamma_{p}}}}<0$

Therefore, the solution is a local maximum. 


\section{Optimal $c_{c o}$}

Derivation of solution

The social optimum is a linear function of $c_{c o}$. The constraint on $c_{c o}$ is,

$c_{c o} \leq \frac{c_{v} n_{e}}{\tau_{e}}-c_{c t} \alpha_{w c} \mu_{c}\left(\frac{\lambda_{c}}{n_{c} \mu_{c}}\right)^{\gamma_{c}}$

Since the problem is a linear programming, the optimal $c_{c o}$ is one of extreme points of the feasible region,

$c_{c o}^{*} \in\left\{0, \frac{c_{v} n_{e}}{\tau_{e}}-c_{c t} \alpha_{w c} \mu_{c}\left(\frac{\lambda_{c}}{n_{c} \mu_{c}}\right)^{\gamma_{c}}\right\}$

The social optimum is an increasing function of $c_{c o}$. Therefore, the optimal solution is,

$c_{c o}^{*}=\frac{c_{v} n_{e}}{\tau_{e}}-c_{c t} \alpha_{w c} \mu_{c}\left(\frac{\lambda_{c}}{n_{c} \mu_{c}}\right)^{\gamma_{c}}$ 


\section{Optimal $\boldsymbol{n}_{\boldsymbol{p}}$}

\section{Derivation of solution}

Let $\gamma_{c}=\gamma_{p}=1$,

Since $n_{c}=\frac{l_{b}-n_{p} l_{p}}{l_{c}}$,

$$
\begin{aligned}
y_{s o c}=\frac{c_{c o} \lambda_{c}}{\mu_{c}}+ & n_{p}\left(\frac{c_{p f}+c_{p f f} \mu_{p}-c_{p o}}{c_{p t}(1+\vartheta) \alpha_{w p}}\right)\left[\frac{c_{p o}}{\mu_{p}}-c_{p t}(1+\vartheta) \alpha_{w p}\left(\frac{\lambda_{p}}{n_{p} \mu_{p}}\right)-c_{d p}\right] \\
& -\frac{c_{c t} \alpha_{w c} \lambda_{c}^{2}}{\mu_{c}}\left(\frac{l_{c}}{l_{b}-n_{p} l_{p}}\right)-c_{d c} \lambda_{c}
\end{aligned}
$$

$$
\begin{aligned}
& \frac{\partial y_{s o c}}{\partial n_{p}}=\left(\frac{c_{p f}+c_{p f f} \mu_{p}-c_{p o}}{c_{p t}(1+\vartheta) \alpha_{w p}}\right)\left[\frac{c_{p o}}{\mu_{p}}-c_{d p}\right]-\frac{c_{c t} \alpha_{w c} \lambda_{c}^{2} l_{c} l_{p}}{\mu_{c}\left(l_{b}-n_{p} l_{p}\right)^{2}}=0 \\
& \left(l_{b}-n_{p} l_{p}\right)^{2}=\frac{\lambda_{c}^{2}\left(c_{c t} \alpha_{w c}\right)\left[c_{p t}(1+\vartheta) \alpha_{w p}\right] l_{c} l_{p}}{\left(c_{p f}+c_{p f f} \mu_{p}-c_{p o}\right)\left(c_{p o}-c_{d p} \mu_{p}\right)}\left(\frac{\mu_{p}}{\mu_{c}}\right) \\
& n_{p}^{*}\left(\gamma_{c}=\gamma_{p}=1\right)=\frac{1}{l_{p}}\left[l_{b}-\lambda_{c} \sqrt{\left(\frac{\mu_{p}}{\mu_{c}}\right) \frac{\left(c_{c t} \alpha_{w c}\right)\left(c_{p t}(1+\vartheta) \alpha_{w p}\right) l_{c} l_{p}}{\left(c_{p f}+c_{p f f} \mu_{p}-c_{p o}\right)\left(c_{p o}-c_{d p} \mu_{p}\right)}}\right]
\end{aligned}
$$

\section{Check the optimality condition}

$\frac{\partial^{2} y_{s o c}}{\partial n_{p}^{2}}=-\frac{2 c_{c t} \alpha_{w c} \lambda_{c}^{2} l_{c} l_{p}^{2}}{\mu_{c}\left(l_{b}-n_{p} l_{p}\right)^{3}}<0$

Therefore, the solution is a local maximum. 


\section{$\underline{\text { Social optimum (SOC) model (scenario 3): formulation and derivation }}$}

\section{Scenario Information}

$\lambda_{c}^{*}<\lambda_{c}$

Some commercial vehicles double park.

$\lambda_{p}^{*} \geq \lambda_{p}$

No cars parking off street

\section{Cost of commercial vehicles parking}

$\underline{\text { Per-stop monetary cost of commercial vehicles parking on street }}$

$\frac{c_{c o}}{\mu_{c}}$

$\underline{\text { Per-stop waiting time of commercial vehicles parking on street }}$

$\alpha_{w c}\left(\frac{\lambda_{c}^{*}}{n_{c} \mu_{c}}\right)^{\gamma_{c}} \quad \gamma_{c} \geq 1$

$\underline{\text { Per-stop parking search cost of commercial vehicles parking on street }}$

Assumption: The time constraint is binding.

$c_{c t} \alpha_{w c}\left(\frac{\lambda_{c}^{*}}{n_{c} \mu_{c}}\right)^{\gamma_{c}}$

$\underline{\text { Per-stop double parking cost of commercial vehicles }}$

$\frac{c_{v} n_{e}}{\mu_{c} \tau_{e}}$

Breakeven demand for "paying on-street" commercial parking: $\lambda_{c}^{*}$

(no impact of passengers on commercial)

$\frac{c_{c o}}{\mu_{c}}+c_{c t} \alpha_{w c}\left(\frac{\lambda_{c}^{*}}{n_{c} \mu_{c}}\right)^{\gamma_{c}}=\frac{c_{v} n_{e}}{\mu_{c} \tau_{e}}$

$\left(\frac{\lambda_{c}^{*}}{n_{c} \mu_{c}}\right)^{\gamma_{c}}=\frac{c_{v} n_{e}-c_{c o} \tau_{e}}{c_{c t} \alpha_{w c} \mu_{c} \tau_{e}}$

$\lambda_{c}^{*}=n_{c} \mu_{c}\left(\frac{c_{v} n_{e}-c_{c o} \tau_{e}}{c_{c t} \alpha_{w c} \mu_{c} \tau_{e}}\right)^{\frac{1}{\gamma_{c}}}$ 


\section{Cost of passenger cars parking}

Per-stop monetary cost of a passenger car parking on street

$\frac{c_{p o}}{\mu_{p}}$

Per-stop parking waiting time of a passenger car

$\alpha_{w p}\left(\frac{\lambda_{p}^{*}}{n_{p} \mu_{p}}\right)^{\gamma_{p}} \quad \gamma_{p} \geq \gamma_{c} \geq 1$

Per-stop parking search cost of a passenger car

Assumption: The time constraint is binding.

$c_{p t}(1+\vartheta) \alpha_{w p}\left(\frac{\lambda_{p}^{*}}{n_{p} \mu_{p}}\right)^{\gamma_{p}}$

Expected number of double-parking events that a passenger car will find while searching

$t_{s}\left(\lambda_{c}-\lambda_{c}^{*}\right)$

$\underline{\text { Per-stop delay cost of a passenger car in queue caused by double-parking commercial }}$

vehicles

$c_{p t} f_{d b} t_{s}\left(\lambda_{c}-\lambda_{c}^{*}\right)$

Per-stop parking cost of a passenger car parking off street

$\frac{c_{p f}}{\mu_{p}}+c_{p f f}$

$\underline{\text { Relationship between on-street parking and double parking }}$

$$
\begin{aligned}
& \frac{c_{p o}}{\mu_{p}}+c_{p t}(1+\vartheta) \alpha_{w p}\left(\frac{\lambda_{p}}{n_{p} \mu_{p}}\right)^{\gamma_{p}}+c_{p t} f_{d b} t_{s}\left(\lambda_{c}-\lambda_{c}^{*}\right) \leq \frac{c_{p f}}{\mu_{p}}+c_{p f f} \\
& c_{p o} \leq c_{p f}+c_{p f f} \mu_{p}-c_{p t}(1+\vartheta) \alpha_{w p} \mu_{p}\left(\frac{\lambda_{p}}{n_{p} \mu_{p}}\right)^{\gamma_{p}}-c_{p t} \mu_{p} f_{d b} t_{s}\left(\lambda_{c}-\lambda_{c}^{*}\right) \\
& n_{p} \geq \frac{\lambda_{p}}{\mu_{p}}\left[\frac{c_{p t}(1+\vartheta) \alpha_{w p} \mu_{p}}{c_{p f}+c_{p f f} \mu_{p}-c_{p o}-c_{p t} f_{d b} t_{s} \mu_{p}\left(\lambda_{c}-\lambda_{c}^{*}\right)}\right]^{\frac{1}{\gamma_{p}}}
\end{aligned}
$$


Summary of the relevant revenue and cost (unit: dollars per hour)

\begin{tabular}{|l|c|}
\hline Components & Formula \\
\hline Commercial parking delay cost & $c_{c t} \alpha_{w c}\left(\frac{\lambda_{c}^{*}}{n_{c} \mu_{c}}\right)^{\gamma_{c}} \lambda_{c}$ \\
\hline Commercial maneuver traffic delay cost & $c_{d c} \lambda_{c}^{*}$ \\
\hline Double parking delay cost & $c_{d b}\left(\lambda_{c}-\lambda_{c}^{*}\right)$ \\
\hline Car parking delay cost & {$\left[c_{p t}(1+\vartheta) \alpha_{w p}\left(\frac{\lambda_{p}}{n_{p} \mu_{p}}\right)^{\gamma_{p}}\right.$} \\
\hline Car maneuver traffic delay cost & $\left.+c_{p t} f_{d b} t_{s}\left(\lambda_{c}-\lambda_{c}^{*}\right)\right] \lambda_{p}$ \\
\hline On-street commercial vehicle parking & $\frac{c_{d p} \lambda_{p}}{\text { revenue }^{2}}$ \\
\hline On-street passenger car parking revenue & $\frac{c_{c o} \lambda_{c}^{*}}{\mu_{c}}$ \\
\hline
\end{tabular}

\section{Objective function}

$$
\begin{aligned}
y_{s o c}=\frac{c_{c o} \lambda_{c}^{*}}{\mu_{c}}+ & \frac{c_{p o} \lambda_{p}}{\mu_{p}}-c_{c t} \alpha_{w c}\left(\frac{\lambda_{c}^{*}}{n_{c} \mu_{c}}\right)^{\gamma_{c}} \lambda_{c} \\
& -\left[c_{p t}(1+\vartheta) \alpha_{w p}\left(\frac{\lambda_{p}}{n_{p} \mu_{p}}\right)^{\gamma_{p}}+c_{p t} f_{d b} t_{s}\left(\lambda_{c}-\lambda_{c}^{*}\right)\right] \lambda_{p}-c_{d c} \lambda_{c}^{*}-c_{d p} \lambda_{p} \\
& -c_{d b}\left(\lambda_{c}-\lambda_{c}^{*}\right)
\end{aligned}
$$




$$
\begin{array}{r}
y_{s o c}=\left[c_{c o}-c_{d c} \mu_{c}+c_{d b} \mu_{c}+c_{p t} f_{d b} t_{s} \lambda_{p} \mu_{c}\right] \frac{\lambda_{c}^{*}}{\mu_{c}}-c_{c t} \alpha_{w c}\left(\frac{\lambda_{c}^{*}}{n_{c} \mu_{c}}\right)^{\gamma_{c}} \lambda_{c}+\frac{c_{p o} \lambda_{p}}{\mu_{p}} \\
-c_{p t}(1+\vartheta) \alpha_{w p}\left(\frac{\lambda_{p}}{n_{p} \mu_{p}}\right)^{\gamma_{p}} \lambda_{p}-c_{d p} \lambda_{p}-c_{d b} \lambda_{c}-c_{p t} f_{d b} t_{s} \lambda_{c} \lambda_{p}
\end{array}
$$

Since $c_{c t} \alpha_{w c}\left(\frac{\lambda_{c}^{*}}{n_{c} \mu_{c}}\right)^{\gamma_{c}}=\frac{c_{v} n_{e}}{\mu_{c} \tau_{e}}-\frac{c_{c o}}{\mu_{c}}$,

$$
\begin{aligned}
& y_{s o c}=\left[c_{c o}-\right.\left.c_{d c} \mu_{c}+c_{d b} \mu_{c}+c_{p t} f_{d b} t_{s} \lambda_{p} \mu_{c}\right] \frac{\lambda_{c}^{*}}{\mu_{c}}-\left(\frac{c_{v} n_{e}}{\tau_{e}}-c_{c o}\right) \frac{\lambda_{c}}{\mu_{c}}+\frac{c_{p o} \lambda_{p}}{\mu_{p}} \\
&-c_{p t}(1+\vartheta) \alpha_{w p}\left(\frac{\lambda_{p}}{n_{p} \mu_{p}}\right)^{\gamma_{p}} \lambda_{p}-c_{d p} \lambda_{p}-c_{d b} \lambda_{c}-c_{p t} f_{d b} t_{s} \lambda_{c} \lambda_{p}
\end{aligned}
$$

Since $\lambda_{c}^{*}=n_{c} \mu_{c}\left(\frac{c_{v} n_{e}-c_{c o} \tau_{e}}{c_{c t} \alpha_{w c} \mu_{c} \tau_{e}}\right)^{\frac{1}{\gamma_{c}}}$

$$
\begin{aligned}
y_{s o c}=n_{c}\left[c_{c o}\right. & \left.-c_{d c} \mu_{c}+c_{d b} \mu_{c}+c_{p t} f_{d b} t_{s} \lambda_{p} \mu_{c}\right]\left(\frac{c_{v} n_{e}-c_{c o} \tau_{e}}{c_{c t} \alpha_{w c} \mu_{c} \tau_{e}}\right)^{\frac{1}{\gamma_{c}}}-\left(\frac{c_{v} n_{e}}{\tau_{e}}-c_{c o}\right) \frac{\lambda_{c}}{\mu_{c}} \\
& +\frac{c_{p o} \lambda_{p}}{\mu_{p}}-c_{p t}(1+\vartheta) \alpha_{w p}\left(\frac{\lambda_{p}}{n_{p} \mu_{p}}\right)^{\gamma_{p}} \lambda_{p}-c_{d p} \lambda_{p}-c_{d b} \lambda_{c} \\
& -c_{p t} f_{d b} t_{s} \lambda_{c} \lambda_{p}
\end{aligned}
$$




\section{Optimal $c_{\text {po }}$}

\section{$\underline{\text { Derivation of solution }}$}

The revenue is a linear function of $c_{p o}$. The constraint on $c_{p o}$ is,

$c_{p o} \leq c_{p f}+c_{p f f} \mu_{p}-c_{p t}(1+\vartheta) \alpha_{w p} \mu_{p}\left(\frac{\lambda_{p}}{n_{p} \mu_{p}}\right)^{\gamma_{p}}-c_{p t} \mu_{p} f_{d b} t_{s}\left(\lambda_{c}-\lambda_{c}^{*}\right)$

Since the problem is a linear programming, the optimal $c_{p o}$ is one of extreme points of the feasible region,

$c_{p o}^{*} \in\left\{0, c_{p f}+c_{p f f} \mu_{p}-c_{p t}(1+\vartheta) \alpha_{w p} \mu_{p}\left(\frac{\lambda_{p}}{n_{p} \mu_{p}}\right)^{\gamma_{p}}-c_{p t} \mu_{p} f_{d b} t_{s}\left(\lambda_{c}-\lambda_{c}^{*}\right)\right\}$

The revenue is an increasing function of $c_{p o}$. Therefore, the optimal solution is,

$c_{p o}^{*}=c_{p f}+c_{p f f} \mu_{p}-c_{p t}(1+\vartheta) \alpha_{w p} \mu_{p}\left(\frac{\lambda_{p}}{n_{p} \mu_{p}}\right)^{\gamma_{p}}-c_{p t} \mu_{p} f_{d b} t_{s}\left(\lambda_{c}-\lambda_{c}^{*}\right)$

\section{Optimal $c_{c o}$}

\section{Derivation of solution}

$$
\begin{aligned}
& \frac{\partial y_{s o c}}{\partial c_{c o}}=n_{c}\left(\frac{c_{v} n_{e}-c_{c o} \tau_{e}}{c_{c t} \alpha_{w c} \mu_{c} \tau_{e}}\right)^{\frac{1}{\gamma_{c}}}-\frac{n_{c}\left[c_{c o}-c_{d c} \mu_{c}+c_{d b} \mu_{c}+c_{p t} f_{d b} t_{s} \lambda_{p} \mu_{c}\right]}{\gamma_{c}\left(c_{c t} \alpha_{w c} \mu_{c}\right)^{\frac{1}{\gamma_{c}}}\left(\frac{c_{v} n_{e}}{\tau_{e}}-c_{c o}\right)^{\frac{\gamma_{c}-1}{\gamma_{c}}}}+\frac{\lambda_{c}}{\mu_{c}}=0 \\
& \frac{n_{c}\left[\gamma_{c} \frac{c_{v} n_{e}}{\tau_{e}}-\left(\gamma_{c}+1\right) c_{c o}+c_{d c} \mu_{c}-c_{d b} \mu_{c}-c_{p t} f_{d b} t_{s} \lambda_{p} \mu_{c}\right]}{\gamma_{c}\left(c_{c t} \alpha_{w c} \mu_{c}\right)^{\frac{1}{\gamma_{c}}}\left(\frac{c_{v} n_{e}}{\tau_{e}}-c_{c o}\right)^{\frac{\gamma_{c}-1}{\gamma_{c}}}}+\frac{\lambda_{c}}{\mu_{c}}=0 \\
& \begin{array}{l}
\text { If } \gamma_{c}=1, \\
\frac{n_{c}\left[\frac{c_{v} n_{e}}{\tau_{e}}-2 c_{c o}+c_{d c} \mu_{c}-c_{d b} \mu_{c}-c_{p t} f_{d b} t_{s} \lambda_{p} \mu_{c}\right]}{c_{c t} \alpha_{w c} \mu_{c}}+\frac{\lambda_{c}}{\mu_{c}}=0
\end{array}
\end{aligned}
$$


$\frac{c_{v} n_{e}}{\tau_{e}}-2 c_{c o}+c_{d c} \mu_{c}-c_{d b} \mu_{c}-c_{p t} f_{d b} t_{s} \lambda_{p} \mu_{c}+\frac{\lambda_{c} c_{c t} \alpha_{w c}}{n_{c}}=0$
$c_{c o}^{*}\left(\gamma_{c}=1\right)=\frac{c_{v} n_{e}}{2 \tau_{e}}+\frac{\left(c_{d c}-c_{d b}\right) \mu_{c}}{2}+\frac{\lambda_{c} c_{c t} \alpha_{w c}}{2 n_{c}}-\frac{c_{p t} f_{d b} t_{s} \lambda_{p} \mu_{c}}{2}$

$\underline{\text { Optimality condition }}$

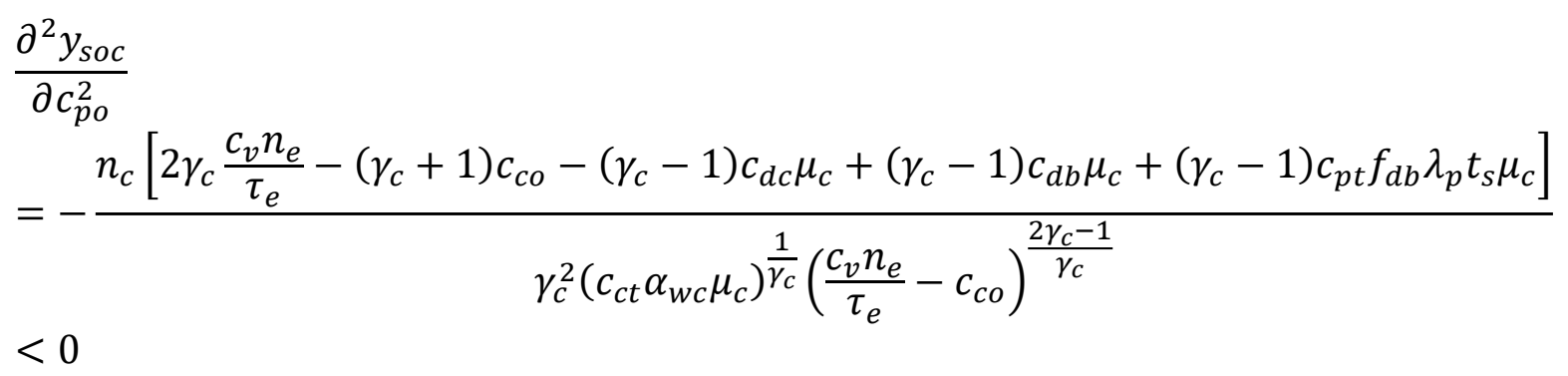

The solution is a local maximum.

\section{Optimal $\boldsymbol{n}_{\boldsymbol{p}}$}

Let $\gamma_{c}=\gamma_{p}=1$,

Since $n_{c}=\frac{l_{b}-n_{p} l_{p}}{l_{c}}$,

$$
\begin{aligned}
y_{s o c}=\frac{1}{c_{c t} \alpha_{w c} \mu_{c}} & \left(\frac{l_{b}-n_{p} l_{p}}{l_{c}}\right)\left(c_{c o}-c_{d c} \mu_{c}+c_{d b} \mu_{c}+c_{p t} f_{d b} t_{s} \lambda_{p} \mu_{c}\right)\left(\frac{c_{v} n_{e}}{\tau_{e}}-c_{c o}\right) \\
& -\left(\frac{c_{v} n_{e}}{\tau_{e}}-c_{c o}\right) \frac{\lambda_{c}}{\mu_{c}}+\frac{c_{p o} \lambda_{p}}{\mu_{p}}-c_{p t}(1+\vartheta) \alpha_{w p}\left(\frac{\lambda_{p}}{n_{p} \mu_{p}}\right) \lambda_{p}-c_{d p} \lambda_{p} \\
& -c_{d b} \lambda_{c}-c_{p t} f_{d b} t_{s} \lambda_{c} \lambda_{p}
\end{aligned}
$$

$$
\begin{gathered}
\frac{\partial y_{s o c}}{\partial n_{p}}=-\frac{1}{c_{c t} \alpha_{w c} \mu_{c}}\left(\frac{l_{p}}{l_{c}}\right)\left(c_{c o}-c_{d c} \mu_{c}+c_{d b} \mu_{c}+c_{p t} f_{d b} t_{s} \lambda_{p} \mu_{c}\right)\left(\frac{c_{v} n_{e}}{\tau_{e}}-c_{c o}\right) \\
+\frac{c_{p t}(1+\vartheta) \alpha_{w p} \lambda_{p}^{2}}{n_{p}^{2} \mu_{p}}=0
\end{gathered}
$$




$$
\begin{aligned}
& \frac{c_{p t}(1+\vartheta) \alpha_{w p} \lambda_{p}^{2}}{n_{p}^{2} \mu_{p}}=\frac{1}{c_{c t} \alpha_{w c} \mu_{c}}\left(\frac{l_{p}}{l_{c}}\right)\left(c_{c o}-c_{d c} \mu_{c}+c_{d b} \mu_{c}+c_{p t} f_{d b} t_{s} \lambda_{p} \mu_{c}\right)\left(\frac{c_{v} n_{e}}{\tau_{e}}-c_{c o}\right) \\
& n_{p}^{*}\left(\gamma_{c}=\gamma_{p}=1\right) \\
& =\lambda_{p} \sqrt{\frac{c_{c t} \alpha_{w c} c_{p t}(1+\vartheta) \alpha_{w p}}{\left(\frac{c_{v} n_{e}}{\tau_{e}}-c_{c o}\right)\left(c_{c o}-c_{d c} \mu_{c}+c_{d b} \mu_{c}+c_{p t} f_{d b} t_{s} \lambda_{p} \mu_{c}\right)}\left(\frac{\mu_{c}}{\mu_{p}}\right)\left(\frac{l_{c}}{l_{p}}\right)}
\end{aligned}
$$

Optimality condition

$\frac{\partial^{2} y_{n e t}}{\partial n_{p}^{2}}=-\frac{2 c_{p t}(1+\vartheta) \alpha_{w p} \lambda_{p}^{2}}{n_{p}^{3} \mu_{p}}<0$

The solution is a local maximum. 


\section{$\underline{\text { Social optimum (SOC) model (scenario 4): formulation and derivation }}$}

\section{Scenario Information}

$\lambda_{c}^{*}<\lambda_{c}$

Some commercial vehicles double parking

$\lambda_{p}^{*}<\lambda_{p}$

Some cars parking off street

\section{Cost of commercial vehicles parking}

$\underline{\text { Per-stop monetary cost of commercial vehicles parking on street }}$

$\frac{c_{c o}}{\mu_{c}}$

$\underline{\text { Per-stop waiting time of commercial vehicles parking on street }}$

$\alpha_{w c}\left(\frac{\lambda_{c}^{*}}{n_{c} \mu_{c}}\right)^{\gamma_{c}} \quad \gamma_{c} \geq 1$

Per-stop parking search cost of commercial vehicles parking on street

Assumption: The time constraint is binding.

$c_{c t} \alpha_{w c}\left(\frac{\lambda_{c}^{*}}{n_{c} \mu_{c}}\right)^{\gamma_{c}}$

Per-stop double parking cost of commercial vehicles

$\frac{c_{v} n_{e}}{\mu_{c} \tau_{e}}$

Breakeven demand for "paying on-street" commercial parking: $\lambda_{c}^{*}$

(no impact of passengers on commercial)

$$
\begin{aligned}
& \frac{c_{c o}}{\mu_{c}}+c_{c t} \alpha_{w c}\left(\frac{\lambda_{c}^{*}}{n_{c} \mu_{c}}\right)^{\gamma_{c}}=\frac{c_{v} n_{e}}{\mu_{c} \tau_{e}} \\
& \left(\frac{\lambda_{c}^{*}}{n_{c} \mu_{c}}\right)^{\gamma_{c}}=\frac{c_{v} n_{e}-c_{c o} \tau_{e}}{c_{c t} \alpha_{w c} \mu_{c} \tau_{e}} \\
& \lambda_{c}^{*}=\frac{n_{c} \mu_{c}}{\left(c_{c t} \alpha_{w c} \mu_{c}\right)^{\frac{1}{\gamma_{c}}}}\left(\frac{c_{v} n_{e}}{\tau_{e}}-c_{c o}\right)^{\frac{1}{\gamma_{c}}}
\end{aligned}
$$




\section{Cost of passenger cars parking}

Per-stop monetary cost of a passenger car parking on street

$\frac{c_{p o}}{\mu_{p}}$

Per-stop parking waiting time of a passenger car

$\alpha_{w p}\left(\frac{\lambda_{p}^{*}}{n_{p} \mu_{p}}\right)^{\gamma_{p}} \quad \gamma_{p} \geq \gamma_{c} \geq 1$

Per-stop parking search cost of a passenger car

Assumption: The time constraint is binding.

$c_{p t}(1+\vartheta) \alpha_{w p}\left(\frac{\lambda_{p}^{*}}{n_{p} \mu_{p}}\right)^{\gamma_{p}}$

Expected number of double-parking events that a passenger car will find while searching

$t_{s}\left(\lambda_{c}-\lambda_{c}^{*}\right)$

$\underline{\text { Per-stop delay cost of a passenger car in queue caused by double-parking commercial }}$

$\underline{\text { vehicles }}$

$c_{p t} f_{d b} t_{s}\left(\lambda_{c}-\lambda_{c}^{*}\right)$

Per-stop parking cost of a passenger car parking off street

$\frac{c_{p f}}{\mu_{p}}+c_{p f f}$

Breakeven demand for "paying on-street" cars parking: $\lambda_{p}^{*}$

$$
\begin{aligned}
& \frac{c_{p o}}{\mu_{p}}+c_{p t}(1+\vartheta) \alpha_{w p}\left(\frac{\lambda_{p}^{*}}{n_{p} \mu_{p}}\right)^{\gamma_{p}}+c_{p t} f_{d b} t_{s}\left(\lambda_{c}-\lambda_{c}^{*}\right)=\frac{c_{p f}}{\mu_{p}}+c_{p f f} \\
& c_{p t}(1+\vartheta) \alpha_{w p}\left(\frac{\lambda_{p}^{*}}{n_{p} \mu_{p}}\right)^{\gamma_{p}}=\frac{c_{p f}}{\mu_{p}}+c_{p f f}-\frac{c_{p o}}{\mu_{p}}-c_{p t} f_{d b} t_{s}\left(\lambda_{c}-\lambda_{c}^{*}\right) \\
& \lambda_{p}^{*}=\frac{n_{p} \mu_{p}}{\left(c_{p t}(1+\vartheta) \alpha_{w p} \mu_{p}\right)^{\frac{1}{\gamma_{p}}}}\left[c_{p f}+c_{p f f} \mu_{p}-c_{p o}-c_{p t} f_{d b} t_{s} \mu_{p}\left(\lambda_{c}-\lambda_{c}^{*}\right)\right]^{\frac{1}{\gamma_{p}}} \\
& \text { Since } \lambda_{c}^{*}=\frac{n_{c} \mu_{c}}{\left(c_{c t} \alpha_{w c} \mu_{c}\right)^{\frac{1}{\gamma_{c}}}}\left(\frac{c_{v} n_{e}}{\tau_{e}}-c_{c o}\right)^{\frac{1}{\gamma_{c}}}
\end{aligned}
$$




$$
\begin{gathered}
\lambda_{p}^{*}=\frac{n_{p} \mu_{p}}{\left(c_{p t}(1+\vartheta) \alpha_{w p} \mu_{p}\right)^{\frac{1}{\gamma_{p}}}}\left[c_{p f}+c_{p f f} \mu_{p}-c_{p o}\right. \\
\left.-c_{p t} f_{d b} t_{s} \mu_{p}\left\{\lambda_{c}-n_{c} \mu_{c}\left(\frac{c_{v} n_{e}-c_{c o} \tau_{e}}{c_{c t} \alpha_{w c} \mu_{c} \tau_{e}}\right)^{\frac{1}{\gamma_{c}}}\right)\right]^{\frac{1}{\gamma_{p}}}
\end{gathered}
$$

Summary of the relevant revenue and cost (unit: dollars per hour)

\begin{tabular}{|l|c|}
\hline Components & Formula \\
\hline Commercial parking delay cost & $c_{c t} \alpha_{w c}\left(\frac{\lambda_{c}^{*}}{n_{c} \mu_{c}}\right)^{\gamma_{c}} \lambda_{c}$ \\
\hline Commercial maneuver traffic delay & $c_{d c} \lambda_{c}^{*}$ \\
\hline Double parking delay cost & $c_{d b}\left(\lambda_{c}-\lambda_{c}^{*}\right)$ \\
\hline Car parking delay cost & {$\left[c_{p t}(1+\vartheta) \alpha_{w p}\left(\frac{\lambda_{p}^{*}}{n_{p} \mu_{p}}\right)^{\gamma_{p}}\right.$} \\
\hline revenue & $\left.+c_{p t} f_{d b} t_{s}\left(\lambda_{c}-\lambda_{c}^{*}\right)\right] \lambda_{p}$ \\
\hline parking revenue & $\frac{c_{d p} \lambda_{p}^{*}}{\mu_{p}}$ \\
\hline On-street passenger car parking & $\frac{c_{c o} \lambda_{c}^{*}}{\mu_{c}}$ \\
\hline On-street commercial vehicle & $\frac{c_{p o} \lambda_{p}^{*}}{\mu_{p}}$ \\
\hline
\end{tabular}




\section{Objective function}

$$
\begin{aligned}
y_{s o c}=\frac{c_{c o} \lambda_{c}^{*}}{\mu_{c}}+ & \frac{c_{p o} \lambda_{p}^{*}}{\mu_{p}}-c_{c t} \alpha_{w c}\left(\frac{\lambda_{c}^{*}}{n_{c} \mu_{c}}\right)^{\gamma_{c}} \lambda_{c} \\
& -\left[c_{p t}(1+\vartheta) \alpha_{w p}\left(\frac{\lambda_{p}^{*}}{n_{p} \mu_{p}}\right)^{\gamma_{p}}+c_{p t} f_{d b} t_{s}\left(\lambda_{c}-\lambda_{c}^{*}\right)\right] \lambda_{p}-c_{d c} \lambda_{c}^{*}-c_{d p} \lambda_{p}^{*} \\
& -c_{d b}\left(\lambda_{c}-\lambda_{c}^{*}\right) \\
y_{s o c}=\left(c_{c o}-\right. & \left.c_{d c} \mu_{c}+c_{d b} \mu_{c}\right) \frac{\lambda_{c}^{*}}{\mu_{c}}-c_{c t} \alpha_{w c}\left(\frac{\lambda_{c}^{*}}{n_{c} \mu_{c}}\right)^{\gamma_{c}} \lambda_{c}+\left(c_{p o}-c_{d p} \mu_{p}\right) \frac{\lambda_{p}^{*}}{\mu_{p}} \\
& -\left[c_{p t}(1+\vartheta) \alpha_{w p}\left(\frac{\lambda_{p}^{*}}{n_{p} \mu_{p}}\right)^{\gamma_{p}}+c_{p t} f_{d b} t_{s}\left(\lambda_{c}-\lambda_{c}^{*}\right)\right] \lambda_{p}-c_{d b} \lambda_{c}
\end{aligned}
$$

Since $c_{c t} \alpha_{w c}\left(\frac{\lambda_{c}^{*}}{n_{c} \mu_{c}}\right)^{\gamma_{c}}=\frac{c_{v} n_{e}}{\mu_{c} \tau_{e}}-\frac{c_{c o}}{\mu_{c}}$

Since $c_{p t}(1+\vartheta) \alpha_{w p}\left(\frac{\lambda_{p}^{*}}{n_{p} \mu_{p}}\right)^{\gamma_{p}}+c_{p t} f_{d b} t_{s}\left(\lambda_{c}-\lambda_{c}^{*}\right)=\frac{c_{p f}}{\mu_{p}}+c_{p f f}-\frac{c_{p o}}{\mu_{p}}$

$$
\begin{gathered}
y_{s o c}=\left(c_{c o}-c_{d c} \mu_{c}+c_{d b} \mu_{c}\right) \frac{\lambda_{c}^{*}}{\mu_{c}}-\left(\frac{c_{v} n_{e}}{\tau_{e}}-c_{c o}\right) \frac{\lambda_{c}}{\mu_{c}}+\left(c_{p o}-c_{d p} \mu_{p}\right) \frac{\lambda_{p}^{*}}{\mu_{p}} \\
-\left(c_{p f}+c_{p f f} \mu_{p}-c_{p o}\right) \frac{\lambda_{p}}{\mu_{p}}-c_{d b} \lambda_{c}
\end{gathered}
$$

Since $\lambda_{c}^{*}=\frac{n_{c} \mu_{c}}{\left(c_{c t} \alpha_{w c} \mu_{c}\right)^{\frac{1}{\gamma_{c}}}}\left(\frac{c_{v} n_{e}}{\tau_{e}}-c_{c o}\right)^{\frac{1}{\gamma_{c}}}$ 


$$
\begin{aligned}
& \text { Since } \lambda_{p}^{*}=\frac{n_{p} \mu_{p}}{\left(c_{p t}(1+\vartheta) \alpha_{w p} \mu_{p}\right)^{\frac{1}{\gamma_{p}}}}\left[c_{p f}+c_{p f f} \mu_{p}-c_{p o}\right. \\
& \left.-c_{p t} f_{d b} t_{s} \mu_{p}\left\{\lambda_{c}-n_{c} \mu_{c}\left(\frac{c_{v} n_{e}-c_{c o} \tau_{e}}{c_{c t} \alpha_{w c} \mu_{c} \tau_{e}}\right)^{\frac{1}{\gamma_{c}}}\right\}\right]^{\frac{1}{\gamma_{p}}} \\
& y_{s o c}=\frac{n_{c}\left(c_{c o}-c_{d c} \mu_{c}+c_{d b} \mu_{c}\right)}{\left(c_{c t} \alpha_{w c} \mu_{c}\right)^{\frac{1}{\gamma_{c}}}}\left(\frac{c_{v} n_{e}}{\tau_{e}}-c_{c o}\right)^{\frac{1}{\gamma_{c}}}-\left(\frac{c_{v} n_{e}}{\tau_{e}}-c_{c o}\right) \frac{\lambda_{c}}{\mu_{c}} \\
& -\left(c_{p f}+c_{p f f} \mu_{p}-c_{p o}\right) \frac{\lambda_{p}}{\mu_{p}}-c_{d b} \lambda_{c} \\
& +\frac{n_{p}\left(c_{p o}-c_{d p} \mu_{p}\right)}{\left(c_{p t}(1+\vartheta) \alpha_{w p} \mu_{p}\right)^{\frac{1}{\gamma_{p}}}}\left[c_{p f}+c_{p f f} \mu_{p}-c_{p o}\right. \\
& \left.-c_{p t} f_{d b} t_{s} \mu_{p}\left\{\lambda_{c}-n_{c} \mu_{c}\left(\frac{c_{v} n_{e}-c_{c o} \tau_{e}}{c_{c t} \alpha_{w c} \mu_{c} \tau_{e}}\right)^{\frac{1}{\gamma_{c}}}\right\}\right]^{\frac{1}{\gamma_{p}}} \\
& \text { Let } A=\left(c_{c t} \alpha_{w c} \mu_{c}\right)^{\frac{1}{\gamma_{c}}} \\
& \text { Let } B=\left(c_{p t}(1+\vartheta) \alpha_{w p} \mu_{p}\right)^{\frac{1}{\gamma_{p}}} \\
& \text { Let } D=c_{p f}+c_{p f f} \mu_{p}-c_{p o}-c_{p t} f_{d b} t_{s} \mu_{p}\left\{\lambda_{c}-n_{c} \mu_{c}\left(\frac{c_{v} n_{e}-c_{c o} \tau_{e}}{c_{c t} \alpha_{w c} \mu_{c} \tau_{e}}\right)^{\frac{1}{\gamma_{c}}}\right\}
\end{aligned}
$$


Since $A=\left(c_{c t} \alpha_{w c} \mu_{c}\right)^{\frac{1}{\gamma_{c}}}$

$D=c_{p f}+c_{p f f} \mu_{p}-c_{p o}-c_{p t} f_{d b} t_{s} \mu_{p}\left\{\lambda_{c}-\frac{n_{c} \mu_{c}}{A}\left(\frac{c_{v} n_{e}}{\tau_{e}}-c_{c o}\right)^{\frac{1}{\gamma_{c}}}\right\}$

$\frac{\partial D}{\partial c_{p o}}=-1$

$\frac{\partial D}{\partial c_{c o}}=-\frac{c_{p t} f_{d b} t_{s} \mu_{p} n_{c} \mu_{c}}{\gamma_{c} A\left(\frac{c_{v} n_{e}}{\tau_{e}}-c_{c o}\right)^{\frac{\gamma_{c}-1}{\gamma_{c}}}}$

$y_{s o c}=\frac{n_{c}\left(c_{c o}-c_{d c} \mu_{c}+c_{d b} \mu_{c}\right)}{A}\left(\frac{c_{v} n_{e}}{\tau_{e}}-c_{c o}\right)^{\frac{1}{\gamma_{c}}}-\left(\frac{c_{v} n_{e}}{\tau_{e}}-c_{c o}\right) \frac{\lambda_{c}}{\mu_{c}}$

$-\left(c_{p f}+c_{p f f} \mu_{p}-c_{p o}\right) \frac{\lambda_{p}}{\mu_{p}}-c_{d b} \lambda_{c}+\frac{n_{p}\left(c_{p o}-c_{d p} \mu_{p}\right)}{B} D^{\frac{1}{\gamma_{p}}}$

\section{Optimal $c_{\text {po }}$}

$$
\begin{aligned}
& \frac{\partial y_{s o c}}{\partial c_{p o}}=\frac{n_{p}}{B} D^{\frac{1}{\gamma_{p}}}-\frac{n_{p}\left(c_{p o}-c_{d p} \mu_{p}\right)}{\gamma_{p} B}\left(D^{\frac{1-\gamma_{p}}{\gamma_{p}}}\right)+\frac{\lambda_{p}}{\mu_{p}}=0 \\
& \frac{n_{p}}{B} D^{\frac{1}{\gamma_{p}}}-\frac{n_{p}\left(c_{p o}-c_{d p} \mu_{p}\right)}{\gamma_{p} B}\left(D^{\frac{1-\gamma_{p}}{\gamma_{p}}}\right)+\frac{\lambda_{p}}{\mu_{p}}=0 \\
& \text { If } \gamma_{p}=1, \\
& \frac{n_{p}}{B} D-\frac{n_{p}\left(c_{p o}-c_{d p} \mu_{p}\right)}{B}+\frac{\lambda_{p}}{\mu_{p}}=0
\end{aligned}
$$




$$
c_{p o}=D+c_{d p} \mu_{p}+\frac{\lambda_{p} B}{n_{p} \mu_{p}}
$$

Since $D\left(\gamma_{c}=1\right)=c_{p f}+c_{p f f} \mu_{p}-c_{p o}-c_{p t} f_{d b} t_{s} \mu_{p}\left\{\lambda_{c}-n_{c} \mu_{c}\left(\frac{c_{v} n_{e}-c_{c o} \tau_{e}}{c_{c t} \alpha_{w c} \mu_{c} \tau_{e}}\right)\right\}$

$$
\begin{aligned}
c_{p o}^{*}=\frac{\left(c_{p f}+c_{p f f} \mu_{p}\right)}{2}+\frac{c_{d p} \mu_{p}}{2}+\frac{\lambda_{p} c_{p t}(1+\vartheta) \alpha_{w p}}{2 n_{p}} \\
-\frac{c_{p t} f_{d b} t_{s} \mu_{p}}{2}\left[\lambda_{c}-n_{c} \mu_{c}\left(\frac{c_{v} n_{e}-c_{c o} \tau_{e}}{c_{c t} \alpha_{w c} \mu_{c} \tau_{e}}\right)\right]
\end{aligned}
$$

$$
\begin{gathered}
\text { Since } \lambda_{c}^{*}=\frac{n_{c} \mu_{c}}{c_{c t} \alpha_{w c} \mu_{c}}\left(\frac{c_{v} n_{e}}{\tau_{e}}-c_{c o}\right) \\
\begin{aligned}
c_{p o}^{*}\left(\gamma_{p}=1\right)= & \frac{\left(c_{p f}+c_{p f f} \mu_{p}\right)}{2}+\frac{c_{d p} \mu_{p}}{2}+\frac{\lambda_{p} c_{p t}(1+\vartheta) \alpha_{w p}}{2 n_{p}} \\
& -\frac{c_{p t} f_{d b} t_{s} \mu_{p}}{2}\left(\lambda_{c}-\lambda_{c}^{*}\right)(H .5)
\end{aligned}
\end{gathered}
$$

Optimality condition

$$
\frac{\partial^{2} y_{s o c}\left(\gamma_{c}=\gamma_{p}=1\right)}{\partial c_{p o}^{2}}=-\frac{2 n_{p}}{c_{p t}(1+\vartheta) \alpha_{w p} \mu_{p}}<0
$$

The solution is a local maximum. 


\section{Optimal $c_{c o}$}

$$
\begin{aligned}
& \frac{\partial y_{s o c}}{\partial c_{c o}}=0 \\
& \frac{n_{c}}{A}\left(\frac{c_{v} n_{e}}{\tau_{e}}-c_{c o}\right)^{\frac{1}{\gamma_{c}}}-\frac{n_{c}\left(c_{c o}-c_{d c} \mu_{c}+c_{d b} \mu_{c}\right)}{\gamma_{c} A\left(\frac{c_{v} n_{e}}{\tau_{e}}-c_{c o}\right)^{\frac{\gamma_{c}-1}{\gamma_{c}}}}-\frac{c_{p t} f_{d b} t_{s} n_{c} \mu_{c} n_{p} \mu_{p}\left(c_{p o}-c_{d p} \mu_{p}\right)}{\gamma_{c} \gamma_{p} A B D^{\frac{\gamma_{p}-1}{\gamma_{p}}}\left(\frac{c_{v} n_{e}}{\tau_{e}}-c_{c o}\right)^{\frac{\gamma_{c}-1}{\gamma_{c}}}}+\frac{\lambda_{c}}{\mu_{c}} \\
& =0 \quad(H .6) \\
& \text { If } \gamma_{c}=\gamma_{p}=1 \\
& \frac{n_{c}}{c_{c t} \alpha_{w c} \mu_{c}}\left(\frac{c_{v} n_{e}}{\tau_{e}}-c_{c o}\right)-\frac{n_{c}\left(c_{c o}-c_{d c} \mu_{c}+c_{d b} \mu_{c}\right)}{c_{c t} \alpha_{w c} \mu_{c}}-\frac{c_{p t} f_{d b} t_{s} n_{c} \mu_{c} n_{p} \mu_{p}\left(c_{p o}-c_{d p} \mu_{p}\right)}{\left(c_{c t} \alpha_{w c} \mu_{c}\right)\left[c_{p t}(1+\vartheta) \alpha_{w p} \mu_{p}\right]} \\
& +\frac{\lambda_{c}}{\mu_{c}}=0 \\
& \frac{c_{v} n_{e}}{\tau_{e}}-c_{c o}-c_{c o}+\left(c_{d c}-c_{d b}\right) \mu_{c}-\frac{f_{d b} t_{s} \mu_{c} n_{p}\left(c_{p o}-c_{d p} \mu_{p}\right)}{(1+\vartheta) \alpha_{w p}}+\frac{\lambda_{c} c_{c t} \alpha_{w c}}{n_{c}}=0 \\
& c_{c o}^{*}\left(\gamma_{c}=\gamma_{p}=1\right) \\
& =\frac{c_{v} n_{e}}{2 \tau_{e}}+\frac{\left(c_{d c}-c_{d b}\right) \mu_{c}}{2}+\frac{\lambda_{c} c_{c t} \alpha_{w c}}{2 n_{c}} \\
& -\frac{f_{d b} t_{s} \mu_{c} n_{p}\left(c_{p o}-c_{d p} \mu_{p}\right)}{2(1+\vartheta) \alpha_{w p}} \quad(H .7)
\end{aligned}
$$

Optimality condition

$$
\frac{\partial^{2} y_{s o c}\left(\gamma_{c}=\gamma_{p}=1\right)}{\partial c_{c o}^{2}}=-\frac{2}{c_{c t} \alpha_{w c} \mu_{c}}\left(\frac{l_{b}-n_{p} l_{p}}{l_{c}}\right)<0
$$

The solution is a local maximum. 


\section{Optimal $\boldsymbol{n}_{\boldsymbol{p}}$}

Let $\gamma_{c}=\gamma_{p}=1$,

Since $n_{c}=\frac{l_{b}-n_{p} l_{p}}{l_{c}}$,

$$
\begin{array}{r}
y_{s o c}=\frac{\left(c_{c o}-c_{d c} \mu_{c}+c_{d b} \mu_{c}\right)}{A}\left(\frac{l_{b}-n_{p} l_{p}}{l_{c}}\right)\left(\frac{c_{v} n_{e}}{\tau_{e}}-c_{c o}\right)-\left(\frac{c_{v} n_{e}}{\tau_{e}}-c_{c o}\right) \frac{\lambda_{c}}{\mu_{c}} \\
-\left(c_{p f}+c_{p f f} \mu_{p}-c_{p o}\right) \frac{\lambda_{p}}{\mu_{p}}-c_{d b} \lambda_{c}+\frac{n_{p}\left(c_{p o}-c_{d p} \mu_{p}\right) D}{B}
\end{array}
$$

$$
\begin{aligned}
\frac{\partial y_{s o c}}{\partial n_{p}}=\left(\frac{-l_{p}}{l_{c}}\right) & \frac{\left(c_{c o}-c_{d c} \mu_{c}+c_{d b} \mu_{c}\right)}{A}\left(\frac{c_{v} n_{e}}{\tau_{e}}-c_{c o}\right) \\
& +\frac{\left(c_{p o}-c_{d p} \mu_{p}\right)}{B}\left[D-n_{p} c_{p t} f_{d b} t_{s} \mu_{p} \mu_{c}\left(\frac{c_{v} n_{e}-c_{c o} \tau_{e}}{c_{c t} \alpha_{w c} \mu_{c} \tau_{e}}\right)\left(\frac{l_{p}}{l_{c}}\right)\right]=0
\end{aligned}
$$

$$
\begin{gathered}
\text { Since } D\left(\gamma_{c}=1\right)=c_{p f}+c_{p f f} \mu_{p}-c_{p o}-c_{p t} f_{d b} t_{s} \mu_{p}\left\{\lambda_{c}-n_{c} \mu_{c}\left(\frac{c_{v} n_{e}-c_{c o} \tau_{e}}{c_{c t} \alpha_{w c} \mu_{c} \tau_{e}}\right)\right\} \\
c_{p f}+c_{p f f} \mu_{p}-c_{p o}-c_{p t} f_{d b} t_{s} \mu_{p}\left\{\lambda_{c}+\left(\frac{-l_{b}+2 n_{p} l_{p}}{l_{c}}\right) \mu_{c}\left(\frac{c_{v} n_{e}-c_{c o} \tau_{e}}{c_{c t} \alpha_{w c} \mu_{c} \tau_{e}}\right)\right\} \\
=\left(\frac{l_{p}}{l_{c}}\right) \frac{B\left(c_{c o}-c_{d c} \mu_{c}+c_{d b} \mu_{c}\right)}{A\left(c_{p o}-c_{d p} \mu_{p}\right)}\left(\frac{c_{v} n_{e}}{\tau_{e}}-c_{c o}\right)
\end{gathered}
$$




$$
\begin{aligned}
& n_{p}^{*}\left(\gamma_{c}=\gamma_{p}=1\right)= \frac{l_{b}}{2 l_{p}}-\lambda_{c} \alpha_{w c}\left(\frac{l_{c}}{2 l_{p}}\right)\left(\frac{c_{c t}}{\frac{c_{v} n_{e}}{\tau_{e}}-c_{c o}}\right) \\
&+\frac{1}{2 f_{d b} t_{s} \mu_{p}}\left[\alpha_{w c}\left(\frac{l_{c}}{l_{p}}\right)\left(\frac{c_{c t}}{c_{p t}}\right)\left(\frac{c_{p f}+c_{p f f} \mu_{p}-c_{p o}}{\frac{c_{v} n_{e}}{\tau_{e}}-c_{c o}}\right)\right. \\
&\left.-(1+\vartheta) \alpha_{w p}\left(\frac{\mu_{p}}{\mu_{c}}\right)\left(\frac{c_{c o}-c_{d c} \mu_{c}+c_{d b} \mu_{c}}{c_{p o}-c_{d p} \mu_{p}}\right)\right]
\end{aligned}
$$

\section{Optimality condition}

$$
\frac{\partial^{2} y_{s o c}\left(\gamma_{c}=\gamma_{p}=1\right)}{\partial n_{p}^{2}}=-\left(\frac{2 l_{p}}{l_{c}}\right)\left(c_{p o}-c_{d p} \mu_{p}\right)\left(\frac{c_{v} n_{e}}{\tau_{e}}-c_{c o}\right) \frac{f_{d b} t_{s}}{(1+\vartheta) \alpha_{w p} c_{c t} \alpha_{w c}}<0
$$

The solution is a local maximum. 


\title{
Appendix B: R code for corridor traffic delay simulation
}

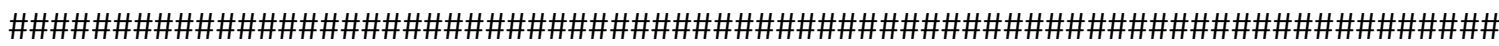 \\ \#\#\# Input

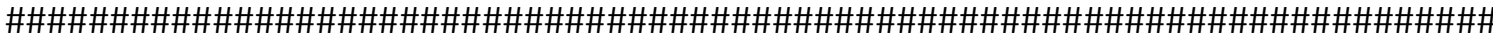 \\ \# Distance of upstream and downstream intersections (unit = feet) \\ D2 $<-450 \quad$ \# entry intersection \\ D3 <- $700 \quad$ \# intersection upstream of double parking \\ D4 $<-1000 \quad$ \# intersection downstream of double parking \\ \# Traffic \\ $\mathrm{NL}<-2 \quad$ \# number of lanes \\ Va $<-1600 \quad$ \# traffic demand (vph) \\ $\mathrm{Vc}<-1348 * \mathrm{NL} \quad$ \# roadway capacity \\ $\mathrm{Fb}<-0.40 \quad$ \# flow rate reduction factor due to double parking \\ $\mathrm{Vb}<-\mathrm{Fb} * \mathrm{Vc}$ \\ $\mathrm{S}<-28 \quad$ \# vehicle speed at an uncongested flow for urban streets (mph) \\ $\mathrm{Kj}<-0.200 \quad$ \# jam density (vehicle/meter/lane) \\ \# Signal cycle time (second) \\ red2 $<-20 \quad$ \# red time interval of entry intersection \\ red3 $<-20 \quad$ \# red time interval of upstream intersection \\ red4 $<-20 \quad$ \# red time interval of downstream intersection \\ grn2 $<-40 \quad$ \# green time interval of entry intersection \\ grn3 $<-40 \quad$ \# green time interval of upstream intersection \\ grn4 $<-40 \quad$ \# green time interval of downstream intersection \\ \# Location of a double-parked vehicle \\ QL_max_4th <- Vc * red4 / Kj \# Max queue length at downstream intersection \\ Ddp $<-800 \quad$ \# Double-parked vehicle location \\ \# Start time of signals \\ red2_st $<-1$ \# when first red light of entry intersection starts \\ red3_st $<-1$ \# when first red light of upstream intersection starts \\ red4_st $<-1 \quad$ \# when first red light of downstream intersection starts \\ \# Double-parking duration \\ Dp_st <-red3_st+3*(red3+grn3)+red3 \# starting time to double park (second) \\ Dp_dr $<-3 * 60$ \# double parking duration (second) \\ \# Change units of parameters to feet, second, and density per 2 lanes
}




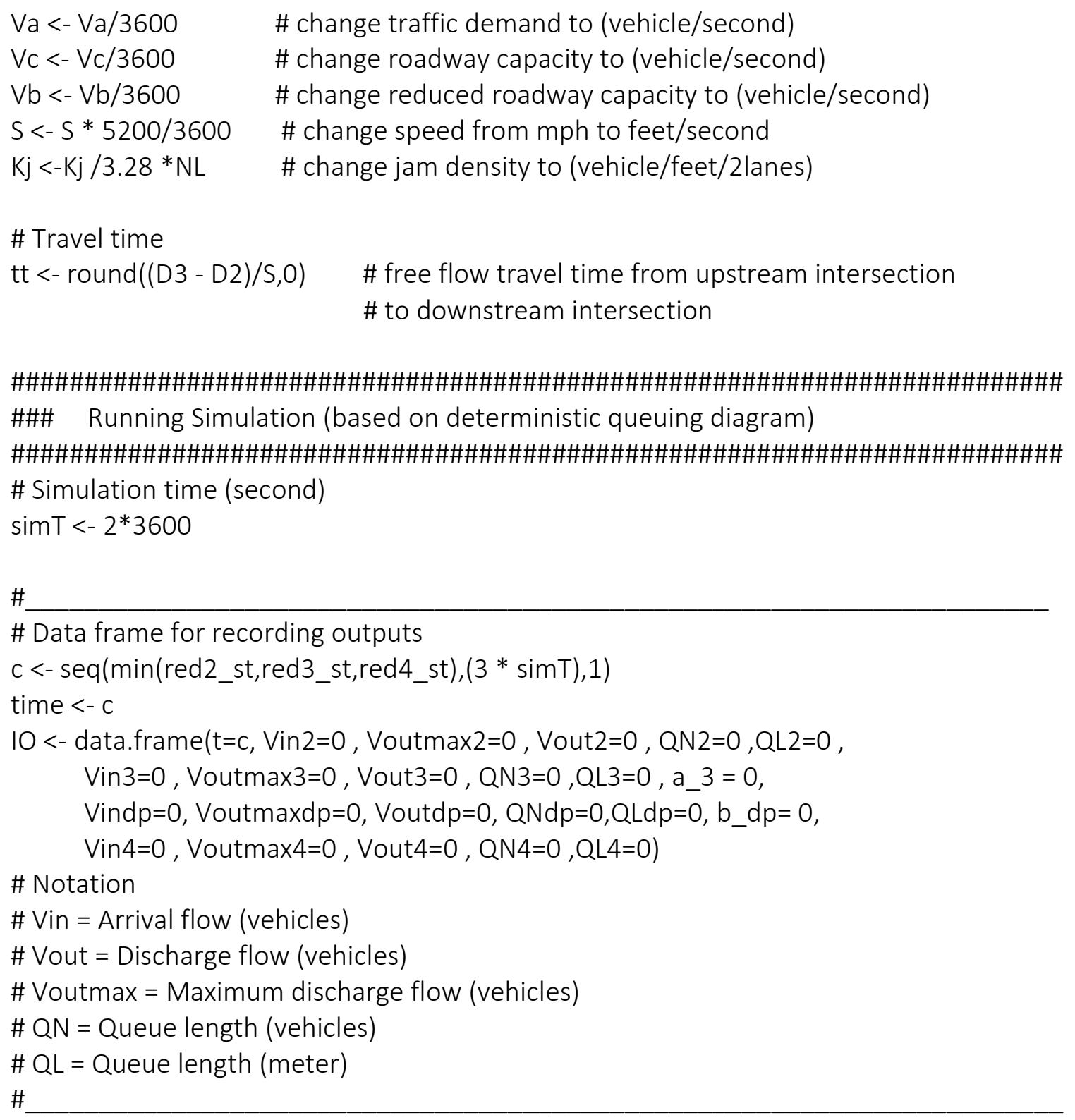

\# Set maximum discharging flows at entry intersection

$\mathrm{t}<-$ red2_st

while $(t<\operatorname{sim} T)\{$

$\mathrm{t}<-\mathrm{t}+\mathrm{red} 2$

for (grn in 1:grn2) \{

IO\$Voutmax2[which(time==t+grn)] $<-1$

\}

$t<-t+g r n 2$

\}

IO\$Voutmax2 <- IO\$Voutmax2 * Vc 


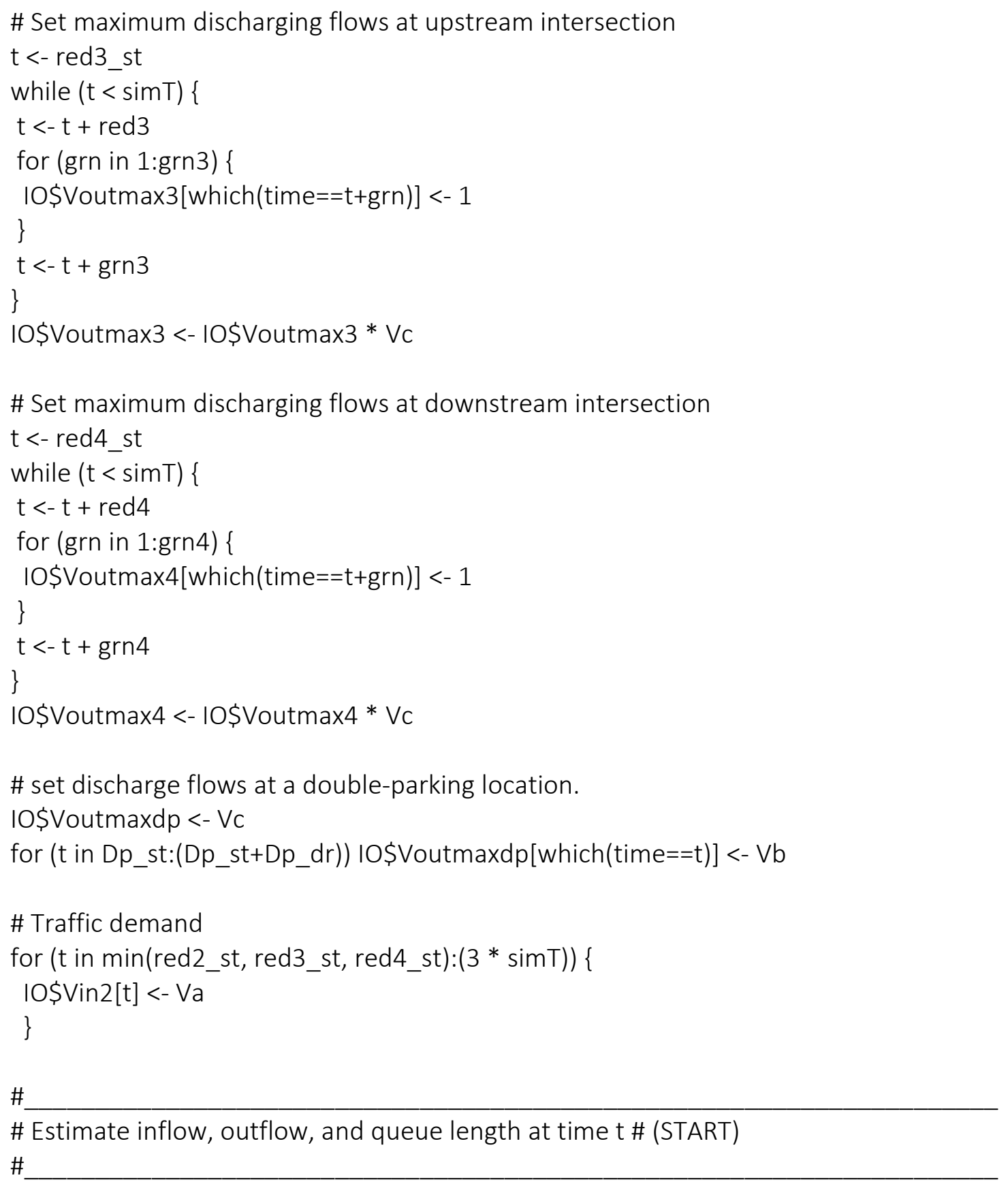

\# starting time

tmin $<-$ min (red2_st, red3_st, red4_st) +1

\# Parameters to check queue spill back

$a<-0 \quad \#(a=2)$ means queue spilling over the entry intersection

$\mathrm{b}<-0 \quad \#(b=1)$ means queue spilling over the upstream intersection 
\#\# Process of estimating flows and queue at time $t$

for ( $\mathrm{t}$ in $\mathrm{tmin}: \operatorname{sim} \mathrm{T})\{$

\# Queue length at time (t-1) and maximum discharge flow rate at time (t)

QN4_1<- IO\$QN4[which(time==t-1)]

QL4_1 <- IO\$QL4[which(time==t-1)] / Kj

Voutmax4 <-IO\$Voutmax4[which(time==t)]

QNdp_1 <- IO\$QNdp[which(time==t-1)]

QLdp_1 <- IO\$QLdp[which(time==t-1)] / Kj

Voutmaxdp <-IO\$Voutmaxdp[which(time==t)]

QN3_1 <- IO\$QN3[which(time==t-1)]

QL3_1 <- IO\$QL3[which(time==t-1)] / Kj

Voutmax3 <-IO\$Voutmax3[which(time==t)]

QN2_1 <- IO\$QN2[which(time==t-1)]

QL2_1 $<-1 O \$ Q L 2[$ which(time $==\mathrm{t}-1)] / \mathrm{Kj}$

Voutmax2 <-IO\$Voutmax2[which(time==t)]

Vin2 <- IO\$Vin2[which(time==t)]

\# Estimate flows and queue if there is no queue spillback (base case)

\# at entry intersection, upstream intersection, and double-parking location

if $(a+b==0)\{$

Vout2 <- $\min (($ Vin2 + QN2_1), Voutmax2)

QN2 <- $\max \left(\left(\operatorname{Vin} 2+\mathrm{QN2} \_1-\right.\right.$ Vout2),0) \# "0" to prevent negative queue length

Vin3 $<-$ Vout2

Vout3 $<-\min (($ Vin3 + QN3_1), Voutmax3)

QN3 <- max ((Vin3 + QN3_1 - Vout3),0) \# "0" to prevent negative queue length

Vindp $<-$ Vout3

Voutdp $<-\min (($ Vindp + QNdp_1), Voutmaxdp)

QNdp <- max ((Vindp + QNdp_1 - Voutdp),0) \# "0" to prevent negative queue length

if $(\mathrm{QN} 3 / \mathrm{Kj}>=(\mathrm{D} 3-\mathrm{D} 2)) \mathrm{a}<-2$

if $(\mathrm{QNdp} / \mathrm{Kj}>=(\mathrm{Ddp}-\mathrm{D} 3)) \mathrm{b}<-1$

\}

\# Estimate flows and queue if queue spill over both entry and upstream intersections

\# at entry intersection, upstream intersection, and double-parking location

if $(a+b==3)\{$

Vout2 $<-0+$ divert * Voutmax 2

\# main difference from the base case

QN2 <- $\max ((\operatorname{Vin} 2+$ QN2_1 - Vout2),0) \# main difference from the base case

Vin3 $<-0 \quad$ \# main difference from the base case

Vout3 <- min(QN3_1, Voutmax3, Voutmaxdp) \# main difference from the base case

QN3 <- max ((QN3_1 - Vout3),0)

\# main difference from the base case

Vindp $<-$ Vout3

Voutdp $<-\min (($ Vindp + QNdp_1), Voutmaxdp)

QNdp $<-\max (($ Vindp + QNdp_1 - Voutdp $), 0)$

if $(\mathrm{QN} 3 / \mathrm{Kj}<(\mathrm{D} 3-\mathrm{D} 2)) \mathrm{a}<-0$ 


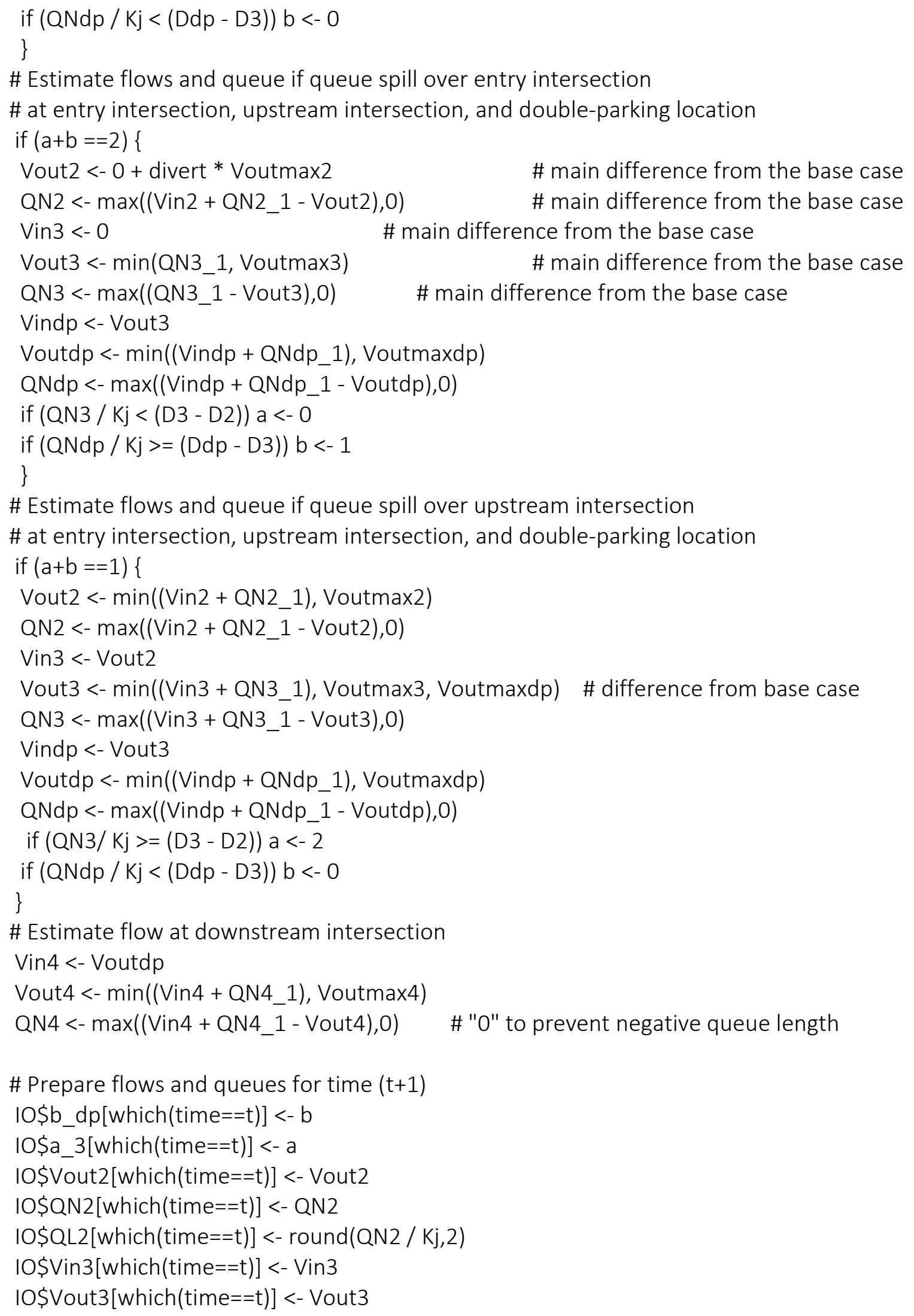




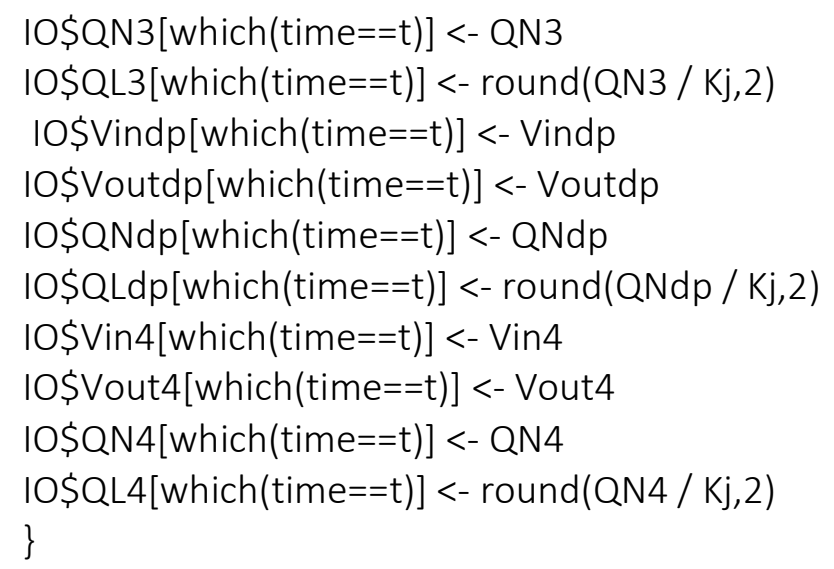




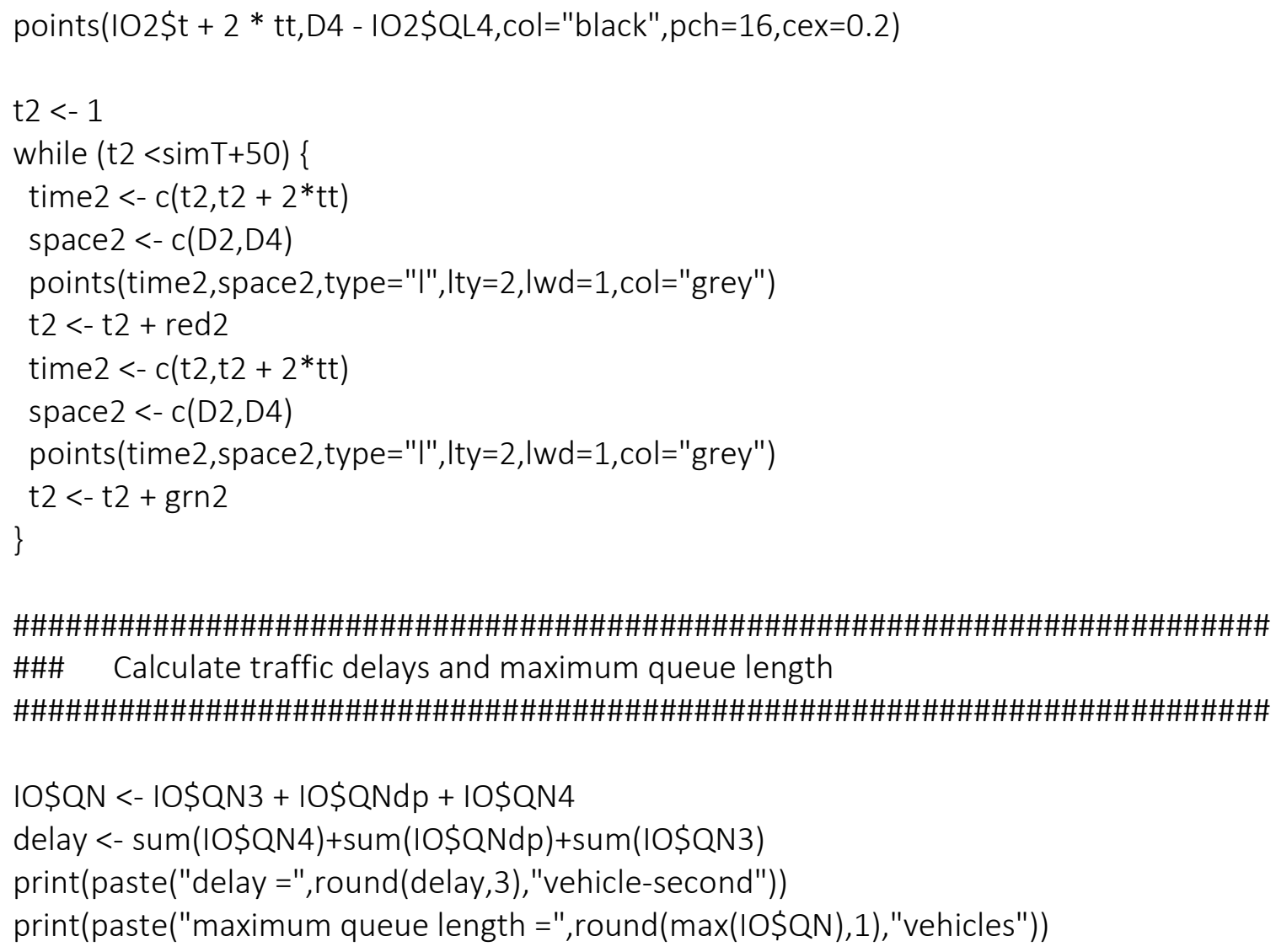

